\title{
A Mitotic Actin Regulating Pathway Induces Chromosomal Instability In Human Cancer Cells
}

\author{
Dissertation \\ for the award of the degree \\ "Doctor rerum naturalium" \\ of the Georg-August-Universität Göttingen \\ within the doctoral program "Molecular Biology of Cells" \\ of the Georg-August University School of Science (GAUSS)
}

submitted by

Elina Glaubke

from Osnabrück, Germany

Göttingen, February 2020 


\section{Thesis Committee}

Prof. Dr. Lutz Walter

Department of Primate Genetics

German Primate Center, Göttingen

Prof. Dr. Matthias Dobbelstein

Institute for Molecular Oncology

University Medical Center Göttingen

Dr. Julia Groß

Institute for Developmental Biochemistry

Section of Extracellular Signal Transduction

University Medical Center Göttingen

Dr. Melina Schuh

Department of Meiosis

Max Planck Institute for Biophysical Chemistry, Göttingen

Members of the Examination Board

Referee: $\quad$ Prof. Dr. Lutz Walter

Department of Primate Genetics

German Primate Center, Göttingen

$2^{\text {nd }}$ Referee: Prof. Dr. Matthias Dobbelstein

Institute for Molecular Oncology

University Medical Center Göttingen

\section{Further Members of the Examination Board}

Dr. Julia Groß

Institute for Developmental Biochemistry

Section of Extracellular Signal Transduction

University Medical Center Göttingen

Dr. Melina Schuh

Department of Meiosis

Max Planck Institute for Biophysical Chemistry, Göttingen

Prof. Dr. Andreas Janshoff

Institute for Biophysical Chemistry

University of Göttingen

Prof. Dr. Michael Meinecke

Institute for Cellular Biochemistry

University Medical Center Göttingen

Date of oral examination: 28.04 .2020 
The experiments were conducted at the Institute for Molecular Oncology, University Medical Center Göttingen, under the supervision of Prof. Dr. Holger Bastians. 


\section{Affidavit}

Hereby I declare that my doctoral thesis entitled "A Mitotic Actin Regulating Pathway Induces Chromosomal Instability In Human Cancer Cells" has been written independently with no other sources and aids than quoted.

Göttingen, February 2020

\section{Elina Glaubke}




\section{Table of Contents}

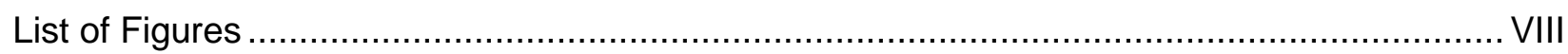

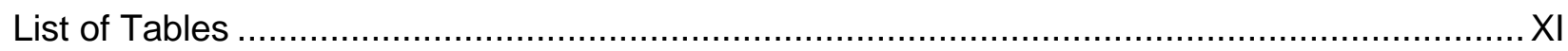

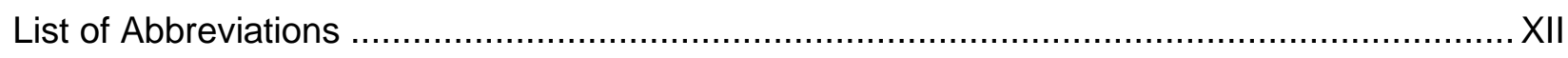

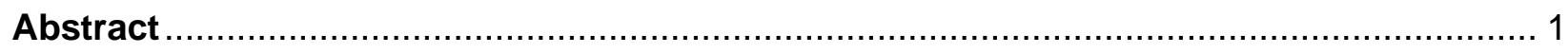

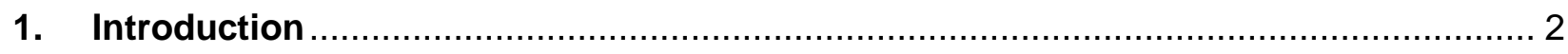

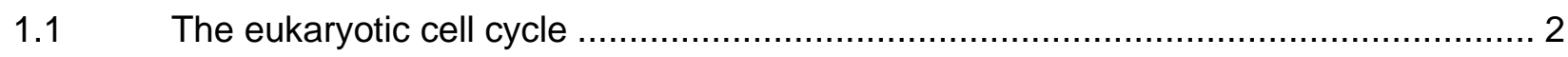

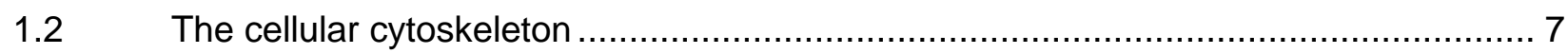

1.2.1 Assembly and regulation of the microtubule cytoskeleton ....................................... 7

1.2.2 Assembly and regulation of the actin cytoskeleton ……...................................... 9

1.2.3 Function and regulation of the cellular cytoskeleton in cell migration ........................ 14

1.2.4 Structure and function of the actin cytoskeleton in mitosis ................................... 20

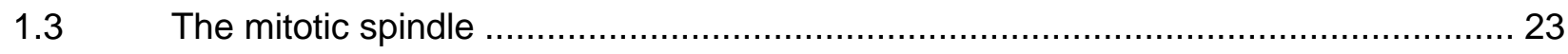

1.3.1 Chromosome dependent and independent assembly of the mitotic spindle ............... 23

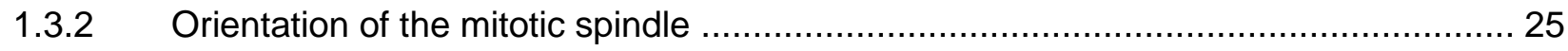

1.3.3 Function of the mitotic spindle in chromosome alignment and segregation................ 26

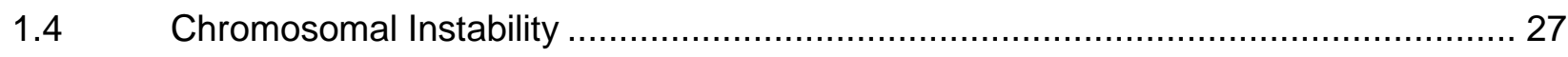

1.4.1 Mechanisms of whole chromosome instability …................................................. 28

1.4.2 Mechanisms of structural chromosome instability ................................................ 30

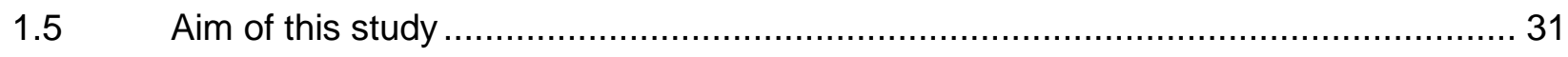

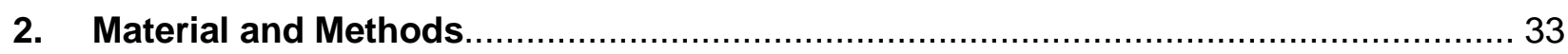

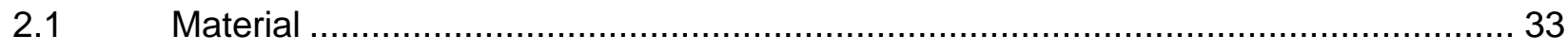

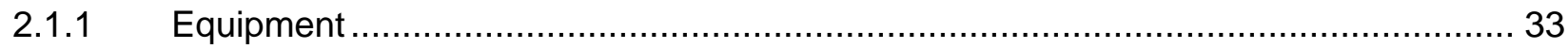




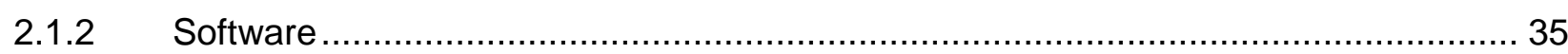

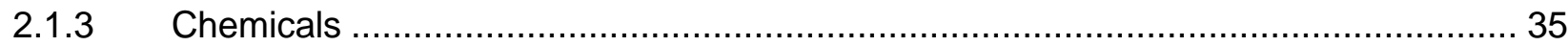

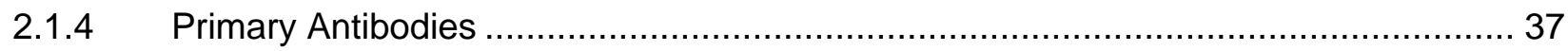

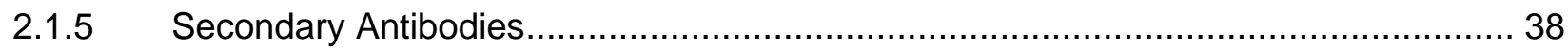

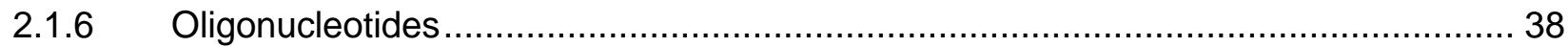

2.1.7 Plasmids

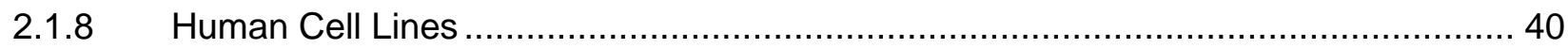

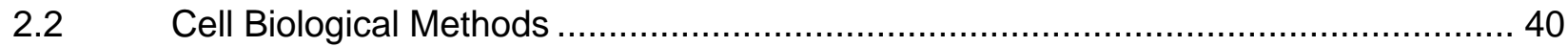

2.2.1 Cultivation of Human Cell Lines....................................................................... 40

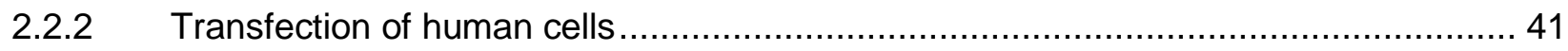

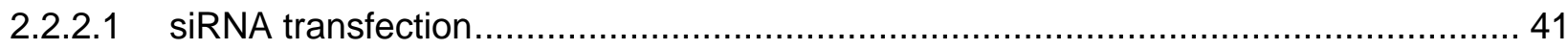

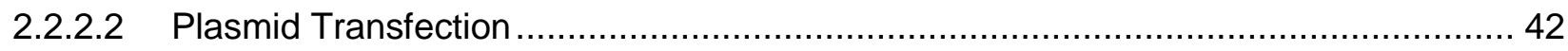

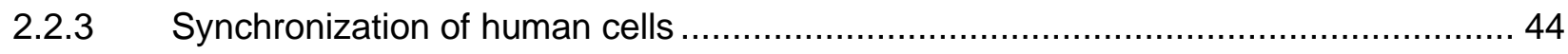

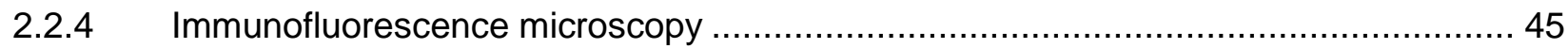

2.2.5 Analysis of spindle axis alignment of prometaphase cells .................................... 45

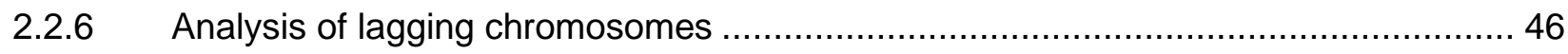

2.2.7 Analysis of microtubule plus-end assembly rates ............................................ 47

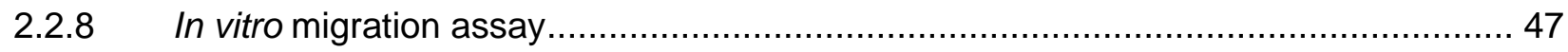

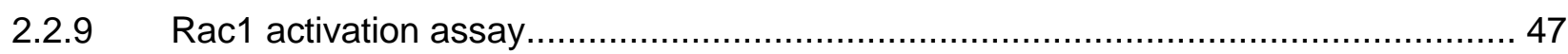

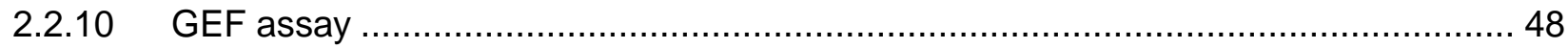

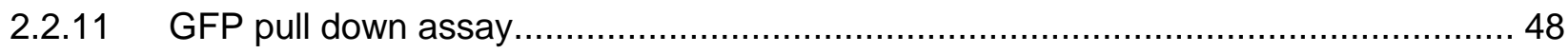

2.2.12 Detection and quantification of actin cytoskeleton intensities................................ 49

2.2.13 Analysis of F-actin structures using STED microscopy …..................................... 50

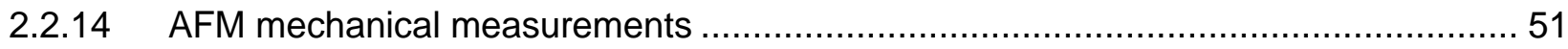


2.2.15 Actin cortex thickness measurements

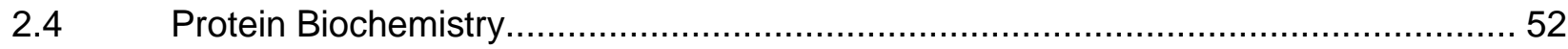

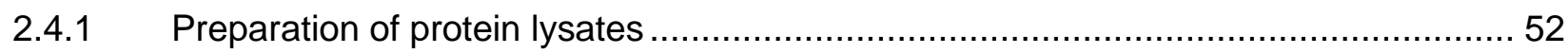

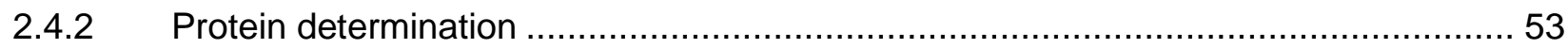

2.4.3 Sodium dodecyl sulfate polyacrylamide gel electrophoresis (SDS-PAGE) .............. 53

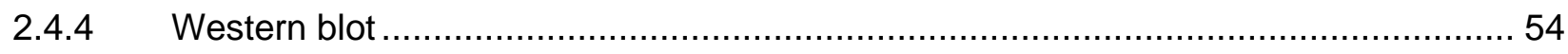

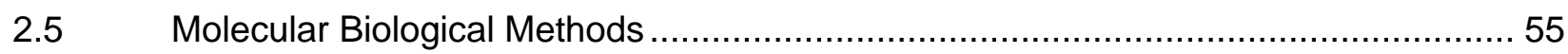

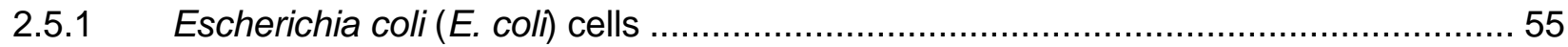

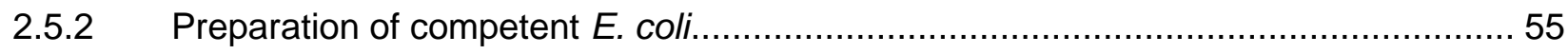

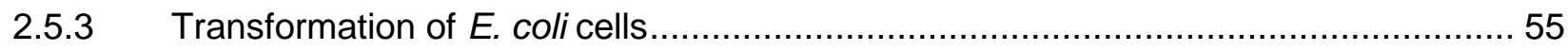

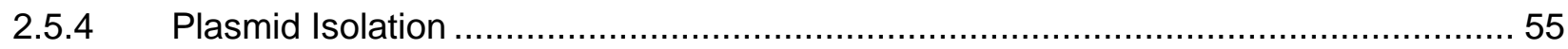

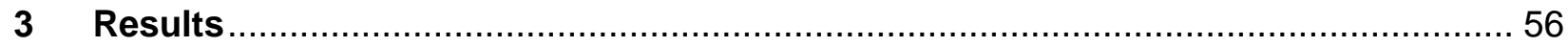

3.1 TRIO binds to EB1 at microtubule plus ends in a microtubule dynamic dependent

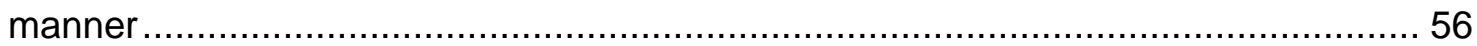

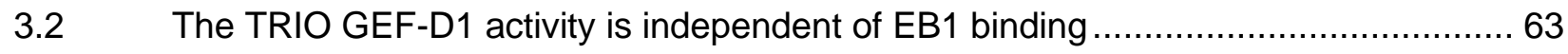

3.3 An increase in Rac1 activity requires EB1 binding of TRIO in mitotic cells............... 65

3.4 TRIO overexpression increases cell migration to a higher extend when EB1 binding is

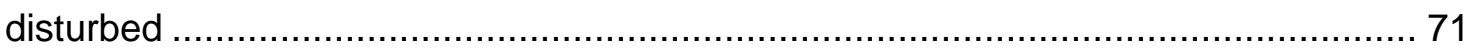

3.5 Overexpression of TRIO induces spindle axis misalignment, chromosome missegregation and chromosomal instability in an EB1 binding dependent manner....... 73

3.6 Chromosomal instability induced by overexpression of TRIO is rescuable by inhibition

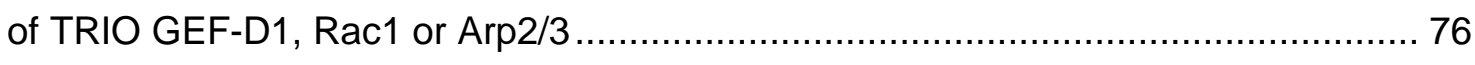

3.7 Increased mitotic Rac1 activity induces spindle axis misalignment, chromosome mis-

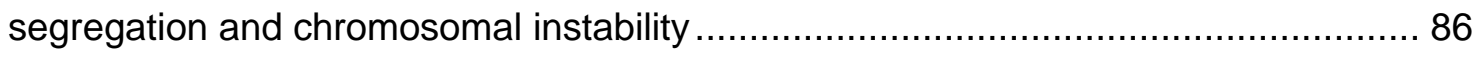

3.8 The mitotic F-actin cytoskeleton is regulated by a pathway containing microtubule plus-

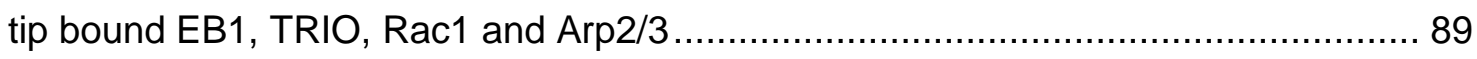




\section{Table of Contents}

3.9 Chromosomally unstable cells exhibit decreased mitotic actin cortex tension that is rescuable by inhibition of a microtubule dynamic, EB1, TRIO, Rac1, Arp2/3 dependent pathway 100

4 Discussion 106

4.1 Why is the TRIO-EB1 interaction affected by a Taxol dependent decrease, but not by a Nocodazole independent decrease of microtubule plus-end assembly rates? ......... 108

4.2 The role of EB1 binding for the functions of TRIO and its effector Rac1 .............. 109

4.3 The role of TRIO in the induction of whole and structural chromosomal instability ... 113

4.4 The EB1-TRIO-Rac1-Arp2/3 dependent pathway represents the missing link between microtubule dynamics and spindle positioning by modulating mitotic cortex tension 115

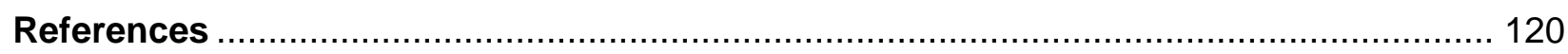

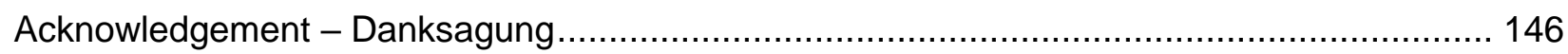

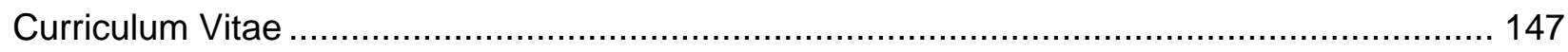




\section{List of Figures}

Figure 1.1: The phases of a eukaryotic cell cycle..................................................... 3

Figure 1.2: Cyclin-Cdk complexes are regulators of the cell cycle.................................... 5

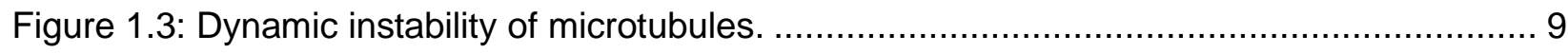

Figure 1.4: Actin filament assembly, nucleation and elongation factors............................ 12

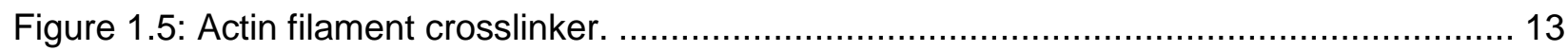

Figure 1.6: Regulation of actin cytoskeleton dynamics by Rho GTPases and their effectors.... 15

Figure 1.7: Regulation of Rho GTPases by GEFs, GAPs and GDIs.............................. 16

Figure 1.8: The multidomain protein TRIO and its functional domains. .............................. 18

Figure 1.9: Involvement of GEFs and their Rho GTPases in cell migration and proliferation..... 20

Figure 1.10: The role of the actin and microtubule cytoskeleton in mitotic spindle positioning... 22

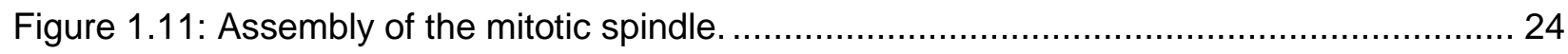

Figure 1.12: Modes of kinetochore-microtubule attachments. ........................................ 29

Figure 2.1: Schematic illustration for the calculation of the spindle axis angle..................... 46

Figure 3.1: TRIO co-localizes with EB1 at microtubules in an EB1 dependent manner............58

Figure 3.2: Interaction between TRIO and EB1 can be diminished by a decrease in microtubule plus-end assembly rates in chromosomally unstable HEK293T cells.

Figure 3.3: Generation of HCT116 cell clones stably expressing GFP, TRIO-WT-GFP or TRIOSRNN-GFP.

Figure 3.4: EB1-TRIO binding is not affected by a Nocodazole-induced increase of microtubule

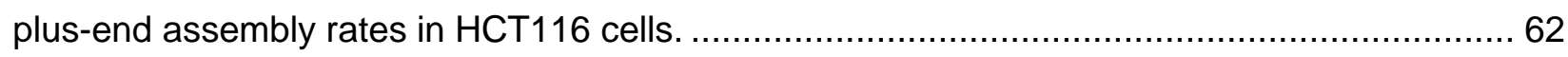

Figure 3.5: The GEF-D1 activity of TRIO is independent of EB1 binding........................... 64

Figure 3.6: Rac1 activity is reduced by TRIO inhibition, but not by a Taxol-induced decrease of microtubule plus-end assembly rates. 
Figure 3.7: EB1 binding of TRIO is needed for a stronger increase in Rac1 activity of mitotic cells. 69

Figure 3.8: EB1-TRIO binding is crucial for activating Rac1 in mitotic cells with moderate, longterm TRIO overexpression.

Figure 3.9: Overexpressed TRIO induces increased cell migration to a greater extend with EB1 binding deficiency.

Figure 3.10: TRIO overexpression leads to an EB1 binding dependent increase in spindle axis misalignment, in the amount of lagging chromosomes and in karyotype variability. 75

Figure 3.11: The increase in lagging chromosome formation in TRIO overexpressing HCT116 cells can be rescued by low dose Taxol as well as inhibition of TRIO GEF-D1, Rac1 or Arp2/3....... 79

Figure 3.12: TRIO overexpression causes an increase in anaphase bridges and DNA fragments that is only partially rescuable by low dose Taxol or inhibition of TRIO GEF-D1, Rac1 or Arp2/3.

Figure 3.13: TRIO overexpression induced karyotype variability in HCT116 cells is rescuable by low dose Taxol or by inhibition of TRIO GEF-D1, Rac1 or Arp2/3.

Figure 3.14: Overexpressed TRIO induces the formation of dicentric chromosomes and double minutes that are only partially or not rescuable by low dose Taxol or inhibition of Rac1 or Arp2/3.

Figure 3.15: Expression of constitutively active Rac1 leads to a slight increase in spindle axis misalignment and chromosome mis-segregation.

Figure 3.16: Expression of constitutively active Rac1 not only induces the generation of multinucleated cells, but also chromosomal instability..... 88

Figure 3.17: Mitotic actin cloud structures are regulated by a fraction of Arp2/3 that does not seem to be involved in the EB1, TRIO, Rac1, Arp2/3 dependent pathway.

Figure 3.18: The mitotic F-actin cytoskeleton is regulated by a microtubule dynamic dependent pathway in chromosomally unstable cells. 93

Figure 3.19: Knock down of CKAP5, EB1 or TRIO decreases the F-actin signal intensity in chromosomally unstable cells. 
Figure 3.20: Knock down of Rac1 or Arp3 leads to a decrease in the F-actin signal intensity of chromosomally unstable and chromosomally stable cells.

Figure 3.21: The F-actin signal intensity of chromosomally stable HCT116 cells is not affected by solely increasing microtubule plus-end assembly rates or by overexpression of constitutively active Rac1. 96

Figure 3.22: The F-actin signal intensity of chromosomally unstable SW620 cells is not affected by mild activation of RhoA or mild inhibition of formins, but by inhibition of RhoA or myosin II. . 99

Figure 3.23: Chromosomally unstable SW620 cells exhibit decreased mitotic cortex tension $\mathrm{T}_{0}$ that is rescuable by inhibition of the microtubule dynamic, EB1, TRIO, Rac1, Arp2/3 dependent pathway, but not by myosin II inhibition. 101

Figure 3.24: The actin cortex thickness of is not affected by downregulation of components of the microtubule plus-end-EB1-TRIO-Rac1-Arp2/3 pathway. 103

Figure 3.25: STED microscopy visualizes changes in the F-actin cytoskeleton of mitotic SW620 cells after inhibition of Rac1 and Arp2/3, but not after Taxol-induced restoration of microtubule plus-end assembly rates. 105

Figure 4.1: Model for the microtubule plus-end-EB1-TRIO-Rac1-Arp2/3 dependent pathway and its role in the development of CIN via decreasing actin cortex tension. 107

Figure 4.2: Correlations between the induction of W-CIN and S-CIN. 114

Figure 4.3: Model for the impact of actin cortex architecture and myosin II on cortical tension. 117 


\section{List of Tables}

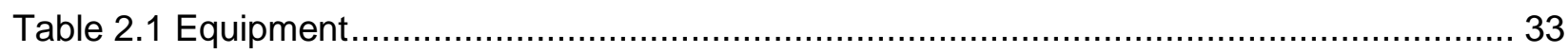

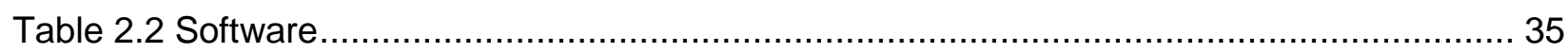

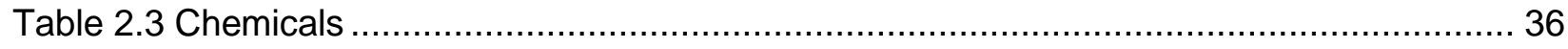

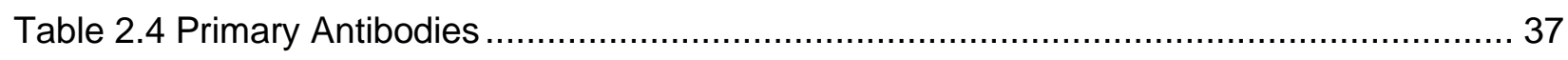

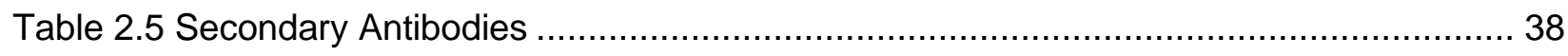

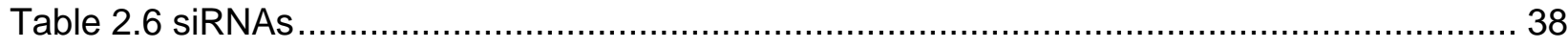

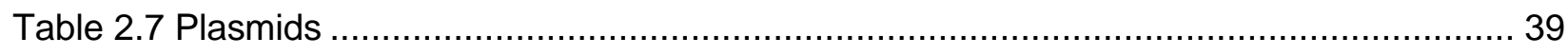

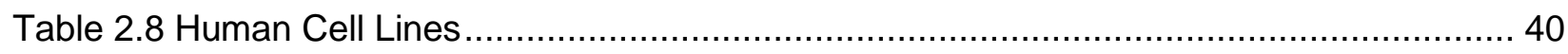

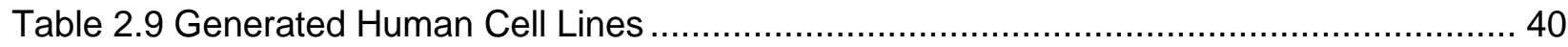




\section{List of Abbreviations}

+TIP microtubule plus-end binding protein

ABP actin-binding protein

AFM atomic force microscopy

APC/C anaphase-promoting complex/cyclosome

Arp2/3 actin-related protein 2/3

ATM ataxia telangiectasia mutated

ATR ATM-Rad3-related

$\mathrm{B} / \mathrm{F} / \mathrm{B}$ cycles breakage/fusion/bridge cycles

BER base excision repair

BSA bovine serum albumin

CAK Cdk-activating kinase

Cdk cyclin-dependent kinase

ch-TOG colonic and hepatic tumor overexpressed gene

CIN chromosome instability

CKI Cdk inhibitor proteins

CLIP170 cytoplasmic linker protein 170

Cobl cordon bleu

$\mathrm{CP} \quad$ capping protein

$\mathrm{CPC} \quad$ chromosomal passenger complex

CRC colorectal cancer cells

DAAM disheveled-associated activator of morphogenesis

DAD diaphanous autoregulatory domain

DDR DNA damage response

$\mathrm{DH}$ domain Dbl homology domain

DHR1 DOCK homology region 1

DHR2 DOCK homology region 2

Dia diaphanous

DID diaphanous inhibitory domain

DME dimethylenastrone

DMSO dimethylsufoxide

dsDNA double-stranded DNA

EB end binding protein

EB1 end-binding protein 1 


\begin{tabular}{ll}
\hline ECM & extracellular matrix \\
EDTA & ethylenediaminetetraacetic acid \\
ERM & ezrin-radixin-moesin \\
F-actin & filamentous actin \\
FCS & fetal calf serum \\
FH1 & formin homology 1 \\
FH2 & formin homology 2 \\
FHOD & fomin homology domain-containing protein \\
FMN & formin \\
FRL & formin-related gene in leukocytes \\
G-actin & globular actin \\
GAP & GTPase-activating protein \\
GBD & Rho GTPase binding domain \\
GDI & guanine nucleotide dissociation inhibitor \\
GEF & guanine nucleotide exchange factor \\
GFP & green fluorescent protein \\
HR & homologous recombination \\
INF & inverted formin \\
JMY & junction mediating regulatory protein \\
JNK & Jun N-terminal kinase \\
LB medium & Luria Bertani medium \\
LGN & Leu-Gly-Asn repeat enriched protein \\
LIMK & LIM kinase \\
Lmod & leiomodin \\
MAP & microtubule-associated protein \\
MAPK & mitogen-activated protein kinase \\
MCAK & mitotic centromere-associated kinesin \\
MCC & mitotic checkpoint complex \\
MIN & microsatellite instability \\
MLC & myosin light chain \\
MMEJ & microhomology-mediated end joining \\
MMR & mismatch repair \\
MTOC & microtubule organization center \\
Nav1 & Navigator 1 \\
NEBD & nuclear envelope breakdown \\
& \\
\hline
\end{tabular}




$\begin{array}{ll}\text { NER } & \text { nucleotide excision repair } \\ \text { NHEJ } & \text { nonhomologous end joining } \\ \text { NPF } & \text { nucleation promoting factor } \\ \text { NTR } & \text { nuclear transport receptor } \\ \text { NuMA } & \text { nuclear mitotic apparatus protein } \\ \text { N-WASP } & \text { neuronal WASP } \\ \text { PAK } & \text { p21-activated kinase } \\ \text { PBS } & \text { phosphate buffered saline } \\ \text { PCM } & \text { pericentriolar material } \\ \text { PH domain } & \text { pleckstrin homology domain } \\ \text { Rac1 } & \text { Ras-related C3 botulinum toxin substrate 1 } \\ \text { RB protein } & \text { retinoblastoma protein } \\ \text { Rho } & \text { Ras homolog } \\ \text { ROCK } & \text { Rho-associated kinase } \\ \text { RT } & \text { room temperature } \\ \text { SAC } & \text { spindle assembly checkpoint } \\ \text { SCAR } & \text { suppressor of cAR } \\ \text { S-CIN } & \text { structural chromosome instability } \\ \text { SDS-PAGE } & \text { Sodium dodecyl sulfate polyacrylamide gel electrophoresis } \\ \text { SEM } & \text { scanning electron microscopy } \\ \text { SMLM } & \text { single molecule localization based super-resolution microscopy } \\ \text { SSDNA } & \text { single-stranded DNA } \\ \text { STED } & \text { stimulated emission depletion } \\ \text { STEF } & \text { Sif and TIAM1-like exchange factor } \\ \text { Tara } & \text { TRIO-associated repeat on actin } \\ \text { TIAM1 } & \text { T-lymphoma invasion and metastasis inducing protein 1 } \\ \text { TRIO } & \text { triple functional domain protein } \\ \text { TRIS } & \text { tris(hydroxymethyl)aminomethane } \\ \text { VCA domain } & \text { verprolin homology, central and acidic sequence } \\ \text { WASH } & \text { WASP and SCAR homolog } \\ \text { WASP } & \text { Wiskott-Aldrich Syndrome protein } \\ \text { WAVE1-3 } & \text { WASP and verprolin homologs 1-3 } \\ \text { W-CIN } & \text { whole chromosome instability } \\ \text { WHAMM } & \text { WASP homolog associated with actin, membranes and microtubules } \\ \text { Y-TuRC } & \text { V-tubulin ring complex } \\ & \end{array}$




\section{Abstract}

Chromosome instability (CIN) is a hallmark of human cancer and causes structural (S-CIN) as well as whole (W-CIN) chromosome aberrations. Cancers exhibiting the CIN phenotype are characterized by a large genetic heterogeneity that is associated with an increased adaptability of cancer cells and with a poor patient's prognosis. In order to improve cancer therapy, it is of particular interest to unravel the mechanisms underlying CIN. Recently, enhanced mitotic microtubule growth rates were identified to contribute to CIN via inducing transient mitotic spindle mis-orientation, thereby triggering an increase in chromosome mis-segregation. Moreover, a pathway containing the microtubule plus-end binding protein EB1, the guanine exchange factor TRIO and its Rho GTPase Rac1, as well as the F-actin regulating Arp2/3 complex was identified to be involved in generation of the spindle orientation defect in cells with increased microtubule plus-end growth rates. However, many links within this pathway were still elusive and their investigation was the main aim of this study.

This study revealed that EB1 and TRIO interact at microtubule plus-ends and that this interaction depends on microtubule dynamics. Moreover, it could be shown that this interaction is required for downstream activation of Rac1 and Arp2/3 in mitotic cells, which in turn results in reorganization of the actin cytoskeleton. Upon hyperactivation of this pathway, such as through increased microtubule plus-end growth rates found in CIN cells, the connectivity and thus density of the actin cortex increases, thereby causing a decreased cortical tension. Since a reduced cortical tension is associated with defects in spindle orientation, this triggers chromosome missegregation and thus CIN. Interestingly, an upregulation of TRIO did not only induce whole chromosome instability in an EB1 binding dependent manner via the introduced pathway, but also structural chromosome instability. S-CIN did not only arise through the EB1 binding dependent pathway but also through an EB1 binding independent hitherto unknown pathway. Moreover, TRIO upregulation also resulted in enhanced cell migration in an EB1 binding independent manner. Therefore, these findings do not only give rise to further details and links of a CIN-inducing pathway connecting increased microtubule plus-end growth rates and spindle mis-orientation, but also further confirm and expand the role of TRIO in the development of genetic heterogeneity and metastasis. 


\section{Introduction}

\subsection{The eukaryotic cell cycle}

Common to every living creature is their reproduction by a cycle of duplication and division, the so-called cell cycle. While this cycle results in the generation of a completely new organism for unicellular species, multiple cycles following a complex sequence are required for the development of multicellular species (Alberts et al., 2015). Moreover, cell duplication and division are continuously ongoing even in the adult multicellular organism to ensure replacement of dying cells. This process needs to be tightly regulated, as defects in the regulation can induce uncontrolled cell division, a common feature of all cancer cells (Clurman \& Roberts, 1995; Collins et al., 1997; Hanahan \& Weinberg, 2000). The fundamental process underlying the cell cycle is the duplication of the cell's genetic information and its equal distribution into two daughter cells. These events take place at different phases of the cell cycle: duplication of chromosomes occurs in S phase via DNA synthesis, while segregation of chromosomes and cell division takes place during mitosis (Murray \& Hunt, 1993). These phases are separated by two gap phases, $\mathrm{G}_{1}$ between mitosis and $S$ phase and $G_{2}$ between $S$ phase and mitosis, that allow time for growth and doubling of the cell's organelles and protein mass. $G_{1}, S$ and $G_{2}$ phases together are summarized as interphase. Subsequently to mitosis, $G_{1}$ phase begins, allowing not only time for the cell to grow but also to take notice of the environmental conditions and whether they are suitable for another round of duplication and division. In case of unfavorable extracellular conditions, $G_{1}$ phase is extended or the cell even withdraws temporarily or permanently from the active cell cycle by entering the resting phase $G_{0}$. However, in the presence of proliferative stimuli that indicate favorable environmental conditions, cells pass the so-called restriction point at the end of $G_{1}$ phase, thereby entering $S$ phase in an irreversible manner. During $S$ phase, cells do not only replicate their DNA, but also perform centrosome duplication (Laskey et al., 1989). Moreover, the resulting sister chromatids are paired and held together by the cohesin protein complex (Skibbens, 2009). After $S$ phase, cells enter $G_{2}$ phase, characterized by synthesis and activation of proteins that are required for subsequent mitosis. $G_{2}$ phase also allows time for the repair of DNA doublestrand breaks that arise during DNA replication (Cuddihy \& O'Connell, 2003). Following $G_{2}$ phase, cells enter mitosis that consists of prophase, prometaphase, metaphase, anaphase and telophase (Murray \& Hunt, 1993) (Figure 1.1). In prophase, chromosome condensation occurs. Additionally, the two centrosomes separate and start to migrate along the nuclear envelope in opposite directions (Tanenbaum \& Medema, 2010). During this process, microtubule nucleation at centrosomes increases, which results in the formation of a bipolar mitotic spindle. In the following 
prometaphase, nuclear envelope breakdown (NEBD) takes place and kinetochores are assembled at the centromeric region of the duplicated chromatids, thereby providing a platform that links the chromosomes to microtubules emanating from the mitotic spindle (Cheeseman \& Desai, 2008). The process, in which microtubules begin to search for and attach to chromosomal kinetochores, takes place in late prometaphase and ends in metaphase with all sister chromatids being attached to microtubules from opposing poles of the mitotic spindle. Upon alignment of all chromosomes at the spindle equator forming the metaphase plate, a cohesin subunit is cleaved by the protease separase that has been activated by the anaphase-promoting complex/cyclosome $(\mathrm{APC} / \mathrm{C})$. This results in disassembly of the cohesin protein complex and consequently in the separation of sister chromatids (Nakajima et al., 2007). In anaphase A, sister chromatids are pulled to opposite spindle poles by kinetochore microtubule depolymerization, whereas in anaphase $B$ the two spindle poles themselves move further apart by polar microtubules pushing against each other (Goshima \& Scholey, 2010; Inoué \& Ritter, 1975). During this process, the cell

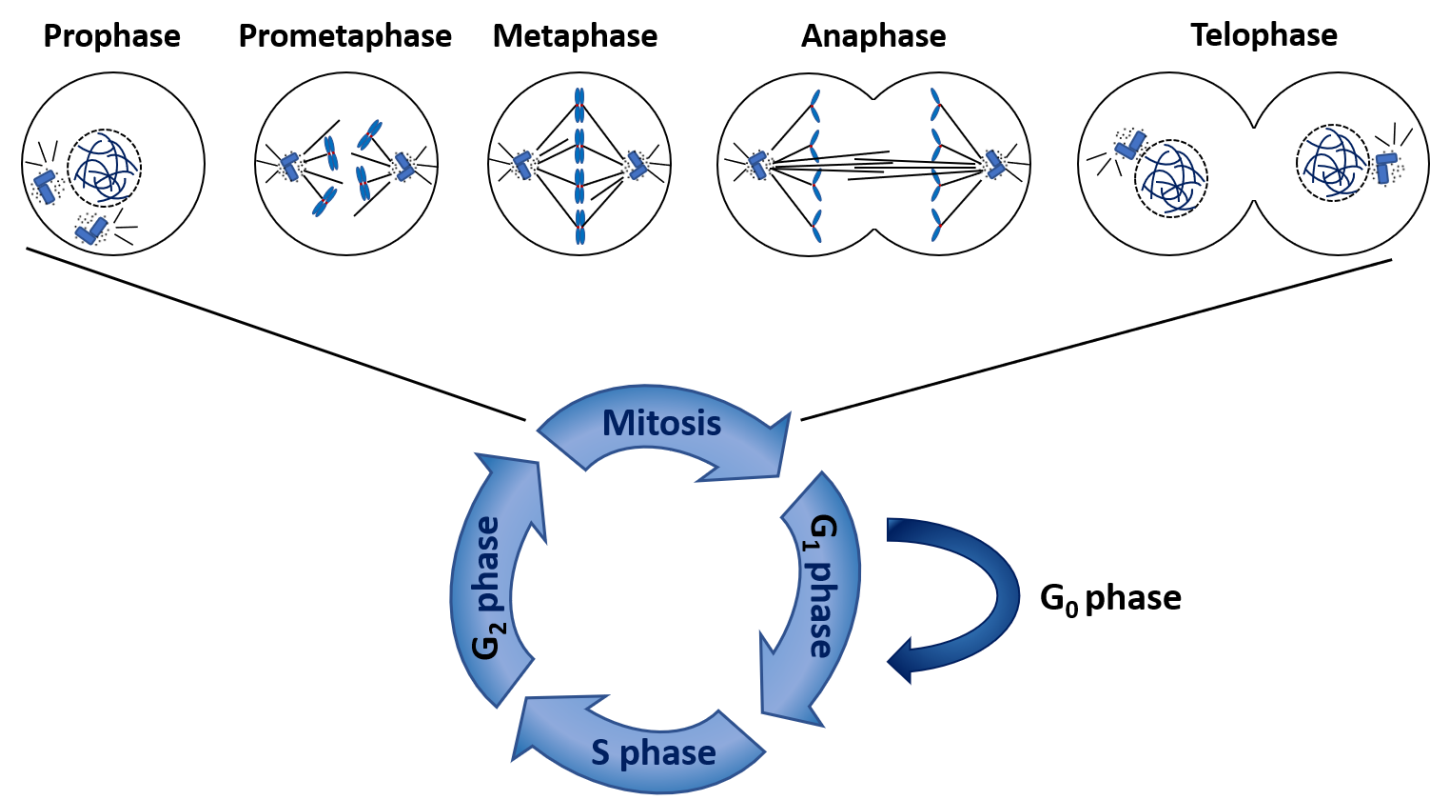

Figure 1.1: The phases of a eukaryotic cell cycle. The eukaryotic cell cycle consists of interphase containing the three phases $\mathrm{G}_{1}$ (first gap phase) $\mathrm{S}$ (DNA synthesis), and $\mathrm{G}_{2}$ (second gap phase) and mitosis. $\mathrm{G}_{0}$ phase is a resting phase entered by cells that withdraw from the active cell cycle. The five sub-phases of mitosis are prophase, prometaphase, metaphase, anaphase and telophase. In prophase, chromosome condensation takes place and formation of a bipolar spindle is initiated. Breakdown of the nuclear envelope is accomplished in prometaphase, as well as kinetochore assembly, followed by chromosome capturing by mitotic spindle derived microtubules. After complete chromosome alignment at the metaphase plate, sister chromatids become separated and pulled to opposite spindle poles during anaphase. Finally, new nuclear envelopes are built around the separated, decondensing chromosome masses and a cleavage furrow is formed in telophase, followed by complete cytokinesis. Modified from Walczak et al. (2010). 
elongates and an actomyosin ring assembles at the division plane. In telophase, separate nuclear envelops form around the segregated chromosomes that begin to decondense again and contraction of the actomyosin ring leads to the formation of a cleavage furrow (Glotzer, 2005). Finally, cytokinesis ends up with the complete separation of the dividing cell in two daughter cells.

To ensure an error-free cell cycle progression, control systems govern major cell cycle events, such as entry into $S$ phase, $G_{2} / M$ transition or metaphase-to-anaphase transition. Control of many cell cycle events is mediated by the oscillating activation and inactivation of members of a family of cyclin-dependent kinases (Cdks) (Arellano \& Moreno, 1997). Cyclical activity of these Cdks is not archived by the protein level of Cdks, as this is constant throughout the cell cycle, but by binding of so-called cyclins that activate the protein kinase. In contrast to Cdk protein levels, cyclin levels change cyclically, thereby allowing for activation of Cdks at specific stages of the cell cycle (Figure 1.2). Depending on the cyclin-Cdk complex and its activity, cell cycle stage specific events are triggered by modulation of transcriptional, translational and DNA replication-related processes as well as of mitotic checkpoints. D cyclins bind to CDK4 and CDK6 during $G_{1}$ phase resulting in the phosphorylation of retinoblastoma $(\mathrm{Rb})$ protein (Harbour \& Dean, 2000; Malumbres \& Barbacid, 2005). $\mathrm{Rb}$ in turn releases its binding partner and transcription factor E2F that triggers transcription of genes important for DNA replication. Further phosphorylation of Rb induced by cyclin $E / C D K 2$ complexes that form at late $G_{1}$ phase finally results in $S$ phase entry. During $S$ phase, cyclin A binds to CDK2, thereby replacing cyclin $E$. This complex initiates and regulates DNA replication by triggering the assembly of the pre-replication complex, but at the same time preventing the assembly of additional replication complexes. Cyclin A binds to CDK1 at the beginning of $G_{2}$ phase and supports the assembly and activation of cyclin B/CDK1 complexes at the end of $G_{2}$ phase. This complex phosphorylates several proteins that are involved in chromosome condensation, nuclear envelope breakdown and spindle formation, thereby triggering entry into mitosis (Nigg, 2001). Degradation of cyclin B takes place during mitosis and the resulting inactivation of $C D K 1$ promotes mitotic exit. As binding of cyclins only allows for partial activation, phosphorylation of Cdks by the Cdk-activating kinase (CAK) is required for full activation of the Cdk-cyclin complex (Lolli \& Johnson, 2005). In addition to the cyclically changing cyclin levels, further mechanisms are involved in the control of Cdk activity. This includes inactivation of cyclin-Cdk complexes through phosphorylation by the kinases Wee1 or Myt1, whereas removal of these phosphates by the phosphatase Cdc25 can restore the cyclin-Cdk activity (Den Haese et al., 1995; Malumbres \& Barbacid, 2005; D. O. Morgan, 1997). Inactivation of cyclin-Cdk levels is also archived by binding of Cdk inhibitor proteins (CKIs). Dysregulated expression and activity of cyclins and Cdks lead to uncontrolled cell proliferation and is frequently 
observed in cancer cells (Deshpande et al., 2005). In healthy cells, DNA damage induced by environmental factors or by errors in DNA replication results in cell cycle arrest or, in case of severe DNA damage, in apoptosis (Zhou \& Elledge, 2000). In order to stop the cell cycle from continuing until the DNA damage has been repaired, various protein kinases are recruited to the site of DNA damage. They trigger a signaling cascade ending with inhibition of the CDK1/cyclin $B$ complex and thereby preventing entry into mitosis. In response to DNA damage and replication stress, the replication fork is slowed or stalled to allow time for DNA damage repair (Rickman \& Smogorzewska, 2019). Upon activation of the DNA damage response (DDR) signaling, the kinase ATM (ataxia telangiectasia mutated) is primarily recruited to DNA double strand breaks, whereas the kinase ATR (ATM-Rad3-related) responds to various DNA damages, such as DNA single and double strand breaks (Maréchal \& Zou, 2013). Following ATM/ATR binding to the DNA damage site, ATM and ATR recruit the kinases Chk2 and Chk1, respectively (Jackson \& Bartek, 2009). These kinases phosphorylate cell cycle arrest-inducing target proteins, with p53 as major target. Activated p53 stimulates the transcription of diverse genes, including the gene coding for the cyclin-dependent kinase inhibitor p21. p21 in turn binds and inactivates cyclin E/CDK2 and

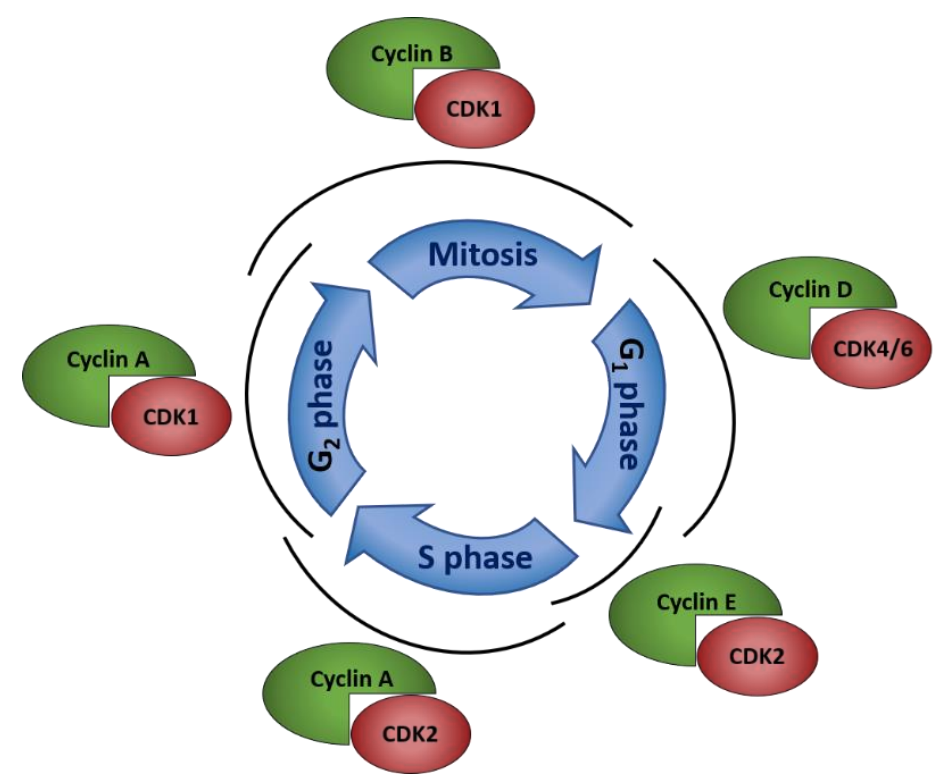

Figure 1.2: Cyclin-Cdk complexes are regulators of the cell cycle. Progression through the cell cycle is controlled by the presence of different cyclin-Cdk complexes at specific cell cycle stages: In $\mathrm{G}_{1}$ phase, Cyclin D binds to CDK4 and CDK6, while cyclin E/CDK2 complexes form at the end of $\mathrm{G}_{1}$ phase triggering expression of genes required for DNA replication. Upon entry into $S$ phase, cyclin A binds to CDK2 for initiation and regulation of DNA replication. At the beginning of $\mathrm{G}_{2}$ phase cyclin A binds to CDK1 and is replaced by cyclin $B$ at the end of $G_{2}$, thereby promoting entry into mitosis. Mitotic exit is induced by cyclin B degradation. Modified from Suryadinata et al. (2010). 
cyclin A/CDK2 complexes, thereby arresting the cell in $\mathrm{G}_{1}$. Additionally, Chk1 and Chk2 phosphorylate and thus inactivate members of the Cdc25 protein phosphatase family. These are no longer able to activate cyclin B/CDK1 complexes, thus blocking entry into mitosis. Upon cell cycle arrest, different cell cycle dependent DNA repair mechanisms have enough time to fulfil their task. The major DNA repair mechanisms include base excision repair (BER), nucleotide excision repair (NER), mismatch repair (MMR), homologous recombination (HR), nonhomologous end joining (NHEJ) and microhomology-mediated end joining (MMEJ) (Chatterjee \& Walker, 2017; Sallmyr \& Tomkinson, 2018).

While cyclin-Cdk complexes are responsible for the control of $G_{1} / S$ and $G_{2} / M$ transition, the spindle assembly checkpoint (SAC) controls metaphase-to-anaphase transition and ensures that this transition only takes place after all chromosomes have been properly attached to microtubules emanating from the mitotic spindle (Lara-Gonzalez et al., 2012). This prevents chromosome missegregation and thereby assures genomic stability. The major effector of the SAC is the mitotic checkpoint complex (MCC) that consists of the proteins MAD2, BUBR1, Bub3, Cdc20 and Mad2. The MCC assembles at unattached kinetochores and prevents Cdc20 from activating the APC/C, thereby avoiding premature separation of sister chromatids. In case of correct microtubulekinetochore attachment, the MCC disassembles from the kinetochore. The released Cdc20 activates the $A P C / C$, which in turn causes ubiquitination of cyclin $B$ and securin via its EB3 ubiquitin ligase activity. The subsequent proteasomal degradation of securin leads to release of separase that cleaves the cohesin subunit of the cohesin complex, thereby promoting separation of sister chromatids. APC/C-mediated degradation of cyclin B inactivates Cdk1 and thus induces mitotic exit. Another regulator of SAC activation is kinetochore tension (Maresca \& Salmon, 2010). Only when kinetochores from sister chromatids are properly attached to microtubules from opposing spindle poles, pulling forces of the mitotic spindle generate tension at the kinetochores that stabilizes the microtubule-kinetochore attachment. In contrast, weak tension due to incorrect attachments destabilizes binding of microtubules to kinetochores and allows reattachment to microtubules from the correct spindle pole. However, the SAC is not able to detect merotelic attachments, in which a single kinetochore is attached to microtubules emanating from both spindle poles, thereby inducing lagging chromosomes and chromosomal instability (Gregan et al., 2011). Correction of these merotelic attachments involves Aurora B kinase that is highly concentrated at the inner centromere where it destabilizes microtubules rather from the incorrect spindle pole due to spatial reasons (Cimini et al., 2006). 


\subsection{The cellular cytoskeleton}

Inside a cell many processes take place that require changes in the cell's shape and rearrangement of internal components including cell cycle dependent cell growth and division, but also processes such as adhesion and migration. To overcome these challenging tasks, the cell possesses of a dynamic filamentous system, the cytoskeleton. There are three major cytoskeletal filaments, namely actin filaments, microtubules and intermediate filaments (Fletcher \& Mullins, 2010; Hohmann \& Dehghani, 2019). Actin filaments determine the shape and tension of the cell's surface and are required for cell migration and cytokinesis. Microtubules act as platform for intracellular transport and are the major components of mitotic spindles, but also play a role for cell migration (Etienne-Manneville, 2013; Kaverina \& Straube, 2011). Intermediate filaments organize internal structures of the cell and are involved in forming the nuclear lamina and cell-cell junctions (Hohmann \& Dehghani, 2019). During mitosis, reorganization of the actin cytoskeleton is necessary to allow for correct spindle assembly and positioning and thereby proper chromosome segregation (Cadart et al., 2014). For this reason, not only microtubules forming the mitotic spindle are crucial for early mitotic processes but also a tightly regulated rearrangement of actin filaments. For a better understanding of the role of microtubules and actin filaments in mitosis, it is important to have a closer look at their assembly and regulation as well as their interplay and impact on the cell surface tension.

\subsubsection{Assembly and regulation of the microtubule cytoskeleton}

Microtubules are polymers of heterodimerized $\alpha$ - and $\beta$-tubulin subunits. These $\alpha \beta$-tubulin heterodimers are bound head to tail, thereby generating a protofilament (Alberts et al., 2015). The parallel assembly of 13 protofilaments leads to the formation of a hollow cylindrical structure, the microtubule. As major microtubule nucleator, $y$-tubulin forms together with other proteins the socalled $y$-tubulin ring complex ( $\gamma$-TuRC), which is usually found at the centrosomal microtubule organization center (MTOC) and serves as scaffold for initial microtubule assembly (Hohmann \& Dehghani, 2019). Strong interactions between longitudinal connected $\alpha \beta$-tubulin heterodimers and between lateral connected $\alpha-\alpha$ - or $\beta$ - $\beta$-monomers are responsible for the microtubule stiffness and allow microtubule turnover only at the ends (Alberts et al., 2015). As all $\alpha \beta$-tubulin heterodimers point in the same direction within the microtubule structure, they bear one end with a-tubulin exposed, called the minus end, and one end with $\beta$-tubulin exposed, called the plus end, which has been shown to grow and shrink more rapidly. Microtubule dynamics highly depend on the binding and hydrolysis of GTP bound to $\beta$-tubulin, as in a GTP bound state tubulin tends to polymerize, while it rather depolymerizes after hydrolysis of GTP to GDP. In case of microtubule 
growth, GTP bound $\beta$-tubulin polymerizes at microtubule ends, thereby forming a so-called GTP cap. However, when the GTP of $\beta$-tubulin at the GTP cap hydrolyzes faster to GDP than new GTP bound $\beta$-tubulin is added to the microtubule end, depolymerization of tubulin subunits occurs leading to microtubule shrinking. This process is called a catastrophe, whereas switching to microtubule growth again is called a rescue (Figure 1.3). In addition to the concentration of GTP bound $\beta$-tubulin within the cell, microtubule dynamics are also regulated by microtubuleassociated proteins (MAPs) (Bowne-Anderson et al., 2015). They can have a stabilizing or destabilizing effect, that several MAPs execute by their function as polymerase or depolymerase. While some MAPs execute their function along the length of the microtubule, other MAPs belong to a group of microtubule plus-end binding proteins (+TIP). One member of this + TIP family is the microtubule polymerase ch-TOG/XMAP215 (colonic and hepatic tumor overexpressed gene), which catalyzes the polymerization of tubulin dimers at the microtubule plus-end (Brouhard et al., 2008). The opposite effect is observed for the microtubule depolymerase MCAK (mitotic centromere-associated kinesin), that triggers dissociation of microtubule plus-end-located tubulin dimers (Hunter et al., 2003). Another important family of +TIPs are end binding proteins (EBs) (Hohmann \& Dehghani, 2019). EB proteins bind to growing microtubule plus-ends, where they recruit other factors, such as ch-TOG and MCAK, that affect microtubule dynamics or they act as a link between microtubule plus-ends and other cellular structures (Honnappa et al., 2009; Tirnauer \& Bierer, 2000). In this context, an EB1-mediated link between microtubule plus-ends and the cell cortex has been shown to be crucial for correct mitotic spindle assembly, dynamics and positioning as well as chromosome alignment and maintenance of chromosome stability (Rogers et al., 2002; Tirnauer \& Bierer, 2000). This observed link was reported to be generated by EB1 interacting with the guanine nucleotide exchange factor (GEF) RhoGEFD2 in Drosophila or with APC (adenomatous polyposis coli) in order to facilitate their delivery or interaction with specific sites at the cell cortex (Green et al., 2005; Rogers et al., 2002; Rogers et al., 2004).

Not only MAPs have been found to affect microtubule dynamics, but also chemical compounds, such as Taxol and Nocodazole. Interestingly, these two drugs cause different effects at different concentrations: At micromolar concentrations, Taxol stabilizes microtubules while Nocodazole causes microtubule depolymerization and both result in blocking mitosis (Lu et al., 2012; Yang \& Horwitz, 2017). At concentrations within a low to mediate nanomolar range, however, both drugs were shown to strongly inhibit microtubule dynamics, meaning both growth and shrinkage events (Jordan et al., 1992; Vasquez et al., 1997). This in turn results in aberrant mitosis and aneuploidy. Interestingly, Taxol was shown to suppress microtubule assembly preferentially at the plus ends (Derry et al., 1998). In chromosomally unstable cancer cells, which are known to exhibit increased 
microtubule plus end growth rates in contrast to chromosomally stable cells, even subnanomolar concentrations of Taxol were sufficient to decrease microtubule plus end growth rates (Berger, 2016; Ertych et al., 2014). Although Nocodazole also inhibits microtubule dynamics at low nanomolar concentrations (Vasquez et al., 1997), it has been shown to increase microtubule plus end growth rates in chromosomally stable cells when used at even lower nanomolar or at subnanomolar concentrations (Berger, 2016; Ertych et al., 2014; Schermuly, 2019).

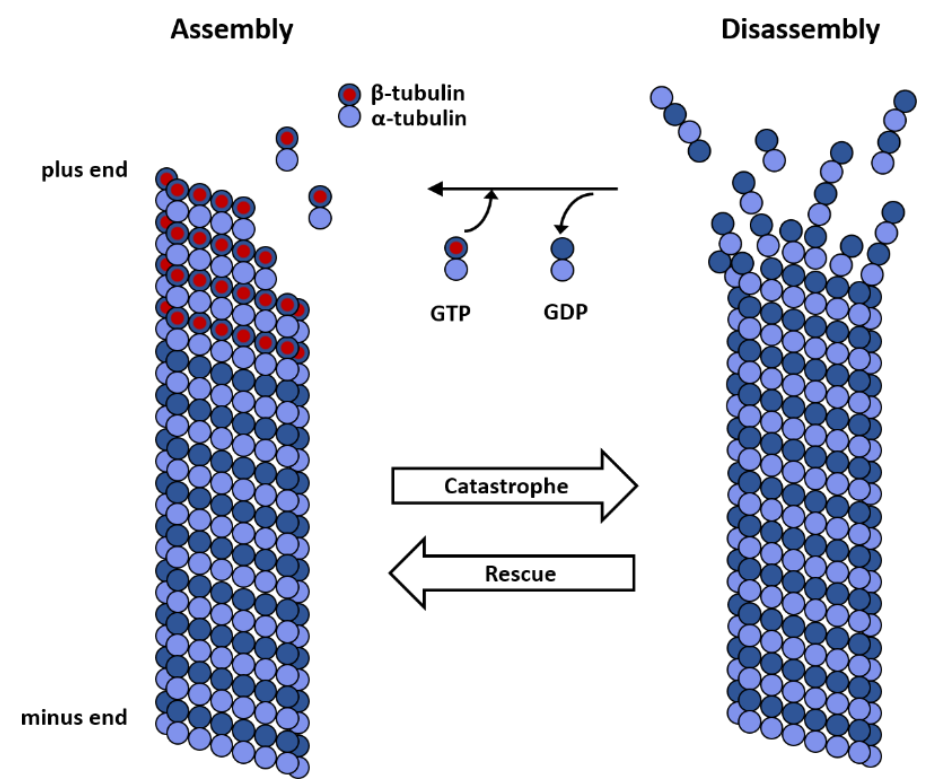

Figure 1.3: Dynamic instability of microtubules. Microtubules are assembled by polymerization of GTP bound $\alpha \beta$-tubulin heterodimers at the end of microtubules. When the addition of GTP bound tubulin at the microtubule end decreases, microtubule stability is reduced by ongoing GTP hydrolysis of assembled tubulin subunits resulting in microtubule disassembly. This process is called catastrophe, whereas a reactivation of microtubule growth is called rescue. Modified from Al-Bassam and Chang (2011).

\subsubsection{Assembly and regulation of the actin cytoskeleton}

Actin filaments are assembled by head-to-tail bound globular actin monomers (G-actin) (Alberts et al., 2015). Actin in its filamentous form is also called F-actin. Similar as for microtubules, the actin subunits all point in the same direction, thereby forming a filament with actin assembly and disassembly being fast at the plus-end (barded end) but slow at the minus-end (pointed end). Another impact on actin filament dynamics has the ATPase function of actin. Most free actin subunits are in an ATP bound form, that shows accelerated hydrolysis after incorporation into actin filaments. After hydrolysis, ADP remains stuck in the filament. Consequently, the actin filament 
exists in two structures: ATP bound actin filaments that are known to allow fast actin assembly and disassembly, and ADP bound actin filaments showing slower actin assembly and disassembly. In case the concentration of free actin subunits is within a specific range, assembly is faster than disassembly at the plus end, but not at the minus end, thereby keeping the plus end in ATP bound and the minus end in ADP bound conformation. This process, in which a continuous filament growth occurs at the plus end with simultaneous shrinkage at the minus end, is called treadmilling. However, not only the concentration of free actin subunits regulates actin filament dynamics, but also actin binding proteins (Pollard, 2016). While binding of thymosin to free actin subunits prevents actin assembly, profilin binding at the minus-end-faced site of actin subunits accelerates filament formation at the plus-end. Further regulation of actin filament dynamics is done by the F-actin capping protein (CP), that binds to plus-ends, thereby preventing assembly and disassembly of free actin subunits (Wear et al., 2003).

For actin filament formation, nucleation is required, which can take place by spontaneous assembly of actin monomers. However, small actin oligomers that form in this way are unstable and only few of them result in the assembly of a stable actin filament. For this reason, actin nucleation is mostly catalyzed by actin nucleators, such as the Arp2/3 complex, formins and tandem-monomer-binding nucleators (Firat-Karalar \& Welch, 2011). While the Arp2/3 complex promotes the formation of branched actin filaments in an ATP dependent manner, nucleation mediated by formins and tandem-monomer-binding nucleators result in unbranched, linear actin filaments (Figure 1.4) (Goley \& Welch, 2006). In order to generate branched actin filaments, the Arp2/3 complex attaches to the side of already existing actin filaments, resulting in the formation of a daughter filament branch that grows at an $70^{\circ}$ angle relative to the pre-existing mother filament. The Arp2/3 complex consists of seven subunits, from which the two actin-relating and heterodimer-forming proteins Arp2 and Arp3 are not only responsible for the name of the complex, but also show strong structural similarity to actin monomers that enables them to act as actin nucleator. From the five additional subunits ARPC1-5, the subunits ARPC2 and ARPC4 form a heterodimer that is responsible for the main interaction with the mother filament and generate the link between Arp3 and the daughter filament (Rouiller et al., 2008). This link is further supported by ARPC3. ARPC5 also interacts with the mother filament and stabilizes Arp2 within the complex. Although ARPC1 has only a minor important function in binding to the mother filament, it is essential for Arp2/3 complex activation by binding nucleation promoting factors (NPFs) (Pan et al., 2004). There are many NPFs known so far with the Wiskott-Aldrich Syndrome protein (WASP) family being the best-characterized group. The WASP family comprises WASP, N-WASP (neuronal WASP), SCAR/WAVE1-3 (suppressor of CAR/WASP and verprolin homologs 1-3), 
WASH (WASP and SCAR homolog), WHAMM (WASP homolog associated with actin, membranes and microtubules) and JMY (junction mediating regulatory protein) (Firat-Karalar \& Welch, 2011). All WASP family members contain a conserved VCA domain at their C-terminal regions, that contains an actin monomer binding verprolin homology sequence $(\mathrm{V})$ as well as central (C) and acidic sequences (A), that are able to bind to the Arp2/3 subunit ARPC1 (Kelly et al., 2006). Sometimes the VCA domain is also termed as WCA domain, with W standing for WASP homology 2 domain (Derivery \& Gautreau, 2010). The activity of WASP family members is regulated by an autoinhibitory conformation of the VCA domain that it has when it is not bound to an activating Rho (Ras homolog) family GTPase and in which VCA is not able to bind to the Arp2/3 complex (Padrick \& Rosen, 2010). While the Rho GTPase Rac1 activates SCAR/WAVE proteins by binding to their VCA domain, WASP is activated by VCA binding to Cdc42 (Goley \& Welch, 2006). Further activation of WASP family members is achieved by oligomerization of VCA domains, as this have been shown to result in a stronger Arp2/3 complex activation (Padrick \& Rosen, 2010).

In contrast to the formation of branched actin filaments, generated by Arp2/3 complex-mediated actin nucleation, formins promote formation of unbranched, linear actin filaments. The 15 formins known in mammals can be categorized into seven subtypes, namely Dia (diaphanous). DAAM (disheveled-associated activator of morphogenesis), delphilin, FHOD (fomin homology domaincontaining protein), FMN (formin), FRL (formin-related gene in leukocytes) and INF (inverted formin) (H. N. Higgs \& Peterson, 2005). All formins share a C-terminally located FH1 (formin homology 1) and FH2 (formin homology 2) domain. Dimerization of formin FH2 domains facilitates actin nucleation by binding to and thereby stabilizing spontaneously formed actin dimers or trimers (Goley \& Welch, 2006). In contrast to Arp2/3, formins protect the barbed ends from capping proteins as they remain associated with the growing barbed end after nucleation (Figure 1.4). Additionally, formins act as actin elongators by binding to profilin bound actin monomers via their $\mathrm{FH} 1$ domains, thereby bringing them close to the site of actin assembly at the barbed end (FiratKaralar \& Welch, 2011). Most mammalian formins belong to the Dia subtype, which contains a Rho GTPase binding domain (GBD), a diaphanous inhibitory domain (DID) and a diaphanous autoregulatory domain (DAD) (Courtemanche, 2018). Without binding of a Rho GTPase to the GBD, the interaction of DID and DAD results in autoinhibition of the formin. However, in case of Rho GTPase binding to the GBD, the DID/DAD association is disrupted and the autoinhibition is partially relieved. This partial activation is facilitated by the GTPases RhoA, RhoB, RhoC, Rac1 and Rac2 for the formin mDia1, whereas mDia2 requires binding of RhoA, RhoB, Rac1, Rac2 or Cdc42, and mDia3 interacts only with Cdc42 (Kühn \& Geyer, 2014). Further activation of formins 
requires additional factors, such as binding to specific membrane phospholipids (Ramalingam et al., 2010), interaction with NPFs or phosphorylation by kinases like the Rho-associated kinase (ROCK) (Breitsprecher \& Goode, 2013). Interestingly, several formins belonging to the Dia, FMN and INF subtypes have been shown to interact with microtubule plus-tip proteins, such as EB1, APC or the cytoplasmic linker protein 170 (CLIP170), or even directly with microtubules (Bartolini et al., 2008; Chesarone et al., 2010). Therefore, some formins do not only regulate actin filament formation but also microtubule stability and dynamics.

The group of tandem-monomer-binding actin nucleators includes Spire, cordon bleu (Cobl), leiomodin (Lmod), JMY and APC. All of them have tandem G-actin binding motifs that contain actin binding $\mathrm{WH} 2$ domains bringing together actin monomers to form an actin nucleus (FiratKaralar \& Welch, 2011). As the Arp2/3 complex, tandem-monomer-binding actin nucleators remain bound to the pointed end during actin filament elongation.

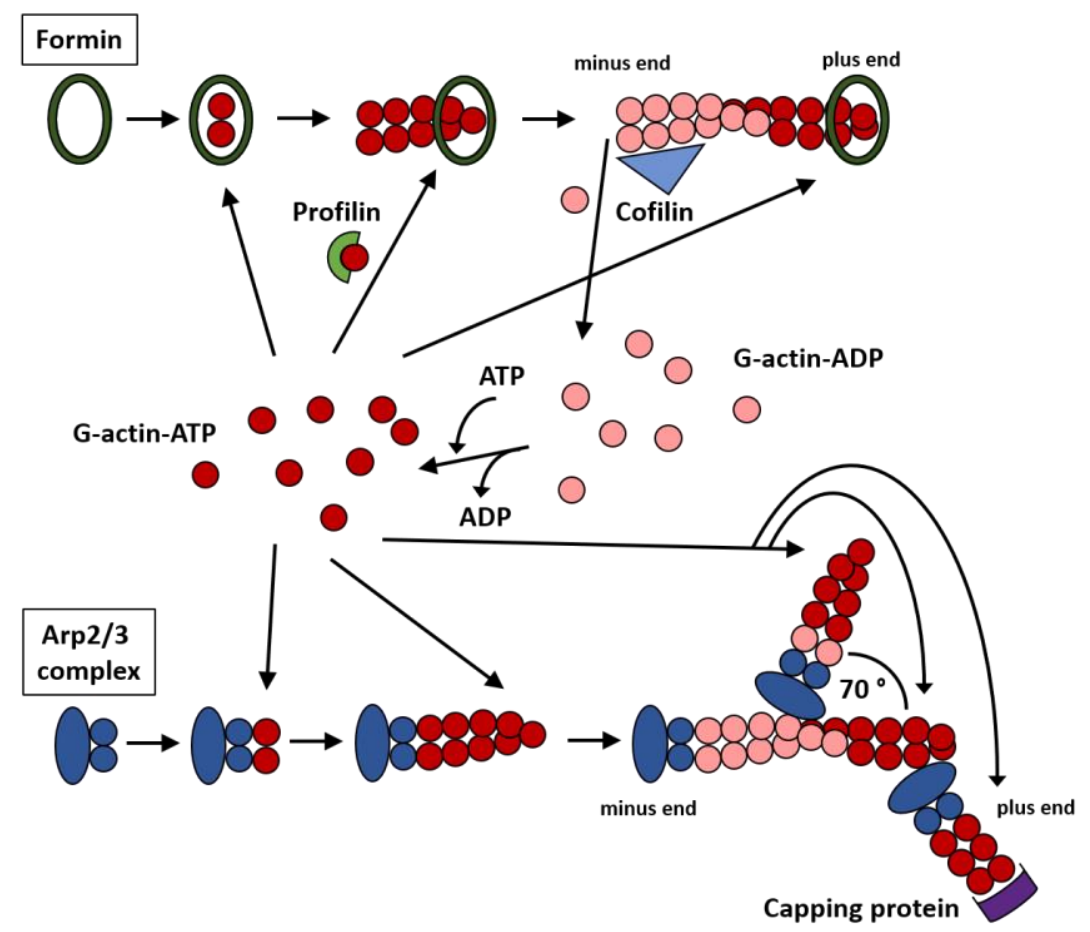

Figure 1.4: Actin filament assembly, nucleation and elongation factors. Cytoplasmic actin monomers (G-actin) are mostly present in GTP-bound form and assemble to actin filaments with the help of actin nucleators such as the Arp2/3 complex and formins. The Arp2/3 complex remains at the minus end after nucleation and enables formation of branched filaments, whereas formins stay associated with the growing plus-end and generate unbranched, linear filaments. Actin dynamics are regulated by several factors: cofilin binds to the minus ends and accelerates actin disassembly, whereas interaction of profilin with G-actin accelerates plus end assembly. By binding of the F-actin capping protein to the filament end, further growth is inhibited. Modified from Feher (2017). 
As mentioned above, Arp2/3 complex mediated nucleation results in the formation of unbranched actin filaments, while nucleation by formins and tandem-monomer-binding nucleators forms unbranched, linear filaments. Consequently, Arp2/3 organizes branched actin filaments into dendritic networks, whereas linear actin filaments nucleated by formins or tandem-monomerbinding nucleators are organized in parallel bundles (Davidson \& Wood, 2016). Another group of actin-binding proteins (ABPs), that act as crosslinkers, also affects actin filament organization. One of these crosslinkers is fimbrin that holds two actin filaments together in a parallel and very close manner via its directly adjacent actin binding sites, thereby generating tight actin bundles (Pollard, 2016). Actin filament crosslinking by fascin also results in tight actin bundles, but with antiparallel alignment. Another crosslinker, $\alpha$-actinin, forms a homodimer that contains two actin binding sites separated by a spacer, thereby forming more loosely packed, parallel actin bundles. The crosslinker filamin, however, forms homodimers with two V-shape-oriented actin binding sites that connect actin filaments into a loose, web-like network, in which the filaments are oriented in an almost $90^{\circ} \mathrm{C}$ angle to one another (Figure 1.5) (Nakamura et al., 2007). Depending on the crosslinker, interaction of other actin binding proteins with the crosslinked actin filaments can be affected. Fimbrin-mediated tight parallel actin bundles exclude binding of other proteins, such as a-actinin and myosin II (Alberts et al., 2015). The motor protein myosin II also acts as crosslinker

fimbrin
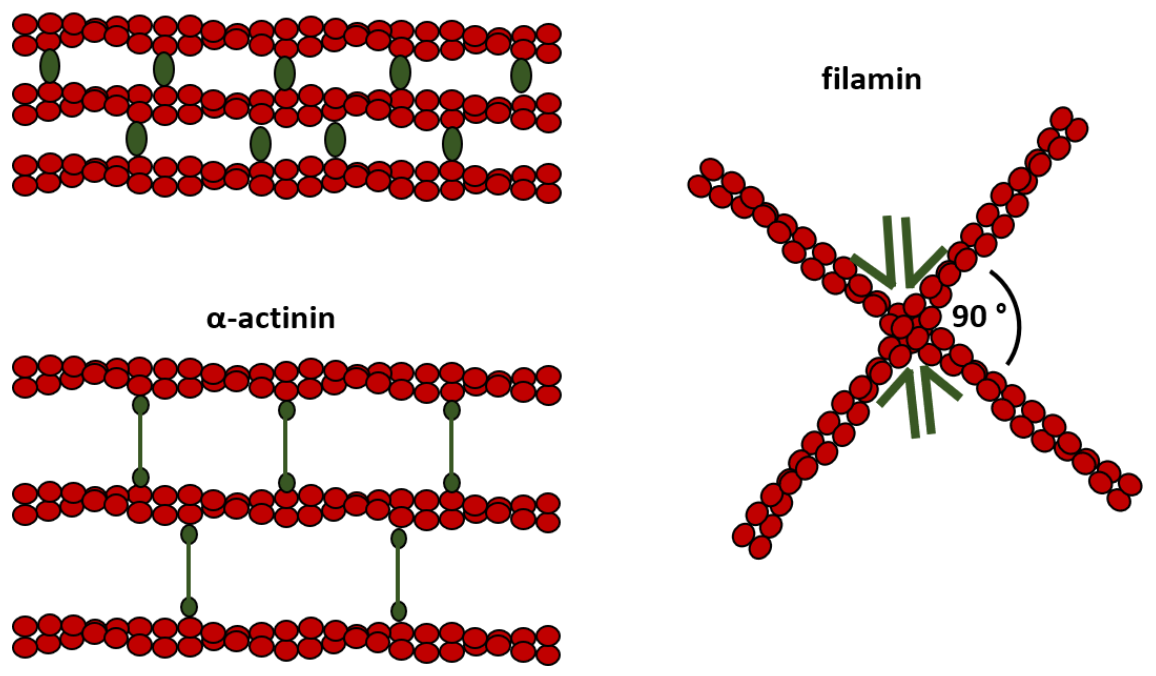

Figure 1.5: Actin filament crosslinker. The structural organization of actin filaments is dictated by crosslinking proteins: fimbrin contains two directly adjacent actin binding sites, thereby generating tight, parallel actin bundles. The two actin binding sites of $\alpha$-actinin are separated by a linker, resulting in more loosely packed, parallel actin bundles. Due to its $\mathrm{V}$-shape oriented actin binding sites, filamin connects actin filaments in an approximately $90^{\circ}$ angle, thereby creating a web-like actin network. Modified from Alberts et al. (2015). 
and is essential for the generation of a contractile actin structure by enabling actin filaments to slide relative to one another. In contrast to fimbrin, $\alpha$-actinin-mediated crosslinks result in a loose, parallel orientation of actin filaments that allows myosin II binding, thereby forming contractile actin bundles. Thick bundles of rigid, contractile actomyosin fibers are also called stress fibers. Depending on the type of organization, the resulting actin structure exhibits different mechanical properties that are required for different cellular processes, such as adhesion, migration, mitotic cell rounding and cytokinesis.

\subsubsection{Function and regulation of the cellular cytoskeleton in cell migration}

The ability to migrate is essential for many biological processes, such as embryonic development, immune surveillance and tissue repair. However, an impaired regulation of cell migration is known to promote progression of cancer cell invasion and metastasis (Fife et al., 2014; Yamaguchi \& Condeelis, 2007). In general, directed cell migration comprises several steps, beginning with forming an actin polymerization-driven protrusion of the cell membrane in the direction of movement that is determined by an extracellular gradient of cytokines or chemokines. In the next step, adhesion of the newly generated protrusions at the leading edge takes place by forming focal contacts that connect the actin cytoskeleton with extracellular matrix (ECM) proteins or with the substratum. Actomyosin-driven contraction at the rear part of the cell generates forces that trigger disassembly of cell-ECM adhesions at the cell rear (Lauffenburger \& Horwitz, 1996). This results in retraction of the trailing edge of the cell towards the direction of movement. In some cell types, disassembly of cell-ECM adhesions is accompanied by pericellular proteolytic degradation of ECM components (Friedl \& Wolf, 2009).

Actin polymerization-driven protrusions at the plasma membrane, which are generated at the leading edge of migrating cells, comprise lamellipodia, that are broad, sheet-like actin networks, and filopodia, which are thin, finger-like projections of crosslinked actin bundles extending beyond the lamellipodia (Yamaguchi \& Condeelis, 2007). These differences in shape are based on different actin filament nucleators and crosslinker: While the actin network of lamellipodia depends on Arp2/3, tight actin bundles of filopodia are nucleated by mDia formins and mostly crosslinked by fimbrin or fascin. A timely and spatially coordinated assembly and disassembly of actin structures required for the formation of adhesion sites, lamellipodia and filopodia is crucial for cell migration (Lawson \& Ridley, 2018; K. Martin et al., 2016). This tight regulation of actin dynamics depends on several members of the family of Rho GTPases. While Cdc42 promotes mDiamediated formation of actin filaments in filopodia, Rac1 is required to induce Arp2/3-mediated lamellipodia formation (Hall, 1998). RhoA is present at the front of lamellipodia, where it is thought 
to activate the formin mDia (A. J. Ridley, 2015). In addition, RhoA activates ROCK, especially at the rear of the migrating cell. ROCK in turn triggers myosin II-dependent contraction by increasing the phosphorylation of the myosin light chain (MLC) on the one hand by its kinase function and on the other hand by inhibiting MLC phosphatase (A. J. Ridley, 2001). ROCK also activates LIM kinase (LIMK), which in turn inhibits the actin binding protein cofilin, that is known to disassemble actin filaments at their minus-ends (Yamaguchi \& Condeelis, 2007). LIMK is not only activated by ROCK, but also by the actin-binding protein p21-activated kinase (PAK), that is again activated by Rac1 and Cdc42. Another effector of both Rac1 and Cdc42 is the actin binding protein IQGAP1, that is not only able to modulate actin dynamics by itself, but also by activating N-WASP, which then activates the Arp2/3 complex, and by activating formins (Watanabe et al., 2015). Furthermore, Rac1 was found to promote actin polymerization by stimulating phosphatidylinositol4-phosphate 5-kinase (PI(4)P 5-kinase), that catalyzes the generation of phosphatidylinositol-4,5bisphosphate $\left(\mathrm{PI}(4,5) \mathrm{P}_{2}\right)$, which in turn was shown to cause capping protein disassembly from barbed plus ends (Figure 1.6) (Tolias et al., 2000).

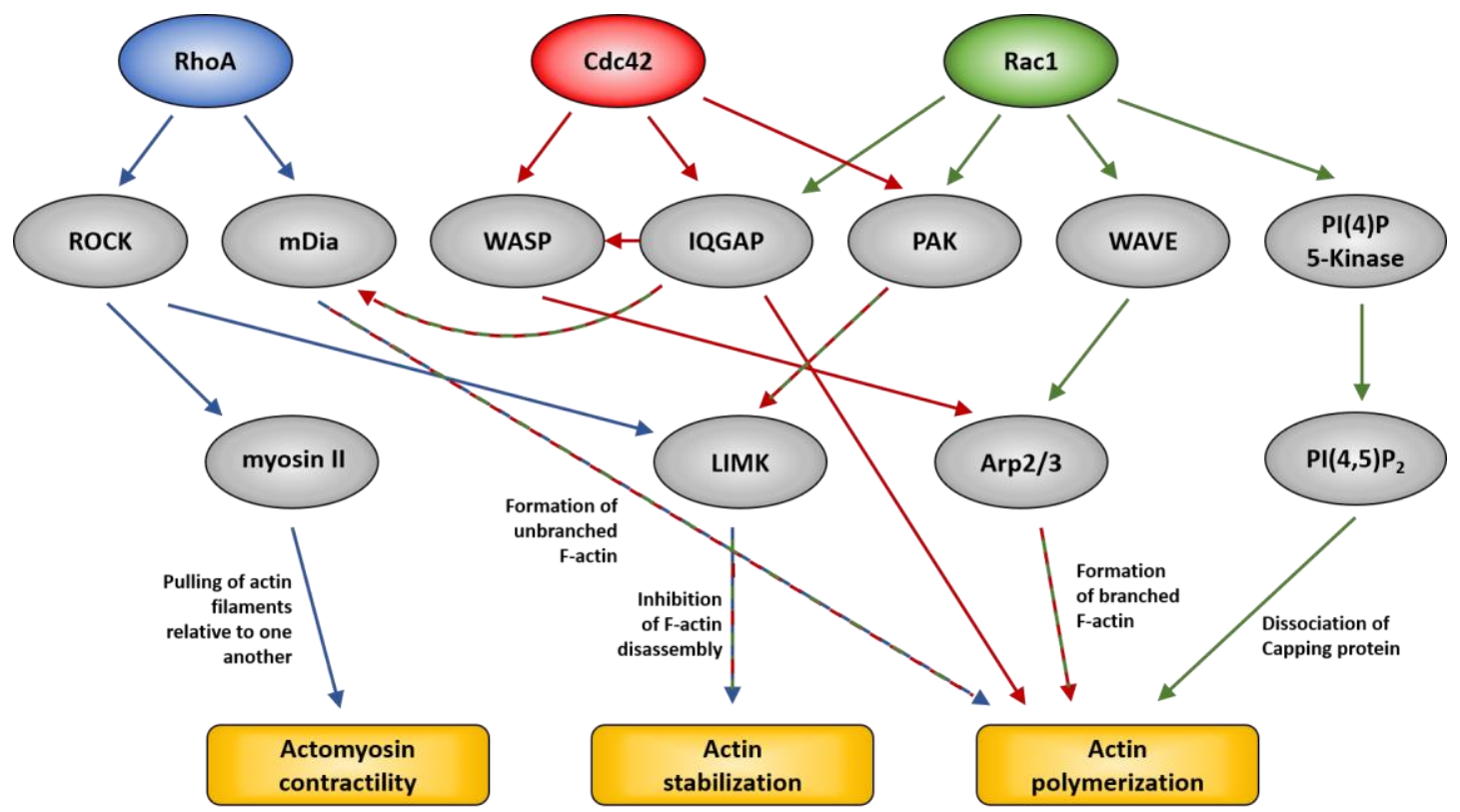

Figure 1.6: Regulation of actin cytoskeleton dynamics by Rho GTPases and their effectors. RhoA activates ROCK, that triggers actomyosin contractility via myosin II activation. Activation of mDia by RhoA results in the generation of unbranched actin filaments. Cdc42 and Rac1 activate the Arp2/3 complex via the WASP and WAVE complexes, respectively. This leads to the formation of branched actin filaments. Cdc42 and Rac1 also activate IQGAP, that stimulates actin polymerization via activation of N-WASP, Arp2/3 and formins, such as mDia. Another Cdc42 and Rac1 target is PAK, that triggers activation of LIMK, which in turn prevents disassembly of actin filaments via inhibition of cofilin. Actin polymerization is also triggered by Rac1 dependent activation of $\mathrm{PI}(4) \mathrm{P} 5$-kinase, that promotes $\mathrm{PI}(4,5) \mathrm{P}_{2}$ formation, which in turn mediates the dissociated of capping protein from plus ends. Modified from A. J. Ridley (2001) and Millar et al. (2017). 
This complex, interwoven signaling pathways demonstrate the importance of the family of Rho GTPases in regulatory processes of cell migration. In turn, the Rho GTPases participating in these processes are regulated by opposing actions of GTPase-activating proteins (GAPs) and guanine nucleotide exchange factors (GEFs), as well as by guanine nucleotide dissociation inhibitors (GDIs) (Lawson \& Ridley, 2018). GAPs are proteins that bind to GTPases, such as Rho GTPases, and induce hydrolyzation of bound GTP, thereby driving the protein in the inactive, GDP bound state (Alberts et al., 2015). At the opposite site, GEFs promote the release of bound GDP. As the empty nucleotide-binding site immediately binds a free GTP molecule, GEFs indirectly activate the GTPase. Active Rho GTPases need to be membrane-associated. They reside and function mainly at the plasma membrane, but a part of Rho GTPase proteins is localized to intracellular endomembranes (Phuyal \& Farhan, 2019). GDls do not only inhibit activation of GTPases by preventing the dissociation of GDP from the GTPase, but also by preventing the GTPases from membrane-association (Figure 1.7) (Biro et al., 2014).

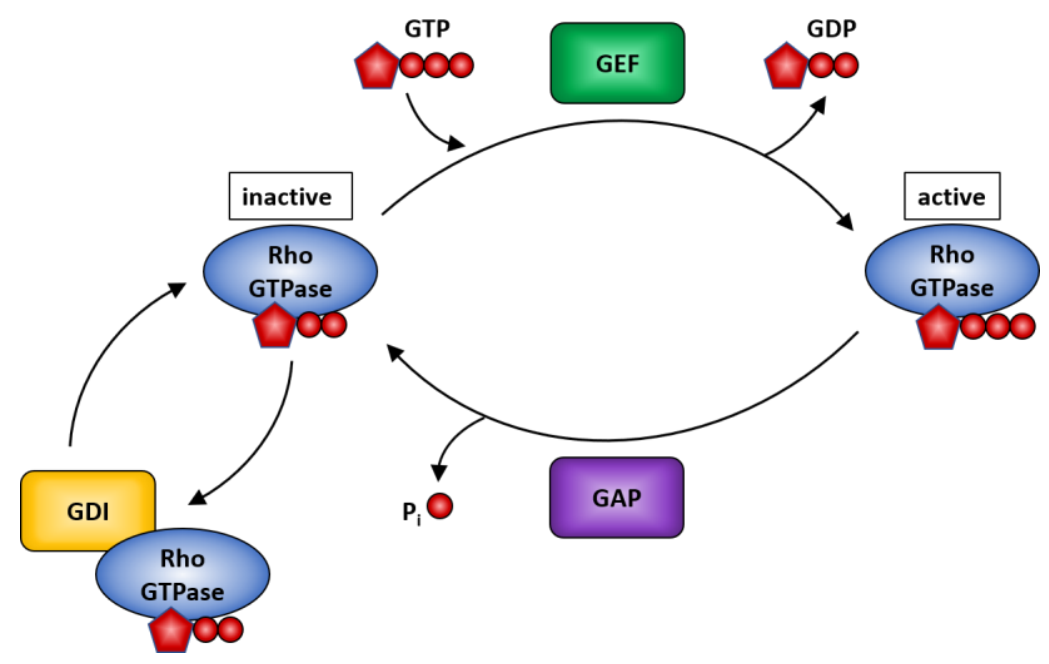

Figure 1.7: Regulation of Rho GTPases by GEFs, GAPs and GDIs. Inactive GDP-bound Rho GTPases are activated by GEFs through catalyzation of GDP to GTP exchange. GAPs in turn catalyze hydrolysis of GTP bound to Rho GTPase resulting in its inactivation. Binding of GDIs to Rho GTPases prevents dissociation of GDI and thus Rho GTPase activation. Modified from Kim et al. (2019).

Many GEFs and GAPs are known that are involved in the regulation of Rho GTPase-mediated migration (Lawson \& Ridley, 2018). In general, there are two subtypes of GEFs activating Rho GTPases (RhoGEFs): the DOCK and the Dbl family. All 11 human DOCK GEFs contain a DOCK homology region 2 (DHR2), that catalyzes guanine nucleotide exchange, and a DOCK homology region 1 (DHR1), that binds to plasma membrane-associated phospholipids. The human Dbl family is composed of about 70 GEFs, that all share a catalytically active Dbl homology (DH) domain stimulating guanine nucleotide exchange, and a pleckstrin homology $(\mathrm{PH})$ domain that is 
involved in GEF activation and localization (A. Schmidt \& Hall, 2002). The well-characterized GEF $\beta$-PIX belongs to the Dbl family of Rho GEFs and is able to activate Rac1 and Cdc42, depending on the bound co-factor. When bound to Rac1, $\beta$-PIX triggers focal adhesion turnover, while its activation of Cdc42 induces leading edge polarization. In complex with diverse co-factors, the Dbl family GEF TIAM1 (T-lymphoma invasion and metastasis inducing protein 1) activates Rac1, thereby promoting multiple cell type and context dependent changes in the actin cytoskeleton: on the one hand, TIAM1 was shown to inhibit cell migration by enhancing cell-cell adhesion. On the other hand, TIAM1 is involved in lamellipodium extension, thereby stimulating migration (Figure 1.9). Another Dbl family Rho GEF that is involved in the regulation of migratory processes is the triple functional domain protein (TRIO) (S. Schmidt \& Debant, 2014). Together with its paralog Kalirin, TRIO is the only Dbl family member that contains two GEF domains responsible for the activation of distinct Rho GEFs. The GEF-D1 domain activates the two GTPases Rac1 and RhoG, while the GEF-D2 domain specifically activates RhoA. Additionally, TRIO harbors a serine kinase domain, so that the name of TRIO refers to the fact that is possesses three catalytic domains (Figure 1.8). The PH domain of TRIO GEF-D1 is not only positively involved in the catalytic reaction, but also binds to the actin filament crosslinker filamin $A$, thereby targeting TRIO to the actin cytoskeleton (Bellanger et al., 2000). In contrast, the RhoA-activating PH domain of TRIO GEF-D2 has an inhibitory effect on the GEF-D2 catalytic reaction, so that it requires activating factors, from which Gaq is one (Rojas et al., 2007). Interestingly, upon activation by Gaq, TRIO GEF-D1 activated Rac1 as well as TRIO GEF-D2 activated RhoA were shown to stimulate mitosisinducing (mitogenic) signaling via the MAPK family members (mitogen-activated protein kinase) JNK (Jun N-terminal kinase) and p38 (Vaqué et al., 2013). Consequently, overexpression of TRIO was found to result in sustained activation of mitogenic signaling resulting in aberrant cell proliferation. Moreover, increased mitogenic signaling, such as induced by JNK, was found to promote genomic instability as it promotes unscheduled activation of replication and transcription (Chen et al., 2010; Tubbs \& Nussenzweig, 2017). This causes replication and transcription stress, resulting in the generation of DNA double strand breaks and SSDNA and thereby structural chromosome aberrations.

In addition to its well-studied function as regulator of axon guidance in neuronal development, the ubiquitously expressed TRIO was also shown to regulate cell migration by stimulating Rac1dependent lamellipodia formation (van Rijssel et al., 2012), as well as the formation of integrinmediated cell-ECM adhesions, but it also participated in the generation of cadherin-mediated cellcell adhesions (Figure 1.9) (S. Schmidt \& Debant, 2014). To enable cell migration, cadherinmediated cell-cell adhesions are resolved, which requires local inhibition of TRIO by interaction 
with the F-actin binding protein Tara (TRIO-associated repeat on actin). TRIO is not only located at the actin cytoskeleton via binding of filamin $A$, but also at the plasma membrane by binding to the transmembrane tyrosine phosphatase LAR (van Rijssel \& van Buul, 2012). Additionally, TRIO was found to localize to microtubule +TIPs by interacting with the EB1-binding protein Nav1 (Navigator 1) and by direct binding of TRIO to EB1 via one of its two SXIP motifs (van Haren et al., 2014). Reducing the amount of TRIO bound to +TIPs by Nav1 knock down resulted in decreased Rac1 activity, thereby proposing a +TIP dependent role for TRIO-mediated Rac1 activation.

Due to their strong impact on cell migratory processes, high levels of Rho GTPases, especially RhoA, Rac1 and Cdc42, and of GEFs, such as TIAM1 and TRIO, are associated with tumor progression and metastasis in different human tumors, including melanoma, liver cancer, breast cancer, testicular cancer and ovarian cancer (Ellenbroek \& Collard, 2007; Haga \& Ridley, 2016; Lane et al., 2008; Wang et al., 2015).

Although it is known for long, that the actin cytoskeleton plays a major role in cell migration, the microtubule cytoskeleton is also of particular importance for this process (Garcin \& Straube, 2019; Kaverina \& Straube, 2011). Consequently, deregulation of microtubule dependent processes during cell migration induces cancer progression and metastasis. Microtubules provide an

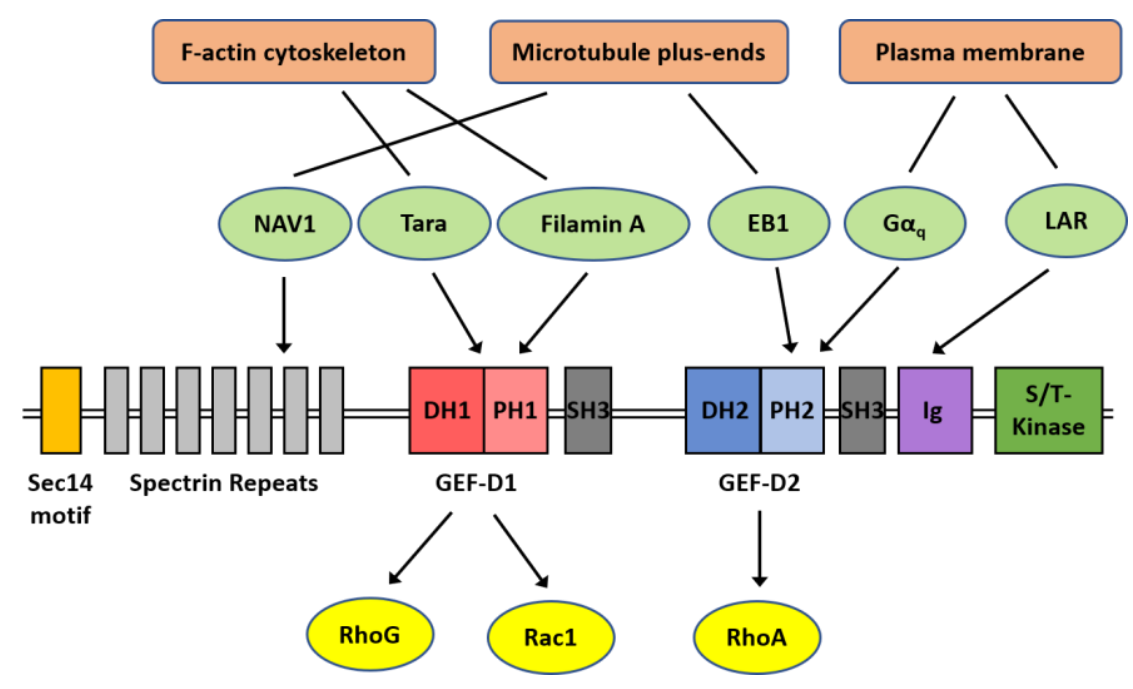

Figure 1.8: The multidomain protein TRIO and its functional domains. TRIO contains two GEF domains, GEF-D1 for the activation of RhoG and Rac1 and GEF-D2 for RhoA activation. The third catalytically active domain is a serine/threonine kinase domain at the $\mathrm{C}$-terminus. Due to various interaction partners, TRIO localizes to the F-actin cytoskeleton via Tara and filamin A, to microtubule plus-ends by binding to NAV1 and EB1, and to the plasma membrane by interacting with LAR and Gaq. Moreover, TRIO harbors a N-terminal Sec14 motif, several spectrin-like repeats, two SH3 domains and an Immunoglobulin (Ig) domain. Modified from S. Schmidt and Debant (2014). 
intracellular transport network, that allows rapid and directed transport of membrane vesicles containing cytoskeletal components, signaling molecules and mRNAs resulting in the required front/back polarity between the leading edge and the rear of a migrating cell. Not only the directed, microtubule-associated transport of membrane vesicles generates cell polarity, but also microtubule + TIP protein complexes that assemble at growing microtubule plus-ends and that carry signaling molecules acting as regulators for microtubule and actin dynamics. Additionally, microtubules are involved in the formation of membrane protrusions due to their physical properties, that make them stable against compressive forces and at the same time enable them to generate pushing forces. Moreover, microtubules exhibit pulling forces that facilitate movement of the nucleus and the centrosome during cell migration. Due to all these functions, microtubule dynamics mediate cell migration by regulating actin dynamics and Rho GTPase signaling, as well as focal adhesion turnover.

In order to generate cell polarity in a migrating cell, spatially different modulation of actin and microtubule dynamics is required. Microtubules are selectively destabilized at the cell rear, whereas activities at the lamellipodial leading edge promote microtubule stabilization and growth (Kaverina \& Straube, 2011; Torsten Wittmann et al., 2003). While the microtubule depolymerase MCAK triggers microtubule disassembly mainly at the cell rear (Braun et al., 2014), microtubule stability is increased at the leading edge by + TIP complex proteins, such as CLIP170, CLASPS, APC and EBs, which link microtubule plus-ends to the cell cortex (Akhmanova \& Steinmetz, 2008; Henty-Ridilla et al., 2016). Rho GTPase activated mDia formins have also been shown to be involved in + TIP protein-induced microtubule stability, due to their interaction with EB1, APC and CLIP170 (Henty-Ridilla et al., 2016; Wen et al., 2004). Moreover, Rac1 and Cdc42 activated IQGAP1 was reported to interact with the +TIPS CLIP170, CLASPs and APC, resulting in the induction of Arp2/3 and formin dependent formation of actin filaments at the leading edge (Kaverina \& Straube, 2011). Rac1 and Cdc42 were also shown to have an effect on microtubule dynamics by inducing microtubule growth via their effector PAK, that phosphorylates and thereby inactivates the tubulin polymerization preventing protein stathmin (Daub et al., 2001; Torsten Wittmann et al., 2003). Interestingly, microtubule plus-end growth was shown to vice versa induce Rac1 activity, resulting in actin polymerization in lamellipodial protrusions of migrating cells (Waterman-Storer et al., 1999). Looking for the link between microtubule plus-end growth and Rac1 activity in migrating cells, the GEF STEF (Sif and TIAM1-like exchange factor) was identified. STEF activates Rac1 in a microtubule growth dependent manner, thereby inducing 


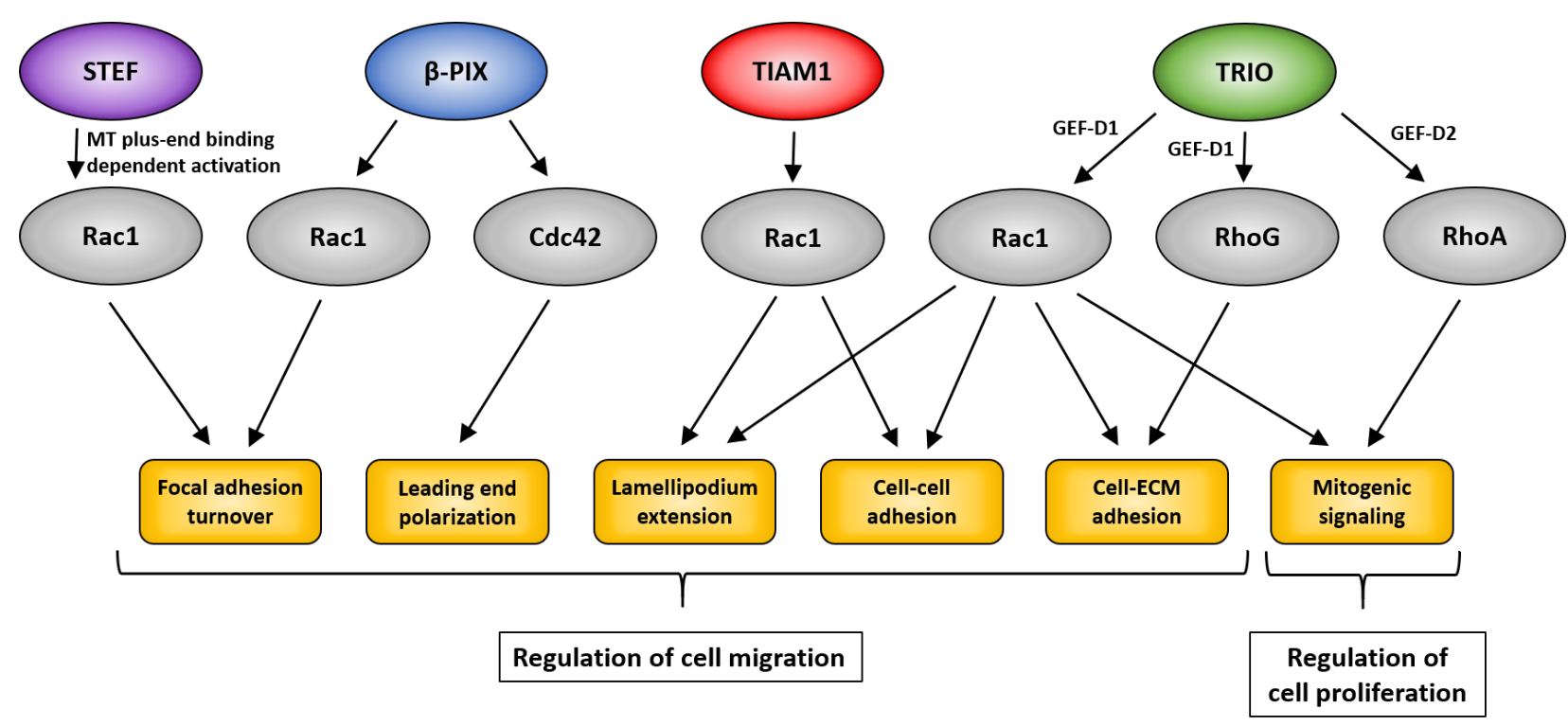

Figure 1.9: Involvement of GEFs and their Rho GTPases in cell migration and proliferation. The GEFs STEF and $\beta$-PIX activate Rac1 to promote focal adhesion turnover, thereby inducing cell migration. $\beta$-PIX also activates Cdc42, that triggers polarization of the cell's leading edge. The GEFs TIAM1 and TRIO activate Rac1 to induce actin polymerization at the lamellipodium, but also cell-cell adhesion. For cell migration, Rac1-mediated cell-cell adhesion is decreased by local TIAM1 and TRIO inhibition. TRIO also controls cell-ECM adhesion by activating Rac1 and RhoG and promotes mitogenic signaling through activation of Rac1 and RhoA. Modified from S. Schmidt and Debant (2014) and Lawson and Ridley (2018).

focal adhesion turnover (Rooney et al., 2010). The GEF TRIO, that is known to also localize to microtubule plus-ends via EB1 and Nav1, induces cell migration especially by triggering Rac1dependent actin polymerization in lamellipodial protrusions (Figure 1.9) (S. Schmidt \& Debant, 2014; van Rijssel et al., 2012). However, although it was reported that neuronal axon outgrowth is induced by TRIO-mediated Rac1 activation in a microtubule plus-end binding dependent manner, it seems that this binding is not required for TRIOs effect on cell migration (van Haren et al., 2014).

\subsubsection{Structure and function of the actin cytoskeleton in mitosis}

At the onset of mitosis, cells need to round up to generate an appropriate geometry for proper mitotic spindle alignment and function as well as cleavage furrow positioning required for errorfree chromosome segregation and cell division (Cadart et al., 2014; Lancaster et al., 2013; Théry \& Bornens, 2006). In order to facilitate mitotic rounding, the actin cytoskeleton undergoes profound reorganization (Théry \& Bornens, 2008). This especially includes disassembly of stress fibers, which span the cytoplasm of interphase cells and which are necessary for the formation of cell- 
cell adhesions via adherens junctions and of cell-ECM adhesions via focal adhesions (Tojkander et al., 2012). Following disassembly of stress fibers and adhesion sites, only actin- and myosin IIrich retraction fibers keep the mitotic cell attached and in correct spatial arrangement required for proper mitotic spindle positioning (Fink et al., 2011; Mitchison, 1992; Théry et al., 2005). Rounding of the cell is mediated by increasing the intracellular, hydrostatic pressure as well as the tension of the mitotic cortex by actomyosin-driven contractility (Cadart et al., 2014; Lancaster \& Baum, 2014; Stewart et al., 2011). While the intracellular, hydrostatic pressure is regulated by plasma membrane transporters, cortical tension depends on myosin II, which creates contractile forces by pulling actin filaments relative to one another, but also on the cortical actin network architecture (Figure 1.10) (Chugh et al., 2017; Ramanathan et al., 2015). To generate an increase in cortex tension detected in mitotic cells, myosin II accumulates at the cortex and is activated via the RhoAROCK pathway (Maddox \& Burridge, 2003; Théry \& Bornens, 2008). Interestingly, activation of RhoA was shown to be driven by Cdk1 via its downstream target and Rho GEF Ect2 (Matthews et al., 2012; Ramanathan et al., 2015). In addition, the cortical actin network of mitotic cells involves the actin nucleators Arp2/3 and mDia1 (Bovellan et al., 2014), as well as the actin crosslinkers $\alpha$-actinin and fascin (Toyoda et al., 2017). Moreover, F-actin capping protein as well as cofilin participate in the regulation of mitotic cortex architecture (Chugh et al., 2017). Deregulation of the actin cortex structure was shown to result in decreased cortex tension. On the one hand, cortical tension is modulated by the thickness of the actin cortex, which is determined by actin filament length regulators, such as mDia, capping protein and cofilin: Depletion of the actin filament elongator mDia1 results in short actin filaments and a reduced cortex thickness, that is missing enough actin filament connections required for increased cortex tension. However, depletion of capping protein and cofilin leading to the formation of long actin filaments and a thick, overly rigid actin cortex also causes decreased cortex tension. Consequently, proper mitotic cortex tension requires the actin cortex to contain actin filaments of intermediate length, resulting in a defined cortex thickness. On the other hand, cortex tension is also modulated by the cortical actin network connectivity, that is determined by actin filament crosslinkers, such as Arp2/3 and $\alpha$ actinin (Ennomani et al., 2016). A highly connected actin network exhibits only low contractility, that can be increased by debranching. Debranching of an actin network with optimal connectivity, however, reduces contractile forces. Summed up, the composition and organization of the mitotic actomyosin cortex need to be tightly balanced for the generation of sufficient contractile forces required for an increase in cortical tension. However, mechanisms that contribute to a tensiongenerating reorganization of cortical actin structures remain largely unknown. 


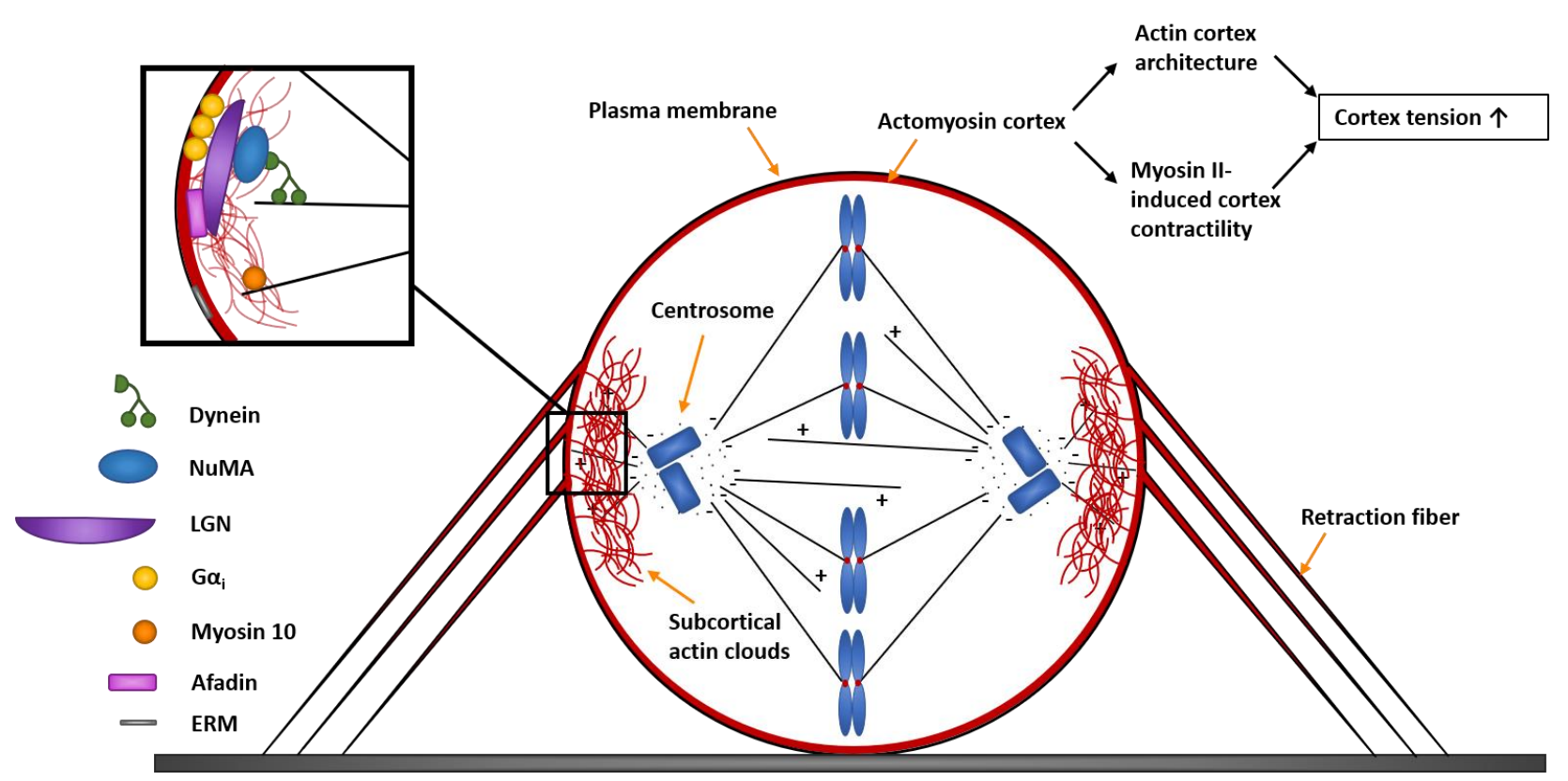

Figure 1.10: The role of the actin and microtubule cytoskeleton in mitotic spindle positioning. Astral microtubules link the spindle pole to the cortex via bound dynein, that interacts with the plasma membrane anchored NuMA-LGN-Gai complex. LGN also generates a connection to the actin cortex by interacting with the actin binding protein Afadin. In addition, astral microtubules transmit the force, which is generated by retraction fibers, to centrosomes via myosin 10-mediated interaction with subcortical actin clouds. Moreover, a proper spindle positioning requires an ERM-mediated connection between the plasma membrane and the actin cortex. Additionally, mitotic cell rounding, that is essential for correct spindle positioning, is generated by an increase in cortical tension, which in turn requires myosin II dependent cortex contractility and a proper actin cortex architecture. Modified from Di Pietro et al. (2016).

Cortex tension seems to be important not only for mitotic cell rounding, but also for migration and invasion, as clinical studies revealed a decreased stiffness for metastatic cancer cells of different types and cell lines when compared to benign cells (Cross et al., 2007; Remmerbach et al., 2009; Swaminathan et al., 2011).

In addition to the role of the actin cytoskeleton within the process of cell rounding, it was also found to form dynamic, subcortical clusters of Arp2/3-dependent actin filaments, so-called actin clouds, during mitosis (Mitsushima et al., 2010). These have further been shown to mediate mitotic spindle orientation by forwarding pulling forces generated from retraction fibers to centrosomes via myosin 10 (Myo10)-mediated binding to astral microtubules (Kwon et al., 2015). Ablation of retraction fibers impairs subcortical actin cloud dynamics and results in spindle alignment defects (Fink et al., 2011). Since spindle positioning defects have been associated with chromosome missegregation, deregulation of the structure or dynamics of mitotic, subcortical actin clouds might also be involved in the induction of chromosomal instability (Ertych et al., 2014). 


\subsection{The mitotic spindle}

During mitosis, not only tight regulation of the actin cytoskeleton is essential for proper spindle positioning and chromosome segregation, but in particular also the assembly of a bipolar microtubule-based mitotic spindle (Prosser \& Pelletier, 2017). The trigger for mitotic spindle assembly is given by the formation and activation of the cyclin $\mathrm{B} / \mathrm{CDK} 1$ complex at the end of $\mathrm{G}_{2}$ phase. In general, a mitotic spindle consists of an antiparallel array of microtubules being anchored at the two spindle poles by their minus ends and radiating outwards from the poles with their plus ends (T. Wittmann et al., 2001). Microtubules within this array, whose plus ends are attached to the kinetochores from sister chromatids, are called kinetochore microtubules, whereas the plus ends of so-called interpolar microtubules overlap with plus ends from microtubules emanating from the other pole. For a correct positioning of the spindle within the round mitotic cell, astral microtubules connect the spindle poles with the cell cortex, that is defined as a layer of an actomyosin network bound to the inner surface of the plasma membrane. In mitotic vertebrate cells, the two spindle poles are associated with the centrosomes, that are each composed of two centrioles surrounded by amorphous pericentriolar material (PCM), and that drive spindle assembly by their function as main microtubule organization center (MTOC). The MTOC is important for $y$-tubulin-driven microtubule nucleation, but also for microtubule anchorage and arrangement. However, higher plant cells as well as meiotic cells lack centrosomes, so that bipolar spindle assembly is mediated acentrosomally. Centrosome containing cells were also shown to still form bipolar mitotic spindles after centrosome disassembly by ablation or removal of centrioles, thereby proving centrosome independent spindle assembly pathways to exist not only in centrosome-lacking cells (Khodjakov et al., 2000).

\subsubsection{Chromosome dependent and independent assembly of the mitotic spindle}

By today, spindle assembly has been shown to be mediated by one centrosome dependent and several centrosome independent pathways. In cells containing centrosomes, these are duplicated at about the time of DNA replication (T. Wittmann et al., 2001). The resulting centrosome pair is connected by centrosomal linker proteins and remains at the outer side of the nuclear envelope until mitotic entry. When cells enter mitosis, the amount of $\mathrm{y}$-TurC complexes at the centrosomes increases, thereby promoting massive microtubule nucleation. Additionally, microtubule dynamics increase greatly, resulting in microtubule plus ends rapidly changing between growth and shrinkage. Moreover, the centrosomal linker is dissolved and the centrosomes are separated by the activity of the plus-end directed kinesin- 5 motor protein Eg5, which is activated by CDK1 


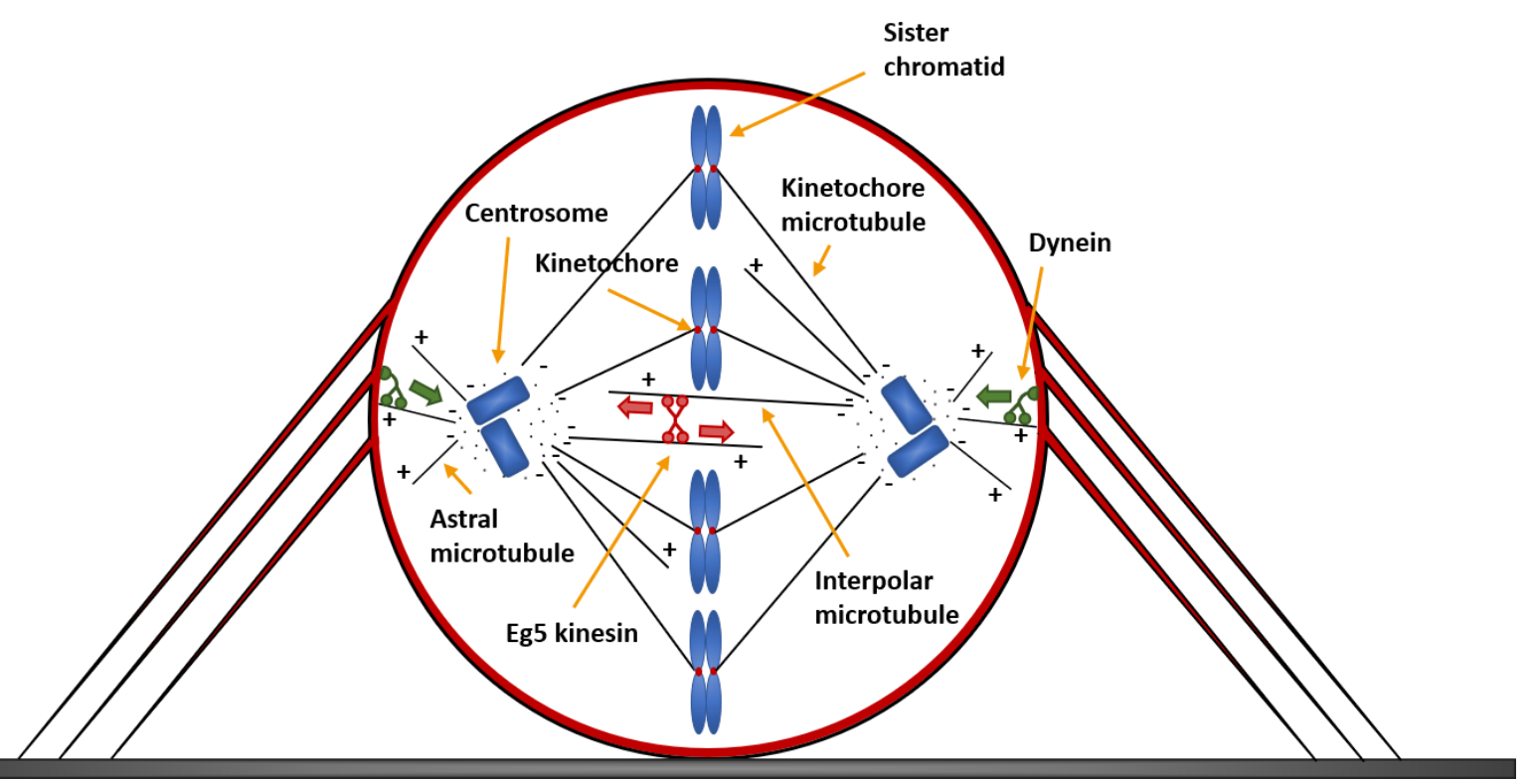

Figure 1.11: Assembly of the mitotic spindle. Upon entry into mitosis, centrosome dependent and independent (not shown) pathways induce massive microtubule nucleation. Centrosomes are pushed apart via activity of plus-end directed Eg5 kinesin on microtubules emanating from opposing spindle poles. Additionally, nuclear envelope-associated dynein (before NEBD, not shown) and cortex-associated dynein pull microtubules and thus the centrosomes towards opposing poles. Microtubules that connect the spindle poles with the cortex are called astral microtubules, while interpolar microtubules overlap with plus ends from microtubules emanating from the other pole and kinetochore microtubules are attached to kinetochores with their plus end. Modified from Prosser and Pelletier (2017).

activity, and the minus-end directed motor protein dynein (Agircan et al., 2014; Tanenbaum \& Medema, 2010; van Heesbeen et al., 2013). Eg5 crosslinks and moves apart antiparallel microtubules emanating from opposing centrosomes, resulting in centrosome separation along the nucleus. Nuclear envelope-associated dynein pulls the centrosomes further apart. Microtubules that grow towards the periphery are captured and linked to the cell cortex by cortical dynein, which pulls the centrosomes to opposing poles of the cell (Figure 1.11). Following nuclear envelope breakdown (NEBD), further centrosome separation and spindle positioning is driven by astral microtubules that are connected to and move along the cortex via localized cortical actomyosin contractions (Rosenblatt et al., 2004). Again, dynein was shown to generate the required link between astral microtubules and the actomyosin cortex (Simone et al., 2016).

When centrosomes are present in a cell, they provide the dominant microtubule nucleation and organization site (Heald et al., 1997). However, there is also a chromosome-based selforganization process going on in a mitotic cell, that acts as major driver for spindle assembly in most acentrosomal cells. This process involves the GTPase Ran, known to participate in 
nucleocytoplasmic transport by binding to nuclear transport receptors (NTR). Ran activity is controlled by its locally separated regulators, the chromatin-bound GEF RCC1 (regulator of chromatin condensation 1) and the cytoplasmic GAP RanGAP (Kalab \& Heald, 2008). Due to the different localization of its regulators, RanGTP is formed at a higher rate around chromosomes and is rather converted to RanGDP in the cytoplasm. Even after NEBD, there are still high RanGTP concentrations around the chromosomes decreasing with increasing distance from the chromosomes. This RanGTP gradient goes along with a gradient of RanGTP-bound NTRs and their cargo proteins. These cargo proteins especially include spindle assembly factors, that recruit microtubule nucleating $\mathrm{Y}$-TurC complexes to the proximity of chromosomes or stabilize microtubules, such as the cyclin L/CDK11 complex (Forbes et al., 2015; Yokoyama et al., 2008). Further stabilization of microtubules, that have been nucleated by $\mathrm{Y}$-TurC complexes, takes place at kinetochores and depends the chromosomal passenger complex (CPC) and its component Aurora B kinase, which both are localized at centromeres during mitosis (Meunier \& Vernos, 2016). Aurora B phosphorylates and thus inactivates the microtubule depolymerase MCAK and the protein stathmin, which is known to prevent tubulin polymerization.

Acentrosomal spindle assembly is not only triggered by chromosomes via the RanGTP gradient and the CPC, but also by an augmin dependent pathway that induces microtubule nucleation and amplification depending on microtubules themselves. The augmin complex recruits $\gamma$-TurC to the side of a pre-existing microtubules, resulting in the nucleation of a new, branched microtubule, that is transported along the pre-existing microtubule by dynein and Eg5 towards the spindle poles (Lecland \& Lüders, 2014). The chromosome dependent pathway and the chromosomeindependent pathways are not mutually exclusive, but function together in the assembly of the mitotic spindle (Prosser \& Pelletier, 2017).

\subsubsection{Orientation of the mitotic spindle}

The orientation of the mitotic spindle is not only essential for the maintenance of the tissue architecture within an epithelium, but also plays an important role for proper chromosome alignment and segregation, as spindle mis-positioning is associated with chromosome missegregation and chromosomal instability (Ertych et al., 2014; Silkworth \& Cimini, 2012). As described above, a proper spindle positioning requires not only an increase in cortex tension to round up (Kunda et al., 2008), but also a complex interplay between the actin and microtubule cytoskeleton: astral spindle microtubules have to be linked to the actomyosin cortex by dynein (Tanenbaum \& Medema, 2010) and they have to transmit the force generated by retraction fibers to centrosomes via myosin 10-mediated interaction with subcortical actin clouds (Kwon et al., 
2015). The dynein-mediated crosslink between astral microtubules and the actin cortex involves a complex consisting of several proteins: close to the inner cortex surface, microtubule-bound dynein interacts with NuMA (nuclear mitotic apparatus protein). NuMA binds to LGN (Leu-Gly-Asn repeat enriched protein) that in turn interacts with the plasma membrane-anchored heterotrimeric protein $\mathrm{Ga}_{\mathrm{i}}$, thereby completing the connection between the microtubule and the plasma membrane (Figure 1.10) (Zheng et al., 2010). In interphase, NuMA resides in the nucleus and is not available for LGN binding. As this interaction is required for LGN to adopt an active conformation, it does not interact with plasma membrane-bound $\mathrm{Ga}_{\mathrm{i}}$ (Di Pietro et al., 2016). Upon NEBD in early mitosis, NuMA is released from the nucleus and binds to LGN that is than recruited to the cortex by $\mathrm{Ga}_{\mathrm{i}}$. As the LGN-NuMA-Gaicomplex is only located the lateral and not the apical cell cortex during mitosis, the bipolar spindle is oriented parallel to the substratum, thus ensuring proper positioning of the spindle. However, the LGN-NuMA complex was not only reported to be linked to the plasma membrane, but also to the actin cortex via LGN interaction with the actin binding protein Afadin (Carminati et al., 2016). Further connection between the plasma membrane and the actomyosin cortex is generated by the actin-membrane crosslinking ERM (ezrin-radixinmoesin) proteins. These have been shown to be crucial for cortical tension, as this was decreased in Moesin depleted cells due to myosin II mis-localization (Fehon et al., 2010). Moreover, ERM proteins control spindle orientation by regulating the LGN-NuMA-Ga $\alpha_{i}$ localization at the mitotic cortex (Machicoane et al., 2014).

\subsubsection{Function of the mitotic spindle in chromosome alignment and segregation}

Following assembly and positioning of the bipolar spindle, it now has to fulfil its main function: proper alignment and segregation of the duplicated sister chromatids. To facilitate this process, highly dynamic microtubules emanating from the centrosomal spindle poles undergo growth and shrinkage in various directions until they attach to kinetochores (Guo et al., 2013). This mechanism for chromosome alignment is known as "search and capture" (Kirschner \& Mitchison, 1986). However, not only the search and capture mechanism, but also spindle assembly mediated by the RanGTP-gradient and the augmin complex as described above, promote microtubule-kinetochore attachment and stabilization (Heald \& Khodjakov, 2015). Another important factor that contributes to a stable microtubule-kinetochore attachment is the Ndc80 complex, that resides at the outer kinetochore during mitosis. Subsequently after attachment, the minus-end directed motor protein dynein starts to pull the sister chromatid pair poleward, whereas the plus-end directed kinesin CENP-E promotes the opposite movement (Guo et al., 2013). When both sister chromatids are bound by opposing spindle poles, the pushing and pulling forces, generated by dynein, CENP-E 
and other factors, move them toward the spindle equator so that finally all of them are positioned at the metaphase plate. Only when the SAC is satisfied, the sister chromatids become separated and are pulled towards the spindle poles.

\subsection{Chromosomal Instability}

Only an error-free progression through mitosis ending with faithful chromosome segregation will result in the generation of two euploid daughter cells harboring the same genetic information. However, defects that occur during mitotic progression have been shown to frequently cause chromosome mis-segregation, a process that is termed as chromosomal instability (CIN) (Thompson et al., 2010). Due to chromosomal instability, cells become aneuploid, a state in which the chromosome number of a cell deviates from the modal number of the haploid set of chromosomes. Although CIN was originally only used to refer to the persistent high rate of whole chromosome mis-segregation, soon this term was also used to describe the frequent loss of chromosome fragments. In order to differentiate between these two phenomena, the terms whole chromosome instability (W-CIN) and structural chromosome instability (S-CIN) were introduced (Sansregret et al., 2018). In the following, the term chromosomal instability is used for W-CIN and the term structural chromosome instability is used separately. Both types of chromosomal instability result in an aberrant genomic state, that is the most common characteristic of solid tumors. Due to persistently high rates of chromosome mis-segregation, cells become aneuploid and acquire genetic heterogeneity (Heng et al., 2013). Although most chromosome missegregation events result in two daughter cells, that undergo apoptosis or that at least do not inherit an advantage through the gain or loss or chromosomes, there need to be just a small fraction of cells obtaining selective or adaptive advantage by the new chromosome combination. This in turn is the main driver for the development of cancer heterogeneity, which is the major cause of therapy resistance and poor patient's survival (Bach et al., 2019). Therefore, it is of utmost interest to investigate strategies, that suppress CIN and thus prevent therapy resistance. Especially colorectal cancer cells (CRC) have been found to exhibit genomic heterogeneity due to chromosome instability. About $80-85 \%$ of CRCs have the CIN phenotype associated with poor patient's prognosis, whereas with about $15 \%$ most non-CIN CRCs exhibit the microsatellite instability (MIN/MSI) phenotype. The MIN phenotype is characterized by mutations of genes participating in DNA mismatch repair and is associated with good patient's outcome (Cisyk et al., 2015; Dunican et al., 2002). 


\subsubsection{Mechanisms of whole chromosome instability}

Whole chromosome mis-segregation can appear through different mechanisms that compromise the SAC, sister chromatid cohesion, centrosome amplification or the stability of microtubulekinetochore attachments (Thompson et al., 2010). As described above, the SAC recognizes all kinds of erroneous attachments that are associates with the assembly of the MCC at unattached kinetochores and with weak kinetochore tension (Lara-Gonzalez et al., 2012). Correct chromosome segregation takes place when kinetochores of sister chromatids are attached to microtubules from opposing spindle poles in a bi-oriented (amphitelic) manner (Figure 1.12) (Godek et al., 2015). Then the MCC is disassembled from the kinetochore and pulling forces from both spindle poles generate enough kinetochore tension, thereby satisfying the SAC. Monotelic attachments occur when only the kinetochore of one of the sister chromatids is bound to microtubules from one spindle pole. Due to binding of the MCC complex to the unattached kinetochore, the SAC is active and allows further mitotic progression only after correction of the erroneous attachment. In addition, monotelic as well as syntelic attachments, in which the kinetochores of both sister chromatids are bound to microtubules from the same spindle pole, only transmit pulling forces from one spindle pole and not from opposing poles resulting in weak kinetochore tension. Tension-related error correction depends on several factors, of which Aurora B kinase is the major player (Funabiki, 2019). Aurora B accumulates at kinetochores with weak tension, where it phosphorylates kinetochore substrates such as Ndc80, thereby destabilizing the microtubule-kinetochore attachment. Afterwards, the kinetochore is free for binding to another microtubule. In case the microtubule emanates from the correct pole, kinetochore tension increases resulting in a stable microtubule-kinetochore attachment that satisfies the SAC. Another kind of erroneous attachments are merotelic attachments, in which one kinetochore of the chromatid sister pair is attached to microtubules emanating from one spindle pole and the other kinetochore to microtubules growing from both spindle poles (Godek et al., 2015). Merotelic attachments cannot be detected by the SAC, as both the disassembly of the MCC at kinetochores as well as increased kinetochore tension take place. Consequently, this misattachment can result in lagging chromosomes, which lead to the formation of two aneuploid daughter cells when pulled to the incorrect spindle pole. Therefore, mechanisms that cause the frequent occurrence of lagging chromosomes also contribute to chromosomal instability (Gregan et al., 2011). However, other mechanisms, in which Aurora B again plays a major role, exist to 
a

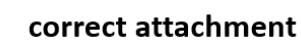

amphitelic

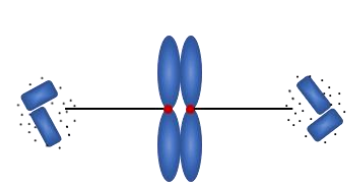

b

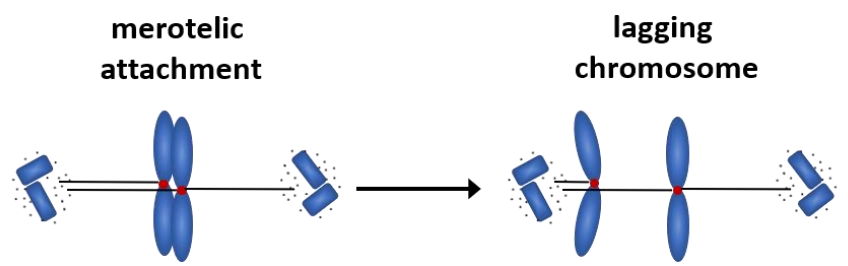

erroneous attachment

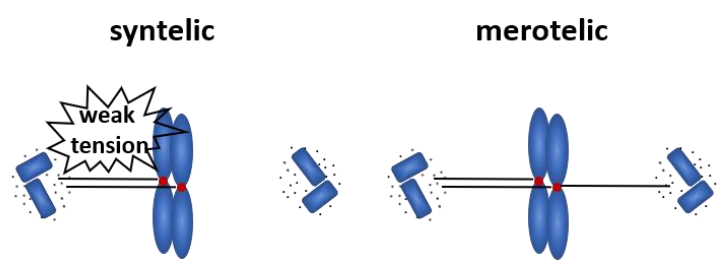

Figure 1.12: Modes of kinetochore-microtubule attachments. (a) In the correct, amphitelic kinetochoremicrotubule attachment, kinetochores of sister chromatids are attached to microtubules from opposing spindle poles in a bi-oriented manner. Monotelic attachments are formed when only the kinetochore of one of the sister chromatids is bound to microtubules from one spindle pole. This results in MCC complex binding to the unattached kinetochore and weak kinetochore tension, thereby activating the SAC. Syntelic attachments, in which the kinetochores of both sister chromatids are bound to microtubules from the same spindle pole, only transmit pulling forces from one spindle pole and not from opposing poles resulting in weak kinetochore tension and SAC activation. In merotelic attachments, one kinetochore of the chromatid sister pair is attached to microtubules emanating from one spindle pole and the other kinetochore to microtubules growing from both spindle poles. (b) Merotelic attachments do not activate the SAC. In case, chromosome segregation starts before the attachment is corrected, the sister chromatid that is attached to microtubules from one spindle pole is pulled toward this pole, while the other sister chromatid remains as lagging chromosome near the spindle equator. Later on, it is pulled to one of the two poles, resulting in chromosome mis-segregation when being pulled to the incorrect pole. Modified from Godek et al. (2015).

correct these attachments. In merotelic attachments, the attachment site of the incorrectly bound microtubule is positioned close to the region of the inner centromere, where Aurora $B$ is highly enriched (Cimini et al., 2006). Due to this spatial arrangement, Aurora B preferentially causes destabilization of microtubules emanating from the incorrect spindle pole. However, in case of an increased amount of merotelic attachments, these might not all be repaired in time, so that due to merotely-independent satisfaction of the SAC chromosome segregation is initiated even in the presence of these erroneous attachments. As especially the SAC and Aurora B are essential for proper chromosome segregation, a deregulated activity of these key players results in chromosome instability and aneuploidy, which has also been observed in several cancers (Huang et al., 2018; Minhas et al., 2003; Schuyler et al., 2012).

Another mechanism that has been shown to induce chromosome mis-segregation is an impaired sister chromatid cohesion (Thompson et al., 2010). A functional cohesion complex ensures proper sister chromatid connection, until it is cleaved at anaphase onset. Sister chromatids linked by 
improper cohesion, however, can also be separated upon erroneous attachments resulting in premature, incorrect chromosome segregation. Although only rarely observed, mutated genes involved in sister chromatid cohesion are associated with chromosomal instability and aneuploidy in cancer (Barber et al., 2008; Barbero, 2011; Sajesh et al., 2013).

Chromosome mis-segregation can also be induced by the presence of supernumary centrosomes (Thompson et al., 2010). These cells form multipolar spindles, that can lead to a cell division in more than two daughter cells, usually resulting in cell death (Ganem et al., 2009; Milunovic-Jevtic et al., 2016). However, some cells that harbor supernumary centrosomes form transient multipolar spindles in prometaphase, followed by clustering of the centrosomes into a bipolar spindle before chromosome segregation (Quintyne et al., 2005). This process drastically increases the number of merotelic attachments and thereby the amount of lagging chromosomes.

More recently, an increase in mitotic microtubule plus-end assembly rates was discovered as another mechanism for the induction of chromosomal instability (Ertych et al., 2014). Increased microtubule plus-end assembly rates were found to cause transient spindle positioning defects in prometaphase, that result in an increased rate of lagging chromosomes, chromosome missegregation and thus chromosome instability (Ertych et al., 2014; Silkworth \& Cimini, 2012).

\subsubsection{Mechanisms of structural chromosome instability}

Structural chromosome instability is associated with amplifications, deletions, inversions and translocations of chromosomal regions of various sizes (Lepage et al., 2019). It has been shown, that increased, unrepaired DNA double-strand breaks result in chromosomal breaks and thus in the formation of structural chromosome aberrations (W. F. Morgan et al., 1998; van Gent et al., 2001). DNA double strand breaks in turn, can be generated by several mechanisms, such as exogenous DNA-damaging agents or radiation, but also by endogenously generated replication stress. The latter one can be induced by oncogenic pathways that cause alterations of replication timing and progression and usually result in a slowing or stalling of the replication fork (Gaillard et al., 2015; Rickman \& Smogorzewska, 2019; Schoonen et al., 2019). The cellular response to replications stress contains the DNA-damage checkpoint, DNA repair or replication fork restart. If the cell fails to respond, the replication fork collapses followed by DNA double strand breaks (Cortez, 2015). The resulting chromosome fragments can undergo chromosome fusions and thereby form dicentric chromosomes and acentric chromosome fragments, such as so-called double minutes. Dicentric chromosomes are genetically unstable structures: when microtubules, which are bound to the two centromeres of one sister chromatid, pull to opposite poles, anaphase bridges are formed, followed by DNA breakage (Lopez et al., 2015). This process is part of so 
called breakage/fusion/bridge $(\mathrm{B} / \mathrm{F} / \mathrm{B})$ cycles and is known to produce chromosomal rearrangements, such as double minutes (Lo et al., 2002).

Interestingly, most tumors exhibit both whole and structural chromosome aberrations (Sansregret et al., 2018). Recent studies found that replication stress-induced structural chromosome abnormalities are drivers for whole chromosome mis-segregation in mitosis (Burrell et al., 2013). In turn, chromosome mis-segregation-associated lagging chromosomes have been shown to form micronuclei that are involved in the generation of DNA breaks (Crasta et al., 2012). These and other studies indicate, that there is a multidirectional correlation: key oncogenic pathways were identified as drivers for whole chromosome mis-segregation, that in turn is known to trigger structural chromosome aberrations, which can be activated by and activate itself oncogenic pathways (Bakhoum \& Swanton, 2014; Orr \& Compton, 2013). In this context, our group also found out, that mild replication stress is an inducer of increased mitotic microtubule plus-end assembly rates, thereby providing another link between structural and whole chromosome instability (Böhly et al., 2019).

\subsection{Aim of this study}

In the last decade cancer genome sequencing projects revealed a prevalence of diverse genetic heterogeneity in the vast majority of human cancers (Heng et al., 2013). Much of the genetic heterogeneity in tumors is due to chromosomal instability $(\mathrm{CIN})$, which was shown to contribute to tumorigenesis, tumor progression and therapy resistance (Holland \& Cleveland, 2012). This shows the importance of investigating the mechanisms leading to CIN in tumors. CIN is defined as an elevated rate of chromosome mis-segregation during mitosis and is, thus, resulting in the development of aneuploid cells (Thompson et al., 2010). The molecular mechanisms that are involved in causing CIN in human cancer cells are still poorly understood. However, some mechanisms have been proposed, such as a weakened spindle checkpoint signaling, the presence of supernumary centrosomes and defects in sister chromatid cohesion (Kops et al., 2005; Ganem et al., 2009; Barber et al., 2008). Recent work from our group provided another mechanistic insight into the generation of CIN by finding a link between CIN and increased microtubule plus-end assembly rates during mitosis (Ertych et al., 2014). These were further shown to be associated with spindle axis misalignment in prometaphase cells resulting in increased amounts of lagging chromosomes and thereby chromosome mis-segregation during anaphase. However, how increased microtubule plus-end assembly rates lead to spindle axis 
misalignment and, thus, CIN, in detail was still unknown. Recently, spindle positioning was found to be regulated by mitotic Arp2/3 dependent subcortical actin clouds that forward pulling forces generated from retraction fibers to centrosomes via binding to microtubules (Mitsushima et al., 2010; Kwon et al., 2015). At a similar time point, TRIO, a guanine exchange factor (GEF) for Rac1, was found to be part of the HET70 signature of genes that are most strongly upregulated in cancers with karyotype heterogeneity (Sheltzer, 2013), and also to contain an EB1-binding domain (van Haren et al., 2014). Knowing that EB1 is located at the growing microtubule plus-ends (Mimori-Kiyosue et al., 2000) and that Rac1 is a well-known Arp2/3 activator (H. N. Higgs \& Pollard, 1999) finally gave rise to a hypothetical signaling pathway between microtubule plus-end assembly rates and actin-regulated spindle positioning. Summed up, the putative pathway includes microtubule plus-tip-bound EB1, at which TRIO is bound and activates Rac1, that in turn acts as activator for the actin regulator Arp2/3. Recent work from our group supported the existence of such a pathway (Berger, 2016; Schermuly, 2019). However, the evidence for important links within this pathway were still missing, thereby necessitating further research. This especially included proof of the importance of the binding between EB1 and TRIO for this pathway and the microtubule plus-end assembly dependency of EB1-TRIO binding in mitotic cells. In this context, it was also tested whether TRIO binding to EB1 was necessary, or whether a higher TRIO and Rac1 activity independent from TRIO-EB1 binding was already sufficient for CIN development. Moreover, the actin cytoskeleton was analyzed more closely in order to figure out the missing link between Arp2/3 activity and spindle positioning. First results had not confirmed the involvement of this pathway in subcortical actin cloud formation, but rather showed an effect for the actin cortex, which has also been reported to affect spindle positioning through changes in the cortex structure and tension (Bovellan et al., 2014; Chugh et al., 2017; Kunda \& Baum, 2009). Therefore, further experiments for investigating a possible effect of the actin cortex structure and tension on spindle positioning were performed. 


\section{Material and Methods}

\subsection{Material}

Standard working materials including cell culture dishes, reaction tubes, pipette tips, filter tips, falcons and dishes for microscopy were purchased from Starlab (Hamburg, Germany), Sarstedt (Nümbrecht, Germany), Greiner BioOne (Frickenhausen, Germany) and ibidi (Martinsried, Germany).

\subsubsection{Equipment}

In Table 2.1. the equipment that was used for this study is listed.

Table 2.1 Equipment

\begin{tabular}{|c|c|c|}
\hline Equipment & Model & Company \\
\hline $\mathrm{CO}_{2}$ Incubator & $\begin{array}{l}\text { HERAcell } 240 \mathrm{CO}_{2} \\
\text { Incubator }\end{array}$ & $\begin{array}{l}\text { Thermo Fisher Scientific, Waltham, } \\
\text { MA, USA }\end{array}$ \\
\hline Centrifuge, cooling & Multifuge $X 3 R$ & $\begin{array}{l}\text { Thermo Fisher Scientific, Waltham, } \\
\text { MA, USA }\end{array}$ \\
\hline Centrifuge, tabletop & Biofuge pico & $\begin{array}{l}\text { Thermo Fisher Scientific, Waltham, } \\
\text { MA, USA }\end{array}$ \\
\hline Centrifuge, tabletop cooling & Biofuge fresco & $\begin{array}{l}\text { Thermo Fisher Scientific, Waltham, } \\
\text { MA, USA }\end{array}$ \\
\hline Chemiluminescence Imaging & Fusion-SL-3500.WL & Vilber Lourmat, Collégien, France \\
\hline Electroporation Device & GenePulser Xcell ${ }^{\odot}$ & $\begin{array}{l}\text { BioRad Laboratories, München, } \\
\text { Germany }\end{array}$ \\
\hline $\begin{array}{l}\text { Electrophoresis Power } \\
\text { Supply }\end{array}$ & Power Supply EV231 & Peqlab, Erlangen, Germany \\
\hline Flow Cytometer & BD FACSCanto ${ }^{\oplus} \|$ & $\begin{array}{l}\text { Becton Dickinson, San Jose, CA, } \\
\text { USA }\end{array}$ \\
\hline Thermomixer & $\begin{array}{l}\text { Thermomixer Comfort } \\
\mathrm{R}\end{array}$ & Eppendorf, Hamburg, Germany \\
\hline Heating Block & $\begin{array}{l}\text { TDB-120 Dry Block } \\
\text { Thermostat }\end{array}$ & Biosan, Riga, Latvia \\
\hline Laboratory Scale & $\begin{array}{l}\text { Sartorius Research } \\
\text { R200D }\end{array}$ & Sartorius, Göttingen, Germany \\
\hline Magnetic Mixer & IKAMAG ${ }^{\odot} \mathrm{RCT}$ & $\begin{array}{l}\text { IKA Labortechnik, Stauffen, } \\
\text { Germany }\end{array}$ \\
\hline \multirow{2}{*}{ Microscope } & Delta Vision Elite ${ }^{\odot}$ & $\begin{array}{l}\text { Applied Precision, Chalfont St. } \\
\text { Giles, UK }\end{array}$ \\
\hline & Leica DMI6000B & Leica, Wetzlar, Germany \\
\hline
\end{tabular}




\begin{tabular}{|c|c|c|}
\hline & Zeiss Axioscope FS & Zeiss, Oberkochen, Germany \\
\hline \multirow{3}{*}{ Microscope Camera } & sCMOS camera & $\begin{array}{l}\text { GE Healthcare, Chalfont St. Giles, } \\
\text { UK }\end{array}$ \\
\hline & Leica DFC369 FX & Leica, Wetzlar, Germany \\
\hline & $\begin{array}{l}\text { Hamamatsu ORCA-ER } \\
\text { C4742-95-12ER }\end{array}$ & $\begin{array}{l}\text { Hamamatsu Photonics, } \\
\text { Hamamatsu, Japan }\end{array}$ \\
\hline Microscope Camera Adaptor & A3474-07 & $\begin{array}{l}\text { Hamamatsu Photonics, } \\
\text { Hamamatsu, Japan }\end{array}$ \\
\hline Objective & Olympus $60 \times 1.40 \mathrm{NA}$ & Olympus, Tokyo, Japan \\
\hline Multilabel Reader & Victor $^{\odot}$ X3 & PerkinElmer, Rodgau, Germany \\
\hline Nitrocellulose Membrane & Protran BA 83 & $\begin{array}{l}\text { GE Healthcare, Chalfont St. Giles, } \\
\text { UK }\end{array}$ \\
\hline Pipettes & Pipetman $^{\odot}$ & $\begin{array}{l}\text { Gilson International, } \\
\text { LimburgOffheim, Germany }\end{array}$ \\
\hline Pipettor & Pipetboy acu & $\begin{array}{l}\text { Integra Biosciences, Fernwald, } \\
\text { Germany }\end{array}$ \\
\hline PVDF Membrane & ImmobilionR-P & $\begin{array}{l}\text { Merck Millipore, Darmstadt, } \\
\text { Germany }\end{array}$ \\
\hline Sterile Workbench & HERAsafeM & $\begin{array}{l}\text { Thermo Fisher Scientific, Waltham, } \\
\text { MA, USA }\end{array}$ \\
\hline Spectrophotometer & NanoDrop 2000 & $\begin{array}{l}\text { Thermo Fisher Scientific, Waltham, } \\
\text { MA, USA }\end{array}$ \\
\hline $\begin{array}{l}\text { Vertical Electrophoresis } \\
\text { System }\end{array}$ & & Own Manufacturing \\
\hline Vortex Mixer & VORTEX-GENIE ${ }^{\odot} 2$ & $\begin{array}{l}\text { Scientific Industries inc., Bohemia, } \\
\text { NY, USA }\end{array}$ \\
\hline Wet Blotting System & Mini Trans-Blot ${ }^{\circledR}$ Cell & $\begin{array}{l}\text { BioRad Laboratories, München, } \\
\text { Germany } \\
\text { Own Manufacturing }\end{array}$ \\
\hline
\end{tabular}




\subsubsection{Software}

In Table 2.2 the software that was used for data analysis in this study is listed.

Table 2.2 Software

\begin{tabular}{ll} 
Software & Company \\
\hline \hline Fiji Is Just ImageJ & $\begin{array}{l}\text { LOCI, University of Wisconsin-Madison, USA } \\
\text { GraphPad Software, San Diego, California, } \\
\text { USA }\end{array}$ \\
Hokawo Launcher 2.1 & Hamamatsu Photonics, Hamamatsu, Japan \\
Leica Application Suite 2.7.3.9723 & Leica, Wetzlar, Germany \\
softWoRx ${ }^{\odot}$ Explorer 1.3.0 & Applied Precision Inc., Issaquah, WA, USA \\
softWoRx ${ }^{\odot}$ 6.0 Software Suite & written and kindly provided by Prof. Dr. \\
CortexThickness & Burkhard Geil, Göttingen, Germany
\end{tabular}

\subsubsection{Chemicals}

All standard chemicals used in this study were purchased from Amersham Biosciences (Buckinghamshire, Great Britain), AppliChem (Darmstadt, Germany), Bio-techne (), BD Biosciences (Heidelberg, Germany), Carl Roth (Karlsruhe, Germany), Enzo Life Sciences (New York, NY, USA), Fermentas (St. Leon-Roth, Germany), Merck Millipore (Darmstadt, Germany), Promega (Madison, WI, USA), Roche Diagnostics (Mannheim, Germany), Santa Cruz (Dallas, Texas, USA), Sigma-Aldrich (St. Louis, Missouri, USA), Thermo Fisher Scientific (Waltham, MA, USA), Th. Geyer (Renningen, Germany) and VWR International (West Chester, PA, USA).

In Table 2.3 further chemicals as well as specific inhibitors are listed together with their functions and working concentrations. 
Table 2.3 Chemicals

\begin{tabular}{|c|c|c|c|}
\hline Chemical & $\begin{array}{l}\text { Working } \\
\text { Concentration }\end{array}$ & Effect & Company \\
\hline Blebbistatin (+/-) & $30 \mu \mathrm{M}$ & $\begin{array}{l}\text { Inhibition of the ATPase of the } \\
\text { NMII regulatory light chain }\end{array}$ & $\begin{array}{l}\text { Merck Millipore, } \\
\text { Darmstadt, } \\
\text { Germany }\end{array}$ \\
\hline CK666 & $20 \mu \mathrm{M}$ & $\begin{array}{l}\text { Inhibition of Arp2/3 dependent } \\
\text { actin assembly }\end{array}$ & $\begin{array}{l}\text { Merck Millipore, } \\
\text { Darmstadt, } \\
\text { Germany }\end{array}$ \\
\hline Dimethylenastron & $2 \mu \mathrm{M}$ & Inhibition of kinesin Eg5 & $\begin{array}{l}\text { Calbiochem, La } \\
\text { Jolla, CA, USA }\end{array}$ \\
\hline ITX3 & $15-50 \mu \mathrm{M}$ & $\begin{array}{l}\text { Inhibition of Trio N-Terminal } \\
\text { RhoGEF Domain }\end{array}$ & $\begin{array}{l}\text { Merck Millipore, } \\
\text { Darmstadt, } \\
\text { Germany }\end{array}$ \\
\hline Jasplakinolide & $100 \mathrm{nM}$ & $\begin{array}{l}\text { Promotion of actin } \\
\text { polymerization }\end{array}$ & $\begin{array}{l}\text { Santa Cruz, Dallas, } \\
\text { Texas, USA }\end{array}$ \\
\hline Latrunculin A & $0.75 \mu \mathrm{M}$ & $\begin{array}{l}\text { Inhibition of actin } \\
\text { polymerization }\end{array}$ & $\begin{array}{l}\text { Enzo Life } \\
\text { Sciences, New } \\
\text { York, USA }\end{array}$ \\
\hline $\begin{array}{l}\text { C3 toxin (Native } \\
\text { Clostridium } \\
\text { botulinum } \\
\text { exoenzyme) }\end{array}$ & $3 \mu \mathrm{g} / \mathrm{ml}$ & $\begin{array}{l}\text { Inhibition of Rho proteins by } \\
\text { ADP ribosylation }\end{array}$ & $\begin{array}{l}\text { Cytoskeleton Inc., } \\
\text { Denver, USA }\end{array}$ \\
\hline Nocodazole & $0.5 \mathrm{nM}$ & $\begin{array}{l}\text { Promotion of increased } \\
\text { microtubule plus-tip assembly } \\
\text { rate (at working concentration) }\end{array}$ & $\begin{array}{l}\text { Sigma-Aldrich, St. } \\
\text { Louis, Missouri, } \\
\text { USA }\end{array}$ \\
\hline NSC23766 & $40-100 \mu \mathrm{M}$ & $\begin{array}{l}\text { Inhibition of Rac1-GEF } \\
\text { interaction of TRIO and Tiam1 }\end{array}$ & $\begin{array}{l}\text { Santa Cruz, Dallas, } \\
\text { Texas, USA }\end{array}$ \\
\hline Calpeptin & $1 \mu \mathrm{g} / \mathrm{ml}$ & Activation of RhoA, B and C & $\begin{array}{l}\text { Santa Cruz, Dallas, } \\
\text { Texas, USA }\end{array}$ \\
\hline $\begin{array}{l}\text { AlexaFluor }{ }^{\mathrm{TM}} 594 \\
\text { Phalloidin }\end{array}$ & $100 \mathrm{nM}$ & $\begin{array}{l}\text { Fluorescent staining of F-actin } \\
\text { in fixed cells }\end{array}$ & $\begin{array}{l}\text { Thermo Fisher } \\
\text { Scientific, } \\
\text { Waltham, MA, USA }\end{array}$ \\
\hline SMIFH2 & $20 \mu \mathrm{M}$ & $\begin{array}{l}\text { Inhibitor of Formin } \mathrm{FH} 2 \\
\text { Domain, blocks actin } \\
\text { nucleation }\end{array}$ & $\begin{array}{l}\text { Merck Millipore, } \\
\text { Darmstadt, } \\
\text { Germany }\end{array}$ \\
\hline Taxol & $0.2-0.5 \mathrm{nM}$ & $\begin{array}{l}\text { Promotion of decreased } \\
\text { microtubule plus-tip assembly } \\
\text { rate (at working concentration) }\end{array}$ & $\begin{array}{l}\text { Sigma-Aldrich, St. } \\
\text { Louis, Missouri, } \\
\text { USA }\end{array}$ \\
\hline Thymidine & $2 \mathrm{mM}$ & $\begin{array}{l}\text { Synchronization of cells in } \\
\text { G1/S-Phase }\end{array}$ & $\begin{array}{l}\text { Sigma-Aldrich, St. } \\
\text { Louis, Missouri, } \\
\text { USA }\end{array}$ \\
\hline Y-27632 & $5-10 \mu \mathrm{M}$ & $\begin{array}{l}\text { Inhibitor of Rho-associated } \\
\text { kinase }\end{array}$ & $\begin{array}{l}\text { Absource } \\
\text { Diagnostics GmbH, } \\
\text { Munich, Germany }\end{array}$ \\
\hline
\end{tabular}




\subsubsection{Primary Antibodies}

In Table 2.4 all primary antibodies, their host species, clonality and the used dilution are listed.

Table 2.4 Primary Antibodies

\begin{tabular}{|c|c|c|c|c|c|}
\hline Antigen & $\begin{array}{l}\text { Host } \\
\text { species }\end{array}$ & Clonality & Use & Dilution & Company \\
\hline Arp3 (FMS338) & mouse & monoclonal & WB & $1: 500$ & $\begin{array}{l}\text { Sigma-Aldrich, St. } \\
\text { Louis, Missouri, USA }\end{array}$ \\
\hline$\alpha$-Tubulin (B-5-1-2) & mouse & monoclonal & $\begin{array}{l}\text { IF } \\
\text { WB }\end{array}$ & $\begin{array}{l}1: 700 \\
1: 2000\end{array}$ & $\begin{array}{l}\text { Santa Cruz, Dallas, } \\
\text { Texas, USA }\end{array}$ \\
\hline$\beta$-Actin (A5441) & mouse & monoclonal & WB & $1: 2000$ & $\begin{array}{l}\text { Sigma-Aldrich, St. } \\
\text { Louis, Missouri, USA }\end{array}$ \\
\hline ch-TOG $(\mathrm{H}-4)$ & mouse & monoclonal & WB & $1: 500$ & $\begin{array}{l}\text { Santa Cruz, Dallas, } \\
\text { Texas, USA }\end{array}$ \\
\hline EB1 (5) & mouse & monoclonal & $\begin{array}{l}\text { WB } \\
\text { IF }\end{array}$ & $\begin{array}{l}1: 500 \\
1: 100\end{array}$ & $\begin{array}{l}\text { BD Biosciences, } \\
\text { Heidelberg, Germany }\end{array}$ \\
\hline CENP-C & $\begin{array}{l}\text { guinea } \\
\text { pig }\end{array}$ & polyclonal & IF & $1: 1000$ & $\begin{array}{l}\text { MBL International } \\
\text { Corporation, Woburn, } \\
\text { USA }\end{array}$ \\
\hline ४-Tubulin (T3559) & rabbit & polyclonal & IF & $1: 700$ & $\begin{array}{l}\text { Sigma-Aldrich, St. } \\
\text { Louis, Missouri, USA }\end{array}$ \\
\hline y-Tubulin (T6557) & mouse & monoclonal & IF & $1: 1000$ & $\begin{array}{l}\text { Sigma-Aldrich, St. } \\
\text { Louis, Missouri, USA }\end{array}$ \\
\hline GFP (GF28R) & mouse & monoclonal & WB & $1: 3000$ & $\begin{array}{l}\text { Thermo Fisher } \\
\text { Scientific, Waltham, MA, } \\
\text { USA }\end{array}$ \\
\hline Op18 (E-3) & mouse & monoclonal & WB & $1: 300$ & $\begin{array}{l}\text { Santa Cruz, Dallas, } \\
\text { Texas, USA }\end{array}$ \\
\hline $\begin{array}{l}\text { Phospho-PAK1 } \\
\text { (Ser199/204)/ } \\
\text { PAK2 (Ser192/197) }\end{array}$ & rabbit & polyclonal & WB & $1: 1000$ & $\begin{array}{l}\text { Cell Signaling, } \\
\text { Cambridge; UK }\end{array}$ \\
\hline Rac1 [0.T.127] & mouse & monoclonal & WB & $1: 800$ & Abcam, Cambridge, UK \\
\hline TIAM1 & rabbit & polyclonal & WB & $1: 1000$ & $\begin{array}{l}\text { Bethyl Laboratories, } \\
\text { Montgomery, Texas, } \\
\text { USA }\end{array}$ \\
\hline TRIO & mouse & polyclonal & WB & $1: 500$ & $\begin{array}{l}\text { Abnova, Taipei City, } \\
\text { Taiwan }\end{array}$ \\
\hline TRIO (D-20) & goat & polyclonal & IF & $1: 500$ & $\begin{array}{l}\text { Santa Cruz, Dallas, } \\
\text { Texas, USA }\end{array}$ \\
\hline
\end{tabular}




\subsubsection{Secondary Antibodies}

In Table 2.5 all secondary antibodies, their host species, clonality and used dilution as well as the conjugated molecule are listed.

Table 2.5 Secondary Antibodies

\begin{tabular}{|c|c|c|c|c|c|c|}
\hline Antigen & Species & Clonality & Conjungate & Use & Dilution & Company \\
\hline $\begin{array}{l}\text { Anti-Guinea } \\
\text { Pig }\end{array}$ & goat & polyclonal & $\begin{array}{l}\text { Alexa- } \\
\text { Fluor594 }\end{array}$ & IF & $1: 1000$ & $\begin{array}{l}\text { Invitrogen, Carlsbad, } \\
\text { CA, USA }\end{array}$ \\
\hline Anti-Mouse & goat & polyclonal & $\begin{array}{l}\text { Alexa- } \\
\text { Fluor488 }\end{array}$ & IF & $1: 1000$ & $\begin{array}{l}\text { Invitrogen, Carlsbad, } \\
\text { CA, USA }\end{array}$ \\
\hline Anti-Rabbit & goat & polyclonal & $\begin{array}{l}\text { Alexa- } \\
\text { Fluor594 }\end{array}$ & IF & $1: 1000$ & $\begin{array}{l}\text { Invitrogen, Carlsbad, } \\
\text { CA, USA }\end{array}$ \\
\hline Anti-Mouse & goat & polyclonal & $\begin{array}{l}\text { Horseradish } \\
\text { Peroxidase } \\
\text { (HRP) }\end{array}$ & WB & $1: 10000$ & $\begin{array}{l}\text { Dianova, Hamburg, } \\
\text { Germany }\end{array}$ \\
\hline Anti-Rabbit & goat & polyclonal & $\begin{array}{l}\text { Horseradish } \\
\text { Peroxidase } \\
\text { (HRP) }\end{array}$ & WB & $1: 10000$ & $\begin{array}{l}\text { Dianova, Hamburg, } \\
\text { Germany }\end{array}$ \\
\hline
\end{tabular}

\subsubsection{Oligonucleotides}

In Table 2.6 all used siRNAs, their sequences and references are listed.

Table 2.6 siRNAs

\begin{tabular}{|c|c|c|}
\hline Target Gene & Sequence & Reference \\
\hline \multirow{2}{*}{ Arp3 } & 5'-AGGUUUAUGGAGCAAGUGA-3' & \multirow{2}{*}{ Steffen et al. 2006} \\
\hline & 5'-GCCAAAACCUAUUGAUGUA-3' & \\
\hline CKAP5 & 5'-GAGCCCAGAGTGGTCCAAA-3' & De Luca et al. 2008 \\
\hline EB1 & $5^{\prime}$-AUUCCAAGCUAAGCUAGAA-3' & $\begin{array}{l}\text { Watson \& Stephens } \\
2006\end{array}$ \\
\hline LUCIFERASE & 5'-CUUACGCUGAGUACUUCGAUU-3' & Elbashir et al. 2001 \\
\hline Rac1 & 5'-AAGGAGATTGGTGCTGTAAAA-3' & Chan et al. 2005 \\
\hline \multirow{2}{*}{ TRIO } & 5'-GAUAAGAGGUACAGAGAUU-3' & \multirow{2}{*}{ Cannet et al. 2014} \\
\hline & 5'-GGAAGUCGCUCCUUGACAA-3' & \\
\hline
\end{tabular}




\subsubsection{Plasmids}

In Table 2.7 all used plasmids, their purpose and reference are listed.

Table 2.7 Plasmids

\begin{tabular}{|c|c|c|}
\hline Vector & Purpose & Reference \\
\hline pBluescript KS+ & $\begin{array}{l}\text { Cloning vector, used as carrier DNA } \\
\text { for calcium phosphate transfection in } \\
\text { this study }\end{array}$ & $\begin{array}{l}\text { Kindly provided by Prof. Heike } \\
\text { Krebber, Göttingen, Germany }\end{array}$ \\
\hline pcDNA3.1 & $\begin{array}{l}\text { CMV-promotor driven expression } \\
\text { vector for human cells }\end{array}$ & Invitrogen, Carlsbad, CA, USA \\
\hline pcDNA-RFP-H2B & $\begin{array}{l}\text { CMV-promoter driven expression of } \\
\text { RFP-tagged } H 2 B \text { in human cells }\end{array}$ & $\begin{array}{l}\text { Kindly provided by Prof. Ody } \\
\text { Sibon, Groningen, The } \\
\text { Netherlands }\end{array}$ \\
\hline $\begin{array}{l}\text { PcDNA3-TIAM1- } \\
\text { C1199 }\end{array}$ & $\begin{array}{l}\text { CMV-promoter driven expression of a } \\
\mathrm{N} \text {-terminal truncated version (aa 393- } \\
\text { 1591) of TIAM1 }\end{array}$ & $\begin{array}{l}\text { Kindly provided by Prof. } \\
\text { Angeliki Malliri, Manchester, } \\
\text { UK }\end{array}$ \\
\hline $\begin{array}{l}\text { pCS2-EGFP- } \\
\text { hUtrophinCH }\end{array}$ & $\begin{array}{l}\text { CMV-promoter driven expression of } \\
\text { the GFP-tagged Calponin homology } \\
\text { domain of human Utrophin (aa 1-261) } \\
\text { to detect F-actin in mammalian cells }\end{array}$ & $\begin{array}{l}\text { Kindly provided by Dr. Melina } \\
\text { Schuh, Göttingen, Germany }\end{array}$ \\
\hline pEGFP-C1 & $\begin{array}{l}\text { CMV-promotor driven GFP- } \\
\text { expressing vector for human cells }\end{array}$ & $\begin{array}{l}\text { Clontech, Saint-Germain-en- } \\
\text { Laye, France }\end{array}$ \\
\hline pEGFP-H2B & $\begin{array}{l}\text { CMV-promoter driven expression of } \\
\text { GFP-tagged } H 2 B \text { in human cells }\end{array}$ & $\begin{array}{l}\text { Kindly provided by Prof. Stefan } \\
\text { Gaubatz, Marburg, Germany }\end{array}$ \\
\hline pEGFP-EB3 & $\begin{array}{l}\text { CMV-promotor driven expression of } \\
\text { GFP-tagged EB3 in human cells }\end{array}$ & $\begin{array}{l}\text { Kindly provided by Prof. Linda } \\
\text { Wordeman (Seattle, WA, USA) }\end{array}$ \\
\hline pmCherry-EB3 & $\begin{array}{l}\text { CMV-promotor driven expression of } \\
\text { mCherry-tagged } E B 3 \text { in human cells }\end{array}$ & $\begin{array}{l}\text { Kindly provided by Prof. Linda } \\
\text { Wordeman (Seattle, WA, USA) }\end{array}$ \\
\hline $\begin{array}{l}\text { pEGFP-Rac1 } \\
\text { Q61L }\end{array}$ & $\begin{array}{l}\text { CMV-promotor driven expression of } \\
\text { GFP-tagged constitutively active } \\
\text { Rac1 in human cells }\end{array}$ & $\begin{array}{l}\text { Kindly provided by Prof. } \\
\text { Robert Grosse (Marburg, } \\
\text { Germany) }\end{array}$ \\
\hline pEGFP-TRIO & $\begin{array}{l}\text { CMV-promotor driven expression of } \\
\text { GFP-tagged TRIO in human cells }\end{array}$ & $\begin{array}{l}\text { Kindly provided by Anne } \\
\text { Debant (Montpellier, France) }\end{array}$ \\
\hline $\begin{array}{l}\text { pEGFP-TRIO- } \\
\text { SRNN }\end{array}$ & $\begin{array}{l}\text { CMV-promotor driven expression of } \\
\text { GFP-tagged TRIO in human cells }\end{array}$ & $\begin{array}{l}\text { Kindly provided by Anne } \\
\text { Debant (Montpellier, France) }\end{array}$ \\
\hline pmCherry-EB1 & $\begin{array}{l}\text { CMV-promotor driven expression of } \\
\text { mCherry-tagged } E B 1 \text { in human cells }\end{array}$ & $\begin{array}{l}\text { Addgene, Michael Davidson } \\
\text { Lab }\end{array}$ \\
\hline
\end{tabular}




\subsubsection{Human Cell Lines}

In Table 2.8 all human cell lines, their origin and reference are listed.

Table 2.8 Human Cell Lines

\begin{tabular}{|c|c|c|}
\hline Cell Line & Origin & Reference \\
\hline HCT116 & colon carcinoma & obtained from ATCC, Manassas, Virginia, USA \\
\hline HEK293T & embryonic kidney & obtained from ATCC, Manassas, Virginia, USA \\
\hline HT29 & colon carcinoma & obtained from ATCC, Manassas, Virginia, USA \\
\hline MCF10A & mammary gland & $\begin{array}{l}\text { obtained from Horizon Discovery, Cambridge; } \\
\text { UK }\end{array}$ \\
\hline $\begin{array}{l}\text { MCF10A } \\
\text { Rac1Q61L +/- }\end{array}$ & mammary gland & $\begin{array}{l}\text { obtained from Horizon Discovery, Cambridge; } \\
\text { UK }\end{array}$ \\
\hline SW620 & colon carcinoma & obtained from ATCC, Manassas, Virginia, USA \\
\hline SW480 & colon carcinoma & obtained from ATCC, Manassas, Virginia, USA \\
\hline
\end{tabular}

In Table 2.9 human cell lines that were generated in this study are listed.

Table 2.9 Generated Human Cell Lines

\begin{tabular}{llll} 
Cell Line & $\begin{array}{l}\text { Parental Cell } \\
\text { Line }\end{array}$ & Plasmid & Selection Marker \\
\hline \hline HCT116-GFP & HCT116 & pEGFP-C1 & Geneticin \\
$\begin{array}{l}\text { HCT116-TRIO-GFP } \\
\text { HCT116-TRIO-SRNN- } \\
\text { GFP }\end{array}$ & HCT116 & pEGFP-TRIO & Geneticin \\
\hline HCT116 & pEGFP-TRIO-SRNN & Geneticin
\end{tabular}

\subsection{Cell Biological Methods}

\subsubsection{Cultivation of Human Cell Lines}

HCT116, HT29, SW620 and SW480 cells were cultured in RPMI1640 (PAN-Biotech GmbH, Aidenbach, Germany) supplemented with 10\% FCS (Gibco ${ }^{\mathrm{TM}}$ by Thermo Fisher Scientific, Waltham, MA, USA), $100 \mu \mathrm{g} / \mathrm{ml}$ streptomycin and $100 \mathrm{units} / \mathrm{ml}$ penicillin (PAN-Biotech $\mathrm{GmbH}$, Aidenbach, Germany) at $37^{\circ} \mathrm{C}$ and $5 \% \mathrm{CO}_{2}$. HEK293T cells were cultured in DMEM (PANBiotech $\mathrm{GmbH}$, Aidenbach, Germany) containing 10\% FCS, $100 \mu \mathrm{g} / \mathrm{ml}$ streptomycin and 
100 units/ml penicillin. For cultivation of MCF10A and MCF10A Rac1Q61L+/- cells DMEM/F12 (PAN-Biotech $\mathrm{GmbH}$, Aidenbach, Germany) was used and had been supplemented with 5\% horse serum (Gibco ${ }^{\text {TM }}$ by Thermo Fisher Scientific, Waltham, MA, USA), $100 \mu \mathrm{g} / \mathrm{ml}$ streptomycin and 100 units/ml penicillin, 20 ng/ml EGF (PeproTech, Hamburg, Germany), 0.5 g/ml hydrocortisone (Sigma-Aldrich, St. Louis, Missouri, USA), $100 \mathrm{ng} / \mathrm{ml}$ Cholera toxin (Sigma-Aldrich, St. Louis, Missouri, USA) and $10 \mu \mathrm{g} / \mathrm{ml}$ insulin (Sigma-Aldrich, St. Louis, Missouri, USA). The cells were passaged every two to three days. For this purpose, they were washed once with PBS and detached by using trypsin/EDTA (Lonza Group Ltd., Basel, Switzerland). Depending on the growth rate of the used cell line and its confluency at the day of passage various amounts of cell suspension were transferred to a new cell culture dish containing fresh medium. For long-term storage cells were harvested and resuspended in freezing medium (cell line specific medium with supplements and additional 10\% FCS (v/v) and 10\% (v/v) DMSO). Afterwards they were cooled down gently to $-80^{\circ} \mathrm{C}$ using an isopropanol-based cryo $1^{\circ} \mathrm{C}$ freezing container and after at least $24 \mathrm{~h}$ they were transferred to liquid nitrogen.

\subsubsection{Transfection of human cells}

\subsubsection{1 siRNA transfection}

siRNA transfections were performed by using either INTERFERin ${ }^{\circledR}$ (Polyplus, Illkirch, France) or ScreenFect ${ }^{\circledR}$ (ScreenFect GmbH, Eggenstein-Leopoldshafen, Germany). Before transfection cells were seeded with a confluency of 70-80\% in 6-well plates and were transfected after they had become attached again to up to $24 \mathrm{~h}$ after seeding. $48 \mathrm{~h}$ after transfection cells were used for experiments and knock down efficiency was checked by performing Western blots of cell lysates.

\section{INTERFERin® Transfection}

After 60 pmol siRNA were diluted in $188 \mu$ l serum free medium, $6 \mu$ INTERFERin ${ }^{\circledR}$ were added and the mixture was vortexed for $10 \mathrm{~s}$. During 10 min of incubation at RT, cells that have been seeded for transfection were washed with PBS and $1 \mathrm{ml}$ fresh medium was added to the cells. Then the siRNA mixture was dropped onto the cells. After incubation for $4 \mathrm{~h}$ at $37^{\circ} \mathrm{C}$ and $5 \%$ $\mathrm{CO}_{2}$, medium was changed.

\section{ScreenFect ${ }^{\circledR}$ siRNA Transfection}

Before use ScreenFect ${ }^{R} \operatorname{siRNA}$ transfection reagent was vortexed for $10 \mathrm{~s} .4 \mu \mathrm{l}$ of ScreenFect ${ }^{\circledR}$ siRNA transfection reagent were added to $30 \mu$ of ScreenFect ${ }^{\circledR}$ dilution buffer and 
the mixture was vortexed for $3 \mathrm{~s}$. In another tube 40 pmol siRNA were diluted in $30 \mu \mathrm{l}$ of ScreenFect ${ }^{\circledR}$ dilution buffer and the mixture was also vortexed for $3 \mathrm{~s}$. Afterwards, the diluted ScreenFect ${ }^{\circledR}$ SiRNA transfection reagent was transferred to the diluted SiRNA and mixed by pipetting 10 times up and down. During an incubation of $15 \mathrm{~min}$ at RT, medium of cells was changed to $1.5 \mathrm{ml}$ antibiotic free medium. The transfection mixture was added drop-wise onto the cells which then were incubated at $37^{\circ} \mathrm{C}$ at $5 \% \mathrm{CO}_{2}$. Next day, the medium was changed.

\subsubsection{Plasmid Transfection}

Depending on the plasmid size and the purpose, plasmid transfection was performed by the following transfection methods. Independent of the transfection method, cells were used for experiments and transfection efficiency was checked by fluorescence microscopy or by performing Western blots of cell lysates $48 \mathrm{~h}$ after transfection.

\section{Electroporation}

Transfection of plasmids by electroporation was performed for most of the used cell lines and plasmids of small to medium size (up to $8 \mathrm{~kb}$ ). Cells were trypsinated and counted. Then they were centrifuged for $5 \mathrm{~min}$ and $1500 \mathrm{rpm}$ and resuspended in fresh medium to a concentration of $2.5 \times 10^{6} \mathrm{cells} / \mathrm{ml}$. 10-20 $\mu \mathrm{g}$ of plasmid DNA was mixed with $400 \mu \mathrm{l}$ of cell suspension and transferred to a $4 \mathrm{~mm}$ cuvette. HCT116, SW620, SW480 and HEK293T cells were electroporated using a electroporator (BioRad, Hercules, California, USA) at $300 \mathrm{~V}$ and $500 \mu \mathrm{F}$. Cells were gently transferred into 6 -well plates containing fresh medium and incubated at $37^{\circ} \mathrm{C}$ and $5 \% \mathrm{CO}_{2}$. After $4 \mathrm{~h}$, medium was changed.

\section{Plasmid transfection using Calcium Phosphate}

For transfection of HEK293T cells, they were seeded in $10 \mathrm{~cm}$ dishes to be $60-70 \%$ confluent the next day. At the day of transfection, $10 \mu \mathrm{g}$ target DNA and $10 \mu \mathrm{g}$ carrier DNA, for which pBluescript was used, as well as $62 \mu \mathrm{l} 2.5 \mathrm{M} \mathrm{CaCl}_{2}$ were diluted with water to a total volume of $500 \mu \mathrm{l}$ and mixed by carefully pipetting up and down. By using a pipette controller with a sterile plugged Pasteur pipette air was blown into a $500 \mu \mathrm{l}$ 2x HBS (280 mM NaCl, $50 \mathrm{nmM}$ HEPES, $1.5 \mathrm{mM}$ $\mathrm{Na}_{2} \mathrm{HPO}_{4}, \mathrm{pH}$ 7.05) containing falcon. Subsequently, the $500 \mu \mathrm{l}$ DNA mixture was added to the $2 x$ HBS solution drop-wise and air was blown into the mixture for further $30 \mathrm{~s}$. Then the mixture was added drop-wise onto the cells an incubated overnight at $37^{\circ} \mathrm{C}$ and $5 \% \mathrm{CO}_{2}$. The following day, cells were washed twice with PBS and fresh medium was added. 


\section{Plasmid transfection using ScreenFect ${ }^{\circledR} A$}

Cells were seeded with a confluency of 70-80 \% in 6-well plates and were transfected after they had become attached again to up to $24 \mathrm{~h}$ after seeding. Before use ScreenFect $\circledast \mathrm{A}$ transfection reagent (ScreenFect GmbH, Eggenstein-Leopoldshafen, Germany) was vortexed for $10 \mathrm{~s} .6 \mu \mathrm{l}$ of ScreenFect ${ }^{\circledR} A$ transfection reagent were added to $30 \mu$ of ScreenFect ${ }^{\circledR}$ dilution buffer and the mixture was vortexed for $3 \mathrm{~s}$. In another tube, 0.25-1.5 $\mu$ g plasmid DNA were diluted in $30 \mu \mathrm{l}$ of ScreenFect ${ }^{\circledR}$ dilution buffer and the mixture was also vortexed for $3 \mathrm{~s}$. Afterwards, the diluted ScreenFect ${ }^{\circledR}$ siRNA transfection reagent was transferred to the diluted plasmid DNA and mixed by pipetting 10 times up and down, followed by 15 min incubation at RT. In the meantime, medium of cells were changed to $1.5 \mathrm{ml}$ fresh medium without antibiotics. The transfection mixture was added drop-wise onto the cells which then were incubated at $37^{\circ} \mathrm{C}$ at $5 \% \mathrm{CO}_{2}$. The following day, the medium was changed.

\section{Plasmid transfection using Lipofectamine ${ }^{\mathrm{TM}} 3000$ Transfection Reagent}

Lipofectamine $^{\mathrm{TM}} 3000$ (Invitrogen $^{\mathrm{TM}}$ by Thermo Fisher Scientific, Waltham, MA, USA) transfection was used for large plasmids. One day before transfection, cells were seeded in 6-well plates to yield a confluency of $70-80 \%$ at the day of transfection. For transfection, $125 \mu \mathrm{l} \mathrm{serum}$-free medium was mixed with $6.25 \mu$ of Lipofectamine ${ }^{\text {TM }} 3000$ Transfection Reagent by vortexing for $3 \mathrm{~s}$. In another tube $2.5 \mu \mathrm{g}$ plasmid DNA and $5 \mu \mathrm{l}$ of $\mathrm{P}^{3000^{\text {TM }}}$ Reagent was added to $125 \mu \mathrm{l}$ serum-free

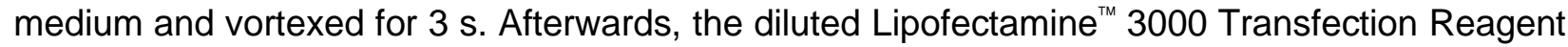
was transferred to the plasmid DNA and $\mathrm{P} 3000^{\mathrm{Tm}}$ Reagent containing solution and mixed by pipetting 10 times up and down, followed by 15 min incubation at RT. Meanwhile, medium of cells were changed to $2 \mathrm{ml}$ fresh medium without antibiotics. The transfection mixture was added dropwise onto the cells which then were incubated at $37^{\circ} \mathrm{C}$ at $5 \% \mathrm{CO}_{2}$. The following day, the medium was changed.

\subsubsection{Generation of stable cell lines}

To generate HCT116 cell lines stably expressing EGFP, TRIO-WT-GFP or TRIO-SRNN-GFP, cells were transfected using Lipofectamine ${ }^{\mathrm{TM}} 3000$ as described above. For selection of stably transfected cells, medium was changed to medium containing $300 \mu \mathrm{g} / \mathrm{ml} \mathrm{G} 418$ (Sigma-Aldrich, St. Louis, Missouri, USA) one day after transfection. After three more days, serially diluted cell suspensions were seeded in $10 \mathrm{~cm}$ dishes and GFP-positive single cell clones were isolated for further cultivation and analysis 7-10 days later. 


\subsubsection{Karyotype Analysis via Chromosome Spreading}

To accumulate cells in mitosis, cells that have been seeded in 6-well plates were treated with $2 \mu \mathrm{M}$ DME for 4-5 h. Afterwards, supernatant medium was spun down at $2000 \mathrm{rpm}$ for 5 min together with residual cells that have been detached by treatment with $0.5 \mathrm{mM}$ EDTA/PBS. Cells were resuspended in $750 \mu \mathrm{l}$ hypotonic solution (10\% cell line specific medium without supplements, e.g. RPMI, DMEM, DMEM/F12, $60 \%$ aqua dest.) and incubated for $15 \mathrm{~min}$ at RT. Afterwards, $250 \mu$ ice-cold Carnoy's fixative (75 \% methanol, $25 \%$ glacial acetic acid) was added and cells were centrifuged at 2000 rpm for $5 \mathrm{~min}$. Cells were resuspended in $1 \mathrm{ml}$ ice-cold Carnoy's fixative and centrifuged again. Having repeated the last fixation step one more time, the cell suspension was incubated for at least $15 \mathrm{~min}$ on ice or stored at $-20^{\circ} \mathrm{C}$ until further procedure. For spreading, cells were resuspended in 300-500 $\mu \mathrm{l}$ glacial acetic acid and dropped from approximate $50 \mathrm{~cm}$ height onto an ethanol-washed, pre-cooled, wet object slide. After 5 min incubation on a $42^{\circ} \mathrm{C}$ heat block wrapped with wet paper towels, slides were air-dried. For staining, slides were transferred into a 8 \% Giemsa solution (Sigma-Aldrich, St. Louis, Missouri, USA) for 15 min. Afterwards, slides were washed with water and air-dried at RT, followed by being embedded in Euparal (Carl Roth, Karlsruhe, Germany). For analysis of chromosome spreads, Zeiss Axioscope FS microscope equipped with a Hamamatsu C4742-95-12ER camera and the Hokawo Launcher 2.1 software was used.

\subsubsection{Synchronization of human cells}

\section{Synchronization of cells via double thymidine block}

To synchronize cell cycle progression of human cell lines, a double thymidine block which arrests cells at G1/S phase was performed. For this purpose, cells were grown in $2 \mathrm{mM}$ thymidine containing cell culture medium for $16 \mathrm{~h}$ and subsequently washed six times for 5 min with fresh medium. After washing, cells were grown in cell culture medium for further $7.5 \mathrm{~h}$ and then seeded onto glass coverslips in $2 \mathrm{mM}$ thymidine containing medium for another $16 \mathrm{~h}$. Afterwards, cells were washed and released in fresh medium as described above. In order to analyze lagging chromosomes, cells accumulated at anaphase stage were fixed. The highest accumulation of anaphase cells was $8.0 \mathrm{~h}$ after washing for HCT116 cells and $10.5 \mathrm{~h}$ after washing for MCF10A cells. 


\subsubsection{Immunofluorescence microscopy}

Method-specific immunofluorescence staining is described in the method-related chapters following below.

For immunofluorescence staining of EB1 and TRIO, cells grown on glass coverslips were fixed with ice-cold methanol for 6 min at $-20^{\circ} \mathrm{C}$, followed by washing twice with PBS and blocking with $5 \%$ FCS/PBS for $30 \mathrm{~min}$. Staining was carried out by incubating cells with goat anti-TRIO (1:500) and mouse anti-EB1 (1:100) antibodies diluted in $2 \%$ FCS/PBS for 90 min at RT. Afterwards, cells were washed three times with PBS, followed by an incubation with host-specific fluorescencelabeled secondary antibodies (1:1000 dilution in $2 \%$ FCS/PBS) for $90 \mathrm{~min}$ at RT. DNA was stained with Hoechst33342 (1:15,000 in PBS; Sigma-Aldrich, St. Louis, Missouri, USA) for 5 min at RT. Afterwards, cells were washed four times with PBS and one time with Millipore water. Coverslips were air-dried and embedded using VectaShield ${ }^{\circledR}$ (Vector Laboratories, Burlingame, CA, USA). To analyze fixed and stained cells, a Delta Vision Elite ${ }^{\odot}$ microscope equipped with a PCO Edge sCMOS camera and an Olympus 60x 1.40 NA objective was used. Images were acquired with a z-optical spacing of $0.2 \mu \mathrm{m}$ and deconvolved using the softWoRx ${ }^{\circledR} 6.0$ software.

\subsubsection{Analysis of spindle axis alignment of prometaphase cells}

For the analysis of spindle axis alignment of cells in prometaphase, asynchronously growing cells were seeded on fibronectin-coated coverslips. Next day, cells were and fixed with ice-cold methanol for 6 min at $-20^{\circ} \mathrm{C}$. Subsequently, cells were washed twice with PBS and blocked with $10 \%$ FCS/PBS for 30 min at RT. For staining of centrosomes and microtubules, cells were incubated with rabbit anti- $\gamma$-Tubulin $(1: 1000)$ and mouse anti- $\alpha$-Tubulin $(1: 700)$ diluted in $2 \%$ FCS/PBS for 90 min at RT. Cells were washed three times with PBS and incubated with hostspecific fluorescence-labeled secondary antibodies (1:1000 dilution in $2 \%$ FCS/PBS) for 90 min at RT. DNA staining was carried out using Hoechst33342 (1:15,000 in PBS) for 5 min at RT. After washing the cells four times with PBS and once with Millipore water, coverslips were air-dried and embedded using VectaShield ${ }^{\circledR}$. Fixed and stained cells were analyzed using a Delta Vision Elite ${ }^{\odot}$ microscope equipped with a PCO Edge sCMOS camera and an Olympus 60x 1.40 NA objective. Images were acquired with a z-optical spacing of $0.2 \mu \mathrm{m}$ and deconvolved using the softWoRx ${ }^{\circledR}$ 6.0 software. The angle of spindle axis alignment was determined by using the softWoRx ${ }^{\circledR}$ Explorer 1.3.0 and the following formula:

$$
\alpha(\text { spindle axis angle })=\arcsin \left(\frac{\text { number of z-stacks between centrosomes } x 0.4 \mu \mathrm{m}}{\text { distance between centrosomes }[\mu \mathrm{m}]}\right) * \frac{180^{\circ}}{\pi}
$$




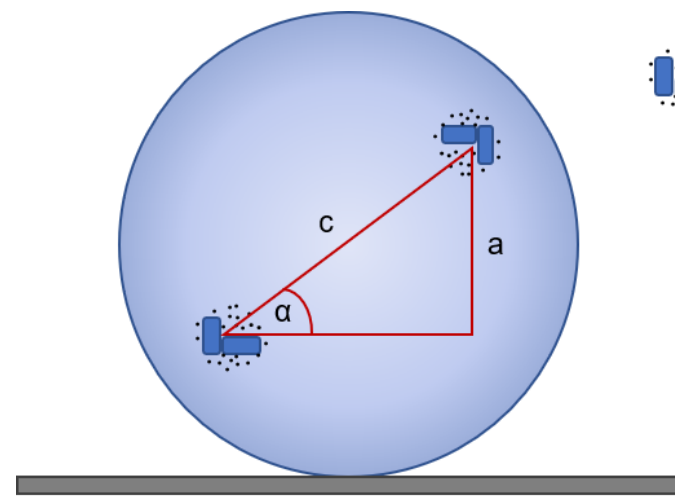

centrosome

$\mathrm{a}=$ height in $\mu \mathrm{m}$

(number of Z-stacks between

centrosomes $\times 0.4 \mu \mathrm{m}$ )

$\mathrm{c}=$ distance between centrosomes in $\mu \mathrm{m}$

$\alpha=$ spindle axis angle

$\alpha=\arcsin \left(\frac{a}{c}\right) * \frac{180^{\circ}}{\pi}$

substratum

Figure 2.1: Schematic illustration for the calculation of the spindle axis angle.

\subsubsection{Analysis of lagging chromosomes}

In order to stain centromeres and microtubules for the analysis of lagging chromosomes, cells grown on glass coverslips have been synchronized by a double thymidine block and were fixed with $2 \%$ PFA for 5 min at RT when accumulated in anaphase. Subsequently, cells underwent further fixation with ice-cold methanol for $5 \mathrm{~min}$ at $-20^{\circ} \mathrm{C}$. Afterwards, cells were washed twice with PBS and blocked with $5 \%$ FCS/PBS for 30 min. Staining of centromeres and microtubules was performed by incubating cells with guinea pig anti-CENP-C (1:1000) and mouse anti- $\alpha-T u b u l i n$ (1:700) antibodies diluted in $2 \%$ FCS/PBS for 90 min at RT. After washing the cells three times with PBS, they were incubated with host-specific fluorescence-labeled secondary antibodies (1:1000 dilution in $2 \%$ FCS/PBS) for $90 \mathrm{~min}$ at RT. DNA was stained with Hoechst33342 (1:15,000 in PBS) for 5 min at RT. Afterwards, cells were washed four times with PBS and one time with Millipore water. Coverslips were air-dried and embedded using VectaShield ${ }^{\circledR}$. Microscopy of fixed and stained cells was performed using a Leica DM600B fluorescence microscope and an ORCA-ER camera or a Delta Vision Elite ${ }^{\odot}$ microscope equipped with a PCO Edge sCMOS camera. Image acquirement was carried out with a z-optical spacing of $0.4 \mu \mathrm{m}$, followed by deconvolution using the softWoRx ${ }^{\circledR} 6.0$ software. Lagging chromosomes were defined as Hoechst-positive and CENP-C-positive chromosomes located clearly separated in between the two pole-oriented chromosome masses in anaphase cells. Per experiment, the amount of lagging chromosomes of 100 anaphase cells was counted. 


\subsubsection{Analysis of microtubule plus-end assembly rates}

For the analysis of microtubule plus-end assembly rates, cells were transfected with $10 \mu \mathrm{g}$ pEGFP-EB3 or $10 \mu \mathrm{g}$ pmCherry-EB3 plasmid DNA via electroporation. Next day, cells were seeded into $\mu$-Slide 8 well chambers (ibidi, Martinsried, Germany). $48 \mathrm{~h}$ after transfection, cells were washed once with PBS and phenol red-free cell culture medium containing $2 \mu \mathrm{M}$ DME to arrest cells in mitosis was added. After incubation for 1-2 h, live cell microscopy of microtubule plus-end assembly rates was carried out at $37^{\circ} \mathrm{C}$ and $5 \% \mathrm{CO}_{2}$ using a Delta Vision Elite ${ }^{\odot}$ microscope equipped with a PCO Edge sCMOS camera and an environmental chamber for temperature control and $\mathrm{CO}_{2}$ injection. Images of 4 z-stacks with a z-optical spacing of $0.4 \mu \mathrm{m}$ were acquired every two seconds for a duration of $30 \mathrm{~s}$. Afterwards, images were deconvolved and the 4 z-stack images converted to $2 \mathrm{D}$ by using the softWoRx ${ }^{\circledR} 6.0$ software. Microtubule plusend assembly rates were determined by measuring the growth of microtubule plus-ends between two frames using the softWoRx ${ }^{\circledR}$ Explorer 1.3.0. Per experiment, microtubule plus-end assembly rates of 20 microtubules per cell and a total of 10 cells were measured.

\subsubsection{In vitro migration assay}

For the analysis of the migrative behavior of cells, in vitro migration assays were performed. Therefore, cells were starved for $16 \mathrm{~h}$ in cell culture medium with a reduced FCS content of $0.5 \%$. Afterwards, cells were trypsinized, counted and resuspended in medium containing $0.5 \%$ FCS to a final concentration of 1000 cells $/ \mu \mathrm{l} .200 \mu \mathrm{l}$ of the cell suspension were transferred into ThinCert ${ }^{\odot}$ cell culture inserts with a pore size of $8 \mu \mathrm{m}$ (Greiner BioOne, Frickenhausen, Germany), which have been placed into a 24 -well plate provided with $600 \mu \mathrm{l}$ cell culture medium containing $10 \%$ FCS. After incubation for $24 \mathrm{~h}$ at $37^{\circ} \mathrm{C}$ and $5 \% \mathrm{CO}_{2}$, migrated cells at the outside bottom of the cell culture insert were detached by Trypsin/EDTA. Collected cells were centrifuged for 5 min at $2000 \mathrm{rpm}$, resuspended in $100 \mu$ I PBS and counted.

\subsubsection{Rac1 activation assay}

To determine the Rac1 activation of cells, the RhoA/Rac1/Cdc42 Activation Combo Biochem Kit ${ }^{\mathrm{TM}}$ (Cytoskeleton Inc., Denver, Colorado, USA) was used. For the analysis of mitotic cells, $2 \mu \mathrm{M}$ DME was added to the cell culture medium $16 \mathrm{~h}$ before cell lysis. Cell culture plates were washed once with $37^{\circ} \mathrm{C}$ warm PBS and were immediately placed on ice and lysed by scraping the cells after adding $1 \mathrm{ml}$ ice-cold lysis buffer (25 mM HEPES pH 7.5, $1 \%$ NP-40, $10 \mathrm{mM} \mathrm{MgCl}_{2}, 100 \mathrm{mM} \mathrm{NaCl}$, $5 \%$ glycerol, freshly supplemented with $1 \mathrm{mM} \mathrm{PMSF}, 1 \times$ protease inhibitor cocktail (Roche, Basel, Switzerland), as described in Briançon-Marjollet et al., 2008) per $10 \mathrm{~cm}$ dish. Subsequently, 
lysates were subjected to centrifugation at $14,800 \mathrm{rpm}$ for $1 \mathrm{~min}$ at $4^{\circ} \mathrm{C}$ to remove insoluble materials. Active Rac1 was pulled down by incubating the supernatant with $10 \mu \mathrm{g}$ GST-PAK-PBD beads for $30 \mathrm{~min}$ at $4^{\circ} \mathrm{C}$ and head-over-tail rotation. GST-PAK-PBD beads were washed two times with wash buffer (25 mM HEPES, pH 7.5, 1\% NP-40, $30 \mathrm{mM} \mathrm{MgCl,} 40 \mathrm{mM} \mathrm{NaCl}, 1 \mathrm{mM}$ dithiothreitol, as described in Briançon-Marjollet et al., 2008), resuspended in $15 \mu \mathrm{l}$ 5x SDS sample buffer and boiled for $5 \mathrm{~min}$ at $95^{\circ} \mathrm{C}$. Active Rac1 bound to PAK-PBD beads was detected on Western blots using mouse anti-Rac1 antibodies (1:800 in $3 \%$ BSA/TBS).

\subsubsection{GEF assay}

For determination of the Rac-GEF activity of cells, pull down assays with Rac1 G15A Agarose Beads (Cell Biolabs Inc., San Diego, California, USA) were performed. For the analysis of mitotic cells, $2 \mu \mathrm{M}$ DME was added to the cell culture medium $16 \mathrm{~h}$ before cell lysis. Cell culture plates were washed once with $37^{\circ} \mathrm{C}$ warm PBS and were immediately placed on ice and lysed by scraping the cells after adding $1 \mathrm{ml}$ ice-cold lysis buffer $(20 \mathrm{mM}$ TRIS-HCl pH 7.4, $100 \mathrm{mM} \mathrm{NaCl}$, $0.1 \% \mathrm{NP}-40,5 \mathrm{mM} \mathrm{MgCl}$, $1 \mathrm{mM}$ EDTA, freshly supplemented with $1 \mathrm{mM}$ DTT, $0.1 \%$ sodium desoxycholate, $20 \mathrm{mM} \mathrm{Na}_{3} \mathrm{VO}_{4}$, $25 \mathrm{mM}$ ß-Glycerophosphate, $50 \mathrm{mM} \mathrm{NaF}, 5 \mathrm{mM} \mathrm{Na} \mathrm{MoO}_{4}$ and 1x protease inhibitor cocktail) per $10 \mathrm{~cm}$ dish. Immediately, lysates were centrifuged at 14,800 rpm for $1 \mathrm{~min}$ at $4^{\circ} \mathrm{C}$ to remove debris. Active Rac-GEFs were pulled down by incubating lysate containing $5 \mathrm{mg}$ protein with $20 \mu \mathrm{g}$ Rac $1 \mathrm{G} 15 \mathrm{~A}$ Agarose Beads for $2 \mathrm{~h}$ at $4^{\circ} \mathrm{C}$ and head-over-tail rotation. Rac1 G15A Agarose Beads were washed twice with lysis buffer, resuspended in 5x SDS sample buffer and boiled for $5 \mathrm{~min}$ at $95^{\circ} \mathrm{C}$. Active TIAM1 or TRIO bound to Rac1 G15A Agarose Beads were detected on Western blots using mouse anti-TRIO (1:500 in $3 \%$ BSA/TBS) or rabbit anti-TIAM1 (1:1000 in $3 \%$ BSA/TBS) antibodies.

\subsubsection{GFP pull down assay}

To investigate the binding of TRIO-WT and TRIO-SRNN to EB1, GFP pull down assays were carried out. For this purpose, HEK293T cells were transfected with TRIO-WT-GFP, TRIO-SRNNGFP or GFP expressing plasmids via calcium phosphate transfection and HCT116 clones stably expressing TRIO-WT-GFP, TRIO-SRNN-GFP or GFP were generated. $16 \mathrm{~h}$ before lysis, $2 \mu \mathrm{M}$ DME were added to accumulate mitotic cells. For cell lysis, cells were placed on ice and $1 \mathrm{ml}$ or $1.5 \mathrm{ml}$ ice-cold lysis buffer (20 mM TRIS-HCl pH 7.4, $100 \mathrm{mM} \mathrm{NaCl}, 0.1 \%$ NP-40, 5 mM MgCl 2,1 mM EDTA, freshly supplemented with $1 \mathrm{mM}$ DTT, 0.1\% sodium desoxycholate, $20 \mathrm{mM} \mathrm{Na}_{3} \mathrm{VO}_{4}$, $25 \mathrm{mM} \beta$-Glycerophosphate, $50 \mathrm{mM} \mathrm{NaF}, 5 \mathrm{mM} \mathrm{Na}_{2} \mathrm{MoO}_{4}$ and $1 \times$ protease inhibitor cocktail) were added per $10 \mathrm{~cm}$ dish of HEK293T or $15 \mathrm{~cm}$ dish of HCT116 cells, respectively. $2 \mathrm{mg}$ lysate 
(HEK293T cells) or $10 \mathrm{mg}$ lysate (HCT116 cells) were mixed with $20 \mu \mathrm{l}$ or $40 \mu \mathrm{l}$ equilibrated GFP selector resin (NanoTag Biotechnologies, Göttingen, Germany) slurry and incubated for $1 \mathrm{~h}$ at $4^{\circ} \mathrm{C}$ with head-over-tail rotation. After washing three times with lysis buffer, GFP selector resin was resuspended in $5 \times$ SDS sample buffer and boiled for $5 \mathrm{~min}$ at $95^{\circ} \mathrm{C}$. TRIO-WT-GFP, TRIO-SRNNGFP and GFP as well as co-immunoprecipitated EB1 were detected on Western blots using mouse anti-GFP (1:3000 in $3 \%$ BSA/TBS) or mouse anti-EB1 (1:500 in $3 \%$ BSA/TBS) antibodies.

\subsubsection{Detection and quantification of actin cytoskeleton intensities}

\section{Live cell analysis}

For live cell analysis of actin cytoskeleton intensities, separated in the intensity of the F-actin cortex and the F-actin within the cell, hereafter referred to as actin clouds (Mitsushima et al., 2010), cells were transfected with $10 \mu \mathrm{g}$ pCS2-EGFP-hUtrophinCH and $10 \mu \mathrm{g}$ pcDNA-RFP-H2B via electroporation. Next day, cells were seeded into $\mu$-Slide 8 well chambers. $48 \mathrm{~h}$ after transfection, cells were washed once with PBS and phenol red-free cell culture medium was added. Live cell microscopy of metaphase cells was performed at $37^{\circ} \mathrm{C}$ and $5 \% \mathrm{CO}_{2}$ using a Delta Vision Elite ${ }^{\odot}$ microscope equipped with a PCO Edge sCMOS camera and an environmental chamber for temperature control and $\mathrm{CO}_{2}$ injection. Images were acquired with a z-optical spacing of $0.2 \mu \mathrm{m}$. Line scans of metaphase cells were set via softWoRx ${ }^{\circledR}$ Explorer 1.3.0 software. For calculation, background was defined as the mean pixel intensity of the 50 line scan pixels with the lowest intensity at one end of the line scan. Background was subtracted from overall mean pixel intensity. Actin cortex intensity was calculated as mean of the two cortex peak pixel intensities. Actin cloud intensity was calculated as mean of the pixel intensities in between the first low point after the first cortex peak and the last low point before the second cortex peak. To take different expression levels of EGFP-Utrophin into account, actin cortex intensity - initially assumed to stay unaffected by the given treatments - was used for normalization of actin cloud intensities. Per experiment at least 10 metaphase cells were evaluated.

\section{Analysis of fixed and stained cells}

For the analysis of actin cytoskeleton intensities of fixed cells, cells were seeded in $\mu$-Slide 8 wells and fixed with $4 \%$ PFA/PBS prewarmed to $37^{\circ} \mathrm{C}$ for $10 \mathrm{~min}$. After washing two times with PBS, cells were permeabilized with $0.1 \%$ Triton X-100/PBS for 5 min at RT, followed by two more washing steps with PBS and blocking with 1\% BSA/PBS for 30 min. For staining of F-actin, cells were incubated with $100 \mathrm{nM}$ Alexa Fluor ${ }^{\mathrm{TM}} 594$ Phalloidin (Thermo Fisher Scientific, Waltham, MA, USA) for $1.5 \mathrm{~h}$ in the dark at RT. Afterwards DNA was stained with Hoechst33342 (1:15,000 
in PBS) for 5 min at RT. After washing the cells three times with PBS, cells were left in PBS at $4^{\circ} \mathrm{C}$ until microscopy. Fixed and stained cells were analyzed using a Delta Vision Elite ${ }^{\odot}$ microscope equipped with a PCO Edge sCMOS camera and an Olympus 60x 1.40 NA objective. Images were acquired with a z-optical spacing of $0.2 \mu \mathrm{m}$. Line scans of metaphase cells were set via softWoRx ${ }^{\circledR}$ Explorer 1.3.0 software. For evaluation, background, actin cortex and cloud intensities were calculated as described in 'Live cell analysis'. Normalization of cortex as well as cloud intensities was done by setting each control group as 1 . Per experiment at least 20 metaphase cells were evaluated.

\subsubsection{Analysis of F-actin structures using STED microscopy}

In order to visualize extremely fine structures of the F-actin cytoskeleton, STED microscopy was carried out. For this purpose, cells grown on glass coverslips were fixed with $4 \%$ PFA/PBS prewarmed to $37^{\circ} \mathrm{C}$ for $10 \mathrm{~min}$. Afterwards, cells were washed two times with PBS, followed by permeabilization with $0.1 \%$ Triton X-100/PBS for 5 min at RT. Subsequently, cells were washed twice with PBS and blocked with 1\% BSA/PBS for $30 \mathrm{~min}$. For staining of microtubules, cells were incubated with mouse anti- $\alpha-T u b u l i n(1: 700)$ antibody diluted in 1\% BSA/PBS for 90 min at RT. Afterwards, cells were washed three times with PBS, followed by an incubation with anti-mouse Abberior $^{\circledR}$ STAR 580 secondary antibody (1:200, Abberior, Göttingen, Germany) and Abberior ${ }^{\circledR}$

STAR RED Phalloidin (1:200, Abberior, Göttingen, Germany) diluted in 1\% BSA/PBS for 90 min at RT. DNA was stained with Hoechst33342 (1:15,000 in PBS) for 5 min at RT. Finally, cells were washed three times with PBS and one time with Millipore water. Coverslips were air-dried and embedded using $10 \mu$ Abberior Mount Solid Antifade Medium (Abberior, Göttingen, Germany), preheated to $50^{\circ} \mathrm{C}$, per coverslip. While I was responsible for fixation and staining as well as the detection and identification of prometaphase and metaphase cells by fluorescence microscopy, Florian Grimm (Abberior GmbH, Göttingen, Germany) acquired images of those cells using an Abberior Instruments Expert Line STED microscope (Abberior Instruments, Göttingen, Germany), equipped with a continues wave (CW) excitation laser $(405 \mathrm{~nm})$, two pulsed excitation lasers (561 $\mathrm{nm}$ and $640 \mathrm{~nm})$ as well as an pulsed STED laser $(775 \mathrm{~nm})$, with an Olympus IX83 microscope body (Olympus, Tokyo, Japan) and an Olympus UPLSAPO 100xO/1.4 objective (Olympus, Tokyo, Japan). Top surface images of 15 z-stacks (pixel size $20 \mathrm{~nm}$, dwell time $10 \mu \mathrm{s}$ ) with a z-optical spacing of $0.2 \mu \mathrm{m}$ were acquired and maximum projection was chosen to convert the $z$-stacks into 2D images using the Imspector 16.1 software (Abberior Instruments, Göttingen, Germany). 


\subsubsection{AFM mechanical measurements}

For the analysis of the cortical tension of prometaphase cells, AFM indentation experiments were performed. To enable the identification of prometaphase cells, cells were transfected with $10 \mu \mathrm{g}$ pEGFP-H2B by electroporation. Next day, $2.5 \times 10^{5}$ cells were seeded into $\mu$-Dishes ( $35 \mathrm{~mm}$, low Grid-500, ibidi, Martinsried, Germany) and $48 \mathrm{~h}$ after transfection medium was supplemented with 15 mM HEPES. Live cell analysis was carried out by Dr. Bastian Rouven Brückner (Institute for Biophysical Chemistry, Group of Prof. Janshoff, Georg August University Göttingen), Nadine Schermuly (Institute for Molecular Onkology, Group of Prof. Bastians, University Medical Center Göttingen) and me, whereby in alternate order Nadine Schermuly and I were responsible for the detection and identification of prometaphase cells by fluorescence microscopy and Dr. Bastian Rouven Brückner carried out the preparation of pyramidal AFM probes (MLCT, Bruker AFM Probes, Camarillo, USA), AFM measurements and the analysis of force-indentation curves for the calculation of cortical tension $T_{0}$ and membrane tension $T_{t}$. For probe preparation, probes were rinsed three times with isopropanol and PBS--, followed by an incubation with concanavalin AFITC conjugate $(2.5 \mathrm{mg} / \mathrm{ml}$ in PBS--, Sigma-Aldrich, Steinheim, Germany) for $1.5 \mathrm{~h}$ at RT. This procedure allowed for a strong binding between the cell's membrane and the probe required for pulling out plasma membrane tethers and, thus, for the determination of membrane tension. By using the thermal noise method (Hutter \& Bechhoefer, 1993), the exact spring constant of each cantilever was determined. For recording force-indentation cycles, the cantilever tip was placed at the center of a prometaphase cell. Every force-indentation cycle started with an indentation up to a maximum force of $F_{\max }=1 \mathrm{nN}$, at which position the cantilever was kept for a dwell time of $0.5 \mathrm{~s}$, followed by retraction of the probe using a pulling velocity of $2 \mu \mathrm{m} / \mathrm{s}$. Per experiment, about 10 force-indentation cycles per cell and a total of 20 cells per treatment in at least three independent experiments were measured.

Force-indentation curves were analyzed by Dr. Bastian Rouven Brückner by fitting indentation data that starts at the contact point between the cell and the probe using following formula with $F$ is the force and $\delta$ is the indentation depth:

$$
F(\delta)=a \cdot \delta+b \cdot \delta^{3}
$$

To calculate the cortical tension $T_{0}$ of a cell, prefactor a was used as well as $\Theta=\frac{\pi}{2}-\phi$ to take the geometrical properties of the indenter with the half-opening angle $\phi$ into account:

$$
T_{0}=\frac{a}{2 \pi \cos (\Theta)}
$$




\subsubsection{Actin cortex thickness measurements}

For the determination of actin cortex thickness, cells were prepared and images were acquired as described for fixed cells in 2.2.12 "Detection and quantification of actin cytoskeleton intensities". The software for actin cortex thickness analysis was written and kindly provided by Prof. Dr. Burkhard Geil (Institute for Biophysical Chemistry, Georg August University Göttingen). Tiff-files of images only showing the phalloidin staining of single metaphase cells were loaded into the software. In order to avoid a possible impact of the F-actin signal intensity, this was normalized by setting the minimal pixel intensity of every image to 0 and the maximal pixel intensity of every image to 1 . The image was adapted by using a Gaussian function with $\sigma=2$. To take the high variation in the background intensity into account, adaptive thresholding was used with a block size of 21 and an offset of 0.025 . In order to take the square size of a pixel into account for the calculation of the diameter at every point of the cortex the Euclidian distance was determined, followed by skeletonization. Finally, the pixel size was converted into $\mu \mathrm{m}$ ( 1 pixel $=0.108 \mu \mathrm{m}$ ) and the mean diameter of all calculated cortex skeleton points was determined.

\subsection{Statistics}

For statistical analysis of data from Rac1 activation assays, phospho-PAK1/2 levels, GEF assays and GFP pull down assays, differences in protein loading were corrected using the data that represented the expression level of housekeeping genes ( $\alpha$-tubulin, $\beta$-actin) or the input level (EB1, Rac1, TRIO). Normalization of biological replicates was performed by sum of all data points in a replicate (Degasperi et al., 2014). An unpaired $t$-test was applied to all graphs with $p$-values indicated as: ns (not significant): $p>0.05,{ }^{*}: p \leq 0.05,{ }^{* *}: p \leq 0.01,{ }^{* * *}: p \leq 0.001,{ }^{* * *}: p \leq 0.0001$.

\subsection{Protein Biochemistry}

\subsubsection{Preparation of protein lysates}

For lysing proteins, cells were washed one time with PBS, followed by detachment using $0.5 \mathrm{mM}$ PBS/EDTA. Alternately, cells were placed on ice and detachment was performed by using a cell scraper. Detached cells were centrifuged for $5 \mathrm{~min}$ at $2000 \mathrm{rpm}$ and cell pellet was resuspended in 50-100 $\mu$ l lysis buffer (50 mM TRIS-HCl pH 7.4, 150 mM NaCl, 5 mM EDTA, 5 mM EGTA, $1 \%$ $(\mathrm{v} / \mathrm{v}) \operatorname{Igepal}^{\odot}, 0.1 \%(\mathrm{w} / \mathrm{v})$ SDS, freshly supplemented with $0.1 \%$ sodium desoxycholate, $20 \mathrm{mM}$ 
$\mathrm{Na}_{3} \mathrm{VO}_{4}, 25 \mathrm{mM}$-Glycerophosphate, $50 \mathrm{mM} \mathrm{NaF}, 5 \mathrm{mM} \mathrm{Na}_{2} \mathrm{MoO}_{4}, 1 x$ protease inhibitor cocktail and $0.5 \mu \mathrm{M}$ microcystin-LR (Enzo Life Science, Lörrach, Germany)) per amount of cells grown in a 6-well. After $15 \mathrm{~min}$ incubation on ice, cell lysates were subjected to centrifugation for $15 \mathrm{~min}$ at $14,800 \mathrm{rpm}$ at $4^{\circ} \mathrm{C}$. The supernatant was transferred into a new reaction tube and used for protein determination and/or stored at $-20^{\circ} \mathrm{C}$ until further use. For checking the transfection efficiency, lysate containing $50 \mathrm{\mu g}$ of protein was supplemented with $5 x$ SDS sample buffer $(50 \%$ glycerol, $15 \%$ (v/v) $\beta$-mercaptoethanol, $15 \%$ (w/v) SDS, $0.25 \%$ (w/v) bromophenol blue). Denaturation of proteins was achieved by boiling the samples for $5 \mathrm{~min}$ at $95^{\circ} \mathrm{C}$. Samples were stored at $-20^{\circ} \mathrm{C}$ until further use.

\subsubsection{Protein determination}

The protein concentration of cell lysates was determined by using the Bio-Rad DC ${ }^{\mathrm{TM}}$ Protein Assay (BioRad, Hercules, California, USA) according to the manufacturer's protocol. Photometric measurements were carried out at a $\operatorname{VICTOR}^{\odot} \mathrm{X} 3$ microplate reader.

For all experiments, protein determination of cell lysates was performed and equal amounts of proteins were used for detection via Western blots, including IPs, Rac1 and GEF assays.

\subsubsection{Sodium dodecyl sulfate polyacrylamide gel electrophoresis (SDS-PAGE)}

In order to separate denatured proteins from cell lysates by their molecular weights, a discontinuous SDS-PAGE was used. The SDS gel was composed of $5 \%$ stacking gel (300 nM TRIS-HCl, $\mathrm{pH}$ 6.8, $0.1 \%$ (w/v) SDS, $5 \%$ (v/v) Rotiphorese Gel 30 ${ }^{\odot}$ (Carl Roth, Karlsruhe, Germany)) and 7-13 \% resolving gel (500 nM TRIS-HCl, pH 8.8, $0.1 \%$ (w/v) SDS, 7-13 \% (v/v) Rotiphorese $\mathrm{Gel} 30^{\odot}$ ). The percentage of the resolving gel was dependent on the molecular weight of the proteins to be detected. Gradient gels consisting of a two-part resolving gel with different percentages were used for the detection of proteins with strongly diverging molecular weights. Prepared protein lysates and $5 \mu \mathrm{l}$ of PageRuler Prestained Protein Ladder (Fermentas, St. LeonRot, Germany) for proteins up to $180 \mathrm{kDa}$, PageRuler Plus Prestained Protein Ladder (Fermentas, St. Leon-Rot, Germany) for proteins up to $245 \mathrm{kDa}$ or ProSieve ${ }^{\mathrm{TM}}$ QuadColor ${ }^{\mathrm{TM}}$ Protein Marker (Lonza Group Ltd., Basel, Switzerland) for proteins up to $300 \mathrm{kDa}$ or were loaded onto the SDS gel. Protein separation was carried out in SDS running buffer (25 mM TRIS-HCl, pH 6.8, $192 \mathrm{mM}$ glycine, $0.15 \%(w / v)$ SDS) for $1 \mathrm{~h}$ at $27 \mathrm{~mA}$ and $2-3 \mathrm{~h}$ at $37 \mathrm{~mA}$. 


\subsubsection{Western blot}

\section{Semi-dry blot}

In order to transfer proteins smaller than $100 \mathrm{kDa}$, a semi-dry blot was mostly used. For protein transfer, the SDS gel was placed onto a nitrocellulose membrane and wetted with transfer buffer (24.8 mM TRIS-HCl pH 8.0, 170 mM (v/v) glycine, $0.0025 \%$ (w/v) SDS, $20 \%$ methanol). Blotting was performed at $200 \mathrm{~mA}$ for $1.5 \mathrm{~h}$.

\section{Tank blot}

For electrophoretical transfer especially of proteins larger than $100 \mathrm{kDa}$, a tank blot system was used. Protein transfer from the SDS gel to a nitrocellulose or PVDF (protein transfer and immobilization on a polyvinylidene fluoride) membrane, with the latter one being activated by an incubation in methanol followed by intensive washing with water, took place in a tank-blot chamber. Blotting was performed in transfer buffer $(24.8 \mathrm{mM}$ TRIS- $\mathrm{HCl}$ pH 8.0, $170 \mathrm{mM}(\mathrm{v} / \mathrm{v})$ glycine, $0.0025 \%(w / v)$ SDS, $15 \%$ methanol) at $450 \mathrm{~mA}$ for $3 \mathrm{~h}$.

After protein transfer, the membrane was blocked with $5 \%$ milk powder/TBS $(50 \mathrm{mM}$ TRIS-HCl, $\mathrm{pH} 7.2,160 \mathrm{mM} \mathrm{NaCl}$ ) for $30 \mathrm{~min}$ at RT. Proteins of interest were labelled by overnight incubation with specific primary antibodies diluted in $3 \% \mathrm{BSA} / \mathrm{TBS}$ at $4^{\circ} \mathrm{C}$ shaker. Then the membrane was rinsed 2 times with $0.1 \%$ TBS-T $(0.1 \%$ Tween in TBS) and then washed 3 time for 10 min each with $0.1 \%$ TBS-T on a shaker. Afterwards, the membrane was incubated with the appropriate horseradish peroxidise (HRP) conjugated secondary antibody diluted in $3 \%$ milk powder/TBS for $1.5 \mathrm{~h}$, followed by washing as described above. For protein detection, the membrane was treated with freshly prepared enhanced chemiluminescence (ECL) solution $(0.1 \mathrm{mM}$ TRIS- $\mathrm{HCl}, \mathrm{pH} 8.5$, $2.5 \mathrm{mM}$ luminol, $0.4 \mathrm{mM} \beta$-coumaric acid, $\left.0.03 \%(\mathrm{v} / \mathrm{v}) \mathrm{H}_{2} \mathrm{O}_{2}\right)$ or with Amersham ECL Western Blotting Detection Reagent (GE Healthcare, Chalfont St. Giles, UK). Finally, chemiluminescence was measured using a Fusion-SL 3500-WL chemiluminescence imaging system (Peqlab, Erlangen, Germany). A digital image was taken of the protein marker. 


\subsection{Molecular Biological Methods}

\subsubsection{Escherichia coli (E. coli) cells}

For transformation of plasmid DNA, the following E. coli stain was used:

$\mathrm{DH} \mathrm{aF}^{-} \phi 80$ lacZ $\triangle \mathrm{M} 15 \triangle($ lacZYA-argF $) \mathrm{U} 169$ deoR recA1 $h s d R 17\left(\mathrm{r}_{\mathrm{k}}^{-}, \mathrm{m}_{\mathrm{k}}^{+}\right)$phoA supE44 thi-1 gyrA96 relA1ג'.

\subsubsection{Preparation of competent E. coli}

To prepare chemically competent $E$. coli cells, $5 \mathrm{ml}$ Luria Bertani (LB) medium were inoculated with E. coli $\mathrm{DH} 5 \mathrm{a}$ and incubated overnight at $37^{\circ} \mathrm{C}$ shaking at $110 \mathrm{rpm}$. The next morning the overnight culture was added to $400 \mathrm{ml}$ LB medium and incubated shaking at $37^{\circ} \mathrm{C}$ until $\mathrm{OD}_{600}$ was approximately 0.5 . After $5 \mathrm{~min}$ incubation on ice, cells were centrifuged for $5 \mathrm{~min}$ at $2000 \mathrm{rpm}$. The cell pellet was resuspended in $40 \mathrm{ml}$ Tfbl buffer (30 mM potassium acetate, $100 \mathrm{mM} \mathrm{RbCl}, 10 \mathrm{mM}$ $\mathrm{CaCl}_{2}, 15 \%(\mathrm{v} / \mathrm{v})$ glycerol, $\mathrm{pH}$ 6.0) and incubated on ice for $5 \mathrm{~min}$, followed by centrifugation at $2000 \mathrm{rpm}$ for $5 \mathrm{~min}$. This time, cells were resuspended in $4 \mathrm{ml}$ Tfbll buffer (10 mM MOPS, $75 \mathrm{mM}$ $\mathrm{CaCl}_{2}, 10 \mathrm{mM} \mathrm{RbCl}, 15 \%$ glycerol, $\mathrm{pH}$ 6.5) and incubated on ice for $15 \mathrm{~min}$. Aliquots of $100 \mu \mathrm{l}$ were snap frozen in liquid nitrogen and stored at $-80^{\circ} \mathrm{C}$.

\subsubsection{Transformation of $E$. coli cells}

To transfer plasmids into competent of E. coli DH5a cells, 1-2 $\mu$ g of plasmid DNA was added to $50 \mu \mathrm{l}$ competent cells and gently mixed by pipetting up and down. After 20 min incubation on ice, cells were subjected to a heat shock at $42^{\circ} \mathrm{C}$ for $1 \mathrm{~min}$. Subsequently, $600 \mu \mathrm{LB}$ medium was added and cells were incubated for $45-60 \mathrm{~min}$ at $37^{\circ} \mathrm{C}$ and $650 \mathrm{rpm}$ shaking. Afterwards, the cell suspension was transferred to $400 \mathrm{ml}$ LB medium supplemented with the appropriate selective antibiotic (100 mg/ml ampicillin or $50 \mathrm{mg} / \mathrm{l}$ kanamycin) and incubated overnight at $37^{\circ} \mathrm{C}$ in an incubation shaker at $110 \mathrm{rpm}$. Cells were harvested by centrifugation for $20 \mathrm{~min}$ at $4000 \mathrm{rpm}$ and cell pellets were stored at $-20^{\circ} \mathrm{C}$ until further processing.

\subsubsection{Plasmid Isolation}

Plasmid DNA purification was carried out using the NucleoBond ${ }^{\odot}$ PC 100 X-TRA Midi Kit (Macherey-Nagel GmbH \& Co. KG, Düren, Germany) according to the manufacturer's protocol. The plasmid DNA concentration was determined by using the NanoDrop ${ }^{\odot} 2000$ spectrophotometer. 


\section{Results}

\subsection{TRIO binds to EB1 at microtubule plus ends in a microtubule dynamic dependent manner}

Recent studies as well as work from our group gave rise to a putative pathway, that connects microtubule dynamics to the mitotic actin cortex and that seems to be involved in the generation of spindle mis-positioning, chromosome mis-segregation and thus chromosomal instability when being hyperactivated. This pathway includes microtubule plus-end bound EB1, the GEF TRIO and its Rho GTPase Rac1, as well as the actin cytoskeleton remodeling Arp2/3 complex. So far it has been shown that microtubule plus-end assembly rates and EB1 are associated with TRIO activity (Berger, 2016), but not whether this is due to a direct interaction or whether further interaction partners are involved. In order to figure this out, EB1-TRIO interaction studies were performed, including looking for co-localization via immunofluorescence and performing pull down assays. Since van Haren et al. (2014) only showed co-localization of EB1 and overexpressed TRIO together with overexpressed Navigator 1 (NAV1), a microtubule plus-tip binding TRIO-interaction partner, evidence for co-localization of endogenously expressed EB1 and TRIO at microtubule plus-tips was still lacking. Unpublished immunofluorescence data from our group shows that TRIO is indeed microtubule-associated in chromosomally unstable SW620 in mitosis, and that this localization is EB1 dependent as it is abolished after EB1 knock down. Further immunofluorescence staining and microscopy using anti-EB1 and anti-TRIO antibodies in chromosomally stable HCT116 and chromosomally unstable SW620 colorectal cancer cells proved the co-localization of EB1 and TRIO and it was observed in interphase cells as well as in different mitotic phases (Figure 3.1a). Although the images are not sufficient to visualize a clear microtubule plus-tip localization of TRIO, the co-localization with EB1 as well-known microtubuleend binding protein strongly suggests TRIOs microtubule plus-tip localization. In both cell lines in interphase and mitosis, the microtubule-associated localization of TRIO was strongly reduced after siRNA-mediated depletion of EB1, thereby demonstrating the EB1 dependence and further confirming the unpublished data from our group (Figure 3.1b). However, there was not only less microtubule-associated TRIO, but also an overall reduction of TRIO observed in EB1-depleted cells. This might be explained by a reduced stability of unbound TRIO when EB1 is not present or by washout of unbound TRIO during the methanol-fixation step of the staining procedure.

Due to uncovering the role of microtubule plus-end assembly rates for CIN development, chromosomally stable HCT116 cells were treated with low dose of the microtubule-affecting drug Nocodazole. Nocodazole has been shown to increase microtubule plus-end assembly rates when 
used in subnanomolar concentrations (Ertych et al., 2014). In contrast, chromosomally unstable SW620 cells were treated with low dose of the microtubule-affecting drug Taxol, which in turn has a microtubule plus-end assembly increasing effect when used in subnanomolar concentrations. However, neither Nocodazole treatment of HCT116 cells showed an increasing, nor Taxol treatment of SW620 cells showed a reducing effect on the amount of EB1 or TRIO located at microtubule plus-tips (Figure 3.1b). This result indicates that there is no effect of microtubule plusend assembly rates on the microtubule plus-tip localization of EB1 and TRIO.

a

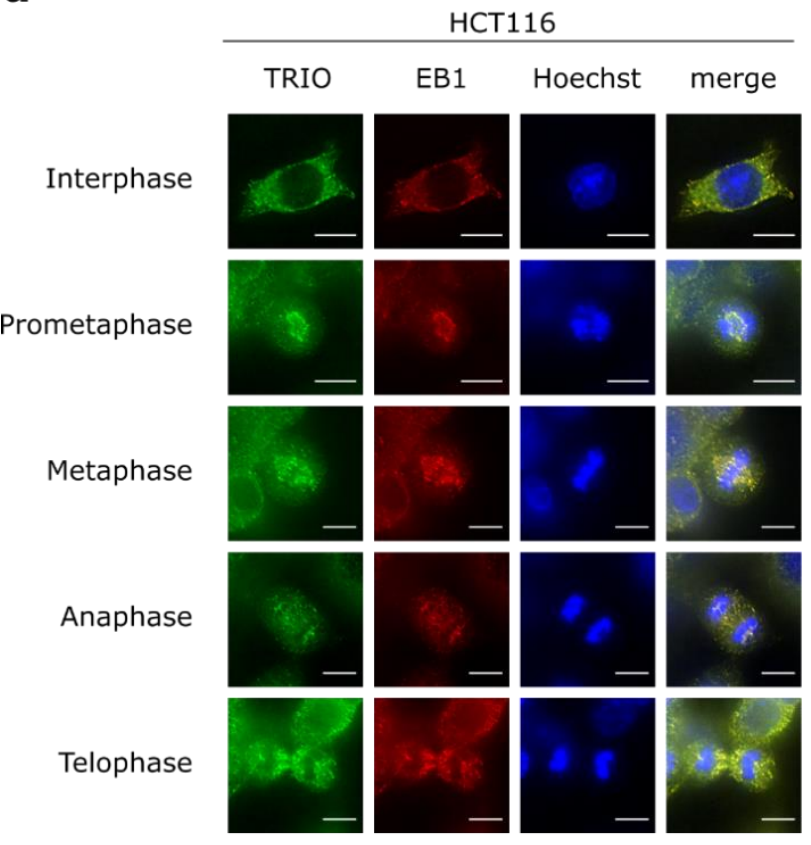

b

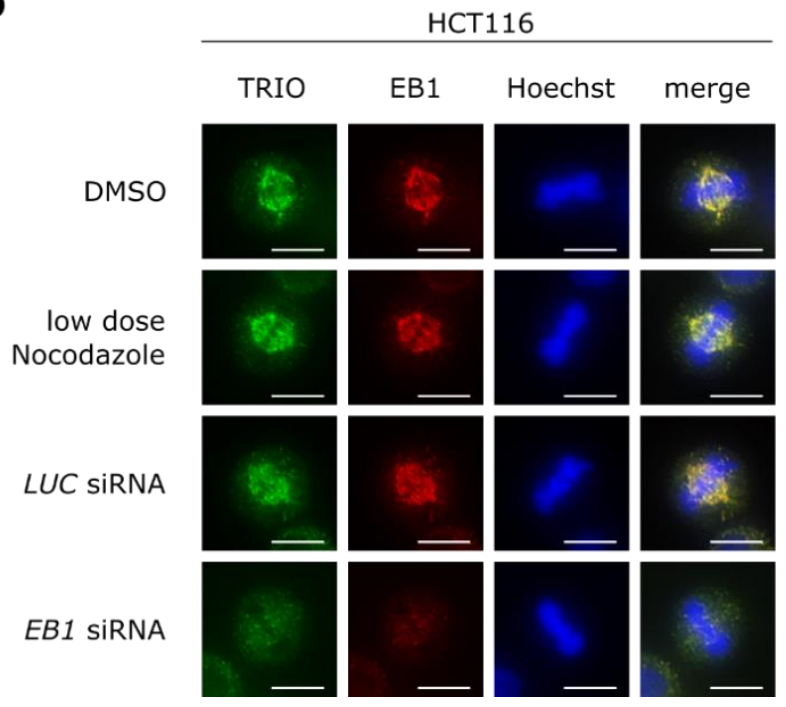

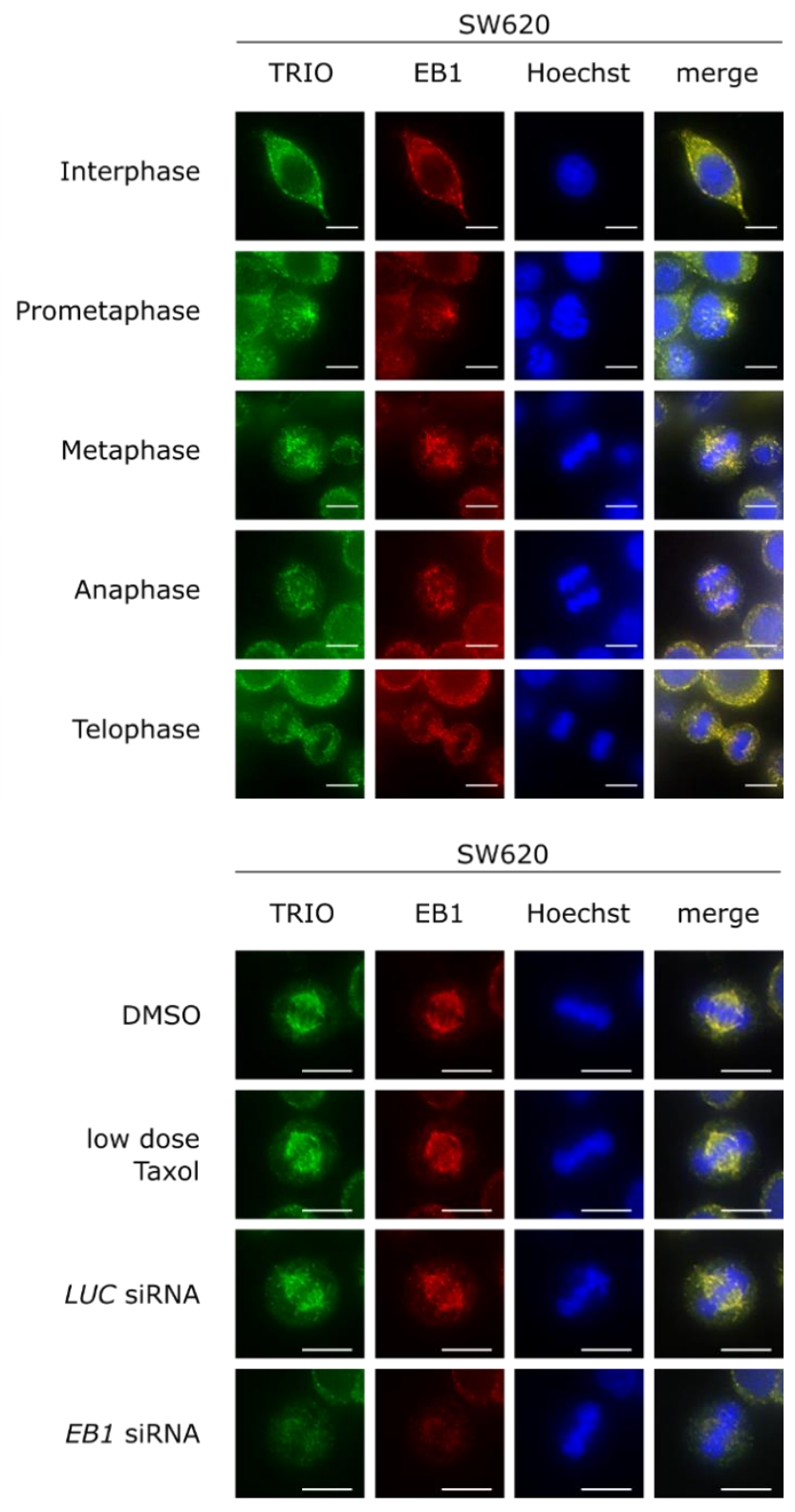


Figure 3.1: TRIO co-localizes with EB1 at microtubules in an EB1 dependent manner. Representative immunofluorescence images of chromosomally stable HCT116 and chromosomally unstable SW620 cells showing localization of endogenous EB1 and TRIO in (a) interphase and several mitotic phases and (b) after treatment with microtubule plus-end assembly rate-affecting drugs $(0.2 \mathrm{nM}$ Taxol or $0.5 \mathrm{nM}$ Nocodazole for $16 \mathrm{~h}$ ) or siRNA against Luciferase (LUC) or EB1 in metaphase cells. Cells were stained for TRIO (green), EB1 (red) and Hoechst33342 (DNA; blue). Scale bar $10 \mu \mathrm{m}$.

However, a co-localization does not automatically mean that there is also an interaction between EB1 and TRIO, which might be still be affected by microtubule dynamics. In order to confirm the EB1-TRIO interaction, postulated by van Haren et al. (2014), and to look for a possible impact of microtubule dynamics on this interaction, pull down assays were performed. Since EB1-TRIO coimmunoprecipitation assays with endogenously expressed EB1 and TRIO did not prove EB1TRIO binding, an overexpression system was chosen to ensure that enough binding partners were available to be detected in pull down experiments. Due to the much higher endogenous expression of EB1 compared to TRIO, TRIO-GFP was used for overexpression and pull down via GFP selector beads. The large size of TRIO-GFP - the sequence of TRIO-GFP consists of about 10 $\mathrm{kb}$ - required an overexpression system that was easy transfectable with large plasmids. For this reason, HEK293T cells were chosen and transfected with plasmids expressing TRIO-WT-GFP or TRIO-SRNN-GFP, with the latter one having a mutated SXIP sequence motif which is critical for EB1-binding (van Haren et al., 2014). $16 \mathrm{~h}$ before pull downs, cells were treated with low dose Taxol or Nocodazole as well as with the Eg5 inhibitor dimethylenastrone (DME) for the induction of mitotic arrest. Pull downs of TRIO-WT-GFP and TRIO-SRNN-GFP via GFP sector beads were analyzed by Western blots together with bound endogenous EB1 (Figure 3.2a). Levels of bound EB1 were quantified and the results not only confirmed the requirement of the SXIP motif of TRIO for EB1 binding, but also showed that low dose Taxol treatment led to a reduction of EB1 bound to TRIO-WT-GFP (Figure 3.2b). This strongly suggests an impact of microtubule plus-end assembly rates on the EB1-TRIO interaction. However, only low dose Taxol treatment, but not low dose Nocodazole treatment caused a significant change in bound EB1 levels. Ertych et al. (2014) has shown, that CIN cells have increased microtubule plus-end assembly rates that can be decreased by low dose Taxol and that non-CIN cells exhibit normal microtubule plus-end assembly rates that can be increased by low dose Nocodazole. Consequently, GFP pull down results suggested HEK293T cells to be chromosomally unstable. Chromosomal instability of HEK293T cells was already reported by Stepanenko and Dmitrenko (2015), but the microtubule plus-end assembly rate of mitotic HEK293T cells was still unknown. For this purpose, HEK293T cells were transfected with a plasmid for expression of the GFP-tagged microtubule plus-endbinding protein EB3, which could be tracked in a temporal sequence of fluorescent live cell images 
a

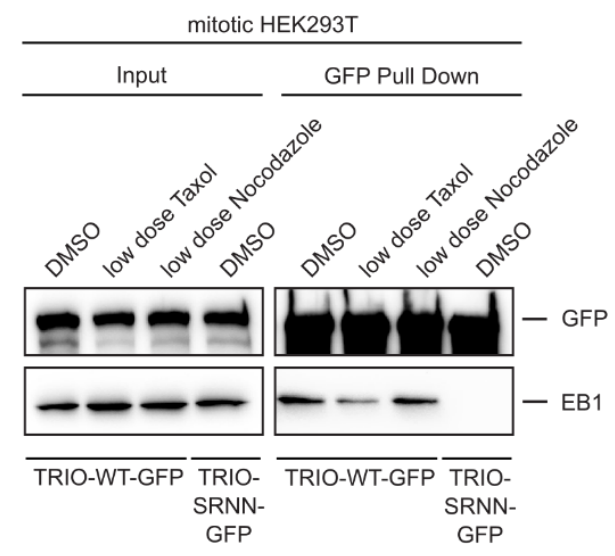

b

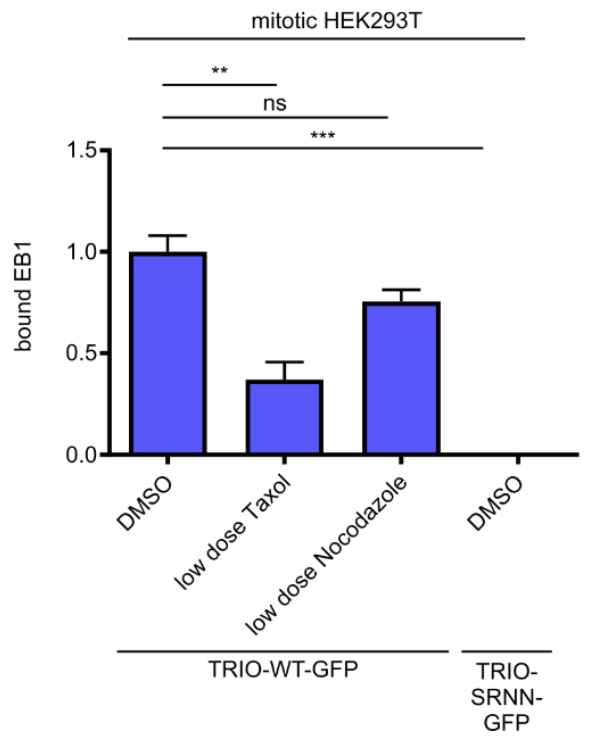

C

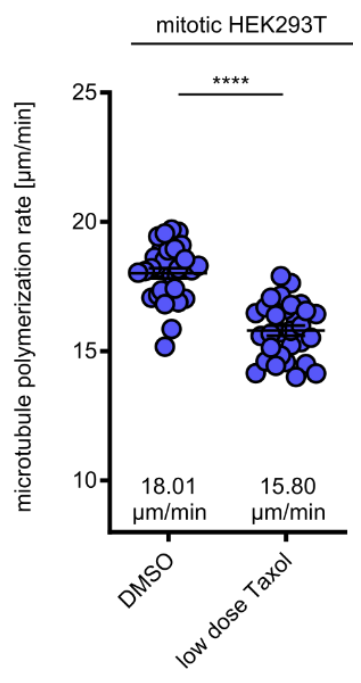

Figure 3.2: Interaction between TRIO and EB1 can be diminished by a decrease in microtubule plusend assembly rates in chromosomally unstable HEK293T cells. (a) Representative Western blot of GFP pull down from mitotic HEK293T cells overexpressing TRIO-WT-GFP or EB1 binding deficient TRIO-SRNNGFP showing a reduced amount of EB1 bound to TRIO-WT-GFP in cells with decreased microtubule plusend assembly rates induced by low dose Taxol treatment. Treatment with low dose Nocodazole did not show a significant effect. HEK293T cells were transfected with TRIO-WT-GFP or TRIO-SRNN-GFP expressing plasmids and treated with DMSO (control), $0.2 \mathrm{nM}$ Taxol or $0.5 \mathrm{nM}$ Nocodazole together with $2 \mu \mathrm{M}$ DME for mitotic arrest for $16 \mathrm{~h}$. GFP selector beads were used for pull down of TRIO-WT-GFP or TRIO-SRNN-GFP and bound endogenous EB1 was detected on Western blots. For detection, anti-GFP and anti-EB1 antibodies were used. (b) Quantification of EB1 levels from three independent GFP pull down experiments (mean \pm SEM, $t$-test). (c) HEK293T cells show slightly increased microtubule plus-end assembly rates that are reduced to normal by treatment with low dose Taxol. $48 \mathrm{~h}$ before measurement of microtubule plus-end assembly rates, HEK293T cells were transfected with an EB3-GFP expressing plasmid. $2 \mathrm{~h}$ prior to measurement, $0.2 \mathrm{nM}$ Taxol and $2 \mu \mathrm{M}$ DME were added. Scatter dot plots show mean values \pm SEM of 20 microtubules analyzed per cell with a total of 10 cells per experiment $(n=3, t$-test).

used for the calculation of microtubule plus-end assembly rates. As presumed, mitotic HEK293T cells showed an increased microtubule plus-end assembly rate of $18 \mu \mathrm{m} / \mathrm{min}$, that could be decreased to $15.8 \mu \mathrm{m} / \mathrm{min}$ meaning to a non-CIN level by low dose Taxol treatment (Figure 3.2c). This rescue by low dose Taxol treatment goes along with the result from the GFP pull down experiments. Together, these results show that there is a SXIP motif dependent and microtubule dynamic dependent EB1-TRIO interaction in mitotic chromosomally unstable cells, that is diminished after rescue of microtubule plus-end assembly rates. From this it can be suggested that a rescue of chromosomal instability due to a rescue of microtubule plus-end assembly rates in chromosomally unstable cells might be the consequence of a reduced EB1-TRIO interaction downstream of microtubule dynamics.

Vice versa, the abovementioned results would suggest a lower EB1-TRIO interaction in chromosomally stable cells that show normal microtubule plus-end assembly rates. In order to test 
this assumption, HCT116 cell clones stably expressing EGFP, TRIO-WT-GFP or TRIO-SRNNGFP were generated, which due to the vast size of full-length TRIO has proven to be particularly difficult in the past not only in our group (Seipel et al., 1999). Expression of TRIO-WT-GFP and TRIO-SRNN-GFP of three independent cell clones was verified by Western blots (Figure 3.3a). Live cell images of generated HCT116 cell clones transiently co-expressing EB1-mCherry were difficult to interpret in terms of EB1-TRIO co-localization due to the high cytoplasmic background signal of transfected cells (Figure 3.3b). However, in contrast to GFP, both TRIO-WT-GFP and TRIO-SRNN-GFP showed clear localization at lamellipodia-like structures of interphase cells, which underlines the function of TRIO in lamellipodia formation that has already been described by Seipel et al. (1999). According to findings from van Rijssel et al. (2012), this function of TRIO in lamellipodia formation is also associated with cell migration. Localization of TRIO-SRNN-GFP at lamellipodia-like structures showed to be even more pronounced than that of TRIO-WT-GFP. Both, TRIO-WT-GFP and TRIO-SRNN-GFP expressing cells also seemed to have larger lamellipodia than GFP expressing cells, suggesting a possible effect on the migrative behavior. To confirm the observed structures as lamellipodia, an additional staining of lamellipodial markers and/or the F-actin cytoskeleton would be useful. Before being further analyzed, microtubule plusend assembly rates of the generated HCT116 clones were determined to exclude an effect of TRIO itself on microtubule dynamics. Both, TRIO-WT-GFP and TRIO-SRNN-GFP overexpression did not have any effect on microtubule plus-end assembly rates, thereby verifying TRIO to be downstream of microtubule dynamics (Figure 3.3c).

Now knowing that HCT116 clones stably expressing GFP, TRIO-WT-GFP or TRIO-SRNN-GFP exhibit normal microtubule plus-end assembly rates, these cells were used for GFP pull down experiments. In order to test, whether an increase of microtubule plus-end assembly rates would alter the level of EB1-TRIO interaction in mitotic HCT116 clones, these cells were treated with low dose Nocodazole and DME $16 \mathrm{~h}$ before GFP pull down. Quantification of bound endogenous EB1 on Western blots again confirmed the requirement of the SXIP motif of TRIO for EB1 binding, but did not show an effect of low dose Nocodazole treatment on the amount of EB1 bound to TRIOWT-GFP (Figure 3.4). Whether the small band representing bound EB1 in TRIO-SRNN-GFP expressing HCT116 cells is due to unspecific binding or experimental reasons needs to be further tested by additionally performing pull downs of GFP expressing HCT116 control clones.

Summed up, the overall GFP pull down results demonstrate that there is a SXIP motif dependent EB1-TRIO interaction that can be reduced by Taxol-induced decrease but not enhanced by Nocodazole-induced increase of upstream microtubule plus-end assembly rates. Further research would be necessary to reveal a more detailed picture of the role of microtubule dynamics on EB1- 
TRIO interaction. This includes, inter alia, testing whether the microtubule-affecting impact of Nocodazole might be somehow restricted by TRIO overexpression in HCT116 clones.

a

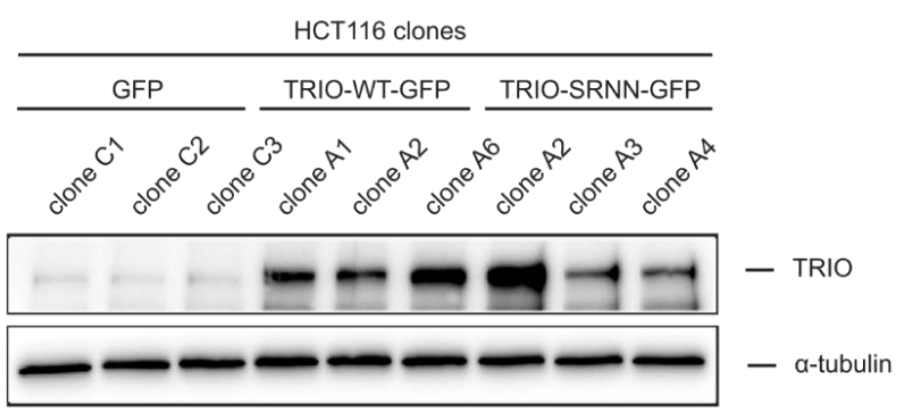

b

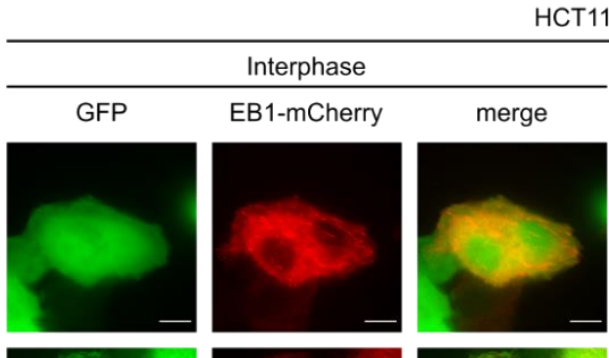

HCT116 clones

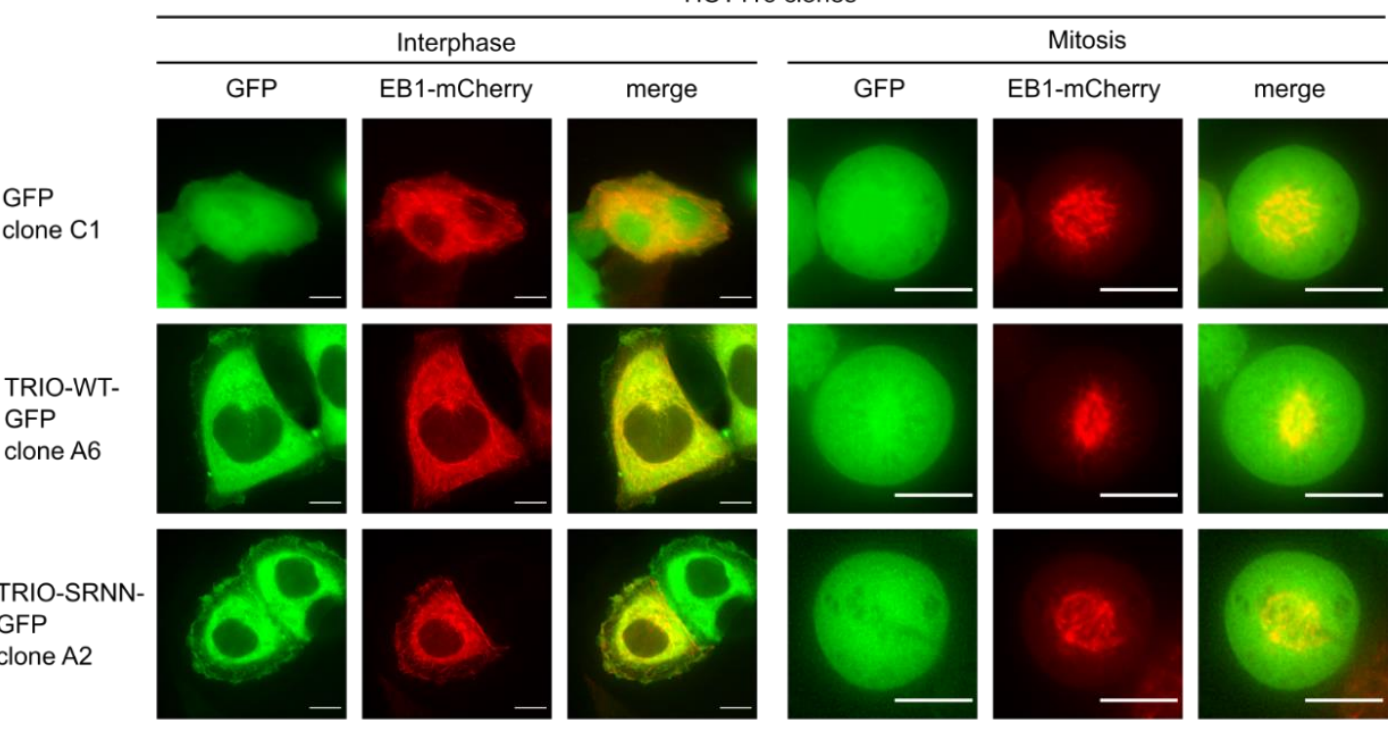

C

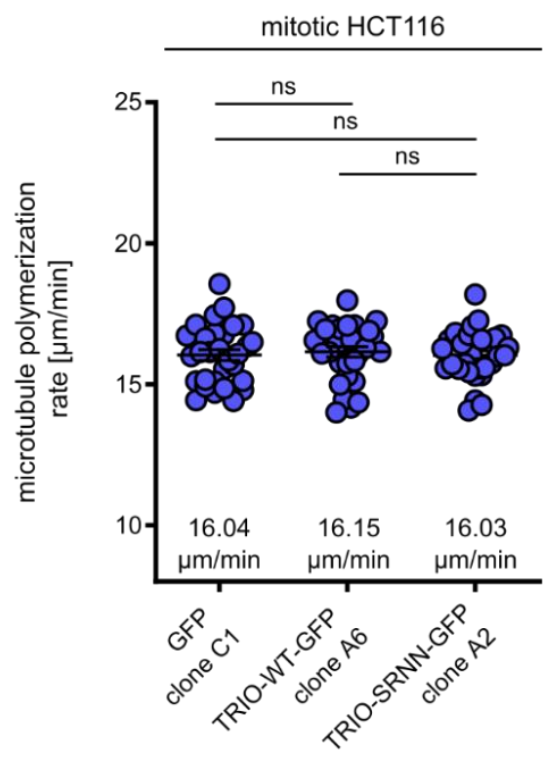


Figure 3.3: Generation of HCT116 cell clones stably expressing GFP, TRIO-WT-GFP or TRIO-SRNNGFP. (a) Representative Western blot of three independent HCT116 cell clones stably expressing GFP, TRIO-WT-GFP or TRIO-SRNN-GFP respectively. HCT116 cells have been transfected with corresponding plasmids using Lipofectamine ${ }^{\mathrm{TM}} 3000$ and stably transfected cells have been selected using G418. GFPpositive single cell clones were picked and used for further analysis. Expression levels were detected on Western blots via an anti-TRIO antibody. Anti- $\alpha$-tubulin antibody was used to test for equal protein loading. (b) Representative live cell images of interphase and mitotic HCT116 cell clones stably expressing GFP, TRIO-WT-GFP or TRIO-SRNN-GFP together with transiently expressed EB1-mCherry. HCT116 clones have been subjected to electroporation with pmCherry-EB1 plasmid $48 \mathrm{~h}$ before microscopy. Scale bar $10 \mu \mathrm{m}$. (c) Stable expression of TRIO-WT-GFP or TRIO-SRNN-GFP does not affect microtubule plus-end assembly rates. HCT116 clones were transfected with pmCherry-EB3 plasmid $48 \mathrm{~h}$ before and $2 \mu \mathrm{M}$ DME were added $2 \mathrm{~h}$ before measurements. Scatter dot plots show average microtubule growth rates from 30 cells from three independent experiments (mean \pm SEM, $t$-test).

a

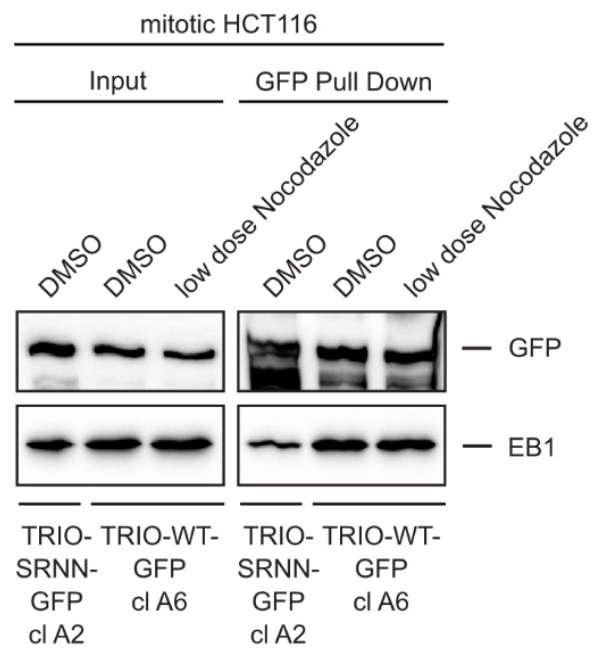

b

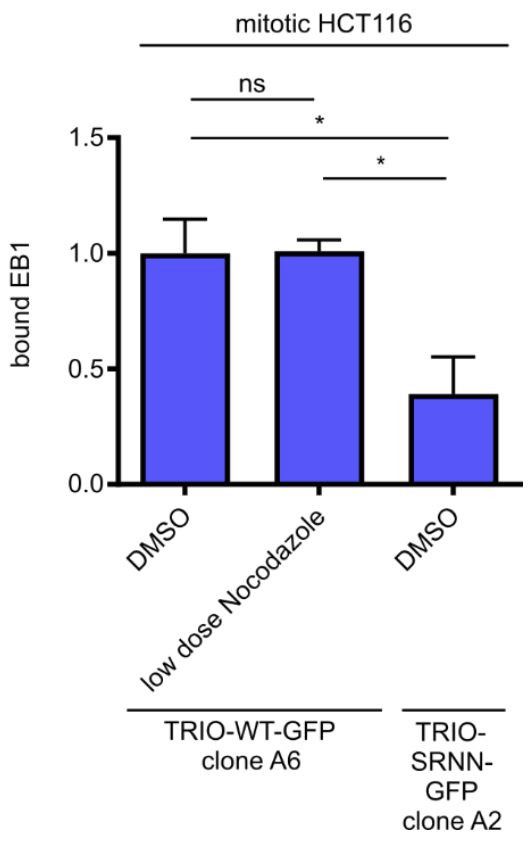

Figure 3.4: EB1-TRIO binding is not affected by a Nocodazole-induced increase of microtubule plusend assembly rates in HCT116 cells. (a) Representative Western blot of GFP pull down from mitotic HCT116 cell clones stably expressing TRIO-WT-GFP or EB1 binding deficient TRIO-SRNN-GFP reveal no impact of low dose Nocodazole treatment on the amount of EB1 bound to TRIO-WT-GFP. HCT116 clones have been generated as described before and treated with DMSO (control) or $0.5 \mathrm{nM}$ Nocodazole together with $2 \mu \mathrm{M}$ DME to induce mitotic arrest for $16 \mathrm{~h}$. Pull down of TRIO-WT-GFP or TRIO-SRNN-GFP was carried out using GFP selector beads and bound endogenous EB1 was detected on Western blots. AntiGFP and anti-EB1 antibodies were used for detection. (b) Quantification of EB1 levels from three independent GFP pull down experiments (mean \pm SEM, $t$-test). 


\subsection{The TRIO GEF-D1 activity is independent of EB1 binding}

EB1-TRIO interaction studies described in the previous chapter confirmed this binding to be SXIP motif dependent and revealed a newly identified role of microtubule dynamics in this interaction. Further experiments should now show what effect differences in mitotic EB1-TRIO binding would have downstream of this interaction, especially regarding possible actin-regulated spindle positioning. As TRIO is a well-known multidomain protein with two GEF domains, GEF-D1 for GDP/GTP exchange on Rac1 and RhoG and GEF-D2 being specific for RhoA, it plays an important role in the regulation of F-actin cytoskeleton rearrangements (Debant et al., 1996; Blangy et al., 2000). Due to the importance of the GEF function of TRIO in cytoskeletal regulation, GEF assays should reveal whether microtubule dynamics would have an effect on TRIOs GEFD1 activity via disturbance of the EB1-TRIO interaction. In order to test this, GEF assays of interphase and mitotic SW620 and HCT116 cells treated with or without low dose Taxol were performed. In this assay, the active form of Rac-GEFs was selectively pulled down from cell lysates via Rac1 G15A Agarose beads. Since this GEF assay has been shown to work out well for the GEF TIAM1 (García-Mata et al., 2006), detection of active TIAM1 was used as a control. However, while active TIAM1 was clearly detectable on Western blots of GEF assays of SW620 and HCT116 cells, TRIO was not (Figure 3.5a). Looking at the input fraction at least gave some insight into differences in expression levels between chromosomally stable HCT116 and chromosomally unstable SW620 cells as well as between mitotic and interphase cells. While HCT116 cells showed higher TIAM1 levels as well as TIAM1 GEF activity, SW620 cells showed higher TRIO expression levels. For both cell lines TIAM1 expression was higher in interphase cells, while TRIO expression levels were only higher in interphase of SW620 but not HCT116 cells when compared to mitosis. Looking at TIAM1 expression levels and TIAM1 GEF activity, these results seem to show a more important role for interphase and for chromosomally stable HCT116 cells when compared to chromosomally unstable SW620 cells. The role of TIAM1 in interphase has already been demonstrated by revealing the regulatory effect of this GEF on migration, invasion and adhesion (Minard et al., 2004). Though not having detected TRIO GEF activity levels, the observed differences in TRIO expression levels might at least serve as a hint for its higher importance for chromosomally unstable SW620 cells when compared to chromosomally stable HCT116 cells. However, its higher expression in interphase SW620 cells when compared to mitotic SW620 cells would first of all suggest a role in signaling pathways involved in interphase, which has already been verified by demonstrating the role of TRIO in migration and invasion (van Rijssel et al., 2012; Hou et al., 2018). In order to generate meaningful results, the determination of TRIO GEF-D1 activity remained critical and called for figuring out a way to perform this analysis. 
In this context, Maier et al. (2018) demonstrated TRIO to be only able to bind to wildtype Rac1, but not to Rac1 G15A, that was used for binding active GEFs within the used GEF assay due to the higher stability of this mutant compared to wildtype Rac1 (García-Mata et al., 2006). However, another group successfully showed TRIO GEF-D1 activity using Rac1 G15A coupled beads, but

a
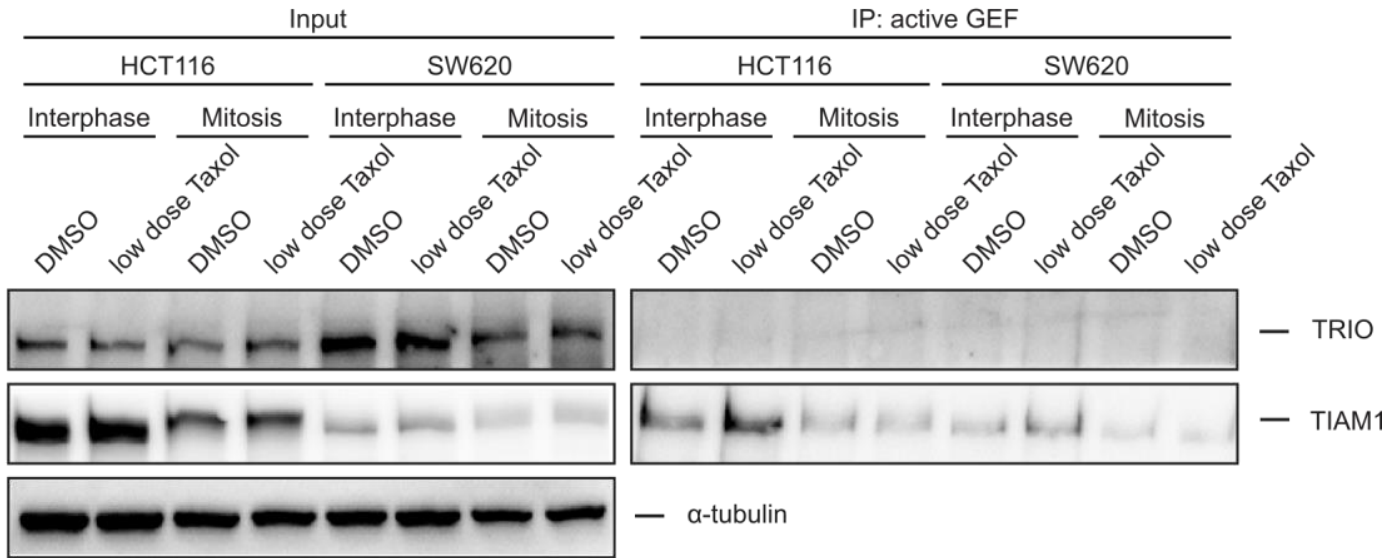

b

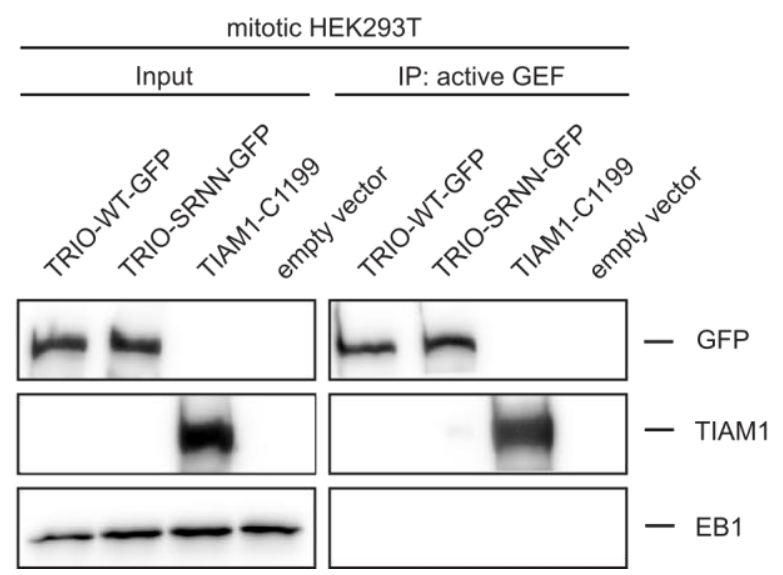

Figure 3.5: The GEF-D1 activity of TRIO is independent of EB1 binding. (a) Representative Western blot of a GEF assay from interphase and mitotic HCT116 and SW620 cells treated with or without low dose Taxol showing active endogenous TIAM1 to be detectable, but not active endogenous TRIO. For accumulating mitotic cells, $2 \mu \mathrm{M}$ DME and for low dose Taxol treatment $0.2 \mathrm{nM}$ Taxol were added $16 \mathrm{~h}$ before cell lysis. Rac1 G15A Agarose beads were used to selectively pull down the active form of Rac1GEFs from cell lysates. For detection, anti-TRIO and anti-TIAM1 antibodies were used. As loading control a-tubulin was detected. (b) Western blot of a GEF assay from mitotic HEK293T cells expressing TRIO-WTGFP, TRIO-SRNN-GFP, TIAM1-C1199 (pos. control) or an empty vector (neg. control) revealing no difference in TRIO GEF-D1 activity. HEK293T cells were transfected with indicated plasmids and treated with $2 \mu \mathrm{M}$ DME for mitotic arrest for $16 \mathrm{~h}$. GEF assay was carried out as described above. Anti-GFP and anti-TIAM1 antibodies were used for detection and anti-EB1 antibody was used to test for equal protein loading. 
only after overexpression of TRIO GEF-D1 in HeLa cells (van Rijssel et al., 2012) or in a clearly detectable manner only after increasing TRIO expression and activity by inducing inflammatory conditions via TNF- $\alpha$ treatment in endothelial HUVEC cells (van Rijssel et al., 2013). Due to these findings, further GEF assays were carried out with overexpressed TRIO, again using HEK293T cells as overexpression system. HEK293T cells were transfected with plasmids coding for TRIOWT-GFP, TRIO-SRNN-GFP, the positive control TIAM1-C1199 that is a more stable N-terminal truncated version appeared to have a higher Rac1-GEF activity than full-length TIAM1 (Michiels et al., 1995; van Leeuwen et al., 1995), or an empty vector as negative control. Using these conditions in GEF assays now allowed for detection of active TRIO in Western blots (Figure 3.5b). Quantification and comparison of active TRIO levels in TRIO-WT-GFP expressing and TRIOSRNN-GFP expressing HEK293T cells revealed no difference between the wildtype and the EB1 binding deficient mutant of TRIO in two out of four experiments. Due to a lower expression level of TRIO-SRNN-GFP than of TRIO-WT-GFP in the other two experiments, this result needs to be confirmed by repeating this experiment. However, cautiously considered this result suggests that the EB1-TRIO interaction does not affect the overall GEF-D1 activity of TRIO. This finding corresponds with work done by van Haren et al. (2014), who also observed no difference in GEFD1 activity of TRIO-WT-GFP and TRIO-SRNN-GFP by performing fluorescence-based in vitro guanine nucleotide exchange assays measuring Mant-GTP loading onto GDP-preloaded RhoG. But even if they have the same GEF activity, this does not mean that TRIO-WT-GFP and TRIOSRNN-GFP have the same effect downstream in the signaling pathway. Especially the difference in localization could alter downstream effects, as TRIO-WT-GFP but not TRIO-SRNN-GFP is able to bind to EB1 and might, thus, be rather involved in activating Rho GTPases at locations only reached by microtubule plus-tip-bound EB1.

\subsection{An increase in Rac1 activity requires EB1 binding of TRIO in mitotic cells}

Previous chapters already dealt with the impact of microtubule dynamics on the EB1-TRIO interaction and with the independency of EB1 binding on overall TRIO GEF-D1 activity. In the following, it is now important to investigate whether microtubule dynamics, EB1-TRIO binding and TRIO GEF-D1 activity affect Rac1 activity in mitotic cells. First, this was tried to be analyzed by determining endogenous Rac1 levels via pull down with PAK-PBD coated beads. Therefore, mitotic SW620 cells were treated with or without low dose Taxol, the TRIO inhibitor ITX3 or the Rac1 inhibitor NSC23766. The Rac1 activity level of mitotic HCT116 cells was also determined in order to look for general differences between these two cell lines. As positive control, the Rac1- 
GEF TIAM1-C1199 was overexpressed. However, while Tiam1-C1199 overexpression worked out well as positive control and showed the expected increase in Rac1 activity, treatments with low dose Taxol, different concentrations of the TRIO inhibitor ITX3 or with the Rac1 inhibitor NSC23766 did not show a decrease in Rac1 activity (Figure 3.6a). Maybe, this is due to the fact that Rac1 activity decreases rapidly on entry into mitosis (Yoshizaki et al., 2003), which does not allow to make a further reduction visible. Abovementioned results from GEF assays affirm a low mitotic Rac1 activity, as they show a lower amount of active TIAM1 in mitotic cells when compared to interphase cells and this is even lower for SW620 than for HCT116 cells (Figure 3.5a). Additionally, there was a lower amount of TRIO expressed in mitosis. Mitotic HCT116 cells, however, showed a higher Rac1 activity than mitotic SW620 cells (Figure 3.6a), which also goes along with the higher detected TIAM1 expression and activity in HCT116 cells (Figure 3.5a). But a higher Rac1 activity in mitotic HCT116 than in mitotic SW620 cells does not directly mean that there is no microtubule dynamic, EB1, and TRIO dependent activation of Rac1 existing in chromosomally unstable cells. It becomes clear once again, that the localization of activation of components of this pathway might be the crucial difference and not the overall activation. Thus, there might be still a part of Rac1 being activated by TRIO located at microtubule plus-tip bound EB1 that cannot be visualized under these conditions.

However, besides the low Rac1 activity in mitotic SW620 cells, the low affinity that the used PAKPBD beads also have for inactive Rac1-GDP might hide differences in active Rac1-GTP levels at these low concentrations. In order to exclude a possible effect of the Rac1 activity assay itself, activity of the Rac1 target PAK1/2 was determined as readout for Rac1 activity. PAK1/2 activity is regulated by phosphorylation at various sites, whereby binding of Rac1 or Cdc42 to the PAK-PBD domain causes autophosphorylation and conformational changes activating PAK1/2 (Knaus \& Bokoch, 1998; G. A. Martin et al., 1995). Therefore, a phospho-PAK1 (Ser199/204)/ PAK2 (Ser192/197) antibody, that has previously been used to demonstrate Rac1 dependent PAK1/2 activation (May et al., 2014; Whalley et al., 2015), was used to detect active PAK1/2. Again, mitotic SW620 were treated with DMSO, low dose Taxol, the TRIO inhibitor ITX3 or the Rac1 inhibitor NSC23766 and compared with DMSO treated mitotic HCT116 cells. This time, there was a significant and concentration dependent reduction of phospho-PAK1/2 levels visible after treatment of SW620 cells with ITX3 or NSC23766 indicating a reduced Rac1 activity (Figure 3.6b). This is not surprising, as TRIO is a well-known GEF for Rac1 and, thus, TRIO inhibition is expected to reduce Rac1 activity. However, when having a look at the effect of low dose Taxol treatment, there is no change in phospho-PAK1/2 levels of mitotic SW620 cells. This result demonstrates for SW620 cells that a Taxol-induced rescue of increased microtubule plus-end assembly rates does 
Results

a

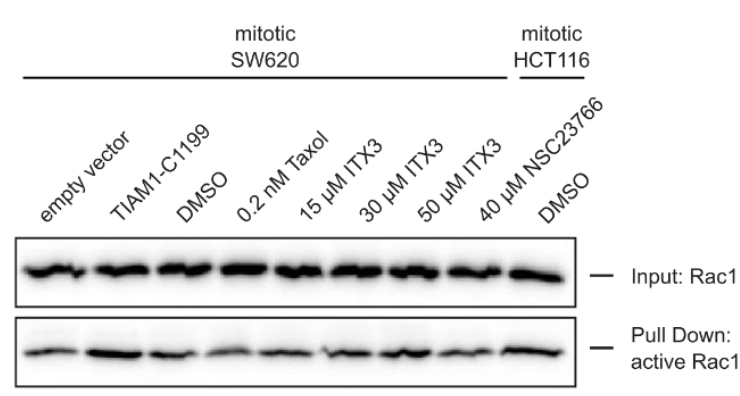

b

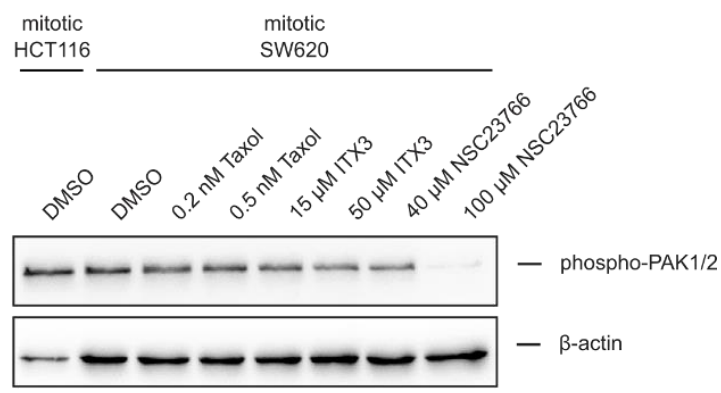

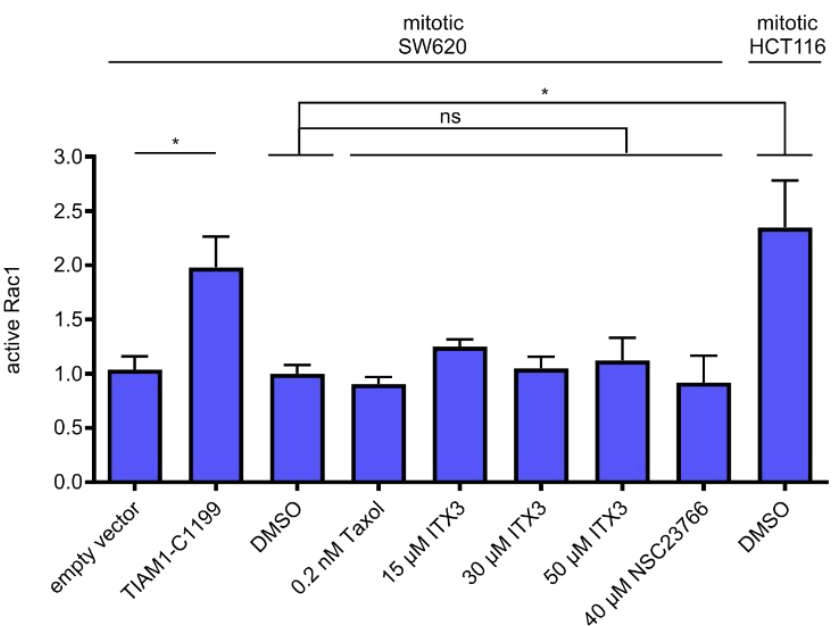

mitotic

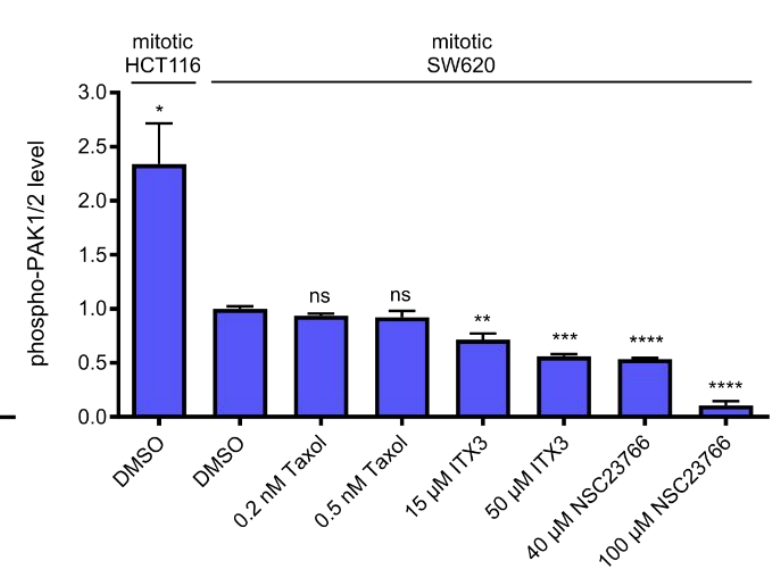

Figure 3.6: Rac1 activity is reduced by TRIO inhibition, but not by a Taxol-induced decrease of microtubule plus-end assembly rates. (a) Representative Western blot of a Rac1 activity assay from mitotic SW620 and HCT116 cells treated with or without low dose Taxol, ITX3 (TRIO inhibitor) or NSC23766 (Rac1 inhibitor). SW620 cells were transfected with a TIAM1-C1199 expressing plasmid (pos. control) or an empty vector or treated with DMSO (control), $0.2 \mathrm{nM}$ Taxol, $15 \mu \mathrm{M}, 30 \mu \mathrm{M}$ or $50 \mu \mathrm{M}$ ITX3 or $40 \mu \mathrm{M}$ NSC23766 for $16 \mathrm{~h}$. To induce mitotic arrest, $2 \mu \mathrm{M}$ DME was added $16 \mathrm{~h}$ before cell lysis. Rac1 activity assays were performed using PAK-PBD beads for pull down of active Rac1 from cell lysates. For detection, an anti-Rac1 antibody was used. Bar graphs show quantification of active Rac1 from three or two (15 and $30 \mu \mathrm{M}$ ITX3) independent Rac1 activity assays (mean \pm SEM, $t$-test). (b) Representative Western blots of phospho-PAK1/2 levels from mitotic SW620 and HCT116 cells after treatment with or without low dose Taxol, TRIO inhibitor or Rac1 inhibitor. Cells have been treated with DMSO (control), $0.2 \mathrm{nM}$ Taxol, $15 \mu \mathrm{M}$ or $50 \mu \mathrm{M}$ ITX3, $40 \mu \mathrm{M}$ or $100 \mu \mathrm{M}$ NSC23766 together with $2 \mu \mathrm{M}$ DME for $16 \mathrm{~h}$. Active PAK1/2 as readout for active Rac1 was detected on Western blots by a phospho-PAK1 (Ser199/204)/ PAK2 (Ser192/197) antibody and detection of $\beta$-actin served as loading control. Data was normalized to DMSO treated SW620 cells. Bar graphs show quantification of phospho-PAK $1 / 2$ levels from two to three independent experiments (mean \pm SEM, $t$-test).

not have an impact on overall Rac1 activity. However, this again might be explained by the importance of a microtubule plus-tip, EB1, TRIO dependent localization of activation of maybe only a small part of Rac1. While inhibitors as ITX3 for TRIO and NSC23766 for Rac1 affect the whole pool of active Rac1 within a cell, microtubule dynamic changes by low dose Taxol would only affect Rac1 activity that is associated with TRIO bound to EB1 at microtubule plus-tips, which 
might not be detectable within the performed assays. But maybe it is exactly that part of Rac1 that is involved in actin dependent cytoskeletal changes important for the regulation of spindle positioning.

Since the detection of phospho-PAK1/2 levels could not reveal whether there is a part of Rac1 being activated via a microtubule plus-tip, EB1, TRIO dependent pathway, again HEK293T cells were used for expressing TRIO-WT-GFP or TRIO-SRNN-GFP in order to look for a possible difference in Rac1 activation. Although TRIO-WT-GFP and TRIO-SRNN-GFP has been shown to have the same GEF-D1 activity (chapter 3.2), there might nevertheless be differences in their activation of downstream targets caused by differences in their localization and, thus, site of action. Therefore, Rac1 activity assays were performed with mitotic HEK293T cells transfected with plasmids encoding TRIO-WT-GFP, TRIO-SRNN-GFP, TIAM1-C1199 as positive control or an empty vector. While cells expressing the Rac1-GEF TIAM1-C1199 showed an almost 3-fold increase in Rac1 activity, the TRIO variants increased Rac1 activity to a lesser extend (Figure 3.7a). However, TRIO-WT-GFP expression resulted in a $39 \%$ increase in Rac1 activity, whereas the EB1 binding deficient TRIO-SRNN-GFP only lead to a small increase in Rac1 activity of about $18 \%$. These results reveal the importance of EB1-TRIO binding for the downstream activation of Rac1. To prove these results, Rac1 assays were repeated with HCT116 cells that have been transfected with plasmids coding for GFP, TRIO-WT-GFP or TRIO-SRNN-GFP. While TRIO-WTGFP expression increased Rac1 activity by almost 2-fold, TRIO-SRNN-GFP led only to a Rac1 activity increase of about $63 \%$ (Figure 3.7b). Taken together, the results show that transient overexpression of both TRIO-SRNN-GFP and TRIO-WT-GFP causes increased Rac1 activity, but that this increase is higher for TRIO that still allows for EB1 binding. Thus, there seems to be a Rac1-activating pathway dependent on TRIO bound to EB1 at microtubule plus-tips.

As the generation of HCT116 cell clones stably expressing EGFP, TRIO-WT-GFP or TRIO-SRNNGFP was finally successful, they were used for further Rac1 activity assays in order to test, whether an ongoing overexpression of TRIO-WT-GFP or TRIO-SRNN-GFP would have an additional impact on the cells Rac1 activity. Surprisingly, the EB1 binding deficient mutant of TRIO did not show any increase in Rac1 activity any more, while the EB1 binding wildtype of TRIO still exhibited approximately 2- to 3-fold activation of Rac1 dependent on the clone tested (Figure 3.8ab). Looking for an explanation for this difference between transiently and stably overexpressed TRIO-SRNN-GFP, consequences due to differences in the expression levels of individual cells could be considered. A transient transfection usually leads to a mixed cell population, of which 
Results
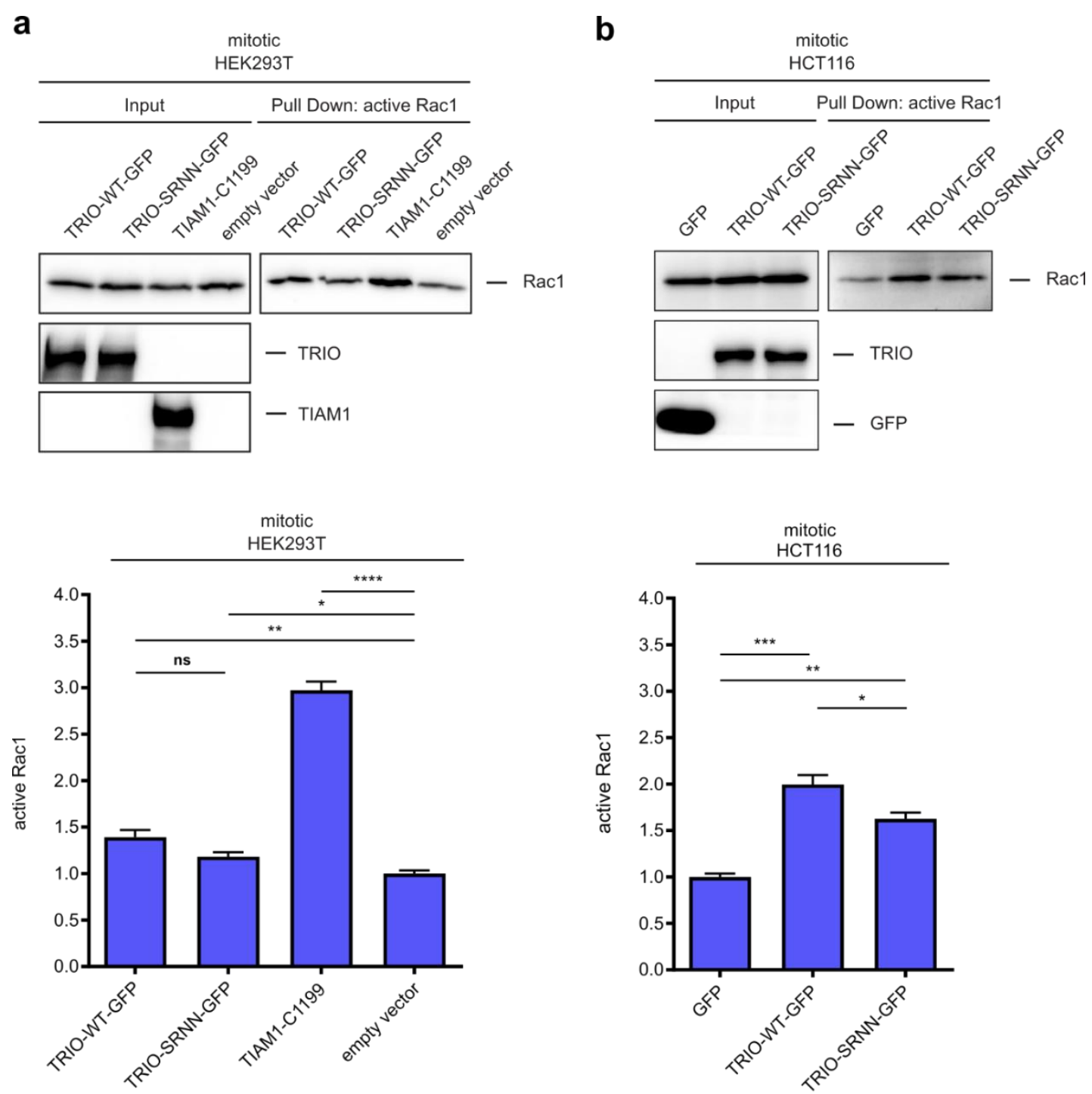

Figure 3.7: EB1 binding of TRIO is needed for a stronger increase in Rac1 activity of mitotic cells. Representative Western blots of Rac1 activity assays from mitotic (a) HEK293T or (b) HCT116 cells showing a higher increase in Rac1 activity when expressing the wildtype of TRIO compared to the EB1 binding deficient mutant of TRIO. HEK293T cells were transfected with TRIO-WT-GFP, TRIO-SRNN-GFP, TIAM-C1199 encoding plasmids or an empty vector and HCT116 cells were transfected with plasmids expressing GFP, TRIO-WT-GFP or TRIO-SRNN-GFP. Both cell lines were treated with $2 \mu \mathrm{M}$ DME $16 \mathrm{~h}$ before cell lysis. Rac1 activity assays were carried out as described above. Anti-Rac1, anti-GFP and antiTIAM1 antibodies were used for detection. Bar graphs show quantification of three independent Rac1 activity assays (mean \pm SEM, $t$-test).

some cells have not been transfected, others express the protein in a low to moderate manner and still others show a huge overexpression, mainly due to a different copy number of the transfected plasmid. In contrast, the generated cell clones consist of a cell population with rather equal and moderate protein expression, although TRIO levels were still much higher than in GFPexpressing control clones (Figure 3.3). As consequence, an increase in Rac1 activity in transiently transfected cells might be only due to the existence of a part of the cell population showing a huge 
overexpression of TRIO-SRNN-GFP, thereby hiding that a moderate overexpression is not sufficient to increase Rac1 activity. Consequently, this would also mean that the wildtype of TRIO leads to increased Rac1 activity not only when strongly but also when moderately overexpressed.

Summed up, the abovementioned results clearly show that EB1 binding of TRIO is crucial for Rac1 activation in mitotic cells by that supporting the hypothesis of a mitotic microtubule plus-tip, EB1, TRIO dependent pathway for Rac1 activation.

a

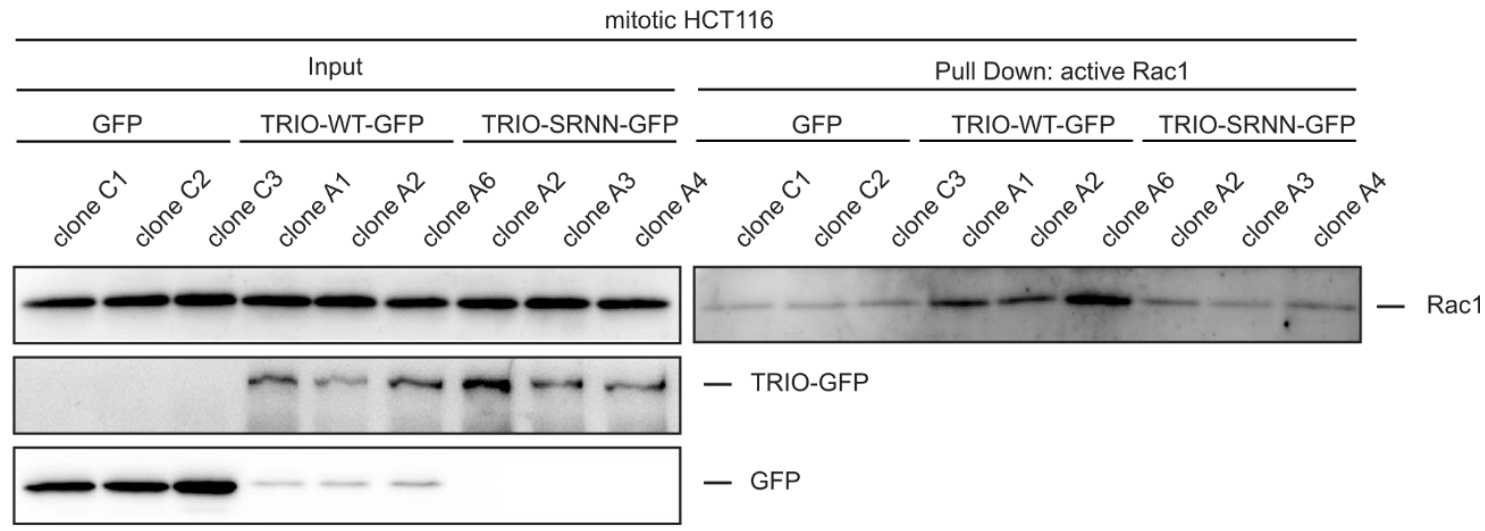

b

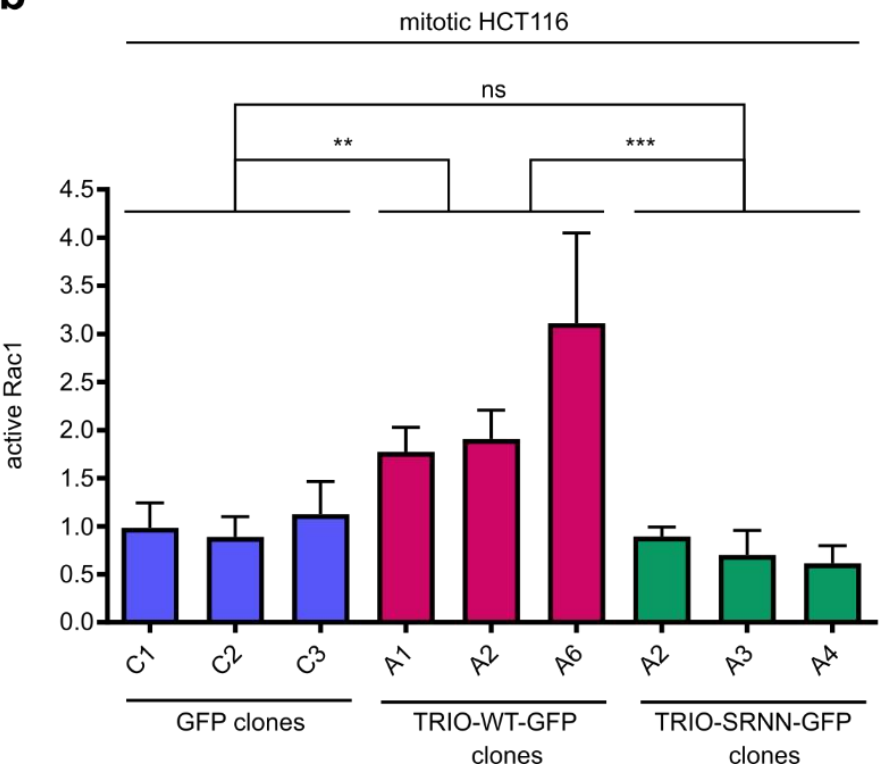

Figure 3.8: EB1-TRIO binding is crucial for activating Rac1 in mitotic cells with moderate, long-term TRIO overexpression. (a) Representative Western blot of a Rac1 assay from mitotic HCT116 cell clones stably expressing GFP, TRIO-WT-GFP or TRIO-SRNN-GFP demonstrating that EB1 binding of TRIO is needed for Rac1 activation in mitotic cells. HCT116 clones were generated and Rac1 activity assays were performed as described before. Cells were treated with $2 \mu \mathrm{M}$ DME for $16 \mathrm{~h}$. Rac1 was detected using an anti-Rac1 antibody and for detection of TRIO-WT-GFP, TRIO-SRNN-GFP as well as GFP an anti-GFP antibody was used. (b) Quantification of active Rac1 levels from three independent Rac1 activity assays (mean \pm SEM, $t$-test). 


\subsection{TRIO overexpression increases cell migration to a higher extend when EB1 binding is disturbed}

Live cell imaging of HCT116 cell clones showed a clear localization of TRIO-WT-GFP and TRIOSRNN-GFP at lamellipodia-like structures of interphase cells with lamellipodia being even larger than in control cells (Figure 3.3), which confirms TRIOs function in lamellipodia formation and migration described by van Rijssel et al. (2012). This finding, together with the observation that TRIO-SRNN-GFP localization at lamellipodia was even more pronounced than TRIO-WT-GFP localization (Figure 3.3), is in contradiction with the lack of Rac1 activation seen for TRIO-SRNNGFP in the Rac1 activity assays described above. In order to prove the actual impact of overexpressed TRIO-WT-GFP and TRIO-SRNN-GFP on the migrative behavior of cells, HCT116 cell clones were used for migration assays. In these assays, FCS-starved cells were seeded into cell culture inserts with $8 \mu \mathrm{m}$ wide pores surrounded by FCS-containing medium. Cells that have been migrated through the pores within $24 \mathrm{~h}$ were detached and counted. As expected, overexpression of TRIO leads to an increase in cell migration (Figure 3.9a). TRIO-SRNN-GFP cell clones even showed a higher migration rate than TRIO-WT-GFP cell clones, thereby supporting the observation of a more pronounced localization of TRIO-SRNN-GFP at lamellipodia. This result again contradicts the Rac1 activity assay results, since Rac1 has been reported as important factor for lamellipodium-driven cell migration (Steffen et al., 2013). However, Rac1 activity assays have only been carried out for mitotic HCT116 cell clones so far and needed to be repeated with interphase cells in order to actually prove the role of both TRIO variants in interphase-related cell migration. This time, Rac1 activity assays revealed an increase in Rac1 activity for both TRIOWT-GFP and TRIO-SRNN-GFP overexpressing cells (Figure 3.9b-c). Although it was only a slight increase of $20 \%$ to $55 \%$ for TRIO-SRNN-GFP cell clones, it was a clear difference when compared to mitotic cells. Interphase TRIO-WT-GFP overexpressing cell clones showed a stronger increase in Rac1 activity of $58 \%$ to $124 \%$, even if it was not as pronounced as for mitotic cells $(77 \%-211 \%$; Figure $3.8 \mathrm{a}-\mathrm{b})$. This result suggests that EB1 binding of TRIO is more important for local Rac1 activation in mitotic cells than in interphase cells, where Rac1 also becomes activated by EB1 binding deficient TRIO. 
a

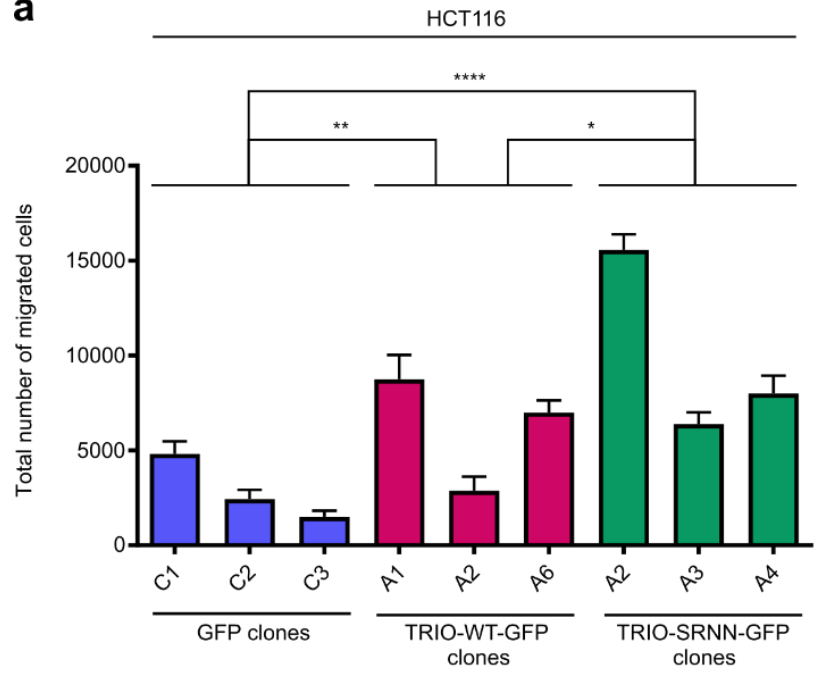

C

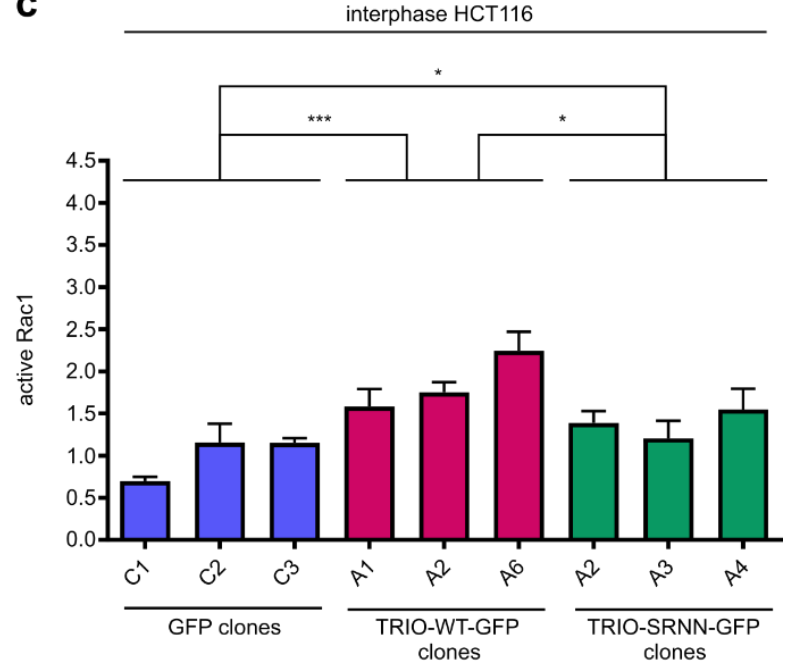

b

interphase HCT116

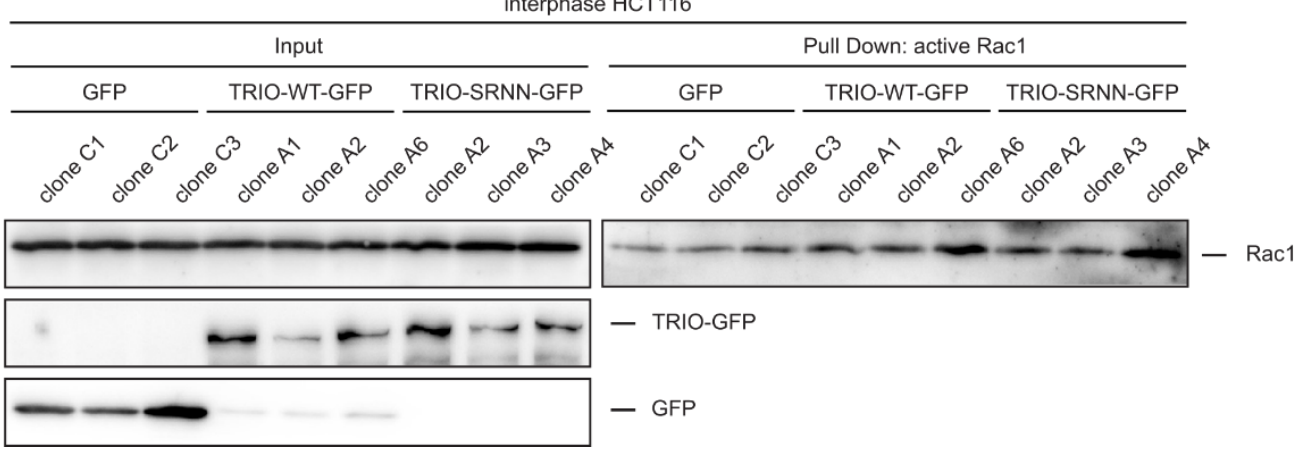

Figure 3.9: Overexpressed TRIO induces increased cell migration to a greater extend with EB1 binding deficiency. (a) Transwell migration assays of HCT116 cell clones expressing GFP, TRIO-WT-GFP or TRIO-SRNN-GFP showing a stronger increase in cell migration for EB1 binding deficient TRIO. HCT116 were generated as described previously. 200,000 FCS-starved cells were seeded into $8 \mu \mathrm{m}$ pore-containing cell culture inserts surrounded by FCS-containing medium for $24 \mathrm{~h}$. Migrated cells were detached and counted. Bar graphs show the proportion of migrated cells from four independent migration assays (mean \pm SEM, $t$-test). (b) Representative Western blot of a Rac1 assay from interphase HCT116 cell clones stably expressing GFP, TRIO-WT-GFP or TRIO-SRNN-GFP showing that also EB1 binding deficient TRIO induces Rac1 activation in interphase cells. Rac1 activity assays were performed as described before. For detection of Rac1, an anti-Rac1 antibody was used and TRIO-WT-GFP, TRIO-SRNN-GFP as well as GFP were detected using an anti-GFP antibody. (c) Quantification of active Rac1 levels from three independent Rac1 activity assays (mean \pm SEM, $t$-test). 


\subsection{Overexpression of TRIO induces spindle axis misalignment, chromosome mis- segregation and chromosomal instability in an EB1 binding dependent manner}

Abovementioned results already revealed that EB1 binding of TRIO is affected by microtubule dynamics and that it is crucial for mitotic Rac1 activation. However, it still needed to be figured out whether the pathway revealed so far was actually involved in the induction of spindle axis misalignment and increased amounts of cells exhibiting lagging chromosomes, which has been shown to result in chromosomal instability (Ertych et al., 2014). In order to investigate possible effects on spindle positioning, the spindle axis angles of the generated HCT116 cell clones expressing GFP, TRIO-WT-GFP or TRIO-SRNN-GFP were determined. In fact, HCT116 cell clones expressing wildtype TRIO showed a significant increase of the spindle axis angle (between $15.44^{\circ}$ and $16.51^{\circ}$ ) when comparted to GFP (between $10.11^{\circ}$ and $11.05^{\circ}$ ) or TRIO-SRNN-GFP (between $8.96^{\circ}$ and $9.29^{\circ}$ ) expressing cells (Figure $3.10 \mathrm{a}$ and $\mathrm{b}$ ). This finding confirms the results from Schermuly (2019), showing transient expression of TRIO-WT-GFP, but not TRIO-SRNNGFP to induce spindle axis misalignment in HCT116 cells. Furthermore, this result is in line with the observed differences in mitotic Rac1 activation of these clones (Figure 3.8), thereby proving the importance of EB1 binding of TRIO and of the resulting increase in Rac1 activity for the induction of spindle axis misalignment.

Previous work from our group showed that an increase in prometaphase spindle axis misalignment results in an increase in chromosome mis-segregation determined by a larger proportion of cells exhibiting lagging chromosomes (Ertych et al., 2014). In order to prove whether the increased spindle axis misalignment in HCT116 cell clones would also have an impact on chromosome segregation, the amount of cells with lagging chromosomes was determined. Therefore, HCT116 cell clones underwent a double thymidine block in order to synchronize cells at G1/S phase. $8.5 \mathrm{~h}$ after the second release cells were fixed, as then a large part of cells was in anaphase. Microtubules, centromeres and DNA was stained with antibodies against $\alpha$-tubulin, CENP-C and Hoechst, respectively. Only chromosomes positive for CENP-C and Hoechst that were located clearly separated in between two pole-oriented chromosome masses were counted as lagging chromosomes. Again, only HCT116 cell clones expressing wildtype TRIO showed an effect by increasing the amount of cells with lagging chromosomes to up to $10 \%$, while GFP expressing clones had at most $3.67 \%$ and TRIO-SRNN-GFP clones $4.33 \%$ of lagging chromosomes (Figure $3.10 \mathrm{c}$ and d). This effect has also been seen in HCT116 cells transiently expressing TRIO-WTGFP or TRIO-SRNN-GFP by Schermuly (2019). Since chromosomal instability is defined as an 
Results

a

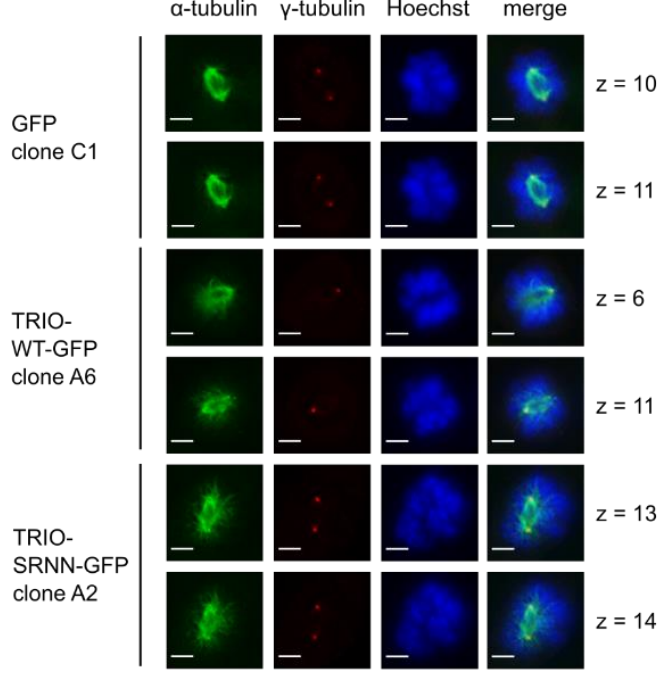

C

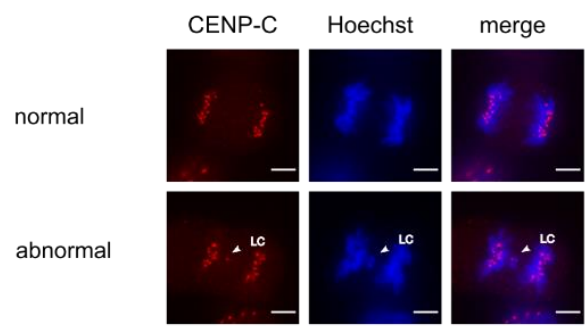

b

mitotic HCT116

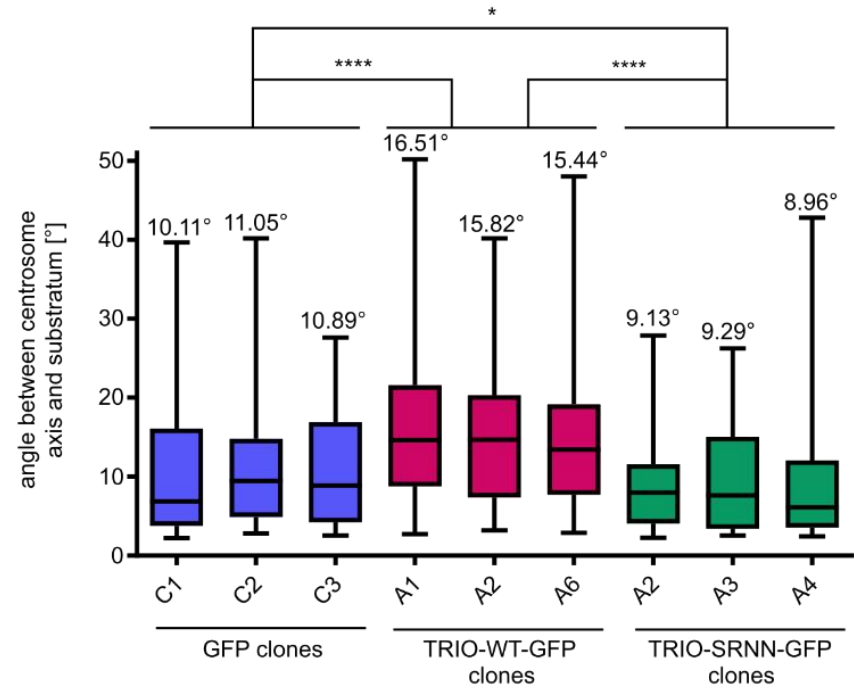

d

mitotic HCT116

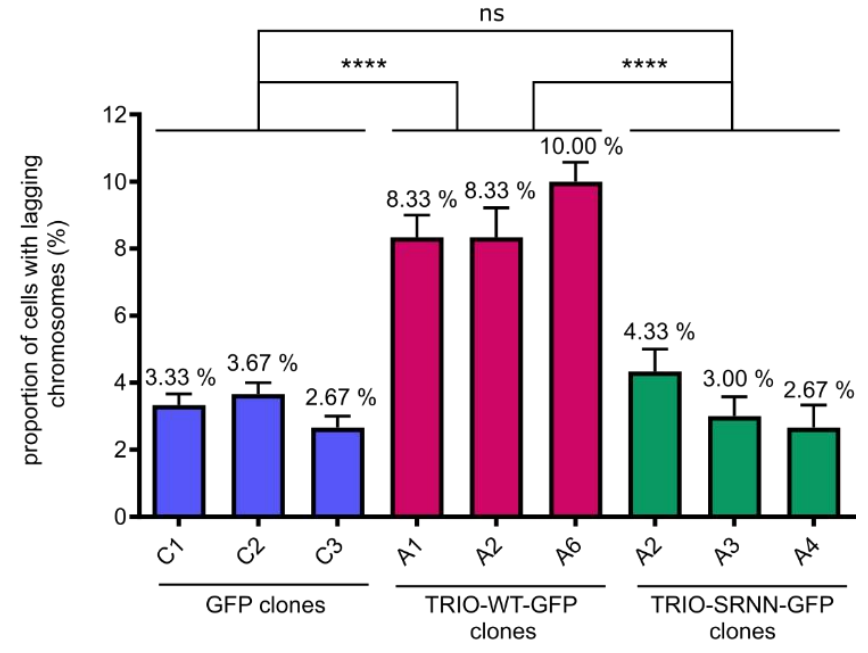

e mitotic HCT116

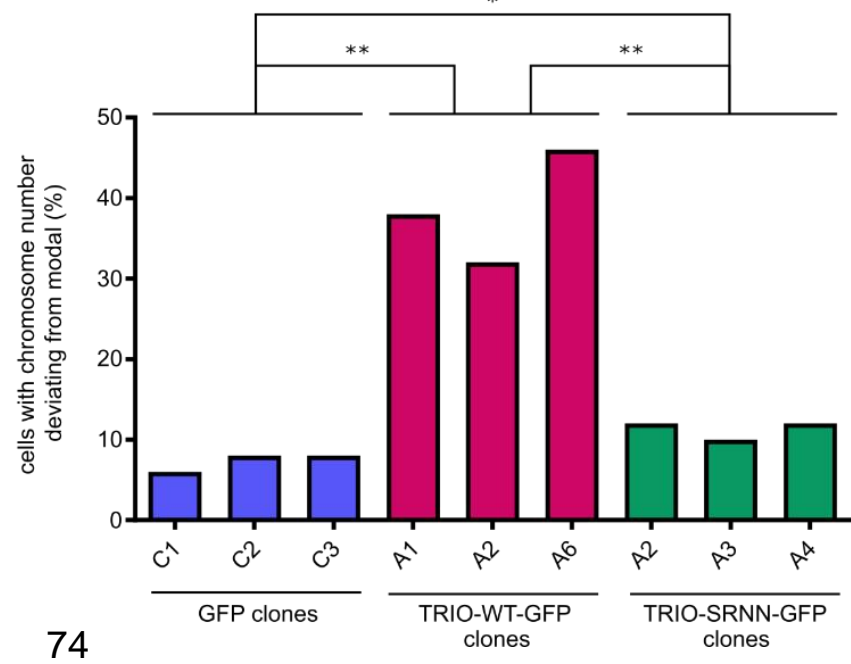


Figure 3.10: TRIO overexpression leads to an EB1 binding dependent increase in spindle axis misalignment, in the amount of lagging chromosomes and in karyotype variability. (a) Representative immunofluorescence images of prometaphase cells showing only an increase in spindle axis angle for wildtype TRIO but not EB1 binding deficient TRIO expressing HCT116 cell clones. HCT116 cell clones expressing GFP, TRIO-WT-GFP or TRIO-SRNN-GFP were generated as described previously, seeded onto fibronectin-coated glass coverslips and stained for $\alpha$-tubulin (microtubules; green), $\gamma$-tubulin (centrosomes; red) and Hoechst33342 (DNA; blue). Scale bar $5 \mu \mathrm{m}$. (b) Box and whisker plots of spindle axis angle measurements of cells depicted in (a) showing median, quartile and range of three independent experiments ( $\mathrm{n}=20$ cells per experiment, $t$-test). (c) Representative immunofluorescence images of anaphase HCT116 cells with (normal) and without (abnormal) the occurrence of a lagging chromosome (LC). Cells were synchronized by a double thymidine block and fixed after release for $8.5 \mathrm{~h}$ to obtain an increased amount of anaphase cells. Cells were stained for a-tubulin, CENP-C (centromeres; red) and Hoechst33342. Scale bar $5 \mu \mathrm{m}$. (d) The amount of lagging chromosomes is increased in HCT116 cell clones expressing wildtype TRIO, but not EB1 binding deficient TRIO. HCT116 cell clones were synchronized and stained as described in (c). For determination of lagging chromosomes in anaphase cells, only chromosomes positive for Hoechst and CENP-C that were located clearly separated in between two poleoriented chromosome masses were counted. Bar graphs show the proportion of cells with lagging chromosomes from three independent experiments $(n=100$ analyzed anaphase cells per experiment, mean \pm SEM, $t$-test). (e) Karyotype analysis of HCT116 cell clones showing chromosomal instability for TRIO-WTGFP, but not for TRIO-SRNN-GFP expressing cell clones. HCT116 cell clones have been cultivated for approximately 30 generations after single cell isolation and used for preparing metaphase chromosome spreads. Bar graphs show the proportion of cells with a chromosome number deviating from the modal number ( $n=50$ metaphase spreads for each clone were counted).

increased rate of chromosome mis-segregation (Thompson et al., 2010), these results claimed the wildtype TRIO expressing HCT116 cell clones to be chromosomally unstable. To prove this, chromosomes of metaphase spreads of HCT116 cell clones, which have been cultivated for about 30 generations after single cell isolation, were counted for karyotype analysis. In fact, in HCT116 cell clones expressing TRIO-WT-GFP $32 \%$ to up to $46 \%$ of cells exhibited a chromosome number that deviated from the modal number (Figure 3.10 e). In contrast, GFP expressing clones showed with $6 \%$ to $8 \%$ and TRIO-SRNN-GFP expressing clones with $10 \%$ to $12 \%$ quite a stable karyotype. These findings further confirmed the role of the hypothesized microtubule plus-tip, EB1, TRIO, Rac1 dependent pathway in the induction of chromosomal instability. 


\subsection{Chromosomal instability induced by overexpression of TRIO is rescuable by inhibition of TRIO GEF-D1, Rac1 or Arp2/3}

So far it could be shown that overexpression of wildtype TRIO increases mitotic Rac1 activity in an EB1 binding dependent manner and triggers chromosomal instability via the induction of spindle axis misalignment. Further work should show whether Rac1 is the only target of TRIO responsible for the generation of chromosomal instability. As TRIO has not only GEF function for Rac1, but also for RhoG and RhoA, which activity changes due to TRIO overexpression had not been analyzed, it was important to clarify the components downstream of TRIO in the suggested pathway. This also included proof of the hypothesized link between a TRIO-induced increased mitotic Rac1 activity and the activation of the actin cytoskeleton regulator Arp2/3 within this pathway, as spindle positioning has been shown to be regulated by mitotic Arp2/3 dependent subcortical actin clouds (Mitsushima et al., 2010; Kwon et al., 2015). For this purpose, generated HCT116 cell clones expressing GFP, TRIO-WT-GFP or TRIO-SRNN-GFP were treated with low dose Taxol, low dose Nocodazole, the TRIO inhibitor ITX3, the Rac1 inhibitor NSC23766 or the Arp2/3 inhibitor CK666, followed by analyzing their effect on chromosome mis-segregation by counting lagging chromosomes. Additionally, single cell subclones were isolated from HCT116 cell clones and treated with the abovementioned drugs for further 30 generations, followed by preparing and counting metaphase chromosome spreads for karyotype analysis. Low dose Taxol treatment was initially performed as negative control, since HCT116 cell clones have been shown to exhibit normal microtubule plus-end assembly rates that were hardy affected by subnanomolar concentrations of Taxol (Ertych et al., 2014). Therefore, an interference within this pathway upstream of TRIO was thought to have no impact on the downstream effect of a TRIO overexpression. Low dose Nocodazole was used to induce increased microtubule plus-end assembly rates, thereby generating a phenotype given in chromosomally unstable cells (Ertych et al., 2014). Initially, the idea was that generating an environment with increased microtubule plusend assembly rates and overexpressed TRIO in chromosomally stable HCT116 cells might cause an even greater increase in chromosomal instability than TRIO overexpression alone. However, as low dose Nocodazole treatment had not shown any impact on EB1-TRIO binding (Figure 3.4), it was uncertain what effect it would have on chromosomal instability of the analyzed clones. The inhibitor ITX3 only inhibits the TRIO GEF-D1 domain, but not the GEF-D2 domain (Bouquier et al., 2009). Using this inhibitor allowed to unravel possible effects of TRIO GEF-D2-activated RhoA on chromosomal instability, as these effects would continue in ITX3 treated clones. For Rac1 inhibition, the widely used Rac1 inhibitor NSC23766 was used, thereby proving whether Rac1 was the actual TRIO target responsible for the induction of chromosomal instability. Finally, using the 
Arp2/3 inhibitor CK666 should help to figure out, whether the F-actin polymerization promoter Arp2/3 was the actual Rac1 target involved in the described chromosomal instability-inducing pathway. In order to investigate possible effects of the abovementioned treatments on chromosomal instability, chromosome mis-segregation rates were determined by counting anaphase lagging chromosomes of HCT116 cell clones expressing GFP, TRIO-WT-GFP or TRIOSRNN-GFP. The results confirmed the finding illustrated in $d$, showing that only overexpression of wildtype TRIO increased the amount of cells with lagging chromosomes (Figure 3.11a). This demonstrates once more that EB1 binding is required for TRIO to induce chromosome missegregation. Treatment with the abovementioned drugs did not have an impact on the generation of lagging chromosomes in GFP or TRIO-SRNN-GFP expressing clones (Figure 3.11b). Only Nocodazole treatment of GFP expressing cells induced a slight increase that has already been observed in previous studies from our group (Berger, 2016; Ertych et al., 2014; Schermuly, 2019). However, Nocodazole also did not affect the generation of lagging chromosomes in TRIO-WTGFP and TRIO-SRNN-GFP expressing clones. This result further indicates that the microtubuleaffecting impact of Nocodazole might be somehow restricted by TRIO overexpression in HCT116 cells, thus, supporting the finding from EB1-TRIO binding studies (Figure 3.4). Moreover, it could also play a role that TRIO overexpression alone already results in a higher amount of TRIO interacting with EB1. This can be explained by assuming EB1-TRIO binding follows a saturation curve with other EB1 binding proteins acting as competitors as described by Michaelis-Menten kinetics (Michaelis \& Menten, 1913). Consequently, a high abundancy of TRIO in the cell would already mean a higher amount of wildtype TRIO bound to EB1 with or without a Nocodazoleinduced increase in microtubule plus-end assembly rates. Surprisingly, low dose Taxol treatment of TRIO-WT-GFP clones showed a rescue of the increased generation of lagging chromosomes (Figure 3.11b). While TRIO overexpression was shown to have no impact on microtubule plusend assembly rates that could have been an explanation for this Taxol-induced rescue (Figure 3.3c), the disturbed EB1-TRIO binding due to Taxol treatment that have been detected in GFP pull down assays might rather be the cause (Figure 3.2a and b). Following Michaelis-Menten kinetics once more, the increased amount of TRIO bound to EB1 as consequence of its high abundancy due to being overexpressed might be decreased again by a Taxol-induced disturbance of the EB1-TRIO interaction. Consequently, the pathway downstream of EB1-TRIO interaction would be less activated resulting in the observed rescue of lagging chromosome formation. Having a look at the result of the other treatments in TRIO-WT-GFP expressing clones, inhibition of TRIO GEF-D1, Rac1 and also Arp2/3 showed a rescue of chromosome mis-segregation 
Results

a

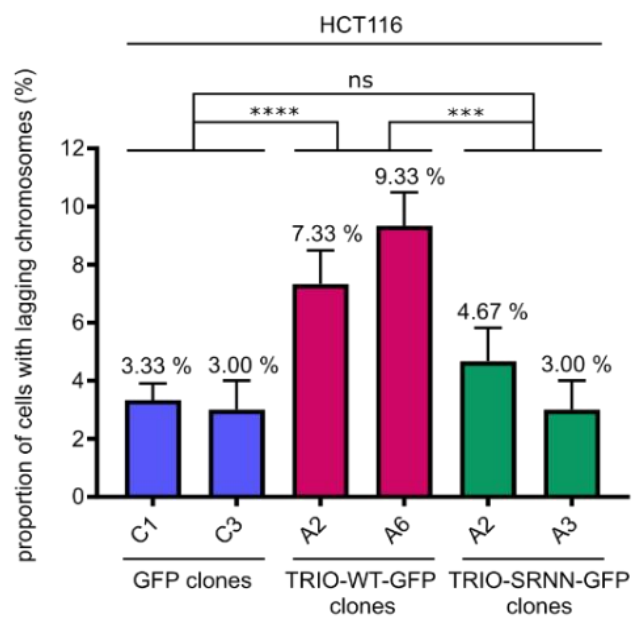

C

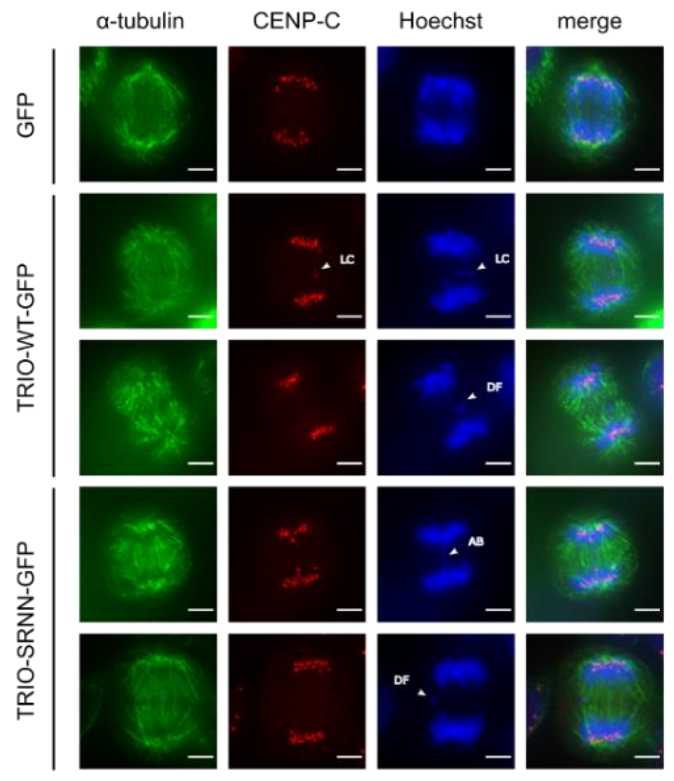

b
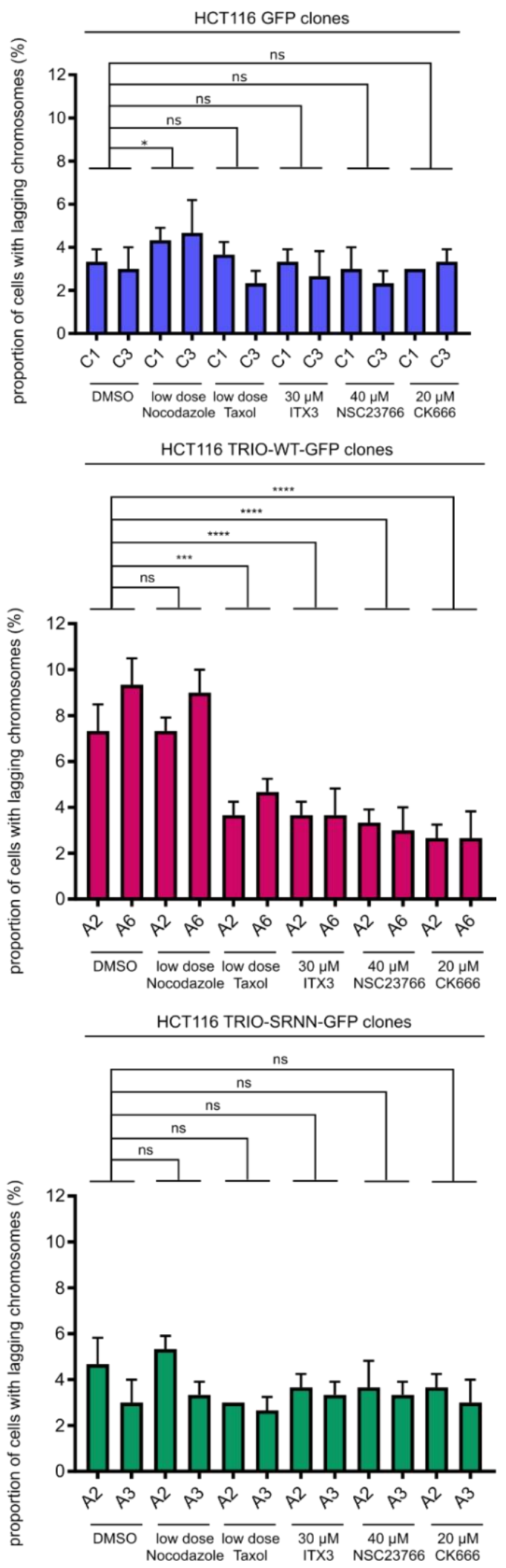
Figure 3.11: The increase in lagging chromosome formation in TRIO overexpressing HCT116 cells can be rescued by low dose Taxol as well as inhibition of TRIO GEF-D1, Rac1 or Arp2/3. (a) HCT116 cell clones expressing wildtype TRIO, but not EB1 binding deficient TRIO show an increase in the generation of lagging chromosomes. HCT116 cell clones expressing GFP, TRIO-WT-GFP or TRIO-SRNN-GFP were generated as described above and synchronized by a double thymidine block with fixation $8.5 \mathrm{~h}$ after release (DMSO control in b). Cells were stained for $\alpha$-tubulin, CENP-C and Hoechst33342. Lagging chromosomes were determined as previously described. Bar graphs show the proportion of cells with lagging chromosomes from three independent experiments $(n=100$ analyzed anaphase cells per experiment, mean \pm SEM, $t$-test). (b) Increased lagging chromosome generation induced by wildtype TRIO overexpression can be rescued by low dose Taxol or inhibition of TRIO GEF-D1, Rac1 or Arp2/3. HCT116 cell clones were synchronized as described above and treated with DMSO (control), $0.5 \mathrm{nM}$ Nocodazole, $0.5 \mathrm{nM}$ Taxol, $30 \mu \mathrm{M}$ ITX3, $40 \mu \mathrm{M}$ NSC23766 or $20 \mu \mathrm{M}$ CK666 for $8 \mathrm{~h}$. Cells were stained and examined for lagging chromosomes as described above. Bar graphs show the proportion of cells with lagging chromosomes from three independent experiments ( $n=100$ analyzed anaphase cells per experiment, mean \pm SEM, $t$-test). (c) Representative immunofluorescence images of anaphase HCT116 cell clones from (a) showing the occurrence of lagging chromosomes (LC), DNA fragments (DF) or anaphase bridges (AB). Scale bar $5 \mu \mathrm{m}$.

(Figure 3.11b). This confirms the introduced pathway, as it excludes other TRIO targets than Rac1 from being involved in the induction of chromosomal instability and proves Arp2/3 as missing link between the upstream pathway and the F-actin cytoskeleton.

However, during counting lagging chromosomes other structural abnormalities than lagging chromosomes were observed in TRIO expressing clones. These especially included anaphase bridges and DNA fragments located separately from the two-pole oriented chromosome masses of anaphase cells that had no CENP-C (centromere) signal (Figure 3.11c). While an increase in the amount of anaphase bridges was strong in TRIO-WT-GFP and only slight in TRIO-SRNN-GFP expressing clones (Figure 3.12a), the increase in the number of DNA fragments was similar for TRIO-WT-GFP and TRIO-SRNN-GFP clones (Figure 3.12c). Interestingly, anaphase bridges in TRIO-WT-GFP clones could be rescued to the level of untreated TRIO-SRNN-GFP clones by treatment with low dose Taxol or the inhibitors against TRIO, Rac1 and Arp2/3 (Figure 3.12b). The rescue was even stronger for TRIO-WT-GFP clone A2 than for clone A6 with clone A2 showing the lesser TRIO overexpression (Figure 3.3a). However, there was no further decrease detectable for TRIO-SRNN-GFP clones after inhibitor treatment, resulting in a similar slight increase of anaphase bridges in both TRIO-WT-GFP and TRIO-SRNN-GFP clones when compared to control GFP clones. This result suggests that the occurrence of anaphase bridges can only partially be caused by the given pathway. Instead, EB1 binding independent TRIO targets seem to play a role in the induction of anaphase bridge formation. When looking at the amount of DNA fragments, there was also a slight rescue for TRIO-WT-GFP clones after low dose Taxol treatment or inhibition of TRIO, Rac1 or Arp2/3 (Figure 3.12d). Again, the rescue was more pronounced in the TRIO-WT-GFP clone A2 expressing less TRIO than clone A6. In contrast, TRIO-SRNN-GFP clones did not show a significant reduction of the amount of DNA fragments. These results confirm the abovementioned suggestion of the existence of another pathway independent of EB1 bound 
Results
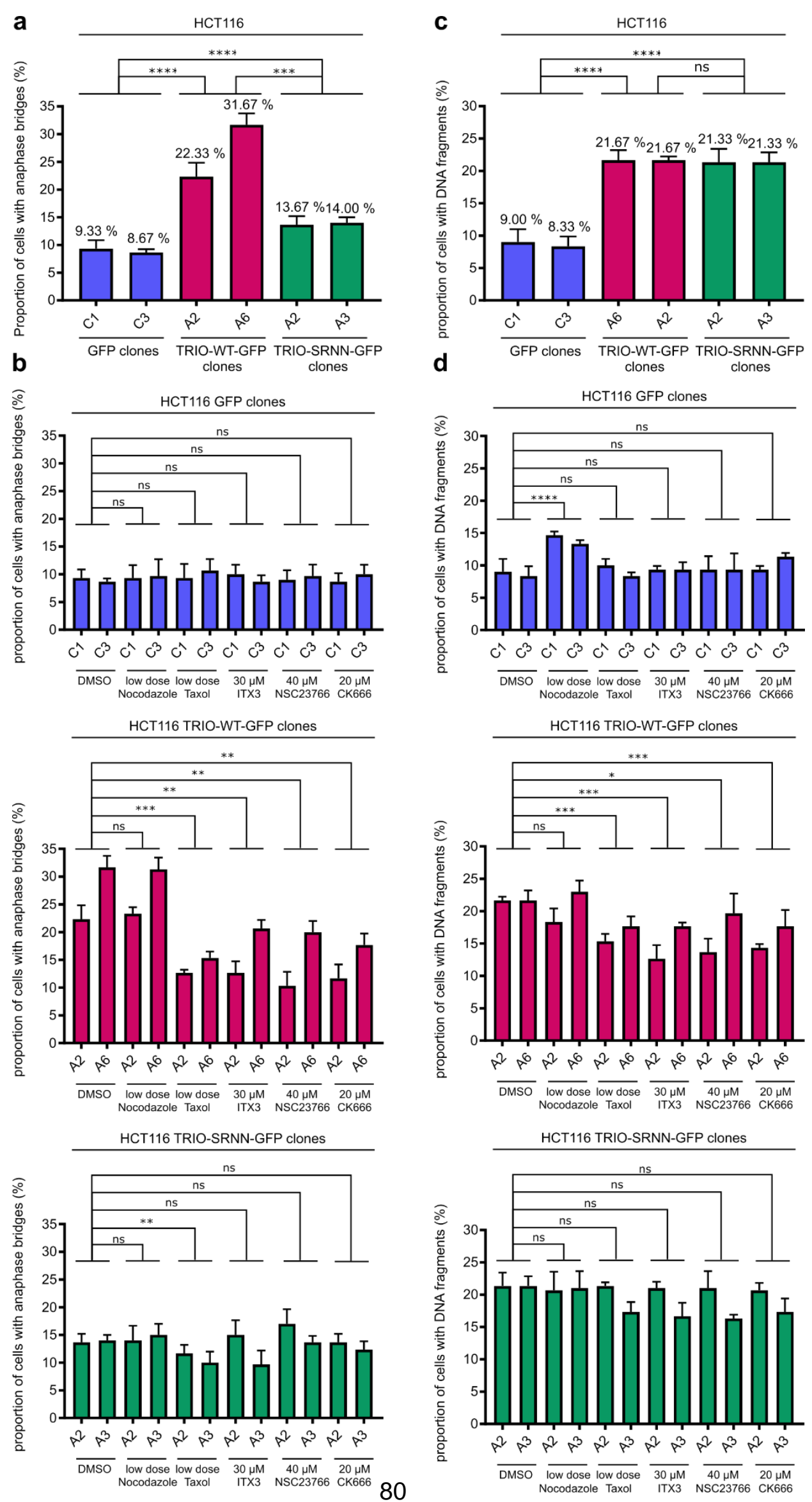
Figure 3.12: TRIO overexpression causes an increase in anaphase bridges and DNA fragments that is only partially rescuable by low dose Taxol or inhibition of TRIO GEF-D1, Rac1 or Arp2/3. (ab) Anaphase bridge formation is increased in HCT116 TRIO-WT-GFP clones in a strong, but partially rescuable manner, whereas the increase is slight and not rescuable for TRIO-SRNN-GFP clones. Bar graphs show the amount of cells from Figure 3.11a-b with anaphase bridges from three independent experiments $(n=100$ analyzed anaphase cells per experiment, mean $\pm S E M$, $t$-test). (c-d) The occurrence of DNA fragments is similarly increased in both HCT116 TRIO-WT-GFP and TRIO-SRNN-GFP clones, with being rescuable in part in TRIO-WT-GFP clones. DNA fragments were defined as Hoechst positive fragments located separately from the two-pole oriented chromosome masses of anaphase cells without CENP-C signal. Bar graphs show the proportion of cells from Figure 3.11a-b with anaphase DNA fragments from three independent experiments $(n=100$ analyzed anaphase cells per experiment, mean \pm SEM, $t$-test).

TRIO GEF-D1 activity that is involved in the generation of the observed structural abnormalities. Further research would need to be done to figure out whether the other TRIO target is GEF-D2 activated RhoA or whether it is another TRIO interaction partner independent of its GEF function.

In order to finally prove the effect of the abovementioned treatments on karyotype variability, single cell subclones were isolated from HCT116 cell clones expressing GFP, TRIO-WT-GFP or TRIOSRNN-GFP and treated with low dose Taxol, low dose Nocodazole, the TRIO inhibitor ITX3, the Rac1 inhibitor NSC23766 or the Arp2/3 inhibitor CK666 for further 30 generations. Counting of metaphase chromosome spreads affirmed the results illustrated in e, as they also showed a strong increase in cells with a varying chromosome number only for wildtype TRIO expressing subclones (Figure 3.13a). Treatments with the abovementioned drugs did not affect the karyotype stability of GFP or TRIO-SRNN-GFP expressing subclones, except from low dose Nocodazole treatment that led to an increase of cells with a chromosome number deviating from modal to up to $26.7 \%$ (Figure $3.13 \mathrm{~b}$ ). This chromosomal instability inducing effect of subnanomolar concentrations of Nocodazole has also been described before from our group (Ertych et al., 2014). Being in line with the abovementioned lagging chromosome counts, there was no further increase in karyotype variability in Nocodazole treated, but a rescue in Taxol treated wildtype TRIO expressing subclones (Figure 3.13b). This supports the suggestion, that cells with proper microtubule dynamics but TRIO overexpression already exhibit an increased amount of EB1bound TRIO when compared to control cells, thereby also being prone to Taxol-induced disruption of the EB1-TRIO interaction. When looking at the result of the other treatments in TRIO- WT-GFP expressing clones, inhibition of Rac1 and Arp2/3 showed a strong rescue of karyotype variability, while inhibition of TRIO GEF-D1 by ITX3 caused a mild rescue (Figure 3.13b). Taken as a whole, this again supports the pathway introduced at the beginning of the study being composed of microtubule plus-tip bound EB1 that interacts with TRIO, thereby leading to local 
Results

a

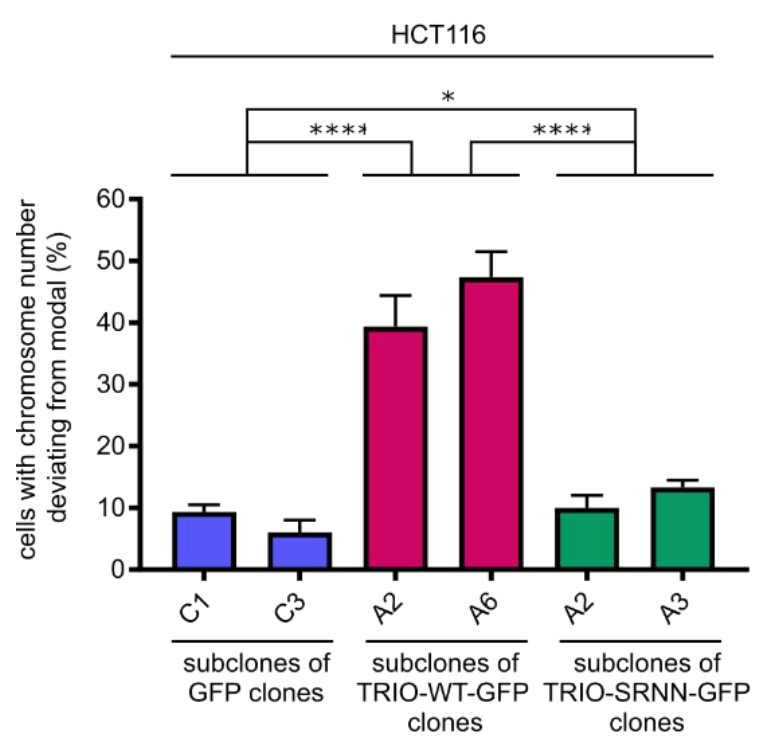

C

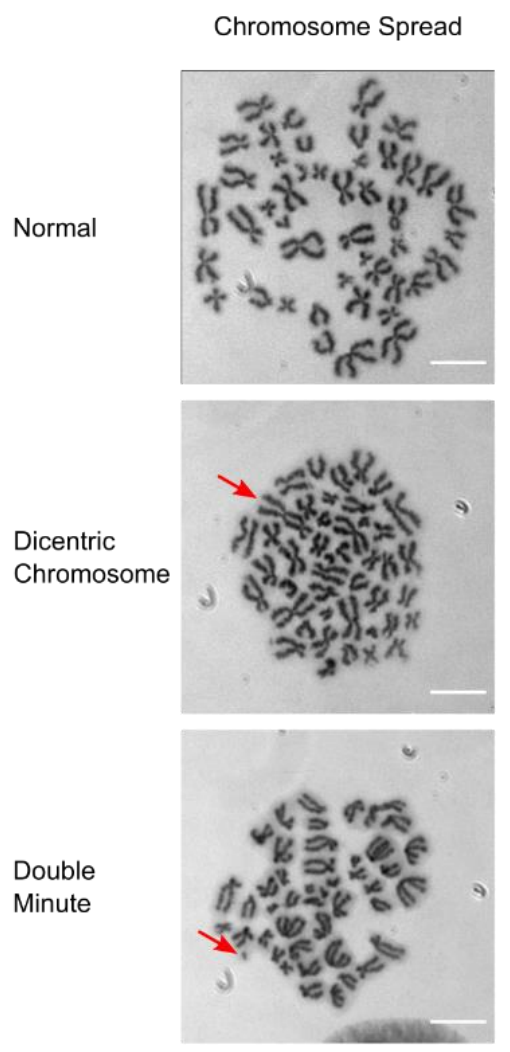

b
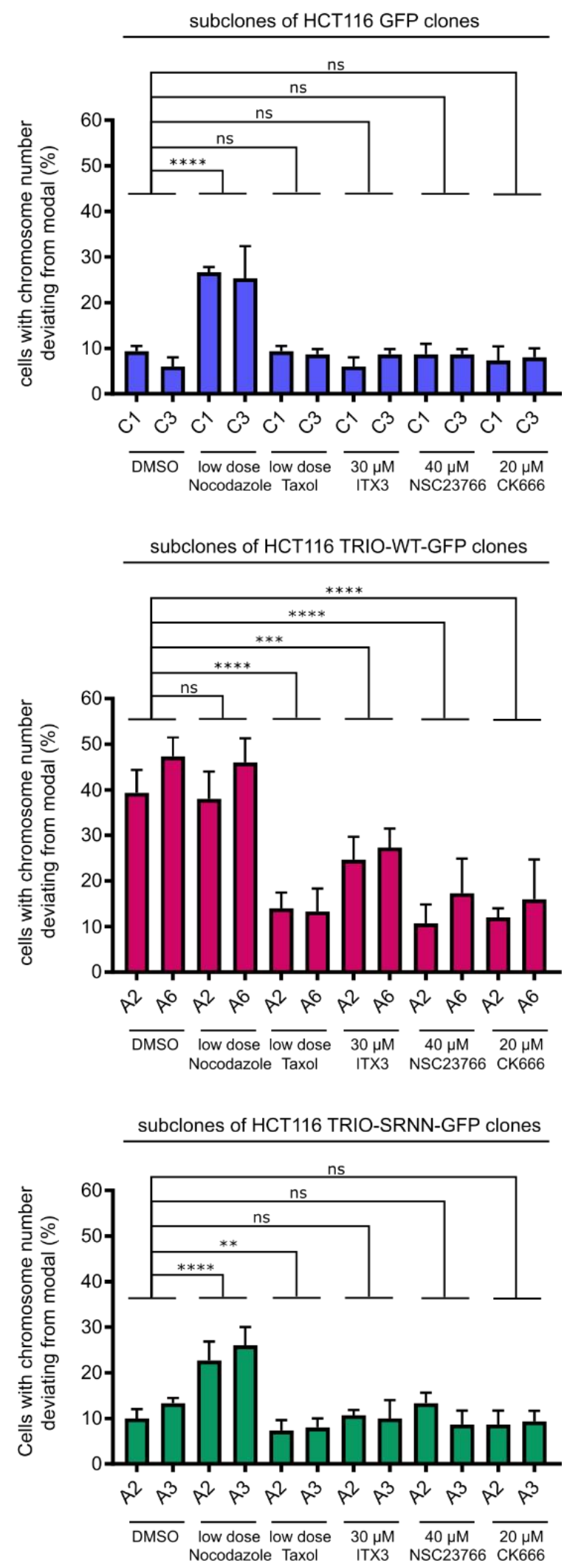
Figure 3.13: TRIO overexpression induced karyotype variability in HCT116 cells is rescuable by low dose Taxol or by inhibition of TRIO GEF-D1, Rac1 or Arp2/3. (a) HCT116 cell clones expressing wildtype TRIO, but not EB1 binding deficient TRIO exhibit an increase in karyotype variability. HCT116 cell clones expressing GFP, TRIO-WT-GFP or TRIO-SRNN-GFP were generated as previously described and used for further isolation of single cell subclones that have been cultivated for about 30 generations (DMSO control in b). Metaphase chromosome spreads were prepared for karyotype analysis. Bar graphs show the proportion of cells with a chromosome number deviating from the modal number $(n=50$ metaphase spreads for three subclones per originated clone were counted, mean \pm SEM, $t$-test). (b) Karyotype variability induced by wildtype TRIO overexpression is rescuable by low dose Taxol as well as inhibition of TRIO GEFD1, Rac1 or Arp2/3. Abovementioned HCT116 cell clones were used for further isolation of single cell subclones that have been cultivated for approximately 30 generations in the presence of DMSO (control), $0.5 \mathrm{nM}$ Nocodazole, $0.5 \mathrm{nM}$ Taxol, $30 \mu \mathrm{M}$ ITX3, $40 \mu \mathrm{M}$ NSC23766 or $20 \mu \mathrm{M}$ CK666 and were used for the preparation of metaphase chromosome spreads. Bar graphs show the proportion of cells with a chromosome number deviating from the modal number $(n=50$ metaphase spreads for three subclones per originated clone were counted, mean \pm SEM, $t$-test). (c) Representative light microscopy images of metaphase spreads from HCT116 cell clones from (a) with or without the occurrence of a dicentric chromosome or a double minute. Scale bar $10 \mu \mathrm{m}$.

Rac1 activation, which in turn activates the F-actin polymerization-promoting Arp2/3 complex. However, the reason why the rescue of karyotype variability after treatment with the TRIO GEFD1 inhibitor ITX3 was not as pronounced as after inhibition of Rac1 or Arp2/3 remained unknown for now.

Parallel to the finding of structural abnormalities during lagging chromosome counts, metaphase chromosome spreads of the analyzed subclones revealed in addition to whole chromosomal instability (W-CIN) also signs of structural chromosomal instability (S-CIN), from which many can be also analyzed via Giemsa chromosome staining (Abe et al., 2015; Lepage et al., 2019; Ryu et al., 2016). This was demonstrated by the occurrence of dicentric chromosomes and acentric chromosomes, with the latter one appearing in pairs as so-called double minutes (Bao et al., 2016) (Figure 3.13c). Dicentric chromosomes are genetically unstable structures, as when microtubules bound to the two centromeres of one sister chromatid pull to opposite poles, anaphase bridges are formed, followed by DNA breakage (Lopez et al., 2015). This process is part of so called breakage/fusion/bridge (B/F/B) cycles and is known to produce rearrangements, such as the observed double minutes that are probably visible as DNA fragments during anaphase as illustrated in Figure 3.11c (Lo et al., 2002). While the induction of dicentric chromosome and double minute formation was strong in wildtype TRIO expressing subclones, it was slight to mediate in subclones expressing EB1 binding deficient TRIO (Figure 3.14a and c). Similar to the results from lagging chromosome counts, the formation of dicentric chromosomes could only partially be rescued in TRIO-WT-GFP subclones via treatment with low dose Taxol or inhibitors 
Results
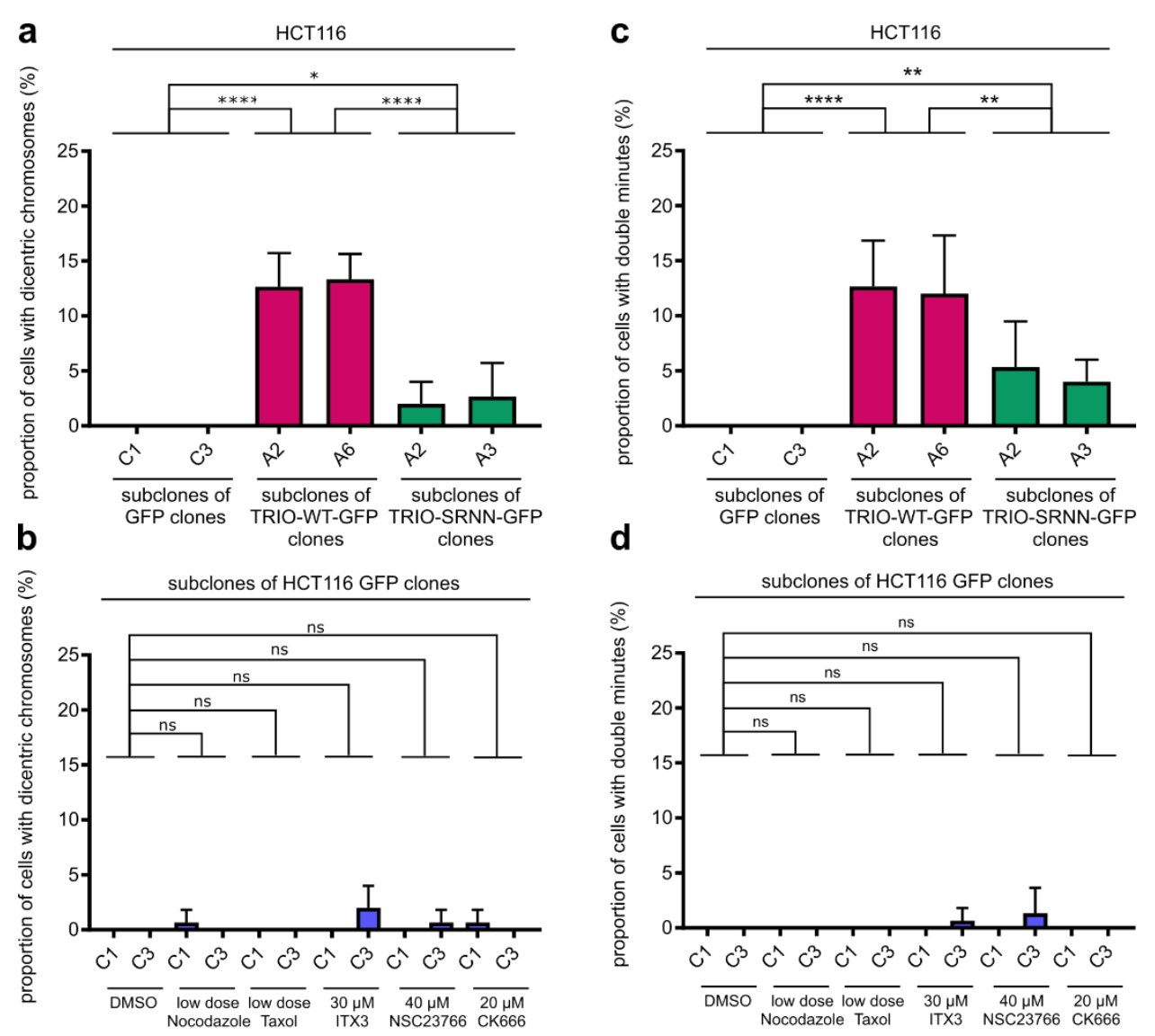

subclones of HCT116 TRIO-WT-GFP clones
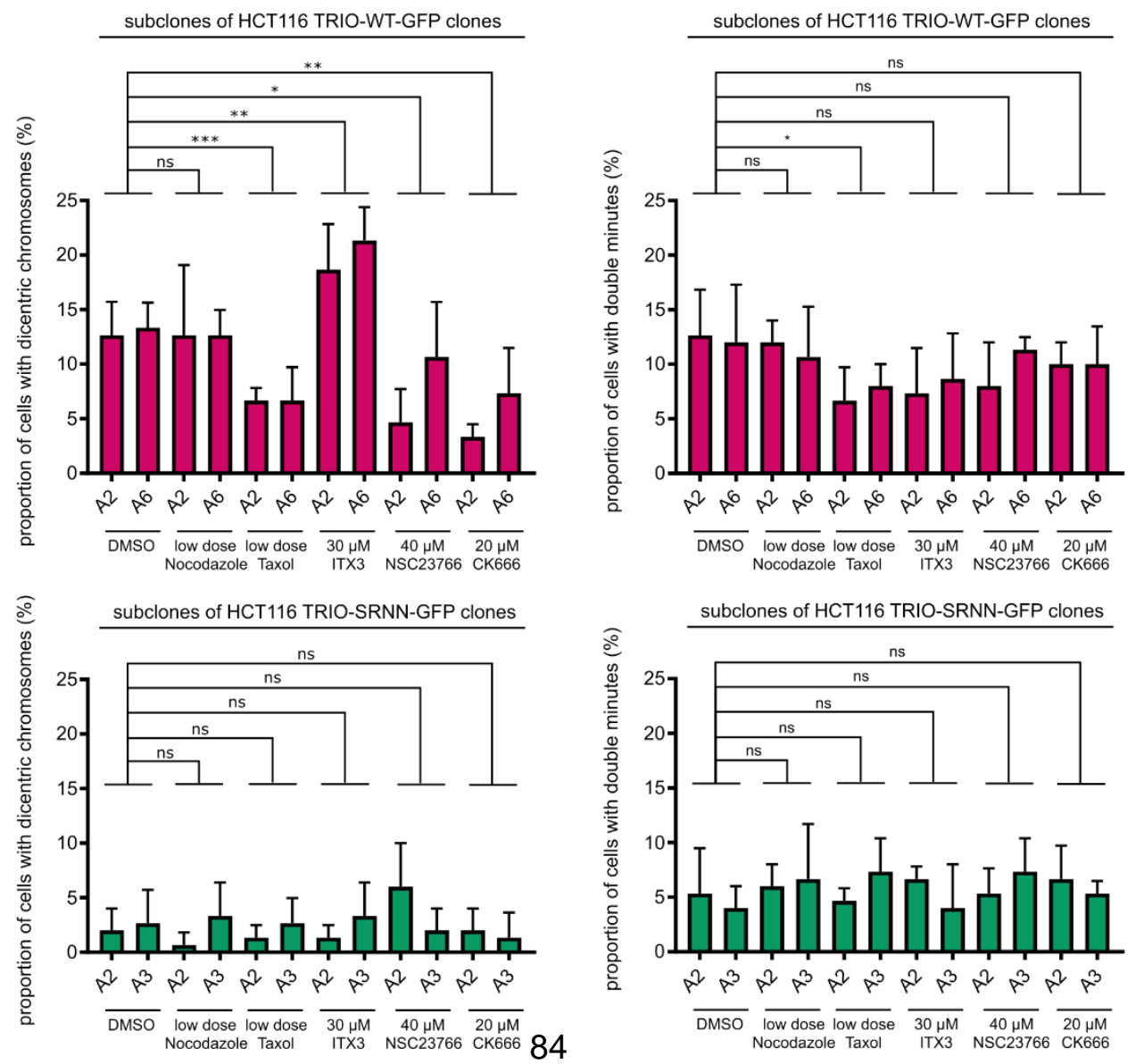
Figure 3.14: Overexpressed TRIO induces the formation of dicentric chromosomes and double minutes that are only partially or not rescuable by low dose Taxol or inhibition of Rac1 or Arp2/3. (ab) Dicentric chromosome formation is strongly induced, but partially rescuable in HCT116 TRIO-WT-GFP subclones and slightly induced in TRIO-SRNN-GFP subclones. Bar graphs show the proportion of cells from Figure 3.13a-Figure 3.11b with dicentric chromosomes $(n=50$ metaphase spreads for three subclones per originated clone were counted, mean \pm SEM, $t$-test). (c-d) The generation of double minutes is strongly induced by wildtype TRIO and slightly induced by EB1 binding deficient TRIO expression in HCT116 cell clones and cannot be rescued by the given treatments. Bar graphs show the amount of cells from Figure $3.13 a-b$ with double minutes $(n=50$ metaphase spreads for three subclones per originated clone were counted, mean \pm SEM, $t$-test).

against Rac1 or Arp2/3, but there was no further decrease detected for TRIO-SRNN-GFP subclones (Figure 3.14b). Surprisingly, treatment with the TRIO GEF-D1 inhibitor ITX3 even caused a significant increase in dicentric chromosome formation, but only in wildtype TRIO expressing subclones. This probably explains the only mild rescue of karyotype variability seen for ITX3 in TRIO-WT-GFP subclones, as a dicentric chromosome is counted as one chromosome in metaphase chromosome spreads, thereby resulting in a chromosome number that is reduced by one. Due to the finding that ITX3 shows this effect only in wildtype TRIO expressing subclones, it might require EB1 binding of TRIO. However, the actual mechanism by which ITX3 enhances the generation of dicentric chromosomes in these cells remained unknown. When looking at double minute formation, TRIO-WT-GFP subclones showed a high and TRIO-SRNN-GFP subclones a mediate inductive effect (Figure 3.14d). This induction could slightly be rescued in wildtype TRIO expressing subclones by low dose Taxol treatment and to a very mild, but statistically not significant extend by inhibition of TRIO, Rac1 or Arp2/3. Again, the mediate induction observed for EB1 binding deficient TRIO expressing subclones was not rescuable by the given treatments.

Taken together, these results support the suggestion established on the base of anaphase bridge and DNA fragment counts, that proposes the existence of a S-CIN-inducing pathway independent of the binding of TRIO to EB1 and its GEF-D1 activity. In this context, further research would be needed to unravel the function and targets of TRIO as well as other involved components. 


\subsection{Increased mitotic Rac1 activity induces spindle axis misalignment, chromosome mis- segregation and chromosomal instability}

The results described above already indicate the existence of a mitotic microtubule plus-tip, EB1, TRIO, Rac1, Arp2/3 dependent pathway for the induction of chromosomal instability. However, finding out Rac1 as target of TRIO within this pathway has only been shown by inhibition of TRIO GEF-D1 and Rac1. In order to further confirm Rac1 as component of the pathway, the effect of an increased activation of the given pathway downstream of TRIO on chromosomal instability was investigated by analyzing mitotic cells with a high Rac1 activity. So far, Rac1 activity was described to be downregulated upon entry into mitosis and only present at cell poles, but excluded at the cleavage furrow during cytokinesis (Yoshizaki et al., 2003). The expression of constitutively active Rac1 was shown to inhibit cytokinesis and thereby induced the generation of multinucleated cells (Yoshizaki et al., 2004). However, the effect of a high Rac1 activity during earlier mitotic phases has not been investigated, yet. In order to do so, wildtype MCF10A cells and genome-edited MCF10A cells, in which one RAC1 allele encodes the constitutively active Q61L mutation, were examined for possible differences in prometaphase spindle axis alignment and chromosome segregation defects in anaphase. Microtubule plus-end assembly rates were determined beforehand and revealed no difference between MCF10A cells expressing wildtype or constitutively active Rac1 (Rac1-CA), thereby excluding a possible impact of constitutively active Rac1 on microtubule dynamics acting upstream in the introduced pathway (Figure 3.15a). In order to investigate whether a high mitotic Rac1 activity, that could arise by the suggested microtubule plus-tip, EB1, TRIO dependent pathway, had an actual impact on spindle positioning, the spindle axis angle was determined in MCF10A cells with or without expressing constitutively active Rac1. In fact, there was a slight, but significant increase in the spindle axis angle of MCF10A cells expressing constitutively active Rac1 $\left(13.88^{\circ}\right)$ when compared to wildtype cells $\left(8.25^{\circ}\right.$; Figure 3.15b). However, this increase was not as pronounced as known for chromosomally unstable cells, that usually showed a spindle axis angle of about $20^{\circ}$ or higher (Ertych et al., 2014; Berger, 2016; Schermuly, 2019). This smaller increase determined for MCF10A Rac1-CA cells might be explained by the additionally observed greater diameter and, thus, centrosome to centrosome distance of these cells in prometaphase when compared to the wildtype cells (Figure 3.15c). Due to taking the distance between centrosomes into account for calculating the spindle axis angle, a large centrosome to centrosome distance resulted in a smaller spindle axis angle (Figure 3.15e and f). Most cell lines analyzed for spindle axis alignment in our group showed a centrosome to 

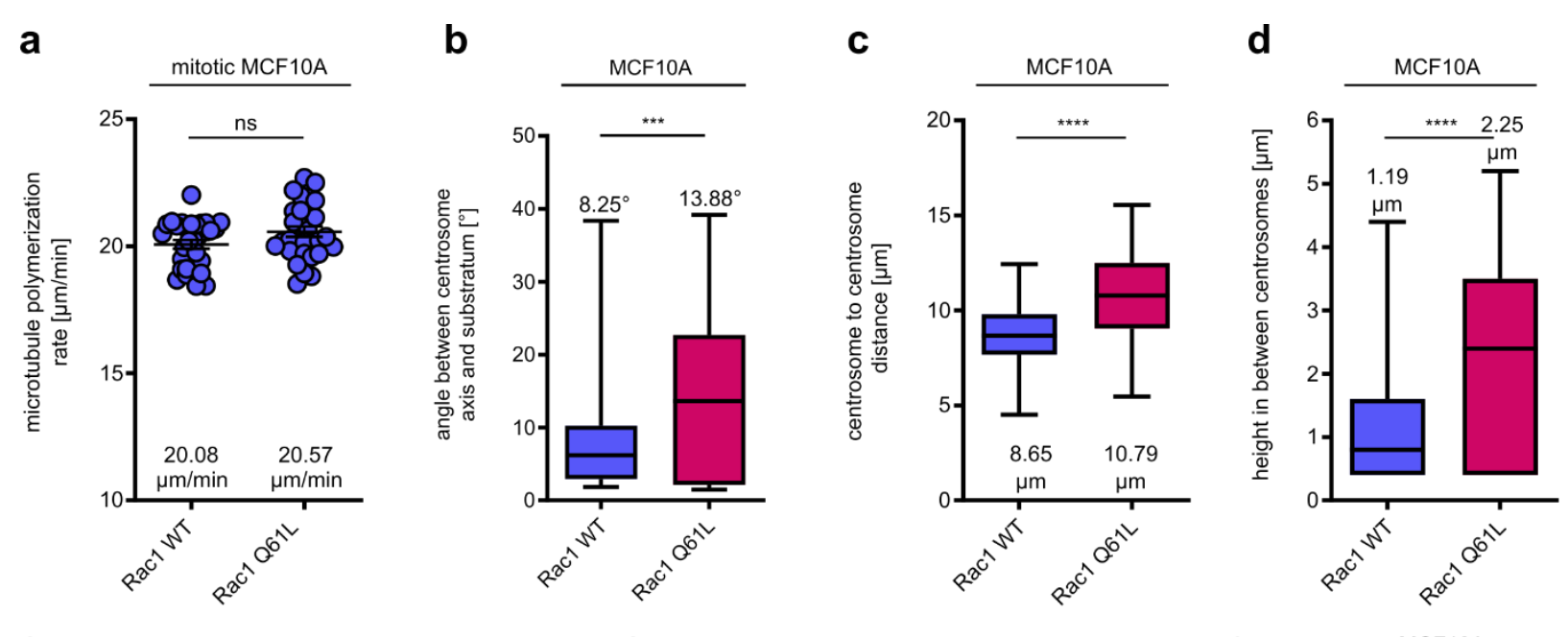

e
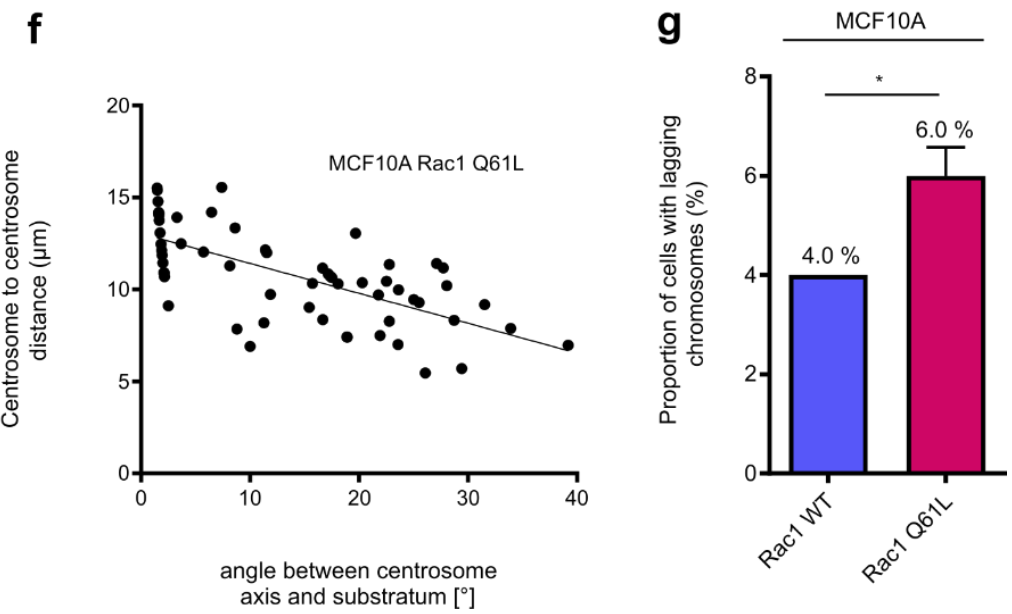

Figure 3.15: Expression of constitutively active Rac1 leads to a slight increase in spindle axis misalignment and chromosome mis-segregation. (a) Mitotic microtubule plus-end assembly rates are not affected by the expression of constitutively active Rac1. MCF10A wildtype and Rac1-CA cells were transfected with an EB3-GFP expressing plasmid and treated with $2 \mu \mathrm{M}$ DME $2 \mathrm{~h}$ before measurements. Scatter dot plots show average microtubule growth rates from 30 cells from three independent experiments (mean \pm SEM, $t$-test). (b) Spindle axis alignment is slightly increased in MCF10A Rac1-CA cells. MCF10A wildtype and Rac1-CA cells were seeded onto fibronectin-coated glass coverslips and stained for a-tubulin (microtubules), $y$-tubulin (centrosomes) and Hoechst33342 (DNA). The box and whisker plots show median, quartile and range of three independent spindle axis angle measurements $(n=20$ cells per experiment, $t$ test). Further analyzed data from (b) show that MCF10A Rac1-CA cells exhibit a greater (c) centrosome to centrosome distance and (d) height in between centrosomes than wildtype cells and the (e-f) correlation of the distance between centrosomes and the spindle axis angle. (g) The amount of lagging chromosomes is increased in MCF10A cells with constitutively active Rac1 expression. MCF10A wildtype and Rac1-CA cells were synchronized by a double thymidine block and fixed $11 \mathrm{~h}$ after release to obtain an increased proportion of anaphase cells. Cells were stained for a-tubulin (microtubules), CENP-C (centromeres) and Hoechst33342 (DNA). Chromosomes positive for Hoechst and CENP-C and located clearly separated from two pole-oriented chromosome masses in anaphase cells were counted as lagging chromosomes. Bar graphs show the proportion of cells with lagging chromosomes from three independent experiments $(\mathrm{n}=$ 100 analyzed anaphase cells per experiment, mean \pm SEM, $t$-test). 
centrosome distance of about 6-7 $\mu \mathrm{m}$ (Schermuly, 2019). When taking such a centrosome to centrosome distance into account but using the height in between centrosomes observed for MCF10A wildtype and Rac1-CA cells for calculation (Figure 3.15d), MCF10A wildtype would exhibit a spindle axis angle of about $10^{\circ}$, whereas MCF10A Rac1-CA cells had an angle of about $20^{\circ}$. This would reflect the difference between chromosomally stable and unstable cells reported

a

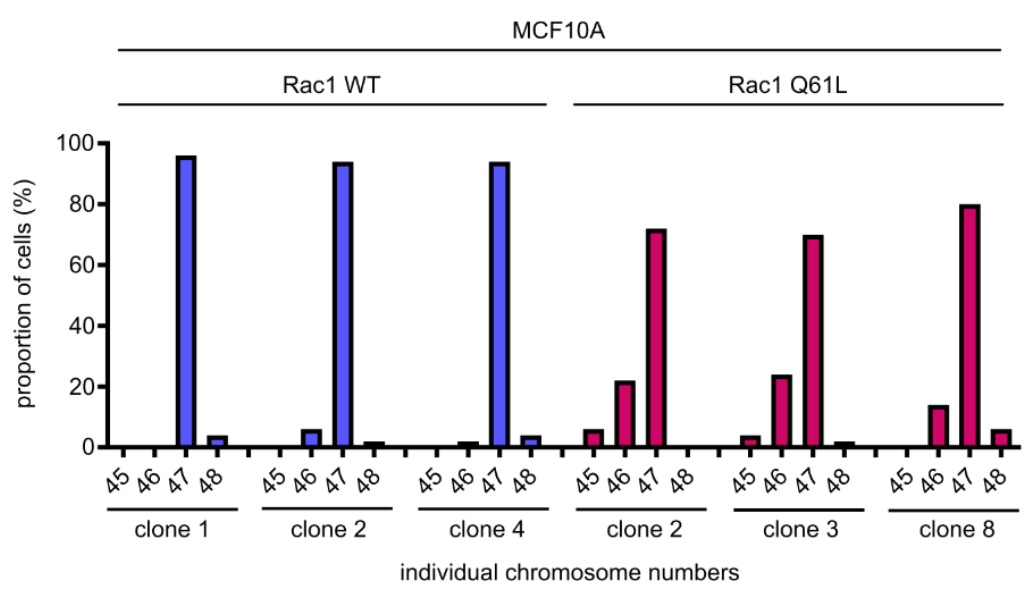

b

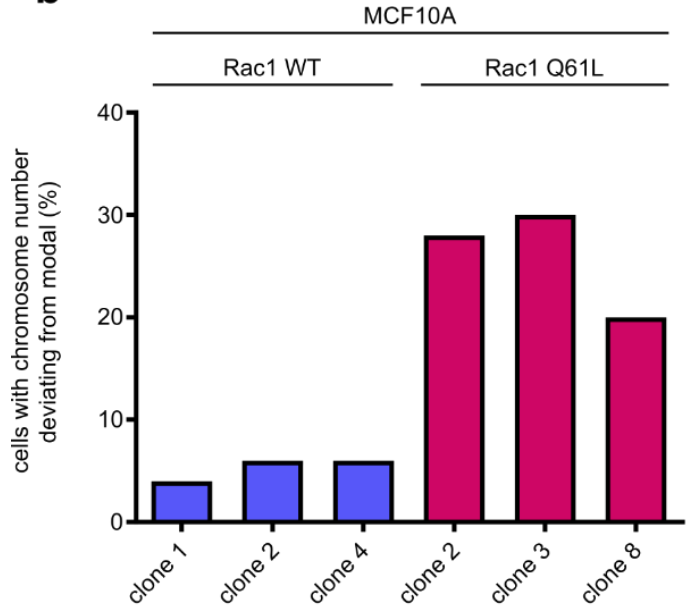

C

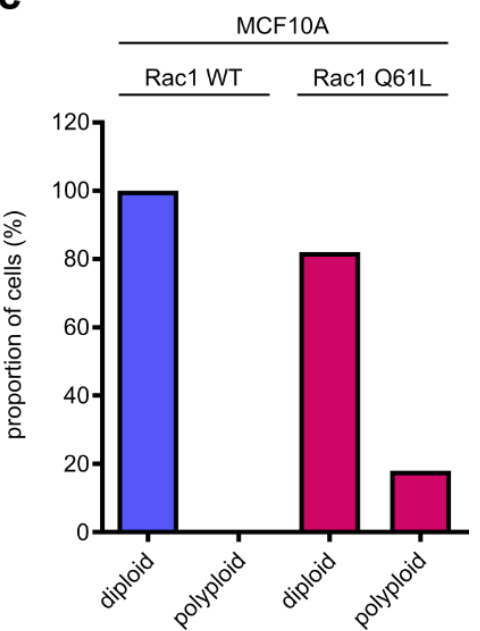

Figure 3.16: Expression of constitutively active Rac1 not only induces the generation of multinucleated cells, but also chromosomal instability. (a-b) Karyotype analysis of MCF10A cell clones demonstrating chromosomal instability for constitutively active Rac1 expressing cells. Single cell clones of MCF10A wildtype and Rac1-CA cells were isolated and cultivated for about 30 generations. Chromosomes of metaphase spreads were counted. Bar graphs in (a) show the proportion of cells with certain chromosome numbers between 45 and 48 and in (b) illustrate the proportion of cells with a chromosome number that is deviating from the modal number of $47(n=50$ metaphase spreads for each clone). (c) Expression of constitutively active Rac1 induces polyploidy. Metaphase chromosome spreads of MCF10A wildtype and Rac1-CA cells were prepared and polyploid cell spreads were counted ( $n=300$ metaphase spreads). 
previously in our group (Schermuly, 2019). Consequently, the used calculation for the spindle axis angle might usually work out but is limited to cells with about the same centrosome to centrosome distance within a specific range. In order to prove whether the increased spindle axis misalignment in MCF10A Rac1-CA cells has an impact on chromosome segregation, the amount of cells with lagging chromosomes was determined. Again, the difference between MCF10A wildtype and Rac1-CA cells was not as pronounced, but significant. While MCF10A wildtype cells only exhibited $4 \%$ lagging chromosomes, this proportion was increased to $6 \%$ for MCF10A Rac1-CA cells (Figure 3.15g). As final proof, chromosomes of metaphase spreads were counted for karyotype analysis. Therefore, single cell clones were isolated from MCF10A wildtype and Rac1-CA cells and cultivated for about 30 generations. In fact, MCF10A wildtype cell clones showed a stable karyotype with only 4-6\% cells having a chromosome number deviating from the modal number of 47 , whereas this was $20-30 \%$ for the MCF10A Rac1-CA cell clones (Figure 3.16a and b). Additionally, the occurrence of polyploidy was observed, but only for MCF10A Rac1-CA cells (Figure 3.16c). Polyploid cells were not taken into account for the abovementioned chromosome counting of metaphase spreads. The finding of polyploidy in MCF10A Rac1-CA cells confirms the previously described generation of multinucleated cells due to inhibition of cytokinesis by expression of constitutively active Rac1 (Yoshizaki et al., 2004).

Summed up, a high mitotic Rac1 activity was shown to act downstream of microtubule dynamics, where it induces increased spindle axis misalignment, chromosome mis-segregation and chromosome instability. In addition, these results further confirmed Rac1 as the sought-after TRIO target that links microtubule plus-tip bound EB1 with the induction of chromosome instability.

\subsection{The mitotic F-actin cytoskeleton is regulated by a pathway containing microtubule plus- tip bound EB1, TRIO, Rac1 and Arp2/3}

So far, this study has contributed important evidence for the introduced pathway and has revealed missing links within this pathway. But there was still a missing link between the by now most downstream component Arp2/3 and spindle axis misalignment. In this context, Kwon et al. (2015) found out that a mitotic Arp2/3 dependent actin cloud structure is involved in spindle positioning by forwarding pulling forces generated from retraction fibers to centrosomes via binding to astral microtubules. Whether these subcortical actin clouds were also regulated by the analyzed pathway should be figured out by looking for changes in the cloud structure after inhibition of pathway components. Therefore, chromosomally unstable SW620 and HT29 cells were 
transfected with plasmids expressing UtrophinCH-GFP for the detection of F-actin and H2B-RFP for DNA visualization. During live cell imaging, metaphase cells were chosen for the analysis of actin clouds, as described elsewhere (Kwon et al., 2015; Mitsushima et al., 2010). Differences in the actin cytoskeleton were determined by calculating the fluorescence intensity of the F-actin signal within a metaphase cell. Therefore, the pixel intensity derived from a generated line scan of a metaphase cell was used (Figure 3.17a). To differentiate between the signal intensity of the

a

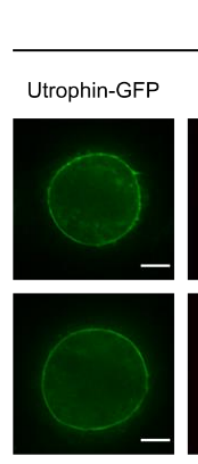

SW620
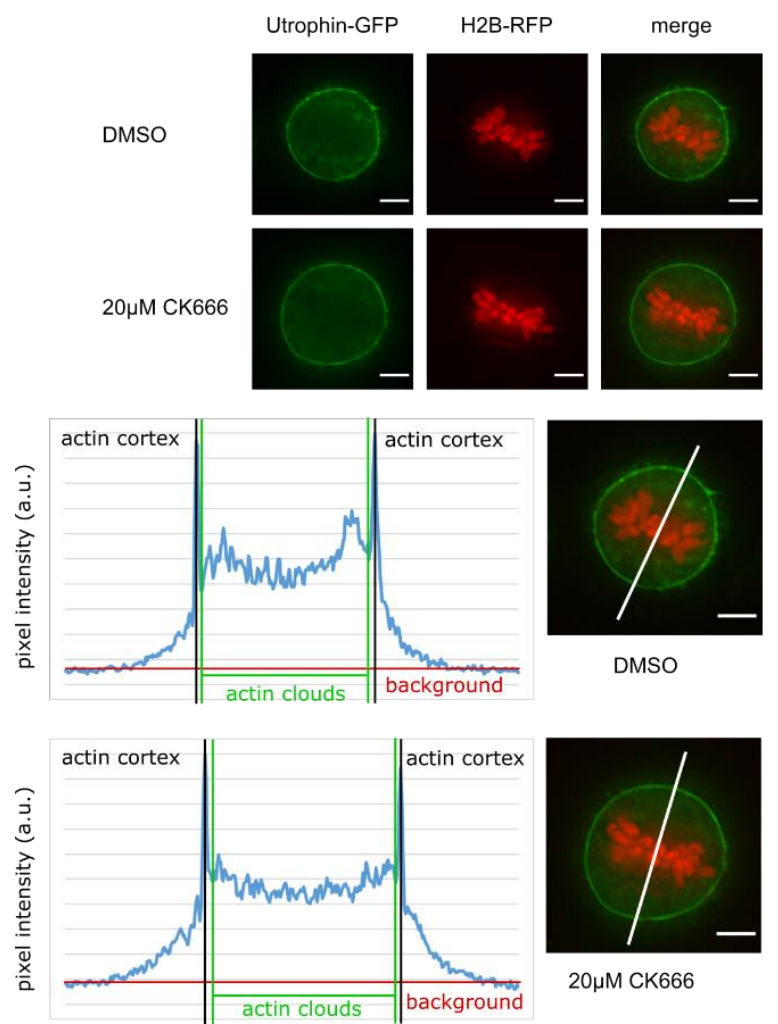

b
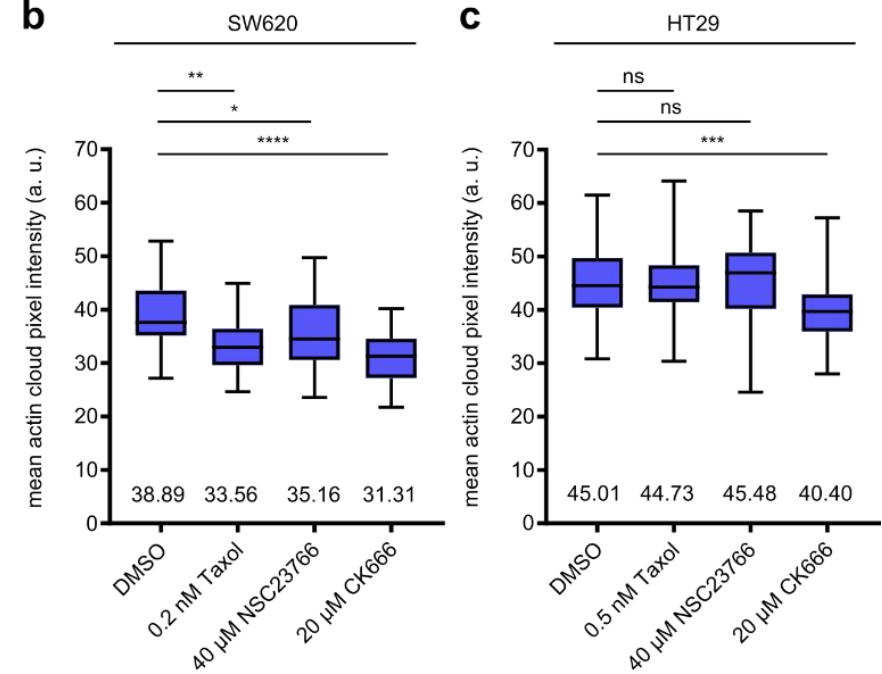

Figure 3.17: Mitotic actin cloud structures are regulated by a fraction of Arp2/3 that does not seem to be involved in the EB1, TRIO, Rac1, Arp2/3 dependent pathway. (a) Mitotic SW620 cells exhibit subcortical actin clouds that are decreased after Arp2/3 inhibitor treatment. SW620 cells were transfected with plasmids encoding UtrophinCH-GFP (F-actin, green) and H2B-RFP (DNA, red) and treated with DMSO (control) or $20 \mu \mathrm{M}$ of the Arp2/3 inhibitor CK666 for $16 \mathrm{~h}$. Cell were analyzed by live cell microscopy and line scans of pixel intensity values from metaphase cells were used to calculate the actin cloud and actin cortex signal intensity. Scale bar $5 \mu \mathrm{m}$. Actin cloud signal intensities in (b) SW620 and (c) HT29 cells are decreased after Arp2/3 inhibition, but they show only a slight (SW620) or no (HT29) reduction after low dose Taxol or Rac1 inhibitor treatment. SW620 and HT29 cells were transfected as described in (a) and treated with DMSO, 0.2-0.5 nM Taxol, $40 \mu \mathrm{M}$ NSC23766 or $20 \mu \mathrm{M}$ CK666 $16 \mathrm{~h}$ before live cell microscopy. Box and whisker plots showing the median, quartile and range of the mean actin cloud signal intensities (normalized to the corresponding actin cortex signal intensity) calculated from line scans of three independent experiments $(n=10-20$ cells per experiment, $t$-test). 
actin cortex and the clouds, pixel intensities representing these regions were used for the calculation. As inhibition of Arp2/3 was described to only inhibit the formation of the mitotic actin cloud structure, but not the actin cortex (Mitsushima et al., 2010), and in order to take the effect of different expression levels into account, the actin cortex intensity was used to normalize actin cloud intensity values. This was done under the assumption that the level of UtrophinCH-GFP expression did not affect its distribution in the actin structures of the cell, meaning the ratio between UtophinCH-GFP in the actin cortex and the actin clouds would stay the same in cells with different expression levels. When comparing the actin cloud signal intensity of metaphase SW620 cells with or without treatment with the Arp2/3 inhibitor CK666, there was a clear reduction detectable for inhibitor treated cells, that has also been shown by Mitsushima et al. (2010) (Figure 3.17a-b). This decrease in actin cloud signal intensity was also shown for Arp2/3 inhibitor treated HT29 cells (Figure 3.17c). However, when interfering with the introduced pathway by low dose Taxol-induced disruption of EB1 and TRIO or by inhibition of Rac1 using the inhibitor NSC23766, there was only a slight reduction of actin cloud signal intensity detected for SW620 cells (Figure 3.17b) and no reduction for HT29 cells (Figure 3.17c). These results suggest that the fraction of Arp2/3 involved in actin cloud regulation is not constituting the fraction of Arp2/3 that is part of the EB1, TRIO, Rac1 dependent pathway. At this point it should be noted that the performed procedure for the determination of actin cloud intensity was done under the assumption that the given treatments did not affect the actin cortex but only the actin clouds, as described for CK666mediated Arp2/3 inhibition by Mitsushima et al. (2010). However, Bovellan et al. (2014) showed that Arp2/3 was also important for the mitotic actin cortex (Bovellan et al., 2014), which in turn was reported to be involved in spindle positioning (Kunda \& Baum, 2009). In order to prove an involvement of the actin cortex in the introduced pathway, actin cortex signal intensities were determined in addition to actin cloud intensities using fixed cells stained with fluorescently-labeled phalloidin for F-actin visualization. This procedure allowed for a uniform F-actin staining, thereby avoiding the need for normalization as given in case of different UtrophinCH-GFP expression levels. In order to figure out, whether the actin cytoskeleton structure in chromosomally stable and unstable cells is affected differently after interfering with the introduced pathway, HCT116 (chromosomally stable) as well as SW620 and SW480 (chromosomally unstable) cells were analyzed. To manipulate the pathway at different parts, the cells were treated with low dose Taxol, the TRIO inhibitor ITX3, the Rac1 inhibitor NSC23766 or the Arp2/3 inhibitor CK666. Interestingly, chromosomally unstable SW620 and SW480 cells showed a clear reduction of the 
Results

a

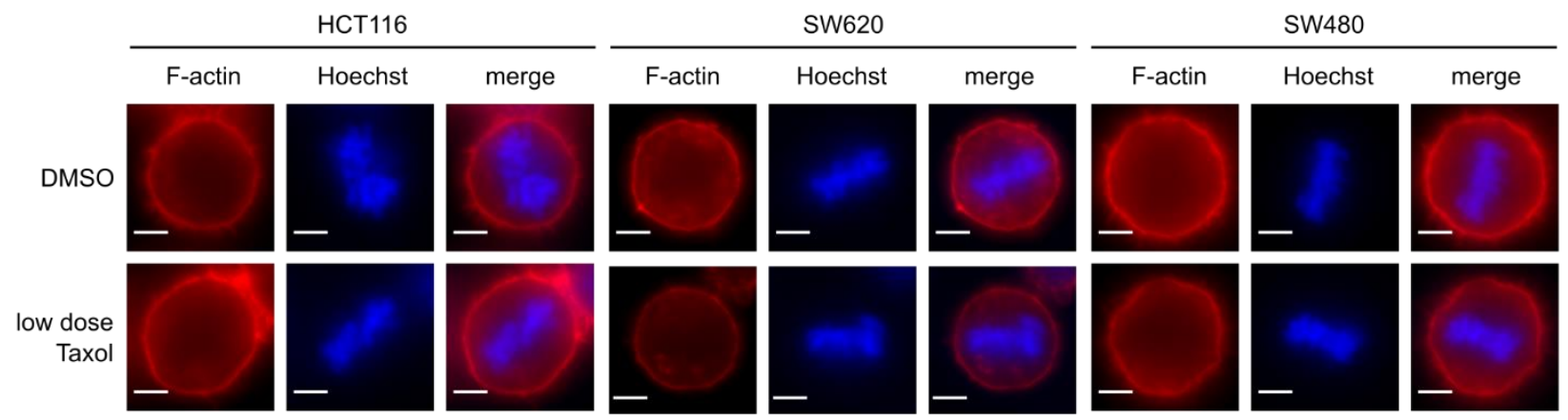

b

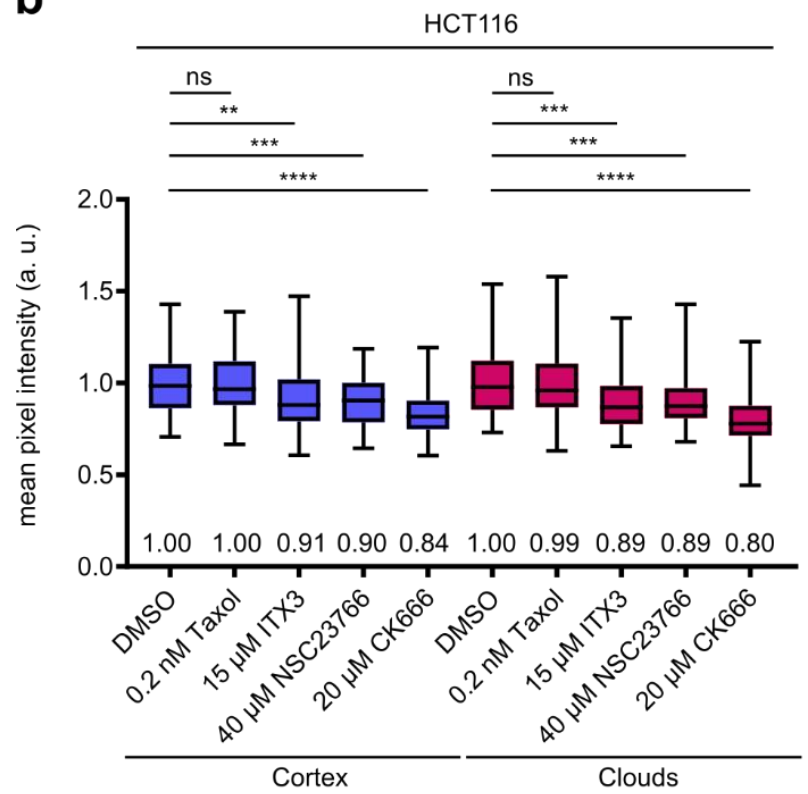

d

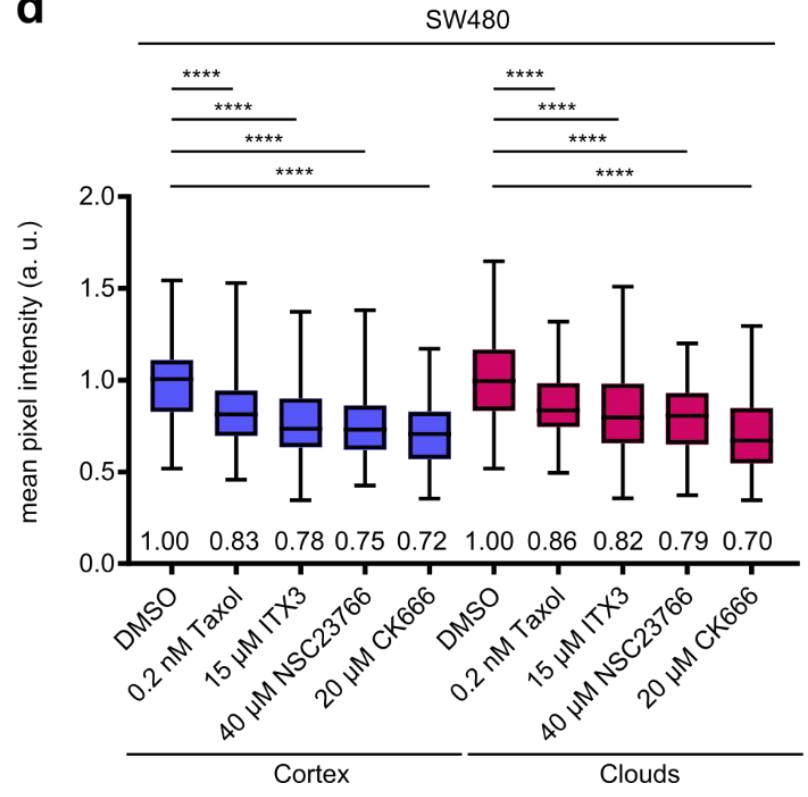

C SW620

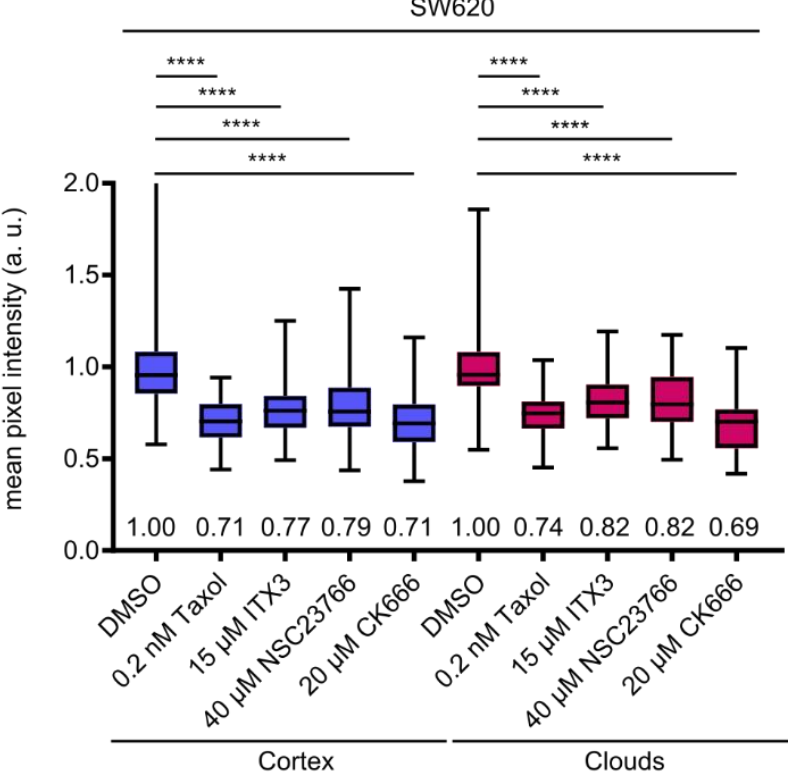


Figure 3.18: The mitotic F-actin cytoskeleton is regulated by a microtubule dynamic dependent pathway in chromosomally unstable cells. (a) Representative immunofluorescence images of the F-actin cytoskeleton of HCT116 (non-CIN), SW620 (CIN) and SW480 (CIN) cells with or without low dose Taxol treatment showing a Taxol-induced decrease in the actin signal intensity only in CIN cells. Cells were treated with DMSO (control) or $0.2-0.5 \mathrm{nM}$ Taxol for $16 \mathrm{~h}$ and stained for F-actin (AlexaFluor ${ }^{\mathrm{TM}} 594$ Phalloidin; red) and DNA (Hoechst33342; blue). Scale bar $5 \mu \mathrm{m}$. Quantification of actin cortex and cloud signal intensities showing a decrease in (b) HCT116, (c) SW620 and (d) SW480 cells after inhibition of TRIO, Rac1 or Arp2/3, but only a decrease for SW620 and SW480 cells after low dose Taxol treatment. Cells were treated with DMSO, 0.2-0.5 nM Taxol, $15 \mu \mathrm{M}$ ITX3, $40 \mu \mathrm{M}$ NSC23766 or $20 \mu \mathrm{M}$ CK666 for $16 \mathrm{~h}$ and stained as described in (a). Box and whisker plots showing the median, quartile and range of the mean actin cortex and cloud intensity calculated from metaphase line scans of three independent experiments $(n=20-36$ cells per experiment, $t$-test).

actin cortex as well as the actin cloud signal intensity after low dose Taxol treatment while chromosomally stable HCT116 cells stayed unaffected (Figure 3.18a-d). Consequently, an increased microtubule plus-end assembly rate in chromosomally unstable cells seems to induce increased cytoskeletal F-actin polymerization that is rescuable by restoring proper microtubule plus-end assembly rates using low dose Taxol. In contrast, the actin cytoskeleton of chromosomally stable cells is not affected as they exhibit normal microtubule plus-end assembly rates that do hardly decrease after treatment with low dose Taxol (Ertych et al., 2014). These results indicate that microtubule dynamics are involved in the regulation of mitotic actin cytoskeleton structures, that in turn play an important role for spindle axis alignment. Having a look at the effect of the other inhibitor treatments, it was striking that both chromosomally unstable (SW620 and SW480) and chromosomally stable (HCT116) cells showed a clear decrease in actin cortex and cloud signal intensities (Figure 3.18c-d). However, it is of note that the decrease was more pronounced in SW620 (up to 29\% decrease in cortex and 31\% in cloud signal intensity) and SW480 (up to $28 \%$ decrease in cortex and $30 \%$ in cloud signal intensity) than in HCT116 cells (up to $16 \%$ decrease in cortex signal and $20 \%$ in cloud signal intensity). This again suggests that the introduced pathway might be stronger activated in chromosomally unstable cells as an inhibition of pathway components has a stronger impact on the actin cytoskeleton downstream of the pathway. Besides, it is not surprising that the actin cytoskeleton of chromosomally stable HCT116 cells is also affected by inhibition of the overall pool of TRIO, Rac1 or Arp2/3, as these are important actin cytoskeleton regulators in general.

In order to exclude possible off-target effects of the used drugs, siRNA-mediated knock downs were performed, followed by actin cytoskeleton signal intensity measurements. First, proteins involved in upstream parts of the pathway were knocked down in SW620 and SW480 cells. Therefore, cells were transfected with siRNA for CKAP5, coding for the microtubule polymerase 
ch-TOG whose knockdown was shown to cause a decrease in microtubule plus-end assembly rates (Ertych et al., 2014), with siRNA for EB1 or for TRIO. The knock down efficiency was verified by Western blots (Figure 3.19a). Both SW620 and SW480 cells showed a clear reduction in actin cortex and cloud signal intensities after knockdown of CKAP5, EB1 or TRIO, thereby confirming the results from inhibitor treated cells with an additional proof of the involvement of EB1 (Figure 3.19b-c).

a

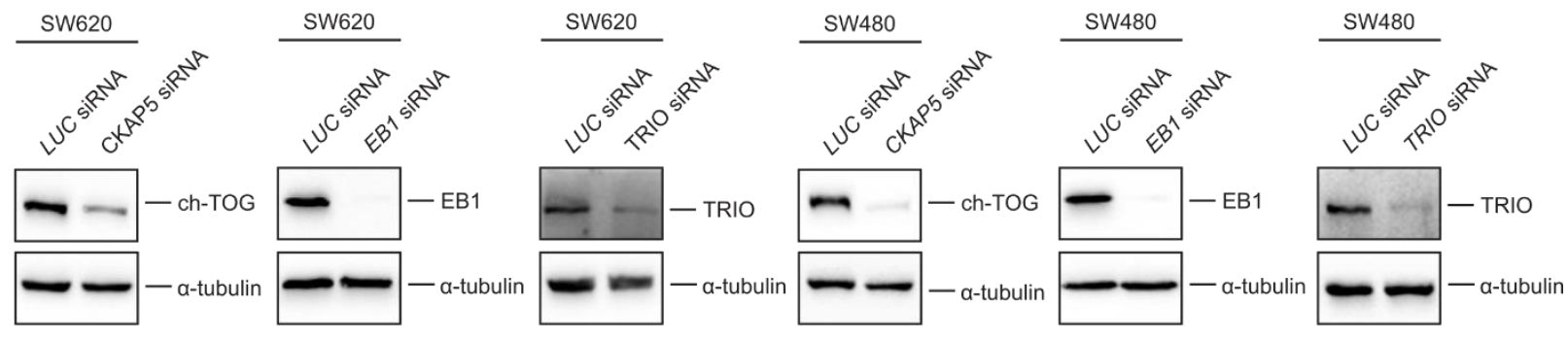

b

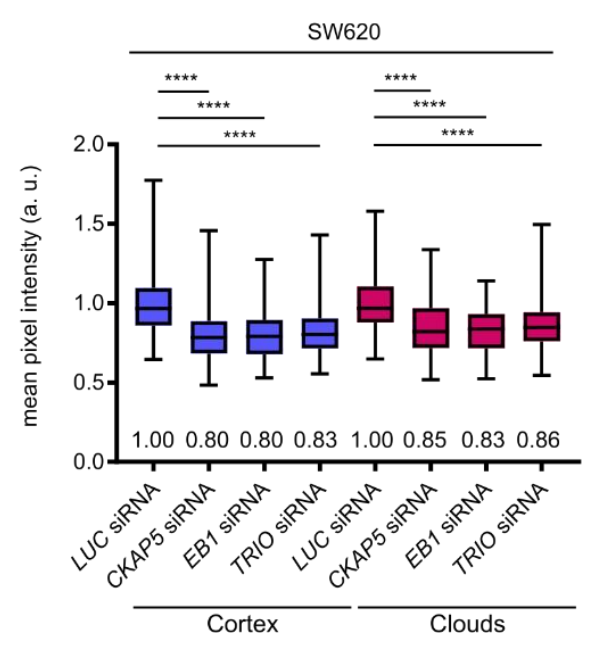

C

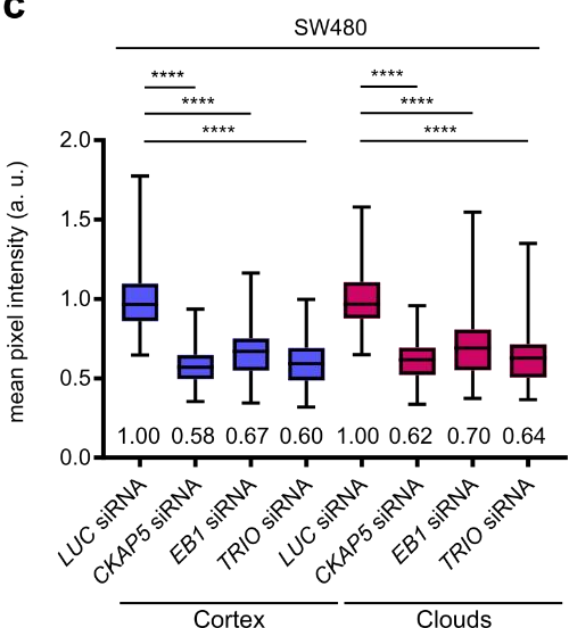

Figure 3.19: Knock down of CKAP5, EB1 or TRIO decreases the F-actin signal intensity in chromosomally unstable cells. (a) Representative Western blots verify the depletion of CKAP5, EB1 and TRIO after siRNA-mediated knock downs. $\alpha$-tubulin was detected to serve as loading control. Quantification of actin cortex and cloud signal intensities of (b) SW620 (CIN) and (c) SW480 (CIN) cells demonstrating a decrease in actin signal intensity after knock down of CKAP5, EB1 or TRIO. Knock downs were archived by transfection with siRNA against indicated target genes. $48 \mathrm{~h}$ after transfection, cells were stained for $\mathrm{F}$ actin (AlexaFluor ${ }^{\mathrm{TM}} 594$ Phalloidin) and DNA (Hoechst33342). Box and whisker plots showing the median, quartile and range of the mean actin cortex and cloud intensity calculated from metaphase line scans of three independent experiments $(n=28-37$ cells per experiment, $t$-test). 
For the knock down of more downstream parts of the pathway, SW620, SW480 and HCT116 cells were transfected with siRNA for Rac1 and Arp3 and the knock down efficiency was proved by Western blots (Figure 3.20a). Again, SW620, SW480 and HCT116 cells showed a decrease in actin cortex and cloud signal intensity, whereby SW620 and SW480 cells showed a stronger overall reduction (up to $23 \%$ or $35 \%$ decrease in cortex signal and $26 \%$ or $37 \%$ in cloud signal intensity respectively) than HCT116 cells (up to $19 \%$ decrease in cortex and cloud signal intensity) (Figure 3.20b-d). These results confirm the findings from inhibitor treated cells described above, thereby further supporting the involvement of the introduced microtubule dynamic, EB1, TRIO, Rac1, Arp2/3 dependent pathway in the organization of the actin cytoskeleton.

a
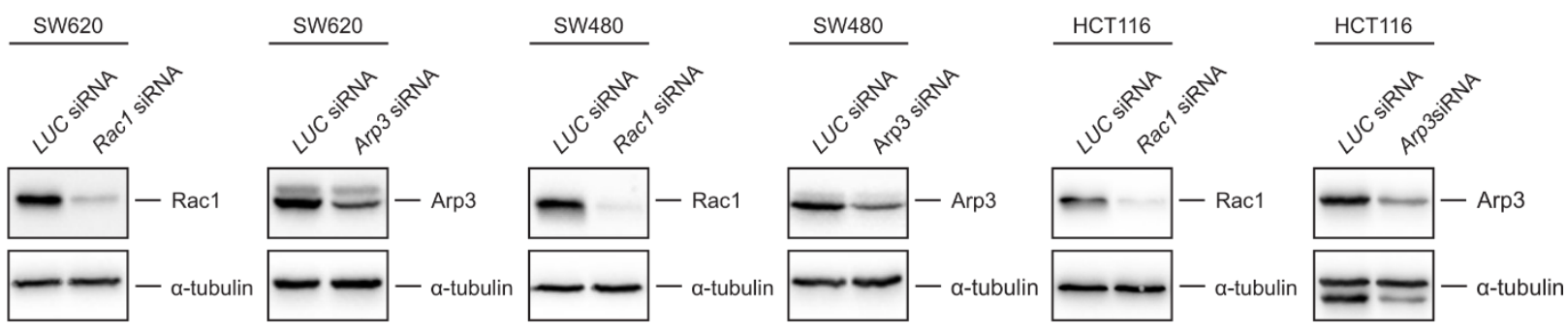

b
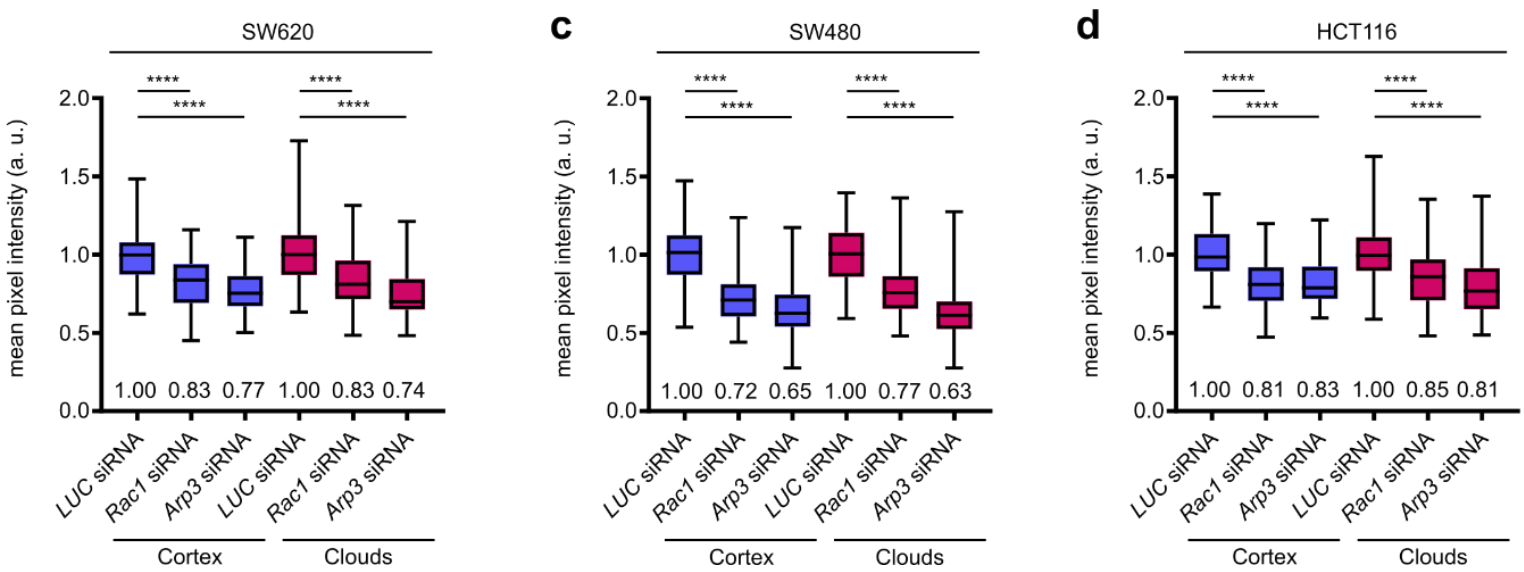

Figure 3.20: Knock down of Rac1 or Arp3 leads to a decrease in the F-actin signal intensity of chromosomally unstable and chromosomally stable cells. (a) Representative Western blots detecting Rac1 and Arp3 show the efficiency of siRNA-mediated knock downs of corresponding target genes. Detection of a-tubulin served as loading control. Actin cortex and cloud signal intensities of (b) SW620 (CIN), (c) SW480 (CIN) and (d) HCT116 (non-CIN) cells transfected with siRNA for Rac1 or Arp3 were quantified showing a more pronounced overall reduction for CIN cells. $48 \mathrm{~h}$ after siRNA-transfection, cells were stained for F-actin (AlexaFluor ${ }^{\mathrm{TM}} 594$ Phalloidin) and DNA (Hoechst33342). Box and whisker plots showing the median, quartile and range of the mean actin cortex and cloud intensity calculated from metaphase line scans of three independent experiments ( $n=20-37$ cells per experiment, $t$-test). 
Having shown that inhibition or downregulation of components of the given pathway causes a strong decrease in the F-actin signal intensity of chromosomally unstable cells, further experiments should now prove whether hyperactivation of pathway components would in turn lead to an increase in the F-actin signal intensity of chromosomally stable cells. Figuring out possible inductive conditions, treatment with low dose Nocodazole was chosen to induce increased microtubule plus-end assembly rates and overexpression of constitutively active Rac1 (Rac1-CA) was carried out for hyperactivation of the pathway further downstream (Figure 3.21a). However, neither low dose Nocodazole treatment, nor Rac1-CA overexpression resulted in an increase of actin cortex or cloud signal intensities (Figure 3.21b). A possible explanation for this result could be that increasing the activity of only one component of the pathway might not be enough to cause an hyperactivation of the whole pathway due to the still limited amount of downstream components. This is supported by the finding that TRIO is part of the HET70 signature of genes that are most strongly upregulated in cancers with karyotype heterogeneity (Sheltzer, 2013), which corresponds with Western blot results showing that TRIO expression was stronger in chromosomally unstable SW620 cells than in chromosomally stable HCT116 cells (Figure 3.5a).

a

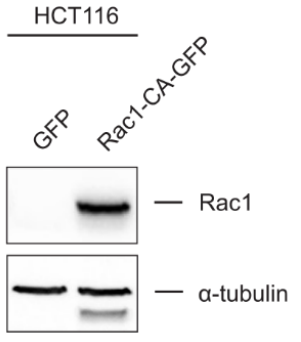

b

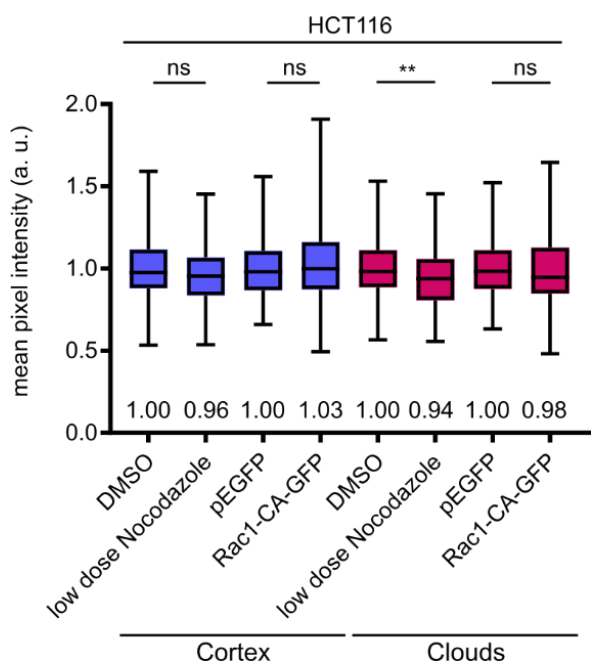

Figure 3.21: The F-actin signal intensity of chromosomally stable HCT116 cells is not affected by solely increasing microtubule plus-end assembly rates or by overexpression of constitutively active Rac1. (a) Representative Western blot showing overexpressed constitutively active Rac1 (Rac1-CA-GFP) in HCT116 cells. $\alpha$-tubulin was detected to serve as loading control. (b) Quantification of actin cortex and cloud signal intensities showing no impact of low dose Nocodazole or overexpression of Rac1-CA-GFP in HCT116 cells. Cells were transfected with a plasmid encoding Rac1-CA-GFP or GFP as control $48 \mathrm{~h}$ before fixation or treated with DMSO (control) or $0.5 \mathrm{nM}$ Nocodazole $16 \mathrm{~h}$ before fixation, followed by staining for F-actin (AlexaFluor TM 594 Phalloidin) and DNA (Hoechst33342). Box and whisker plots showing the median, quartile and range of the mean actin cortex and cloud intensity calculated from metaphase line scans of five to six independent experiments ( $n=26-35$ cells per experiment, $t$-test). 
These findings indicate that an hyperactivation of the given pathway requires not only an increase in microtubule plus-end assembly rates, but also a higher expression of TRIO. However, this might not serve as possible explanation for the observed result from constitutively active Rac1 overexpressing HCT116 cells, since Rac1 is downstream of TRIO. Here, the correct localization of activated Rac1 might again be the crux of the matter, meaning that only overexpressing one pathway component does not automatically result in this component being localized at the required site of action. Consequently, for a successful hyperactivation of the introduced pathway it seems to be necessary that all components involved are active at the correct location. This would need to be proved in further experiments.

The results so far indicate that the F-actin cytoskeleton is indeed regulated by the introduced pathway in chromosomally unstable cells. However, regulation of the mitotic F-actin cytoskeleton was mainly described to be dependent on other pathways, whereby RhoA dependent pathways need to be particularly mentioned (Maddox \& Burridge, 2003). RhoA activity increases at the onset of mitosis and leads to activation of its effectors, especially the Rho-associated kinase ROCK and the formin mDia. ROCK activates myosin II via phosphorylation of the myosin light chain (MLC), thereby increasing actin binding of myosin II which results in enhanced contractility (Amano et al., 1996). The formin mDia is a well-known actin nucleator, but instead of branched F-actin that is generated by Arp2/3 dependent actin nucleation mDia promotes nucleation of unbranched F-actin (Goode \& Eck, 2007). The RhoA effectors ROCK and mDia were both reported to be involved in mitotic cortex contractility and cytokinesis (Bovellan et al., 2014; Kosako et al., 2000; Maddox \& Burridge, 2003). Since RhoA and its effectors play an important role in the regulation of the mitotic F-actin cytoskeleton, it was of interest whether such other actin regulating pathways would also be involved in spindle axis alignment, chromosome segregation and the development of chromosomal instability. In order to investigate this question, SW620 and HCT116 cells were treated with different inhibitors and activators for RhoA and its effectors, followed by determining their effect on F-actin signal intensities. To inhibit RhoA, the C3 toxin was used, whereas calpeptin treatment was chosen for RhoA activation. Further downstream, formins were inhibited by SMIFH2. However, only very low concentrations of calpeptin and SMIFH2 were used, as work from our group has been shown that these already affect spindle positioning and the generation of lagging chromosomes (Schermuly, 2019). Additionally, Latrunculin A was used for general promotion and Jasplakinolide for general inhibition of F-actin assembly. While inhibition of RhoA by $\mathrm{C} 3$ resulted in disruption of a functional mitotic $\mathrm{F}$-actin cortex that was even more pronounced In SW620 cells than in HCT116 cells (Figure 3.22a), an activation of RhoA by calpeptin treatment did not lead to obvious changes in the F-actin cytoskeleton and its signal intensity (Figure 3.22a- 
b). Inhibition of formins by SMIFH2 treatment did also not cause visible changes in the F-actin cytoskeleton and in its signal intensity (Figure 3.22a-b). Cells treated with the F-actin assembly promoting drug Jasplakinolide seemed to have an intact actin cortex but showed the formation of massive subcortical F-actin aggregates (Figure 3.22a). Interestingly, treatment with the F-actin

a

DMSO

$3 \mu \mathrm{g} / \mathrm{ml} \mathrm{C} 3$ toxin

RhoA inhibitor

$1 \mu \mathrm{g} / \mathrm{ml}$ Calpeptin RhoA activator

$20 \mu \mathrm{M}$ SMIFH2 Formin inhibitor

$0.1 \mu \mathrm{M}$ Jasplakinolide promotes F-actin polymerization

$0.75 \mu \mathrm{M}$ Latrunculin A prevents F-actin assembly

C
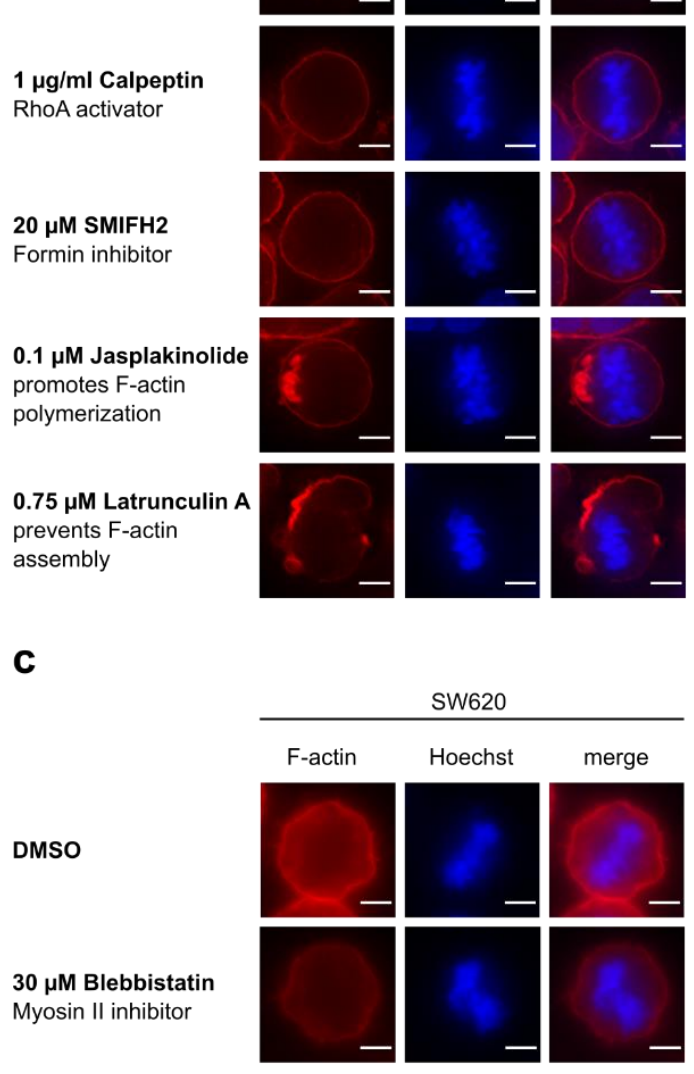

b
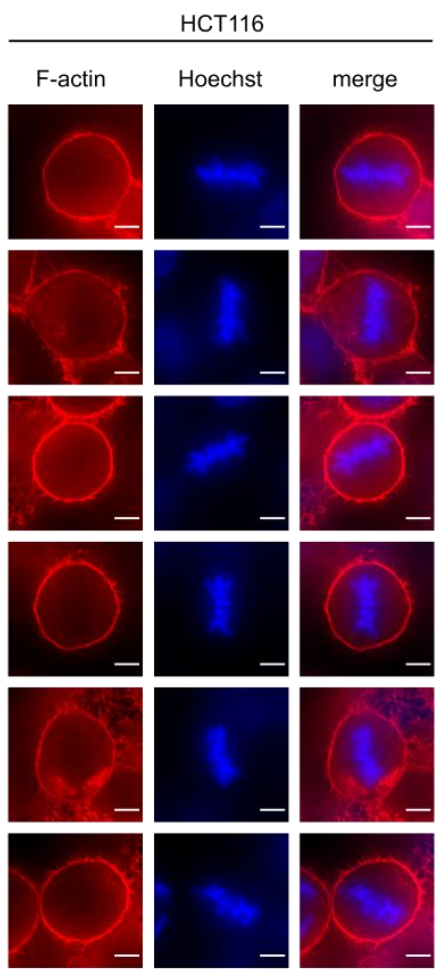

d

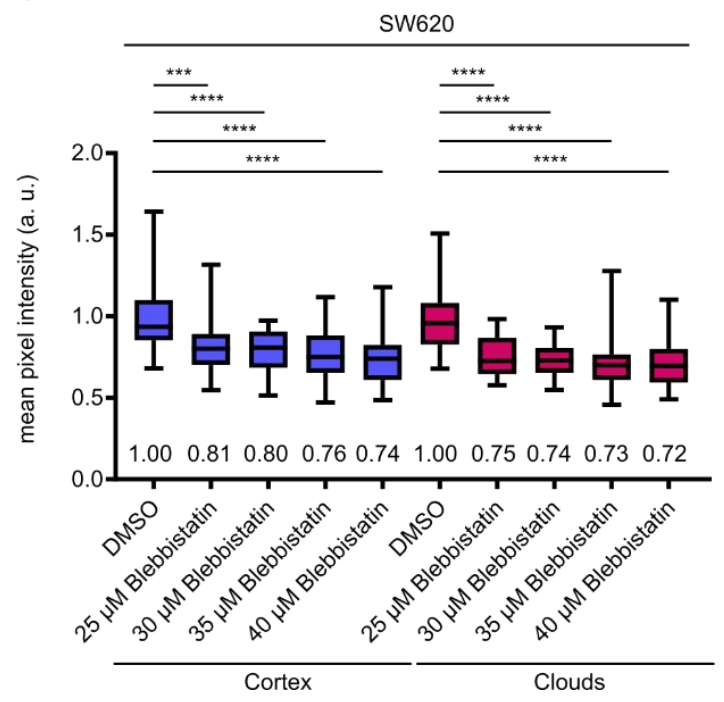


Figure 3.22: The F-actin signal intensity of chromosomally unstable SW620 cells is not affected by mild activation of RhoA or mild inhibition of formins, but by inhibition of RhoA or myosin II. (a) Representative immunofluorescence images of SW260 and HCT116 cells after treatment with drugs affecting RhoA or its downstream effectors or drugs directly affecting F-actin assembly. Cells were treated with DMSO (control), $3 \mu \mathrm{g} / \mathrm{ml} \mathrm{C3}$ toxin (RhoA inhibitor) for $6 \mathrm{~h}, 1 \mu \mathrm{g} / \mathrm{ml}$ calpeptin (RhoA activator) for $2 \mathrm{~h}$, $20 \mu \mathrm{M}$ SMIFH2 (formin inhibitor) for $2 \mathrm{~h}, 0.1 \mu \mathrm{M}$ Jasplakinolide (inducer of F-actin assembly) for $16 \mathrm{~h}$ or $0.75 \mu \mathrm{M}$ Latrunculin A (inhibitor of F-actin assembly) for $2 \mathrm{~h}$ and stained for F-actin (AlexaFluor ${ }^{\mathrm{TM}} 594$ Phalloidin; red) and DNA (Hoechst33342; blue). Scale bar $5 \mu \mathrm{m}$. (b) Quantification of actin cortex and cloud signal intensities of SW620 cells showing no effect after RhoA activation or formin inhibition. Cell were treated with calpeptin or SMIFH2 and stained as described in (a). Box and whisker plots showing the median, quartile and range of the mean actin cortex and cloud intensity calculated from metaphase line scans of three independent experiments ( $n=20-24$ cells per experiment, $t$-test). (c) Representative immunofluorescence images of SW260 cells treated with the myosin II inhibitor blebbistatin showing a clear decrease in F-actin signal intensity. Cells were treated with DMSO or $30 \mu \mathrm{M}$ blebbistatin for $1 \mathrm{~h}$ and stained as described in (a). Scale bar $5 \mu \mathrm{m}$. (d) Actin cloud and cortex signal intensities from SW620 cells treated with DMSO or with $25,20,35$ or $40 \mu \mathrm{M}$ blebbistatin for $1 \mathrm{~h}$ were quantified and show a concentration dependent decrease. Cells were stained as described in (a). Box and whisker plots showing the median, quartile and range of the mean actin cortex and cloud intensity calculated from metaphase line scans of one blebbistatin titration experiment $(n=27-32$ cells per different concentration, $t$-test).

assembly inhibiting drug Latrunculin A lead to a strong disruption of the F-actin cortex in mitotic SW620 cells, but caused almost no visible effects in mitotic HCT116 cells (Figure 3.22a). When trying to figure out the meaning of these results, the cortical F-actin disrupting (SW620) or diminishing (HCT116) effect of RhoA inhibition is not surprising, as Nishimura et al. (2019) also detected a decreased enrichment of cortical F-actin in C3 treated metaphase Hela cells. RhoA inhibition results in reduced activation of its effector ROCK. Less active ROCK leads to less active myosin II, thereby causing a reorganization of cortical F-actin from dense bundles to a loose, disorganized F-actin network (Ivanov et al., 2008). This might explain the observed changes in the F-actin structure after C3 treatment. However, the formin mDia1, an isoform of mDia, is also an effector of RhoA and known to be a major contributor to nucleation of cortical actin (Bovellan et al., 2014), therefore raising the question why inhibition of formins by SMIFH2 did not cause visible changes in the F-actin cortex or its signal intensity (Figure 3.22Figure 3.22a-b). Interestingly, while Bovellan et al. (2014) also showed a drastic reduction in F-actin cortex fluorescence intensity after Arp2/3 inhibition by CK666, they only detected a slight, but not significant reduction after mDia1 knock down. Despite this obvious difference on F-actin cortex intensity between Arp2/3 and mDia1 downregulation, their work revealed a more important role for mDia1 than for Arp2/3 in cell division. This suggests that although F-actin cortex signal intensities can be determined to reveal changes in cortical F-actin, it cannot be used to show all changes that might appear. This might also be true for changes induced by activation of RhoA by calpeptin treatment as it did not increase F-actin cortex signal intensities (Figure 3.22a-b). At this point, it should also be noted, that the used concentrations of calpeptin and SMIFH2 were quite low when compared to literature, thereby raising the possibility that higher concentrations might cause a measurable effect. 
In order to prove whether the RhoA effector ROCK and its target myosin II are responsible for the observed disruption of the F-actin cytoskeleton, SW620 cells were treated with the myosin II inhibitor blebbistatin. F-actin signal intensity measurements clearly showed a concentration dependent decrease after myosin II inhibition (Figure 3.22c-d), thereby supporting the observed changes in the cortical actin structure reported by Ivanov et al. (2008). The range of the detected decrease was comparable with the measured F-actin signal intensities after inhibition of the EB1, TRIO, Rac1, Arp2/3 dependent pathway. Therefore, it would be worth investigating whether an inhibition of the RhoA dependent pathway and especially myosin II would also cause a rescue of chromosomal instability. However, the results concerning the RhoA-related pathway also demonstrate that only measuring the F-actin signal intensity is not sufficient to detect every possible change in the F-actin structure. For this reason, other experimental approaches are required that allow further insight into the F-actin structure and its physical properties.

\subsection{Chromosomally unstable cells exhibit decreased mitotic actin cortex tension that is rescuable by inhibition of a microtubule dynamic, EB1, TRIO, Rac1, Arp2/3 dependent pathway}

Although measurements of the mitotic F-actin signal intensity revealed a role for the introduced microtubule dynamic, EB1, TRIO, Rac1, Arp2/3 dependent pathway in the regulation of the F-actin cytoskeleton especially in chromosomally unstable cells, further experimental approaches were required to figure out what consequences the observed changes have for a mitotic cell. Recent studies revealed that cortical tension is higher in mitotic than in interphase cells (Fischer-Friedrich et al., 2014; Stewart et al., 2011), and that not myosin II activity alone but also the actin cortex architecture itself is involved in the generation of cortical tension (Chugh et al., 2017). Moreover, cortical rigidity and tension has been reported to regulate spindle positioning in mitotic cells (Kozlowski et al., 2007; Morin \& Bellaïche, 2011). These findings led to the suggestion that the observed changes in F-actin structure and signal intensity due to interfering with the given pathway might have an impact on the mitotic cortex tension, thereby regulating mitotic spindle positioning. In order to test this assumption, atomic force microscopy (AFM) indentation experiments were performed on chromosomally stable HCT116 and chromosomally unstable SW620 cells in prometaphase that have been treated with drugs affecting the given pathway. Interestingly, HCT116 cells exhibited a generally higher cortex tension when compared to SW620 cells (Figure 3.23a), thereby indicating that chromosomally unstable cells have a decreased mitotic cortex tension. This was further supported by the finding that the cortex tension of SW620 cells 
increased, and thus were rescued to HCT116 level by restoring proper microtubule plus-end assembly rates using low dose Taxol or by inhibiting TRIO, Rac1 or Arp2/3 (Figure 3.23). In contrast, treatment of HCT116 cells with low dose Taxol or with a Rac1 inhibitor did not affect cortex tension. Consequently, HCT116 cells seem to already have an optimal mitotic cortex tension that cannot be further increased by the given treatments. Interestingly, low dose Nocodazole treatment did almost not affect the cortex tension of mitotic HCT116 cells, thereby supporting the result from F-actin signal intensity measurements that also did not show an effect for Nocodazole (Figure 3.21b).

a

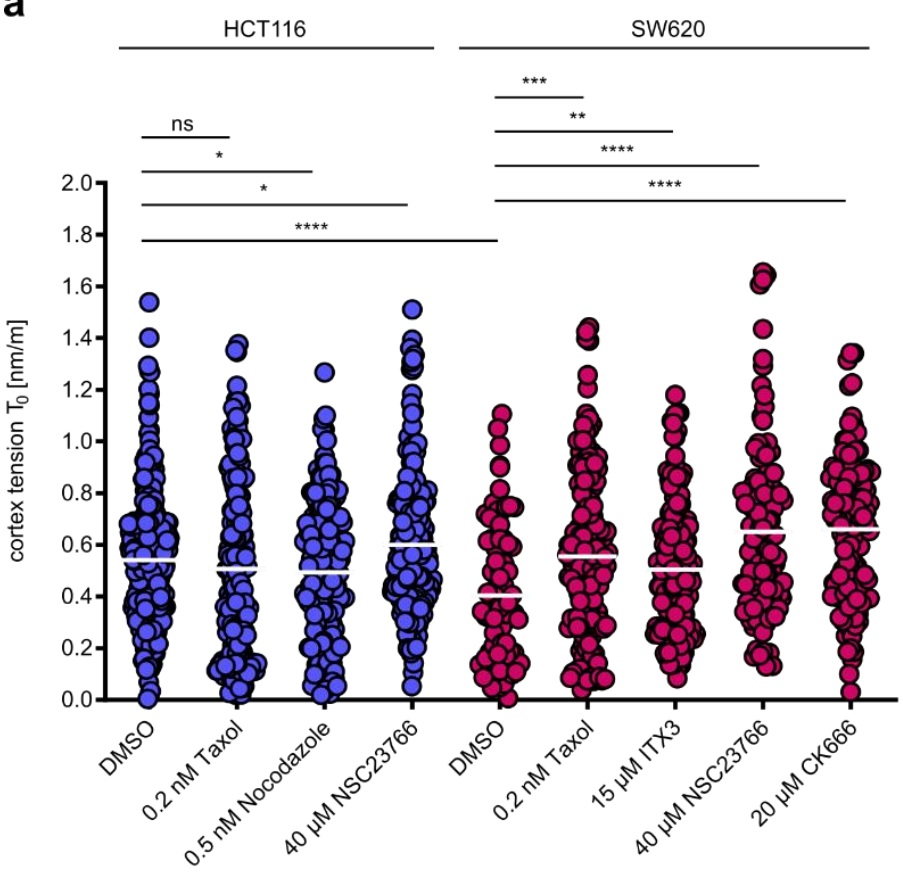

b

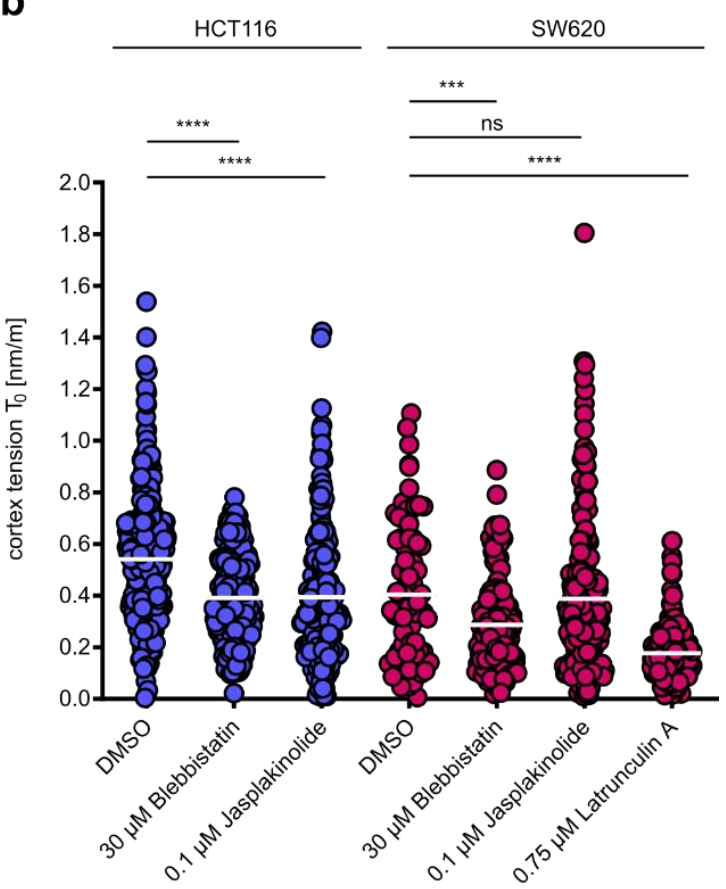

Figure 3.23: Chromosomally unstable SW620 cells exhibit decreased mitotic cortex tension $T_{0}$ that is rescuable by inhibition of the microtubule dynamic, EB1, TRIO, Rac1, Arp2/3 dependent pathway, but not by myosin II inhibition. (a) AFM indentation measurements of prometaphase HCT116 (non-CIN) and SW620 (CIN) cells showing a decreased cortex tension for CIN cells and its rescue by Taxol-induced restoration of proper microtubule plus-end assembly rates or inhibition of TRIO, Rac1 or Arp2/3. Cells were transfected with a GFP-H2B expressing plasmid to allow for identification and measurement of prometaphase cells and treated with DMSO (control), $0.2 \mathrm{nM}$ Taxol, $0.5 \mathrm{nM}$ Nocodazole, $15 \mu \mathrm{M}$ ITX3 (TRIO inhibitor), $40 \mu \mathrm{M}$ NSC23766 (Rac1 inhibitor) or $20 \mu \mathrm{M}$ CK666 (Arp2/3 inhibitor) for about $16 \mathrm{~h}$. Scatter dot plots show calculated values for cortical tension $(n \geq 20$ cells in at least three independent experiments, mean \pm SEM, $t$-test). (b) Measurements of cortex tension of prometaphase HCT116 and SW620 cells showing a decreasing effect for myosin II inhibition, as well as by increasing or decreasing F-actin assembly. Cells were transfected as described in (a) and treated with DMSO, $30 \mu \mathrm{M}$ blebbistatin (myosin II inhibitor) for $1 \mathrm{~h}, 0.1 \mu \mathrm{M}$ Jasplakinolide (inducer of F-actin assembly) for $16 \mathrm{~h}$ or $0.75 \mu \mathrm{M}$ Latrunculin A (inhibitor of F-actin assembly) for $2 \mathrm{~h}$. Scatter dot plots show calculated values for cortical tension ( $\mathrm{n} \geq 20$ cells in at least three independent experiments, mean \pm SEM, $t$-test). AFM indentation measurements were carried out in collaboration with Dr. Brückner and Prof. Dr. Janshoff (Institute for Biophysical Chemistry, Georg August University Göttingen) and Nadine Schermuly (Institute for Molecular Onkology, University Medical Center Göttingen) with distribution of tasks described in chapter 2.2.14. Nadine Schermuly already presented the data for DMSO, Taxol and blebbistatin treatment in her dissertation (Schermuly, 2019). 
Inhibition of myosin II by blebbistatin treatment has shown a similar decrease in F-actin signal intensity as drugs affecting the given actin-regulating pathway (Figure 3.22c-d). However, blebbistatin is known to reduce cortical tension (Chugh et al., 2017), which has been confirmed by measuring the cortex tension of HCT116 and SW620 cells after blebbistatin treatment (Figure 3.23b). These opposite effects on cortical tension suggest that although showing the same decreasing effect in F-actin signal intensity, inhibition of myosin II seems to induce different changes in the mitotic F-actin cortex structure than inhibition of the microtubule dynamic, EB1, TRIO, Rac1, Arp2/3 dependent pathway. In order to further investigate the effect of a general increase or decrease in F-actin assembly, HCT116 and SW620 cells were treated with Jasplakinolide or Latrunculin A. Increased F-actin assembly by Jasplakinolide treatment caused a clear decrease in cortex tension in HCT116 cells to approximately untreated SW620 level, whereas the cortex tension of SW620 did not further decrease (Figure 3.23b). In contrast, inhibition of F-actin assembly by treatment with Latrunculin A made it possible to reduce the cortex tension in SW620 cells even further. These observed decrease in cortex tension might also be explained by the severe changes that have been observed for the mitotic F-actin structure (Figure 3.22a).

Taken together, AFM indentation measurements revealed a link between the microtubule dynamic, EB1, TRIO, Rac1, Arp2/3 dependent pathway and the generation of mitotic cortex tension. It could be shown that chromosomally unstable cells exhibit a decreased mitotic cortex tension that is rescuable by inhibition of components of the introduced pathway, thereby probably enabling a proper spindle axis alignment and chromosome segregation.

Abovementioned results show that cortical tension depends on actin cortex architecture, whereby measuring the F-actin signal intensity is considered to represent actin density. Interestingly, Chugh et al. (2017) found out, that also the actin cortex thickness plays an important role for the generation of mitotic cortex tension. In order to investigate whether there is a correlation between actin cortex thickness and actin cortex density, the actin cortex thickness of SW620 cells, in which the introduced microtubule plus-end-EB1-TRIO-Rac1-Arp2/3 pathway was downregulated, were determined. Therefore, the cells were treated with low dose Taxol, the TRIO inhibitor ITX3, the Rac1 inhibitor NSC23766 or the Arp2/3 inhibitor CK666. Interestingly, there was no effect on cortex thickness by any of the treatments (Figure 3.24a). This shows, that either a change in actin cortex density or in actin cortex thickness is sufficient to affect cortex tension. Moreover, this result is consistent with the finding from Chugh et al. (2017), who also did not detect a change in actin 

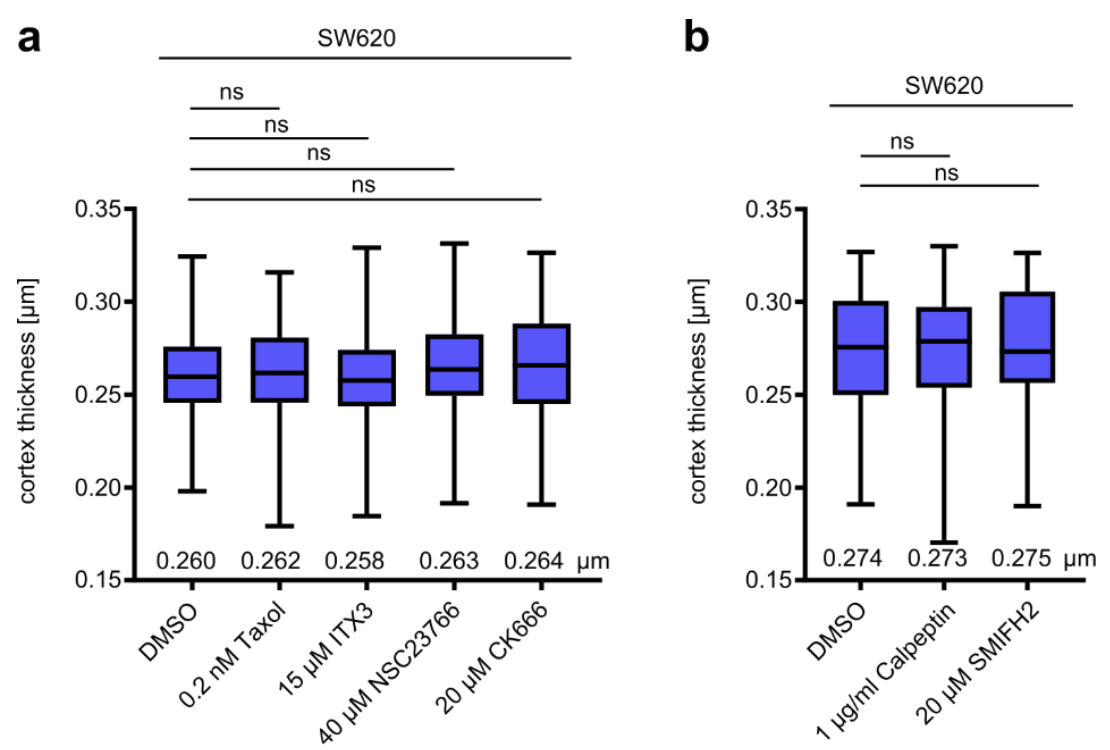

Figure 3.24: The actin cortex thickness of is not affected by downregulation of components of the microtubule plus-end-EB1-TRIO-Rac1-Arp2/3 pathway. (a) Measurements of actin cortex thickness of metaphase SW620 cells (from Figure 3.18c) showing no effect for a Taxol-induced rescue of microtubule plus-end growth rates or inhibition of TRIO, Rac1 or Arp2/3. Cells were treated with DMSO, $0.2 \mathrm{nM}$ Taxol, $15 \mu \mathrm{M}$ ITX3, $40 \mu \mathrm{M}$ NSC23766 or $20 \mu \mathrm{M}$ CK666 for $16 \mathrm{~h}$ and stained for F-actin (AlexaFluor 594 Phalloidin) and DNA (Hoechst33342). Box and whisker plots showing the median, quartile and range of the mean actin cortex thickness calculated from metaphase cells of three independent experiments $(n=20-36$ cells per experiment, $t$-test). (b) Actin cortex thickness measurements of metaphase SW620 cells (from Figure 3.22b) demonstrating no effect for slightly increased RhoA activation or slightly reduced formin inhibition. Cells were treated with DMSO, $1 \mu \mathrm{g} / \mathrm{ml}$ calpeptin (RhoA activator) for $2 \mathrm{~h}$ or $20 \mu \mathrm{M} \mathrm{SMIFH} 2$ (formin inhibitor) for $2 \mathrm{~h}$ and stained as described in (a). Box and whisker plots showing the median, quartile and range of the mean actin cortex thickness calculated from metaphase cells of three independent experiments $(n=20-24$ cells per experiment, $t$-test $)$.

cortex thickness after Arp2/3 inhibition by CK666, but only after changing actin filament length regulators, such as $\mathrm{mDia}$, capping protein or cofilin. Since mDia belongs to the family of formins and is also regulated by RhoA, the question was raised whether SW620 cells treated with calpeptin or SMIFH2, which did not alter F-actin cortex intensity, would rather show changes in the actin cortex thickness. However, the low concentrations used did not cause alterations in actin cortex thickness (Figure 3.24b).

Due to the finding that measurement of the mitotic F-actin signal intensity is not sufficient to detect every change in the F-actin structure, and that in case of a change it gives no information whether this induces an increase or decrease of cortex tension, additional experimental approaches should enable visualization of extremely fine structural alterations in the F-actin cytoskeleton. Therefore, STED (stimulated emission depletion) microscopy as one of the techniques to create super- 
Results

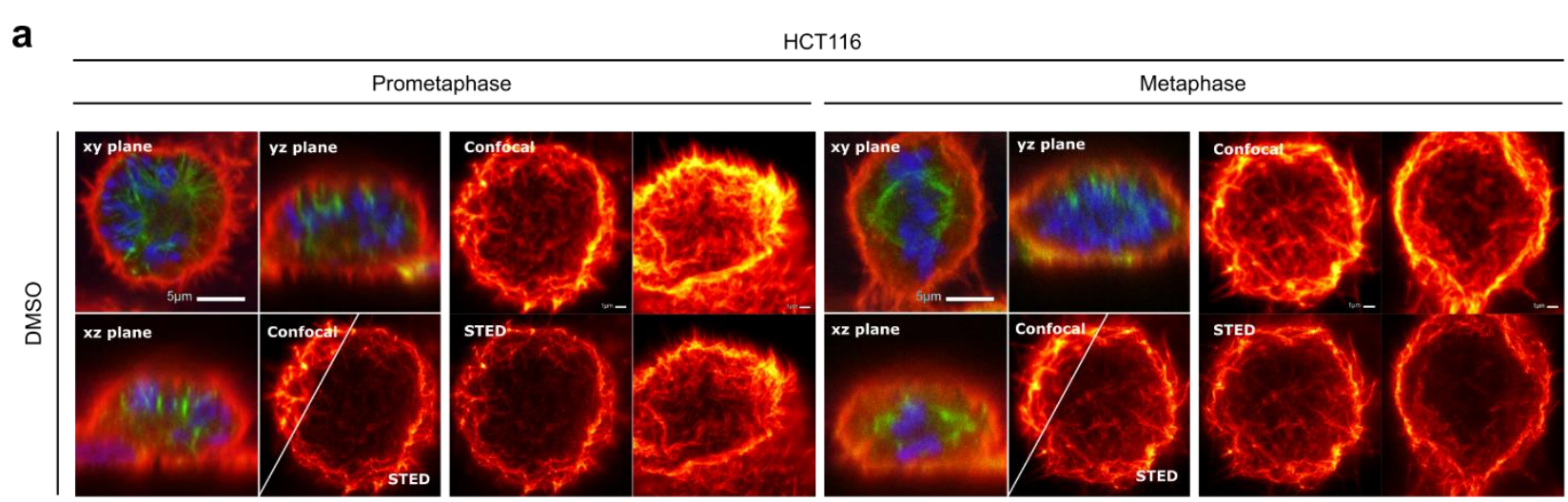

b

SW620
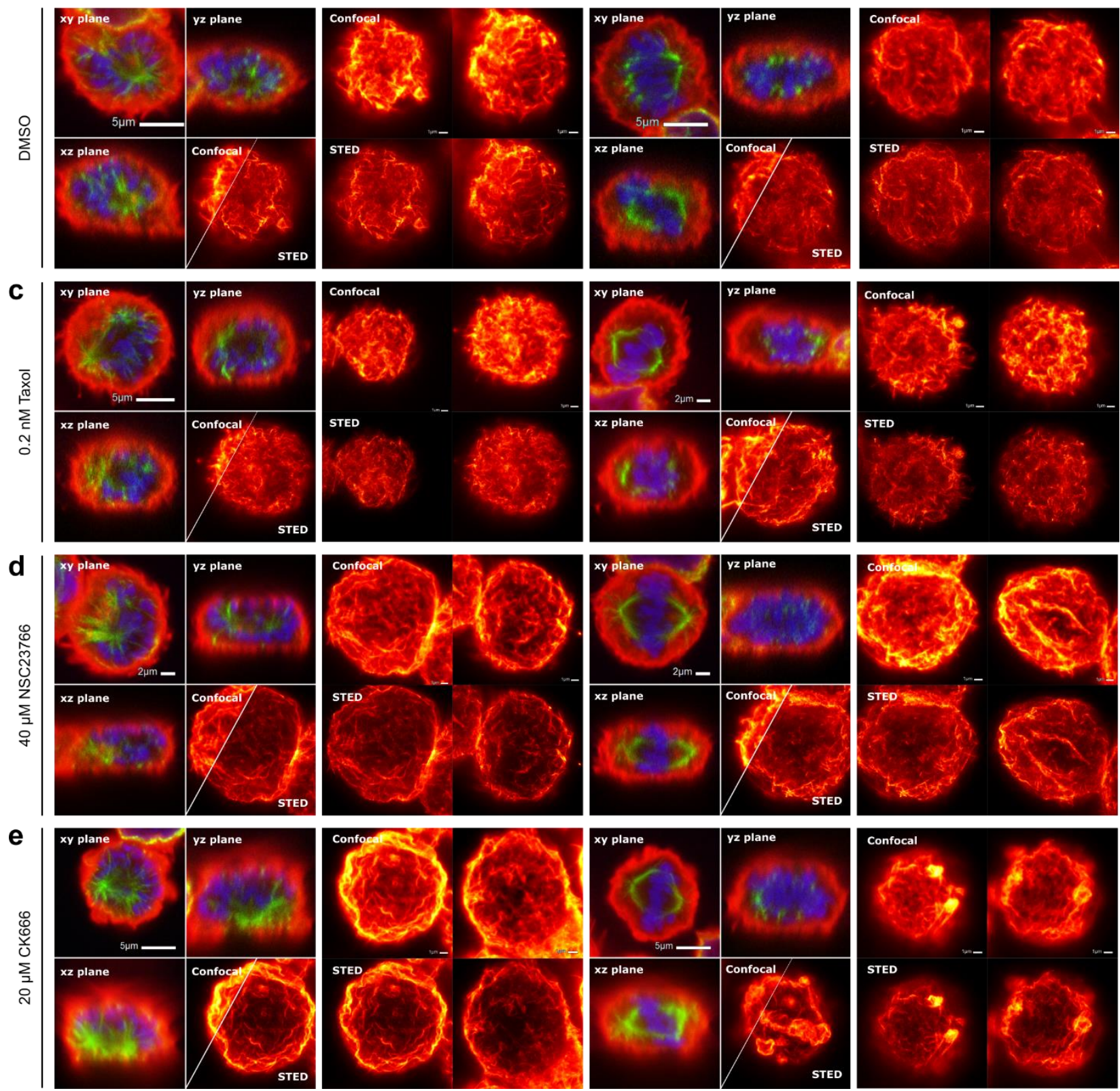
Figure 3.25: STED microscopy visualizes changes in the F-actin cytoskeleton of mitotic SW620 cells after inhibition of Rac1 and Arp2/3, but not after Taxol-induced restoration of microtubule plus-end assembly rates. Representative three color confocal images of DNA (blue), $\alpha$-tubulin (green) and F-actin (red) in xy-, xz- and yz plane, respectively, as well as confocal and corresponding STED images of the top surface F-actin network of (a) DMSO treated HCT116 cells, and SW620 cells treated with (b) DMSO, (c) $0.2 \mathrm{nM}$ Taxol, (d) $40 \mu \mathrm{M}$ NSC23766 (Rac1 inhibitor) or (e) $20 \mu \mathrm{M}$ CK666 (Arp2/3 inhibitor). Cells were seeded on glass coverslips, treated with the indicated drugs for $16 \mathrm{~h}$ and stained for $\mathrm{F}$-actin (Abberior ${ }^{\circledR}$ STAR-RED Phalloidin; red), $\alpha$-tubulin (Abberior ${ }^{\circledR}$ STAR 580; green) and DNA (Hoechst33342; blue). Scale bars as indicated. Maximum projections of top surface images of $15 \mathrm{z}$-stacks with a z-optical spacing of $0.2 \mu \mathrm{m}$ are shown. STED microscopy was carried out in collaboration with Florian Grimm (Abberior $\mathrm{GmbH}$, Göttingen).

resolution images was performed. HCT116 and SW620 cells were grown on glass coverslips and microtubule structures were labelled with Abberior ${ }^{\circledR}$ STAR 580, whereas F-actin was stained with Abberior $^{\circledR}$ STAR-RED Phalloidin. When comparing STED images of the top surface of mitotic (prometaphase or metaphase) cells, HCT116 cells clearly exhibited a more defined cortical Factin structure (Figure 3.25a), whereas SW620 cells showed a rather diffuse cortical F-actin network that seemed to strongly pervade cytoplasmic regions (Figure 3.25b). For restoration of proper microtubule plus-end assembly rates, SW620 cells were treated with low dose Taxol and for inhibition of Rac1 or Arp2/3 with NSC23766 or CK666, respectively. However, while inhibition of Rac1 or Arp2/3 indeed induced the formation of a rather defined F-actin cortex similar to but still not as defined as in HCT116 cells, low dose Taxol treatment did not lead to visible changes of the F-actin cytoskeleton (Figure 3.25c-e). Interestingly, Arp2/3 inhibition additionally induced the formation of actin-rich blebs at the cortex, which was detected especially in metaphase SW620 cells (Figure 3.25e) and has also been observed from Bovellan et al. (2014). These observed differences in the effect of the given treatments suggest that although a general inhibition of Rac1 or Arp2/3 indeed triggers remodeling of the F-actin cytoskeleton, these visible changes might not be due to a downregulation restricted to the microtubule dynamic, EB1, TRIO, Rac1, Arp2/3 pathway. There might be even more subtle changes in the F-actin cytoskeleton caused by interference with this pathway that are not even visible by STED microscopy. 


\section{Discussion}

Recently, evidence came up by work from our group, that there is a new pathway that provides a link between microtubule dynamics and the mitotic actin cortex. When hyperactivated, this pathway seems to induce the generation of spindle mis-positioning, chromosome mis-segregation and thereby chromosomal instability (Berger, 2016; Schermuly, 2019). Moreover, in interphase cells this pathway was also shown to be involved in cell migration (Berger, 2016). From recent work it is known that the pathway includes the microtubule plus-end binding protein EB1, the GEF TRIO and its Rho GTPase Rac1, as well as the F-actin regulating Arp2/3 complex. In addition, increased mitotic microtubule plus-end growth rates, as exhibited by chromosomally unstable cancer cells, were associated with a hyperactivation of this pathway. However, many links within this pathway were still elusive and required further investigation.

This study revealed a microtubule dynamic dependent EB1-TRIO interaction at microtubule plus ends in mitotic cells, that is required for downstream activation of Rac1 and Arp2/3, thereby causing cortical actin cytoskeleton reorganization. When hyperactivated, such as by increased microtubule plus end assembly rates found in CIN cells, this pathway triggers the formation of an actin cortex architecture with increased density that causes decreased cortex tension. A reduced cortical tension, in turn, is associated with defects in spindle positioning, as demonstrated by Kunda et al. (2008), and this was further shown to cause chromosome mis-segregation and thus CIN (Ertych et al., 2014; Silkworth \& Cimini, 2012). Moreover, this study is the first to show that an increase in the level and thus the activity of the Rho GEF TRIO triggers EB1 binding dependent whole and structural chromosome instability as well as EB1 binding independent structural chromosome instability. Moreover, whole chromosome instability could be rescued by downregulation of TRIO, Rac1 or Arp2/3, thereby confirming Rac1 as the actual TRIO effector within this pathway. However, structural chromosome instability could only partially be rescued by downregulation of the TRIO-Rac1-Arp2/3 pathway. This again confirms that there is an additional EB1 independent pathway participating in the generation of structural chromosome instability, driven by another TRIO effector than Rac1. Additionally, this study revealed that the increase in cell migration observed for cells with enhanced TRIO levels and activity is not only EB1 binding independent, but there is even a stronger increase in cell migration for cells exhibiting high levels of active EB1 binding deficient TRIO. Summing up all unraveled TRIO functions, this protein drives EB1 dependent whole and structural chromosome instability, EB1 independent structural chromosome instability and EB1 binding independent cell migration via different effector pathways (Figure 4.1). 


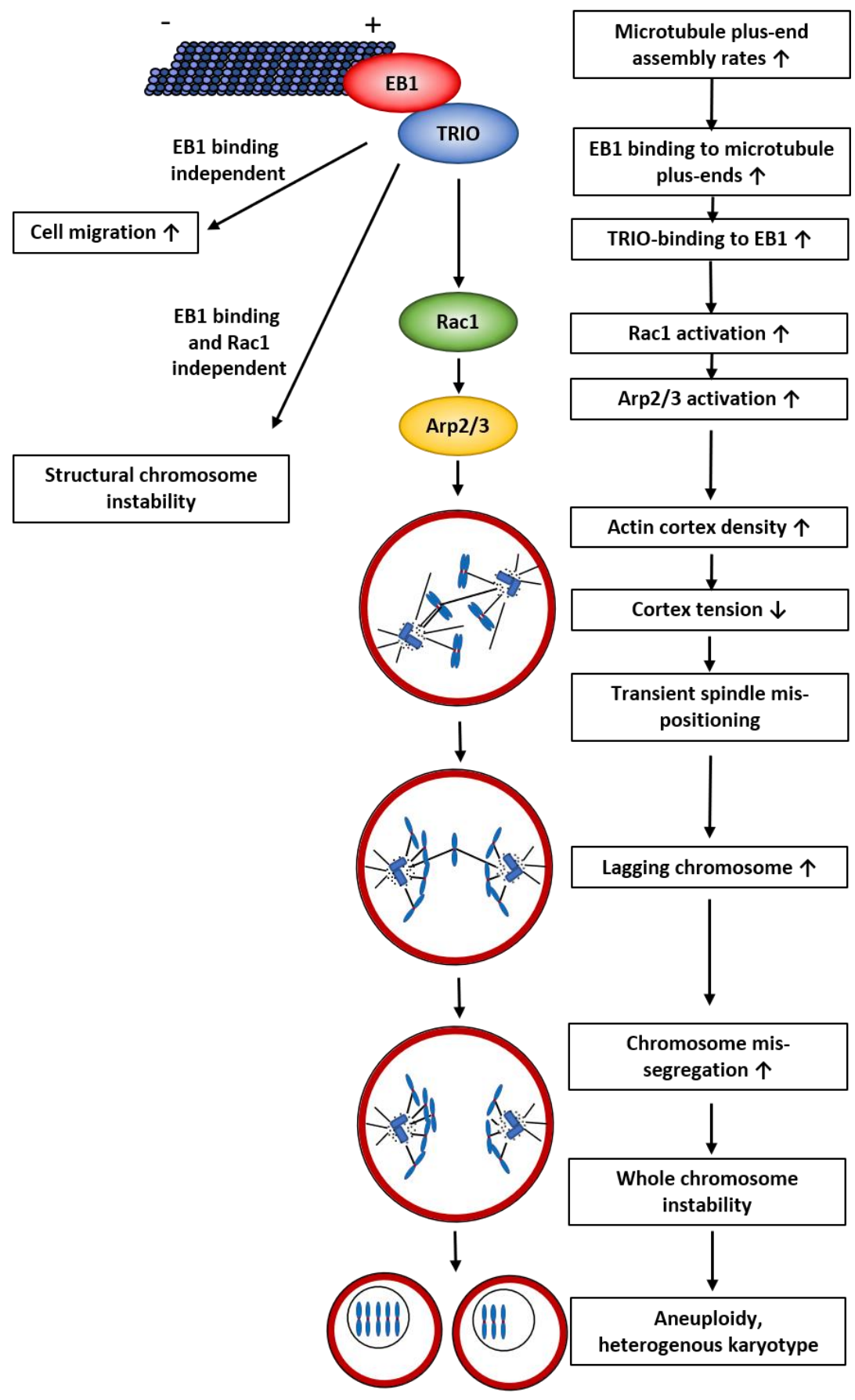

Figure 4.1: Model for the microtubule plus-end-EB1-TRIO-Rac1-Arp2/3 dependent pathway and its role in the development of CIN via decreasing actin cortex tension. An irregular increase in mitotic microtubule plus-end growth rates results in hyperactivation of a pathway involving EB1, TRIO, Rac1 and the F-actin organizing Arp2/3 complex. This leads to an increase in actin cortex connectivity and thus density, thereby causing a decreased cortical tension. A low cortex tension in turn results in a transient defect of the mitotic spindle position and thereby triggers the formation of merotelic kinetochore-microtubule attachments, followed by an increased amount of lagging chromosomes. Consequently, a higher rate of chromosome mis-segregation occurs that finally leads to whole chromosome instability. Additionally, upregulated TRIO also induces structural chromosome instability in an EB1 and Rac1 independent pathway. Moreover, upregulated TRIO also increases cell migration in an EB1 independent manner. 


\subsection{Why is the TRIO-EB1 interaction affected by a Taxol dependent decrease, but not by a Nocodazole independent decrease of microtubule plus-end assembly rates?}

Immunofluorescence images clearly demonstrated an EB1 dependent localization of TRIO to microtubule plus-ends. Although affecting microtubule dynamics did not visibly reduce the amount of TRIO localized to microtubule plus-ends, further pull down experiments revealed a decrease in TRIO-EB1 interaction of chromosomally unstable HEK293T cells due to a Taxol induced decrease of microtubule plus-end growth rates. This observed reduction of TRIO-EB1 binding is consistent with the finding, that EB1 preferentially binds to growing microtubule plus ends (Vaughan, 2005): Due to low dose Taxol treatment microtubule dynamics are especially reduced at microtubule plus-ends (Derry et al., 1998), that also means a decrease in microtubule plus-end growth rates (Ertych et al., 2014), thereby resulting in diminished EB1 binding to microtubule plus-ends. Consequently, EB1 recruitment and interaction with other + TIP proteins, such as TRIO, is diminished. While in this study, a Taxol induced decrease in microtubule plus-end dynamics was observed to diminish the interaction of TRIO and EB1, work from van Haren et al. (2014) demonstrated, that stabilization of microtubules by high doses of Taxol affects the interaction between TRIO and the +TIP protein Nav1 and causes depletion of the TRIO-Nav1 complex at neurite tips. Both low and high doses of Taxol reduce microtubule dynamics and especially plusend growth rates, thereby triggering the disassembly of +TIPs, such as TRIO, Nav1 and EB1, at microtubule plus ends.

In order to prove, whether an increase in microtubule plus-end growth rates of chromosomally stable HCT116 cells would have the opposite effect, low dose Nocodazole treatment was used, as this has been frequently shown to increase microtubule growth rates in our group (Berger, 2016; Ertych et al., 2014; Schermuly, 2019). However, there was no increase in TRIO-EB1 binding following low dose Nocodazole treatment. Interestingly, Nocodazole was found to not only bind to the colchicine binding site at the $\beta$-tubulin subunit of tubulin dimers, but also of microtubule polymers (Lee et al., 1980). Additionally, Nunez et al. (1979) found out, that binding of colchicine to tubulin and binding of microtubule associated proteins is competitive. Although Nunez et al. (1979) did not further specify the affected microtubule associated proteins, it might be possible, that Nocodazole competes with EB1 for tubulin binding. This would cancel out the positive effect, that a Nocodazole induced increase in microtubule dynamics might have on EB1 binding to growing microtubule plus ends. If this would be the case, another explanation than the EB1-TRIORac1-ARP2/3 dependent pathway would be required to explain the observed inducing effects of low dose Nocodazole treatment on spindle mis-orientation, chromosome mis-segregation and karyotype variability, found by Ertych et al. (2014). Jordan et al. (1992) also found low dose 
Nocodazole to affect mitotic spindle morphology, as microtubules were redistributed from central spindles to asters. This might have an impact on the generation of stable microtubule-kinetochore attachments, which would then serve as cause for the observed abovementioned effects. Of course, further research would be required to prove this idea or to figure out other reasons responsible for the effects observed for Nocodazole.

\subsection{The role of EB1 binding for the functions of TRIO and its effector Rac1}

While the immunofluorescence images of TRIO clearly showed an EB1 dependent microtubule plus-end localization, the generated HCT116 clones with TRIO overexpression did rather show a strong overall localization. But what is the reason for this difference in the observed localization? TRIO is a multidomain protein and has many other interaction partners than EB1, thereby being located at different compartments of the cell. Due to its interaction with filamin A and Tara it is strongly associated with actin filaments, while its interaction with LAR also results in plasma membrane localization of TRIO (S. Schmidt \& Debant, 2014; van Rijssel \& van Buul, 2012). For immunofluorescence staining, however, methanol fixation was performed, as the anti-TRIO antibody that was used did not detect formaldehyde-fixed TRIO antigens. However, methanol destroys the actin cytoskeleton and also damages the cell membrane, thereby also ruling out the possibility to visualize associated proteins (Hoetelmans et al., 2001; Melak et al., 2017). For this reason, immunofluorescence images only show +TIP localization for TRIO, while in unfixed, live cell images of HCT116 clones TRIO was localized at several compartments. This observation is confirmed by van Haren et al. (2014), who found overexpressed mCherry-TRIO also largely diffused in the cytoplasm. Interestingly, work from van Haren et al. (2014) and unpublished work from our group showed that overexpression of TRIOs + TIP interaction partner Nav1 results in an increased recruitment of TRIO to microtubule plus ends that becomes visible in live cell images. This increase in + TIP localization was also seen when only overexpressing the GEF-D2 domain of TRIO, that harbors the SXIP domain responsible for EB1 binding (van Haren et al., 2014). As most other domains required for the interaction with other proteins are missing within this strongly truncated TRIO variant, it cannot localize to other compartments anymore. A part of the diffuse TRIO signal in these cells might be also due to overexpression of this protein, as an excess protein concentration is also associated with protein mis-localization (Huh et al., 2003).

GEF activity assays with HEK293T cells overexpressing the wildtype of TRIO, that has EB1 binding capacity, and the SXIP-mutated TRIO, that is deficient of EB1 binding, revealed that the GEF-D1 activity does not depend on EB1 binding. This finding is consistent with the observations from van Haren et al. (2014), who also observed no difference in GEF-D1 activity of wildtype TRIO 
and SXIP-mutated TRIO by performing fluorescence-based in vitro guanine nucleotide exchange assays measuring Mant-GTP loading onto GDP-preloaded RhoG. A HEK293T cell overexpression system was chosen, as GEF activity assays with HCT116 and SW620 cells endogenously expressing TRIO did not exhibit detectable active TRIO levels, but only detectable active TIAM1 levels. This is consistent with the findings of García-Mata et al. (2006), who figured out that TRIO is only able to bind to wildtype Rac1, but not to Rac1 G15A, that was used for binding active GEFs within the used GEF assays due to the higher stability of this mutant compared to wildtype Rac1. However, van Rijssel et al. (2012) successfully performed GEF activity assays for TRIO by increasing TRIO levels and/or activity via TRIO overexpression or TNF-a-treatment. Consequently, TRIO was overexpressed in this study to successfully carry out GEF activity assays. Interestingly, Berger (2016) was able to detect active TRIO from GEF activity assays with untransfected HCT116 and SW620 cells. She could even observe a decrease in the GEF activity of TRIO in mitotic SW620 cells upon Taxol or TRIO inhibitor treatment. However, in GEF assays carried out by Berger (2016), mitotic HCT116 cells showed a level of active TRIO similar to SW620 cells, therefore it would also be of interest, whether Taxol or a TRIO inhibitor treatment might also decrease TRIO activity in these cells.

Due to the finding in this study, that the GEF-D1 activity of TRIO does not depend on EB1 binding, the question was raised whether there are still differences in the activation of downstream effectors. Indeed, it was found a difference in Rac1 activation by the EB1 binding TRIO wildtype and the EB1 binding deficient TRIO mutant. Rac1 activity assays performed in this study revealed that the EB1 binding capacity of TRIO is essential for Rac1 activation in mitotic cells, but not in interphase cells. This is consistent with the work from other groups, showing that EB1 is involved in mitotic spindle assembly and positioning, chromosome alignment and chromosomal instability (Green et al., 2005; Rogers et al., 2002; Rogers et al., 2004; Tirnauer \& Bierer, 2000). Especially Rogers et al. (2002) found out, that siRNA-mediated knock down of EB1 results in a dramatic decrease of microtubule dynamics, that did not affect microtubule organization in interphase, but resulted in severely reduced astral microtubules, a malformed mitotic spindle and spindle mispositioning, followed by chromosome mis-segregation. Interestingly, EB1 depletion did only affect the frequency of catastrophe and rescue events, but not the growth or shrinkage rate. This is consistent with work from Berger (2016), who also found no difference in the microtubule growth rate of EB1 depleted and control SW620 cells. However, she detected a slight decrease in the amount of lagging chromosomes after partial EB1 depletion, suggesting rather a rescue of chromosome mis-segregation. This observation can be probably explained by findings of more recent studies, which figured out that EB1 is frequently overexpressed in colorectal cancer in 
correlation with a poor prognosis (Gemoll et al., 2017; Stypula-Cyrus et al., 2014; Sugihara et al., 2012). As SW620 cells are chromosomally unstable colorectal cancer cells, that are associated with a poor prognosis, these might inherit upregulated EB1 levels. Consequently, a partial depletion of EB1 would restore normal EB1 levels, resulting in the rescue observed from Berger (2016). Summed up, these findings demonstrate that alterations of the EB1 level in both directions might be involved in cancer development, thereby claiming that the EB1 level needs to be tightly balanced.

So far, most effects seen for EB1 downregulation were mainly linked to its interaction partners APC and RhoGEF2 (Green et al., 2005; Rogers et al., 2004). The present study now introduced Rac1 as downstream effector of EB1 binding dependent TRIO is mitosis, thereby generating a putative link between the described cancer-related overexpression of EB1 and TRIO-mediated hyperactivation of Rac1 in mitotic cells. Interestingly, not only EB1 is frequently up-regulated in colorectal cancer with poor prognosis, but also TRIO was found to be strongly overexpressed in cancers with karyotype heterogeneity (Sheltzer, 2013) and correlates with poor prognosis of patients with colorectal cancer (Sonoshita et al., 2015).

While EB1 dependent TRIO binding at microtubule plus-ends seems to be essential for Rac1 activation in mitosis, it is not required for TRIO-mediated Rac1 activation in interphase. This could explain, why there is an increase in cell migration, which is known to be an essential driver of cancer cell metastasis, observed for both TRIO variants. This migration-inducing effect of TRIO has also been reported by van Rijssel et al. (2012). Interestingly, the EB1 binding deficient mutant of TRIO even showed a stronger increase in cell migration than wildtype TRIO. This observation is consistent with the stronger localization of the TRIO mutant at lamellipodia when compared to wildtype TRIO. Moreover, this finding goes along with a study from van Haren et al. (2014), who observed stronger lamellipodia formation when TRIO localization at microtubule plus-ends was decreased via re-expression of a microtubule plus-end binding deficient mutant of TRIOs +TIP interaction partner Nav1.

Although there was a stronger increase in cell migration upon overexpression of the EB1 binding deficient TRIO mutant, the increase in Rac1 activation was still higher for wildtype TRIO than for the TRIO mutant. One possible explanation might be, that due to the differences in localization of these two TRIO variants, Rac1 is activated at different sites that not all contribute to cell migration. To be more precise, a part of TRIO-WT-GFP is probably located at microtubule plus-tip-bound EB1 and might thereby not be available for migration-related processes at lamellipodia. In contrast, TRIO-SRNN-GFP is not bound to EB1 at microtubule plus-tips and, thus, a larger amount of TRIO-SRNN-GFP might be available for inducing migration-driving processes at lamellipodia. 
In addition, it has also been reported that a high Rac1 activity alone is not always sufficient for an increase in cell migration and that this depends on the cell type and culturing conditions (A. J. Ridley, 2001). Moreover, other Rho GTPases, such as RhoA, RhoG and Cdc42, are involved in the dynamic and spatially tightly regulated cytoskeletal changes required for successful cell migration (Katoh et al., 2006; A. J. Ridley, 2001). Since TRIO is not only a GEF for Rac1, but also for RhoG and RhoA, activation of the latter Rho GTPases might also play an important role for the impact of TRIO on cell migration. Therefore, it would be of need to further analyze, how wildtype TRIO and the EB1 binding deficient mutant of TRIO affect RhoA and RhoG activity.

As overexpression systems usually do not represent the physiological condition, Rac1 assays with endogenously expressed TRIO have also been performed within this study. However, they did not reveal a change in Rac1 activity in mitotic SW620 cells upon treatment with low dose Taxol or inhibitors against TRIO or Rac1. This might be due to the fact that Rac1 activity decreases rapidly when a cell enters mitosis (Yoshizaki et al., 2003), which does not allow to detect further reduction. GEF assays performed in this study affirm a low mitotic Rac1 activity, as they show a lower amount of active TIAM1 in mitotic cells when compared to interphase cells and this was even lower for SW620 than for HCT116 cells. Moreover, TRIO levels were also lower in mitosis than in interphase. However, in Rac1 activity assays carried out by Berger (2016) there was even a visible decrease after treatment with low dose Taxol, TRIO inhibitor or Rac1 inhibitor. This result could also be seen in the present study when using phospho-PAK1/2 levels as readout for Rac1 activity, but only after TRIO or Rac1 inhibition and not upon Taxol treatment. Moreover, Rac1 assays performed in this study revealed a higher Rac1 activity in mitotic HCT116 cells than in mitotic SW620 cells. Unpublished data showed Rac1 levels of these two cell lines to be generally higher in interphase than in mitosis and they also showed a higher Rac1 activity for interphase HCT116 than for SW620 cells, which is consistent with work from Toledo et al. (2012). Surprisingly, Berger (2016) detected a higher mitotic Rac1 activity in SW620 than in HCT116 cells. These differences in the results make clear, that the Rac1 activity assay is quite error-prone when used for cells or cell cycle phases that intrinsically exhibit low active Rac1 levels. Consequently, this assay has to be further improved in order to generate meaningful results. In this context, quantification of the phospho-PAK1/2 level as readout for Rac1 activity might be a useful alternative. However, detection and quantification of the whole PAK1/2 level should be performed as an additional control.

However, even in case the mitotic Rac1 activity is higher in chromosomally stable HCT116 than in chromosomally unstable SW620 cells, this does not directly mean that there is no microtubule dynamic, EB1, and TRIO dependent activation of Rac1 existing in chromosomally unstable cells. 
Due to the fact, that only a small part of the whole pool of Rac1 might be involved in the given pathway, changes in the overall Rac1 activity only due to alterations in microtubule dynamics might not be easily detectable. This would mean, that there might be still a part of Rac1 being activated by TRIO that is located at microtubule plus-tip bound EB1, but it cannot be visualized under these conditions.

\subsection{The role of TRIO in the induction of whole and structural chromosomal instability}

As mentioned above, TRIO was found to be strongly upregulated in cancers with karyotype heterogeneity (Sheltzer, 2013) and correlates with poor prognosis of patients with colorectal cancer (Sonoshita et al., 2015). This is consistent with the finding in the present study, that chromosomally unstable SW620 cells have a higher TRIO level than chromosomally stable HCT116 cells. In this context, this study could show for the first time, that TRIO overexpression in originally chromosomally stable HCT116 cells results in whole as well as structural chromosome instability. Interestingly, the observed whole chromosome instability was almost completely dependent on the EB1 binding capacity of TRIO, as HCT116 cell clones overexpressing the EB1 binding deficient TRIO mutant only exhibited a very slight increase in karyotype heterogeneity. Moreover, the observed whole chromosome instability correlated with an increase in spindle misorientation and in the occurrence of lagging chromosomes, thereby suggesting TRIO to induce whole chromosome instability via an EB1 binding dependent involvement in spindle positioning. The finding, that the observed whole chromosome instability could be rescued by inhibition of components of the introduced EB1-TRIO-Rac1-Arp2/3 pathway confirms the role of TRIO and especially of its Rac1-activating GEF-D1 domain within this pathway as well as the role of this pathway in the induction of chromosomal instability. In contrast, the observed structural chromosome instability in form of dicentric chromosomes and acentric double minutes was observed in both the TRIO wildtype with EB1 binding capacity and the TRIO mutant with EB1 binding deficiency. However, the amount of structural chromosome aberrations was clearly higher in wildtype TRIO than in mutant TRIO overexpressing cells. The finding that cells overexpressing wildtype TRIO do not only show whole, but also structural chromosome instability is consistent with recent studies, who revealed a correlation between whole and structural chromosome instability (Sansregret et al., 2018). Crasta et al. (2012) demonstrated that a CIN-associated generation of lagging chromosomes results in the formation of micronuclei that are involved in the generation of DNA breaks and thus structural chromosome aberrations. Moreover, Burrell et al. (2013) found out that the generation of replication stress, which occurs due to the frequent loss of CIN-suppressor genes located on chromosome 18q, leads not only to DNA double strand breaks 
and thus structural chromosome aberrations, but also to whole chromosome mis-segregation. Interestingly, our own group further found replication stress to be associated with impaired mitotic microtubule dynamics, that was identified as a trigger for whole chromosomal instability (Böhly et al., 2019). Moreover, a correlation between W-CIN and S-CIN was reported to be multidirectional: oncogenic pathways can trigger S-CIN or W-CIN, with the latter one acting itself as trigger for S-CIN (Bakhoum \& Swanton, 2014). In turn, both S-CIN and W-CIN can induce oncogenic pathways due to loss of tumor suppressor genes or gain of oncogenes. Furthermore, aneuploidy in form of a W-CIN-driven gain of chromosomes also was shown to induce replication stress (Passerini et al., 2016). Taken together, the results from the abovementioned studies revealed a complex relationship between the induction of W-CIN and S-CIN (Figure 4.2).

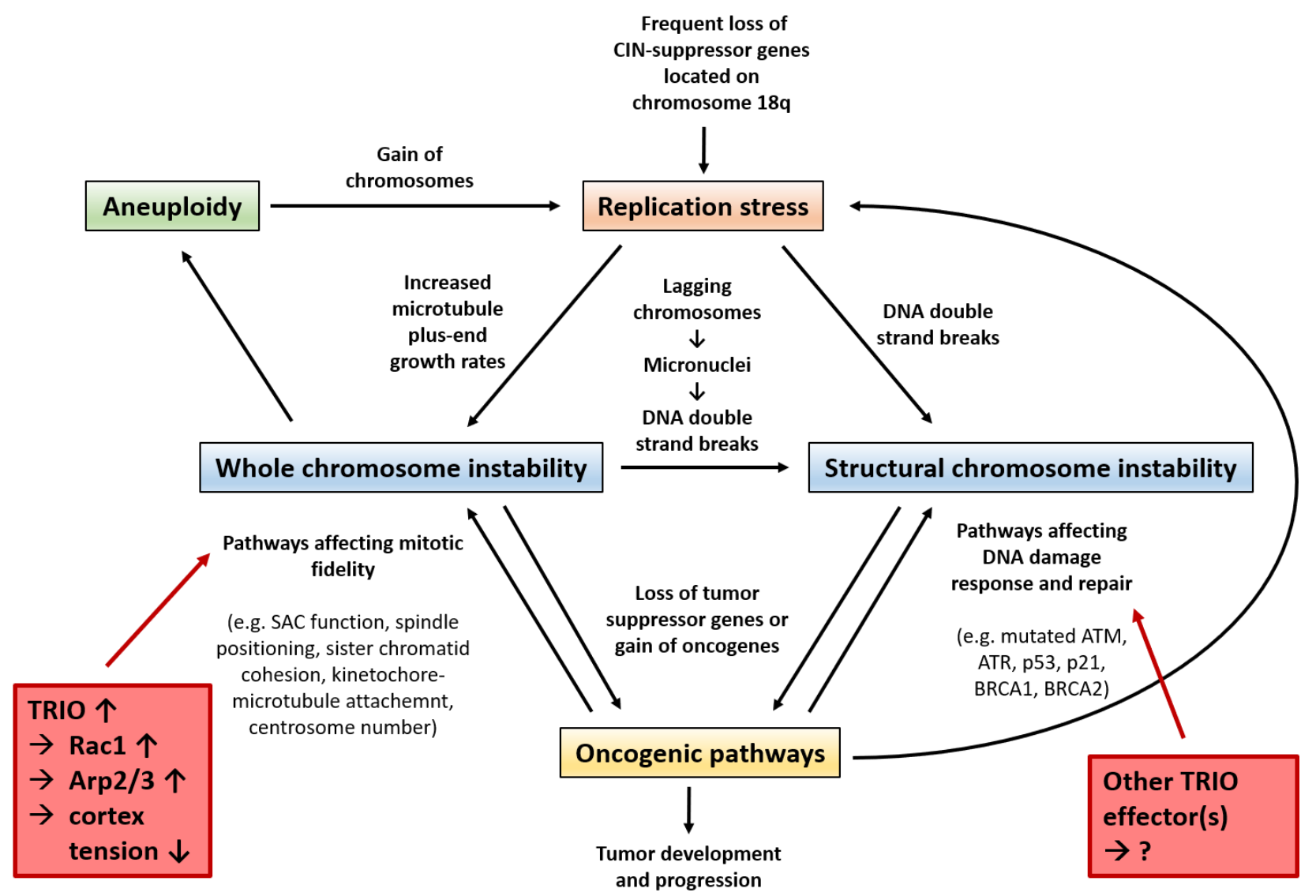

Figure 4.2: Correlations between the induction of W-CIN and S-CIN. W-CIN and S-CIN can both be induced by replication stress and/or oncogenic pathways, but they can also trigger oncogenic pathways themselves. S-CIN can be also induced by W-CIN. Moreover, W-CIN results in aneuploid cells, from which those with a gain of chromosomes trigger replication stress. TRIO induces W-CIN via binding to EB1, followed by GEF-D1-mediated activation of downstream Rac1, that in turn activates Arp2/3, thereby reducing cortex tension. This results in spindle mispositioning, which triggers chromosome mis-segregation and thus W-CIN. TRIO also induces structural chromosome instability, but via an EB1 binding and GEF-D1 independent hitherto unknown effector and mechanism. 
However, while inhibition of components of the EB1-TRIO-Rac1-Arp2/3 pathway in wildtype TRIO overexpressing cells resulted in a strong rescue of W-CIN, it only caused a partial rescue of S-CIN. This suggests that the observed S-CIN is not only caused via a correlation between the induction of W-CIN and S-CIN, but most likely also by another TRIO-mediated mechanism. Moreover, inhibition of components of the EB1-TRIO-Rac1-Arp2/3 pathway did not rescue S-CIN in cells with overexpression of the EB1 binding deficient mutant of TRIO, thereby further affirming the existence of another TRIO pathway involved in S-CIN development (Figure 4.2). As neither inhibition of the TRIO GEF-D1 domain nor the inhibition of Rac1 results in a rescue, this pathway does not only seem to be EB1 binding independent but also Rac1 independent. TRIO is a multidomain protein, which leaves many other interaction partners left to be the possible effector that is responsible for the induction of S-CIN. Interestingly, recent work from Osaki et al. (2016) showed that both an increase and a decrease of active RhoA impairs the induction of DNA damage repair, thereby causing increased levels of DNA damage. This finding leads to the suggestion, that RhoA might be the TRIO effector responsible for the induction of S-CIN. However, further research would need to be done to prove this or to figure out the actual sought-for TRIO effector.

The importance of a tightly regulated expression and activity has already been described for EB1, as a downregulation was shown to cause spindle mispositioning and chromosome missegregation, while an upregulation correlates with colorectal cancers that exhibit a poor prognosis. However, a tightly balanced activity is not only relevant for EB1, but it has also been shown to be important for TRIO. While the present study demonstrates that an overexpression of TRIO results in W-CIN and S-CIN, Liskovykh et al. (2019) showed similar effects upon siRNA-mediated depletion of TRIO, as they also observed an induction in the formation of lagging chromosomes and anaphase bridges.

\subsection{The EB1-TRIO-Rac1-Arp2/3 dependent pathway represents the missing link between microtubule dynamics and spindle positioning by modulating mitotic cortex tension}

Recent studies from our group showed that an increase in microtubule plus-end growth rates correlates with a transient spindle mis-positioning (Ertych et al., 2014; Schermuly, 2019). Further work from our group as well as the present study suggest that the introduced EB1-TRIO-Rac1Arp2/3 pathway represents the missing link. Although Mitsushima et al. (2010) and Kwon et al. (2015) described the existence of mitotic subcortical actin clouds and their importance for correct spindle positioning, these actin structures seem not to be the major downstream target of the EB1- 
TRIO-Rac1-Arp2/3 pathway but it is rather the actin cortex. Nevertheless, in this study the actin intensity of subcortical actin clouds always changed simultaneously with the intensity of the actin cortex. Interestingly, most results from actin intensity measurements are consistent with the results from AFM indentation experiments, as a downregulation of the EB1-TRIO-Rac1-Arp2/3 pathway in chromosomally unstable SW620 cells resulted in a decreased actin intensity together with an increased cortex tension. Upon downregulation of pathway components, mitotic cortex tension of SW620 cells could even be rescued to the level of chromosomally stable HCT116 cells. Downregulation of pathway components in mitotic HCT116 cells resulted in a slightly less pronounced decrease in the intensity of the actin cytoskeleton and also in an only slight further increase in cortex tension after Rac1 inhibition. These results demonstrate that chromosomally stable HCT116 cells but not chromosomally unstable SW620 cells generate a sufficient increase in cortex tension upon mitotic entry and that this tension is affected by the activity of the EB1TRIO-Rac1-Arp2/3 pathway rather in CIN cells than in non-CIN cells. Unpublished data confirmed that the measured differences in cortical tension are actually due to changes within the actomyosin cortex, as there was no change in plasma membrane tension. Cortex tension could also be reduced in both HCT116 and SW620 cells by inhibition of myosin II, that is one major driver for cortical contractility. However, myosin II inhibition also resulted in a decreased actin cortex intensity, thereby indicating that not every decrease in actin intensity results in an increase in cortex tension. In fact, it is not surprising that myosin II inhibition decreases actin intensity, as to fulfil its function as contractile force inducer myosin II acts as actin crosslinker and was also reported to support other actin crosslinkers (Laevsky \& Knecht, 2003; Luo \& Robinson, 2010). Similar as a reduction of Arp2/3 activity, also a reduction of myosin II activity results in decreased actin crosslinking and thus actin density. Recent work from Ennomani et al. (2016) demonstrated, that a tightly balanced level of actin crosslinking is required to increase cortex tension, as both to less and to strong crosslinking of actin filaments results in decreased tension. In conclusion, a hyperactivation of the introduced microtubule plus-end-EB1-TRIO-Rac1-Arp2/3 pathway probably leads to a too strong actin crosslinking and branching resulting in decreased cortical tension. This can be rescued by downregulation of the pathway as demonstrated for chromosomally unstable SW620 cells. Chromosomally stable HCT116 cells only showed a slight decrease in F-actin signal intensity and thus actin density upon inhibition of TRIO or Rac1, that was stronger upon Arp2/3 inhibition. This indicates that the introduced pathway seems to play a minor role for these cells. When considering the model that an optimal actin crosslinking is required for the generation of sufficient cortex tension and that HCT116 cells exhibit this optimal crosslinking, one would expect a decrease in cortex tension after TRIO, Rac1 or Arp2/3. However, Rac1-inhibited HCT116 cells 
even showed a slight increase in cortex tension, thereby suggesting that the small, Rac1 inhibitioninduced change within the crosslinking level of the actin cytoskeleton still contributed to the formation of an actin cortex architecture optimal for the generation cortical tension. As Arp2/3 inhibition caused a much stronger decrease in actin cortex density of HCT116 cells, it would be the aim of future experiments to reveal whether this Arp2/3 inhibition decreases actin crosslinking to a level that deviates from the optimum to an extent, that then results in reduced cortical tension. In addition to actin filament crosslinking, Chugh et al. (2017) demonstrated that the actin cortex thickness also plays an important role for the generation of mitotic cortex tension. Similar to actin crosslinking, an optimal actin cortex thickness is required for a sufficient increase in cortical tension, as both a decrease and an increase in cortex thickness have been shown to reduce cortical tension. Chugh et al. (2017) showed that especially the level and activity of actin filament length regulators, such as $\mathrm{mDia}$, capping protein and cofilin, are responsible for actin cortex thickness, but not actin crosslinking proteins like Arp2/3, $\alpha$-actinin or filamin A, and also not cortexmembrane crosslinking proteins, such as ERM proteins. This is consistent with actin cortex

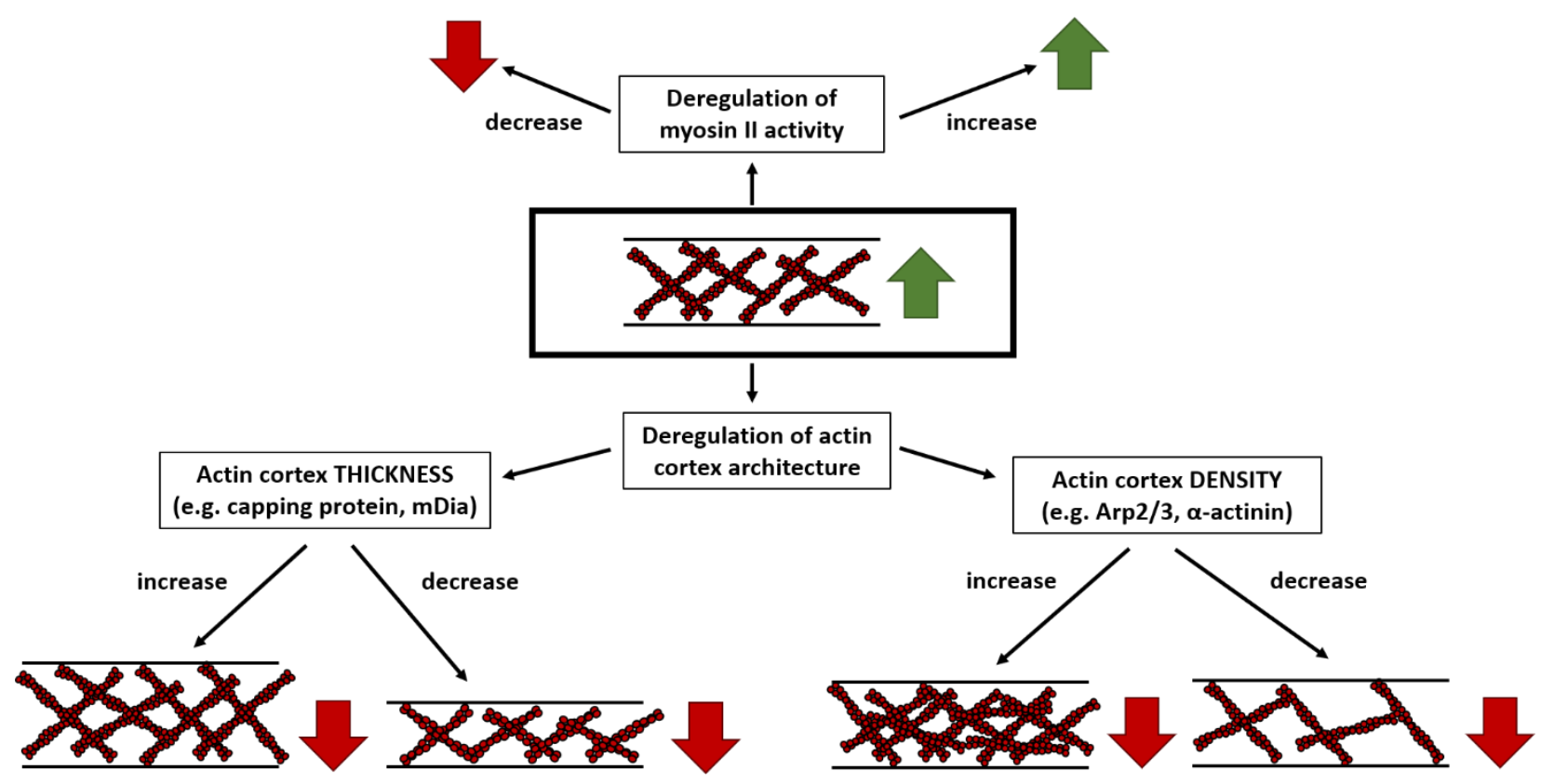

Figure 4.3: Model for the impact of actin cortex architecture and myosin II on cortical tension. Mitotic cell rounding depends on an increase in cortex tension (green arrow) that requires an optimal actin cortex thickness and density, as well as sufficient myosin II-mediated contractility. Both a deregulation of actin cortex thickness via actin filament length regulators, such as capping protein and mDia, and of actin cortex density via actin filament crosslinking proteins like Arp2/3 and $\alpha$-actinin results in a decreased cortical tension (red arrow). Moreover, a decreased myosin II activity also results in a reduced cortex tension, whereas an irregular increase in myosin II activity further increases cortical tension. Every deregulation, either inducing a decreased or an irregularly increased cortex tension, impairs mitotic spindle positioning. 
thickness measurements from the present study, as a downregulation of the EB1-TRIO-Rac1Arp2/3 pathway did not change cortex thickness. The same effect was reported by CartagenaRivera et al. (2016), who also measured no difference in cortex thickness, but an increase in cortical tension in Arp2/3-inhibited, nonadherent HFF cells.

Taken all results together, this gives rise to a model, in which an optimal actin cortex thickness, regulated by actin filament length regulators, and an optimal actin cortex density, regulated by actin filament crosslinkers, is required for the generation of sufficient mitotic cortex tension (Figure 4.3). Deviations from any optimum to any direction always results in a decreased cortical tension. Following this model, an increase in actin filament length would lead to an increase in actin cortex thickness and thus a reduced cortex tension. RhoA is a well-known activator of mDia formins, so that an increase in RhoA activation would be suggested to cause an increase in actin filament length via hyperactivated $\mathrm{mDia}$, whereas inhibition of formins would reduce cortex thickness. However, in the present study there was no increase in actin cortex thickness measurable after treatment with low concentrations of the RhoA activator calpeptin and no decrease upon treatment with low dose of the formin inhibitor SMIFH2. At first glance, these results argue against the introduced model, but in this context it is of special importance to note that in a study from Ramanathan et al. (2015) the 50-100 fold concentration of calpeptin was used to induce slight changes in actin cortex thickness. Moreover, they showed that about a 2-fold concentration of SMIFH2 was required to induce significant changes in cortex tension. Consequently, actin cortex thickness can be altered by deregulation of RhoA or formin activity when using a higher drug concentration. Another important factor that needs to be considered is that activating RhoA via calpeptin does not only trigger the activation of mDia but also of the RhoA effector ROCK and thereby myosin II. This in turn has been shown to cause increased myosin II activity at the mitotic cortex (Ramanathan et al., 2015). Therefore, a strong increase in RhoA activity does not only lead to an increase in actin cortex thickness, but also myosin II-mediated contractility. Interestingly, Ramanathan et al. (2015) showed that in sum this still leads to an increase in cortical tension, thereby suggesting a stronger impact of myosin II than of cortex thickness following increased RhoA activation. This raises the question, whether cortical tension also has to be within a specific range to ensure correct spindle positioning in mitotic cells. Interestingly, Chaigne et al. (2015) revealed that this is at least the case for mouse oocytes, as both a too high or too low cortex tension caused defects in spindle positioning. In accordance with this study, Schermuly (2019) detected a defect in mitotic spindle positioning in chromosomally stable HCT116 cells upon RhoA inhibition by $\mathrm{C} 3$ toxin as well as by overexpression of a constitutively active RhoA mutant, thereby demonstrating that not only a decreased but also an excessively increased cortex tension results 
in spindle mispositioning. However, Schermuly (2019) measured a rescue in spindle positioning in chromosomally unstable SW620 cells after RhoA inhibition, which is not in line with the even more decreased cortex tension that these cells would exhibit after RhoA inhibition and also not with the heavily damaged actin cortex that especially SW620 cells showed after C3 toxin treatment. This observation might be explained by the strongly impaired cell rounding after C3 toxin treatment in SW620 cells, which does not allow the mitotic spindle poles to position randomly. This explanation is supported by work from Lázaro-Diéguez et al. (2015), who found out that the positioning of the mitotic spindle is restricted in its $x-z$ position due to constraints that are given when the mitotic cell is too flat. This probably also accounts as explanation for the apparent rescue of spindle orientation in SW620 cells, that have been treated with the actin-depolymerizing drug Latrunculin A, or the myosin II inhibitor blebbistatin, detected by Schermuly (2019). These treatments resulted in a damaged actin structure and/or a strong decrease in actin cortex tension, thereby probably impairing mitotic cell rounding and thus constraining the $x-z$ position of the spindle. Moreover, the fact that Latrunculin A ( $\mathrm{IC}_{50}=0.75 \mu \mathrm{M}$ and $0.33 \mu \mathrm{M}$ for MKN45 and NUGC4 cells, $72 \mathrm{~h}$ ) and blebbistatin ( $\mathrm{IC}_{50}=28.5 \mu \mathrm{M}$ for hBMSC cells, $48 \mathrm{~h}$ ) are known to induce cell death (Konishi et al., 2009; Paulamäki, 2017) and that C3 toxin has an antiproliferative effect (Elsner et al., 2017) rather excludes these drugs from causing an actual rescue.

The results from F-actin signal intensity and AFM measurements indicated that the actin cortex organization is somehow altered by the EB1-TRIO-Rac1-Arp2/3 pathway. Interestingly, STED experiments actually revealed a changed morphology of the actin cortex upon Rac1 or Arp2/3 inhibition, but not for low dose Taxol treatment in SW620 cells. However, even STED microscopy could still not visualize subtle changes, such as the pore size within the actin meshwork, that was reported to represent actin cortex density (Kronlage et al., 2015). In order to make these changes visible, scanning electron microscopy (SEM) (Bovellan et al., 2014; Chugh et al., 2017; Fritzsche et al., 2017), contact-mode AFM (Kronlage et al., 2015; Xia et al., 2019) or single molecule localization based super-resolution microscopy (SMLM) methods, such as STORM (Xia et al., 2019), have proved successful. Interestingly, SEM images from Fritzsche et al. (2017) as well as STORM images from Xia et al. (2019) demonstrated a larger pore size within the actin meshwork after Arp2/3 inhibition, which confirms the decreased actin density determined by F-actin signal intensity measurements in the present study. Therefore, it would be of utmost interest to use these super-resolution microscopy methods to investigate whether these changes in pore size of the actin meshwork are also induced via downregulation of components upstream of Arp2/3 within the introduced microtubule plus-end-EB1-TRIO-Rac1-Arp2/3 pathway. 


\section{References}

Abe, Y., Miura, T., Yoshida, M. A., Ujiie, R., Kurosu, Y., Kato, N., Katafuchi, A., Tsuyama, N., Ohba, T., Inamasu, T., Shishido, F., Noji, H., Ogawa, K., Yokouchi, H., Kanazawa, K., Ishida, T., Muto, S., Ohsugi, J., Suzuki, H., Ishikawa, T., Kamiya, K., \& Sakai, A. (2015). Increase in dicentric chromosome formation after a single CT scan in adults. Scientific Reports, 5, 13882. https://doi.org/10.1038/srep13882

Agircan, F. G., Schiebel, E., \& Mardin, B. R. (2014). Separate to operate: Control of centrosome positioning and separation. Philosophical Transactions of the Royal Society of London. Series B, Biological Sciences, 369(1650). https://doi.org/10.1098/rstb.2013.0461

Akhmanova, A., \& Steinmetz, M. O. (2008). Tracking the ends: A dynamic protein network controls the fate of microtubule tips. Nature Reviews. Molecular Cell Biology, 9(4), 309-322. https://doi.org/10.1038/nrm2369

Al-Bassam, J., \& Chang, F. (2011). Regulation of microtubule dynamics by TOG-domain proteins XMAP215/Dis1 and CLASP. Trends in Cell Biology, 21(10), 604-614. https://doi.org/10.1016/j.tcb.2011.06.007

Alberts, B., Johnson, A., Lewis, J., Morgan, D., Raff, M., Roberts, K., Walter, P., Wilson, J., \& Hunt, T. (2015). Molecular biology of the cell (Sixth edition). Garland Science Taylor and Francis Group.

Amano, M., Ito, M., Kimura, K., Fukata, Y., Chihara, K., Nakano, T., Matsuura, Y., \& Kaibuchi, K. (1996). Phosphorylation and activation of myosin by Rho-associated kinase (Rho-kinase). The Journal of Biological Chemistry, 271(34), 20246-20249. https://doi.org/10.1074/jbc.271.34.20246

Arellano, M., \& Moreno, S. (1997). Regulation of CDK/cyclin complexes during the cell cycle. The International Journal of Biochemistry \& Cell Biology, 29(4), 559-573. https://doi.org/10.1016/S1357-2725(96)00178-1

Bach, D.-H., Zhang, W., \& Sood, A. K. (2019). Chromosomal Instability in Tumor Initiation and Development. Cancer Research, 79(16), 3995-4002. https://doi.org/10.1158/00085472.CAN-18-3235

Bakhoum, S. F., \& Swanton, C. (2014). Chromosomal instability, aneuploidy, and cancer. Frontiers in Oncology, 4, 161. https://doi.org/10.3389/fonc.2014.00161 
Bao, Y., Liu, J., You, J., Wu, D., Yu, Y., Liu, C., Wang, L., Wang, F., Xu, L., Wang, L., Wang, N., Tian, X., Wang, F., Liang, H., Gao, Y., Cui, X., Ji, G., Bai, J., Yu, J., Meng, X., Jin, Y., Sun, W., Guan, X., Zhang, C., \& Fu, S. (2016). Met promotes the formation of double minute chromosomes induced by Sei-1 in NIH-3T3 murine fibroblasts. Oncotarget, 7(35), 5666456675. https://doi.org/10.18632/oncotarget.10994

Barber, T. D., McManus, K., Yuen, K. W. Y., Reis, M., Parmigiani, G., Shen, D., Barrett, I., Nouhi, Y., Spencer, F., Markowitz, S., Velculescu, V. E., Kinzler, K. W., Vogelstein, B., Lengauer, C., \& Hieter, P. (2008). Chromatid cohesion defects may underlie chromosome instability in human colorectal cancers. Proceedings of the National Academy of Sciences of the United States of America, 105(9), 3443-3448. https://doi.org/10.1073/pnas.0712384105

Barbero, J. L. (2011). Sister chromatid cohesion control and aneuploidy. Cytogenetic and Genome Research, 133(2-4), 223-233. https://doi.org/10.1159/000323507

Bartolini, F., Moseley, J. B., Schmoranzer, J., Cassimeris, L., Goode, B. L., \& Gundersen, G. G. (2008). The formin mDia2 stabilizes microtubules independently of its actin nucleation activity. The Journal of Cell Biology, 181(3), 523-536. https://doi.org/10.1083/jcb.200709029

Bellanger, J. M., Astier, C., Sardet, C., Ohta, Y., Stossel, T. P., \& Debant, A. (2000). The Rac1and RhoG-specific GEF domain of Trio targets filamin to remodel cytoskeletal actin. Nature Cell Biology, 2(12), 888-892. https://doi.org/10.1038/35046533

Berger, K. (2016). A Role For Microtubule Dynamics For The Induction Of Chromosomal Instability And Cell Migration And Invasion In Human Cancer Cells.

Biro, M., Munoz, M. A., \& Weninger, W. (2014). Targeting Rho-GTPases in immune cell migration and inflammation. British Journal of Pharmacology, 171(24), 5491-5506. https://doi.org/10.1111/bph.12658

Blangy, A., Vignal, E., Schmidt, S., Debant, A., Gauthier-Rouvière, C., \& Fort, P. (2000). Triogef1 controls Rac- and Cdc42-dependent cell structures through the direct activation of rhoG. Journal of Cell Science, 113 (Pt 4), 729-739.

Böhly, N., Kistner, M., \& Bastians, H. (2019). Mild replication stress causes aneuploidy by deregulating microtubule dynamics in mitosis. Cell Cycle, 18(20), 2770-2783. https://doi.org/10.1080/15384101.2019.1658477 
Bouquier, N., Vignal, E., Charrasse, S., Weill, M., Schmidt, S., Léonetti, J.-P., Blangy, A., \& Fort, P. (2009). A cell active chemical GEF inhibitor selectively targets the Trio/RhoG/Rac1 signaling pathway. Chemistry \& Biology, 16(6), 657-666.

https://doi.org/10.1016/j.chembiol.2009.04.012

Bovellan, M., Romeo, Y., Biro, M., Boden, A., Chugh, P., Yonis, A., Vaghela, M., Fritzsche, M., Moulding, D., Thorogate, R., Jégou, A., Thrasher, A. J., Romet-Lemonne, G., Roux, P. P., Paluch, E. K., \& Charras, G. (2014). Cellular control of cortical actin nucleation. Current Biology: CB, 24(14), 1628-1635. https://doi.org/10.1016/j.cub.2014.05.069

Bowne-Anderson, H., Hibbel, A., \& Howard, J. (2015). Regulation of Microtubule Growth and Catastrophe: Unifying Theory and Experiment. Trends in Cell Biology, 25(12), 769-779. https://doi.org/10.1016/j.tcb.2015.08.009

Braun, A., Dang, K., Buslig, F., Baird, M. A., Davidson, M. W., Waterman, C. M., \& Myers, K. A. (2014). Rac1 and Aurora A regulate MCAK to polarize microtubule growth in migrating endothelial cells. The Journal of Cell Biology, 206(1), 97-112.

https://doi.org/10.1083/jcb.201401063

Breitsprecher, D., \& Goode, B. L. (2013). Formins at a glance. Journal of Cell Science, 126(1), 1-7. https://doi.org/10.1242/jcs. 107250

Brouhard, G. J., Stear, J. H., Noetzel, T. L., Al-Bassam, J., Kinoshita, K., Harrison, S. C., Howard, J., \& Hyman, A. A. (2008). Xmap215 is a processive microtubule polymerase. Cell, 132(1), 79-88. https://doi.org/10.1016/j.cell.2007.11.043

Burrell, R. A., McClelland, S. E., Endesfelder, D., Groth, P., Weller, M.-C., Shaikh, N., Domingo, E., Kanu, N., Dewhurst, S. M., Gronroos, E., Chew, S. K., Rowan, A. J., Schenk, A., Sheffer, M., Howell, M., Kschischo, M., Behrens, A., Helleday, T., Bartek, J., Tomlinson, I. P., \& Swanton, C. (2013). Replication stress links structural and numerical cancer chromosomal instability. Nature, 494(7438), 492-496. https://doi.org/10.1038/nature11935

Cadart, C., Zlotek-Zlotkiewicz, E., Le Berre, M., Piel, M., \& Matthews, H. K. (2014). Exploring the Function of Cell Shape and Size during Mitosis. Developmental Cell, 29(2), 159-169. https://doi.org/10.1016/j.devcel.2014.04.009

Carminati, M., Gallini, S., Pirovano, L., Alfieri, A., Bisi, S., \& Mapelli, M. (2016). Concomitant binding of Afadin to LGN and F-actin directs planar spindle orientation. Nature Structural \& Molecular Biology, 23(2), 155-163. https://doi.org/10.1038/nsmb.3152 
Cartagena-Rivera, A. X., Logue, J. S., Waterman, C. M., \& Chadwick, R. S. (2016). Actomyosin Cortical Mechanical Properties in Nonadherent Cells Determined by Atomic Force Microscopy. Biophysical Journal, 110(11), 2528-2539.

https://doi.org/10.1016/j.bpj.2016.04.034

Chaigne, A., Campillo, C., Gov, N. S., Voituriez, R., Sykes, C., Verlhac, M. H., \& Terret, M. E. (2015). A narrow window of cortical tension guides asymmetric spindle positioning in the mouse oocyte. Nature Communications, 6(1), 1-10. https://doi.org/10.1038/ncomms7027

Chatterjee, N., \& Walker, G. C. (2017). Mechanisms of DNA damage, repair, and mutagenesis. Environmental and Molecular Mutagenesis, 58(5), 235-263.

https://doi.org/10.1002/em.22087

Cheeseman, I. M., \& Desai, A. (2008). Molecular architecture of the kinetochore-microtubule interface. Nature Reviews. Molecular Cell Biology, 9(1), 33-46.

https://doi.org/10.1038/nrm2310

Chen, P., O’Neal, J. F., Ebelt, N. D., Cantrell, M. A., Mitra, S., Nasrazadani, A., Vandenbroek, T. L., Heasley, L. E., \& van den Berg, C. L. (2010). Jnk2 effects on tumor development, genetic instability and replicative stress in an oncogene-driven mouse mammary tumor model. PloS One, 5(5), e10443.

https://doi.org/10.1371/journal.pone.0010443

Chesarone, M. A., DuPage, A. G., \& Goode, B. L. (2010). Unleashing formins to remodel the actin and microtubule cytoskeletons. Nature Reviews. Molecular Cell Biology, 11(1), 62-74. https://doi.org/10.1038/nrm2816

Chugh, P., Clark, A. G., Smith, M. B., Cassani, D. A. D., Dierkes, K., Ragab, A., Roux, P. P., Charras, G., Salbreux, G., \& Paluch, E. K. (2017). Actin cortex architecture regulates cell surface tension. Nature Cell Biology, 19(6), 689-697. https://doi.org/10.1038/ncb3525

Cimini, D., Wan, X., Hirel, C. B., \& Salmon, E. D. (2006). Aurora Kinase Promotes Turnover of Kinetochore Microtubules to Reduce Chromosome Segregation Errors. Current Biology, 16(17), 1711-1718. https://doi.org/10.1016/j.cub.2006.07.022

Cisyk, A. L., Penner-Goeke, S., Lichtensztejn, Z., Nugent, Z., Wightman, R. H., Singh, H., \& McManus, K. J. (2015). Characterizing the Prevalence of Chromosome Instability in Interval Colorectal Cancer. Neoplasia, 17(3), 306-316. https://doi.org/10.1016/j.neo.2015.02.001 
Clurman, B. E., \& Roberts, J. M. (1995). Cell cycle and cancer. Journal of the National Cancer Institute, 87(20), 1499-1501. https://doi.org/10.1093/jnci/87.20.1499

Collins, K., Jacks, T., \& Pavletich, N. P. (1997). The cell cycle and cancer. Proceedings of the National Academy of Sciences of the United States of America, 94(7), 2776-2778. https://doi.org/10.1073/pnas.94.7.2776

Cortez, D. (2015). Preventing replication fork collapse to maintain genome integrity. DNA Repair, 32, 149-157. https://doi.org/10.1016/j.dnarep.2015.04.026

Courtemanche, N. (2018). Mechanisms of formin-mediated actin assembly and dynamics. Biophysical Reviews, 10(6), 1553-1569. https://doi.org/10.1007/s12551-018-0468-6

Crasta, K., Ganem, N. J., Dagher, R., Lantermann, A. B., Ivanova, E. V., Pan, Y., Nezi, L., Protopopov, A., Chowdhury, D., \& Pellman, D. (2012). Dna breaks and chromosome pulverization from errors in mitosis. Nature, 482(7383), 53-58. https://doi.org/10.1038/nature10802

Cross, S. E., Jin, Y.-S., Rao, J., \& Gimzewski, J. K. (2007). Nanomechanical analysis of cells from cancer patients. Nature Nanotechnology, 2(12), 780-783. https://doi.org/10.1038/nnano.2007.388

Cuddihy, A. R., \& O'Connell, M. J. (2003). Cell-cycle responses to DNA damage in G2. In K. W. Jeon (Ed.), International Review of Cytology: v. 222. International review of cytology: A survey of cell biology (Vol. 222, pp. 99-140). Academic Press. https://doi.org/10.1016/S00747696(02)22013-6

Daub, H., Gevaert, K., Vandekerckhove, J., Sobel, A., \& Hall, A. (2001). Rac/cdc42 and p65PAK regulate the microtubule-destabilizing protein stathmin through phosphorylation at serine 16 . The Journal of Biological Chemistry, 276(3), 1677-1680. https://doi.org/10.1074/jbc.C000635200

Davidson, A. J., \& Wood, W. (2016). Unravelling the Actin Cytoskeleton: A New Competitive Edge? Trends in Cell Biology, 26(8), 569-576. https://doi.org/10.1016/j.tcb.2016.04.001

Debant, A., Serra-Pagès, C., Seipel, K., O’Brien, S., Tang, M., Park, S. H., \& Streuli, M. (1996). The multidomain protein Trio binds the LAR transmembrane tyrosine phosphatase, contains a protein kinase domain, and has separate rac-specific and rho-specific guanine nucleotide exchange factor domains. Proceedings of the National Academy of Sciences of the United States of America, 93(11), 5466-5471. https://doi.org/10.1073/pnas.93.11.5466 
Degasperi, A., Birtwistle, M. R., Volinsky, N., Rauch, J., Kolch, W., \& Kholodenko, B. N. (2014). Evaluating strategies to normalise biological replicates of Western blot data. PloS One, 9(1), e87293. https://doi.org/10.1371/journal.pone.0087293

Den Haese, G. J., Walworth, N., Carr, A. M., \& Gould, K. L. (1995). The Wee1 protein kinase regulates T14 phosphorylation of fission yeast Cdc2. Molecular Biology of the Cell, 6(4), 371385.

Derivery, E., \& Gautreau, A. (2010). Generation of branched actin networks: Assembly and regulation of the N-WASP and WAVE molecular machines. BioEssays: News and Reviews in Molecular, Cellular and Developmental Biology, 32(2), 119-131.

https://doi.org/10.1002/bies.200900123

Derry, W. B., Wilson, L., \& Jordan, M. A. (1998). Low potency of taxol at microtubule minus ends: Implications for its antimitotic and therapeutic mechanism. Cancer Research, 58(6), 1177-1184.

Deshpande, A., Sicinski, P., \& Hinds, P. W. (2005). Cyclins and cdks in development and cancer: A perspective. Oncogene, 24(17), 2909-2915. https://doi.org/10.1038/sj.onc.1208618

Di Pietro, F., Echard, A., \& Morin, X. (2016). Regulation of mitotic spindle orientation: An integrated view. EMBO Reports, 17(8), 1106-1130. https://doi.org/10.15252/embr.201642292

Dunican, D. S., McWilliam, P., Tighe, O., Parle-McDermott, A., \& Croke, D. T. (2002). Gene expression differences between the microsatellite instability (MIN) and chromosomal instability (CIN) phenotypes in colorectal cancer revealed by high-density cDNA array hybridization. Oncogene, 21(20), 3253-3257. https://doi.org/10.1038/sj.onc.1205431

Ellenbroek, S. I. J., \& Collard, J. G. (2007). Rho GTPases: Functions and association with cancer. Clinical \& Experimental Metastasis, 24(8), 657-672. https://doi.org/10.1007/s10585007-9119-1

Elsner, L. von, Hagemann, S., Just, I., \& Rohrbeck, A. (2017). Anti-proliferative Effect of C3 Exoenzyme in Fibroblasts is Mediated by c-Jun Phosphorylation. Journal of Molecular Signaling, 12, 1. https://doi.org/10.5334/1750-2187-12-1

Ennomani, H., Letort, G., Guérin, C., Martiel, J.-L., Cao, W., Nédélec, F., La Cruz, E. M. de, Théry, M., \& Blanchoin, L. (2016). Architecture and Connectivity Govern Actin Network Contractility. Current Biology: CB, 26(5), 616-626. https://doi.org/10.1016/j.cub.2015.12.069 
Ertych, N., Stolz, A., Stenzinger, A., Weichert, W., Kaulfuß, S., Burfeind, P., Aigner, A., Wordeman, L., \& Bastians, H. (2014). Increased microtubule assembly rates influence chromosomal instability in colorectal cancer cells. Nature Cell Biology, 16(8), 779-791. https://doi.org/10.1038/ncb2994

Etienne-Manneville, S. (2013). Microtubules in cell migration. Annual Review of Cell and Developmental Biology, 29, 471-499. https://doi.org/10.1146/annurev-cellbio-101011-155711

Feher, J. J. (2017). Quantitative human physiology: An introduction (Second edition). Academic Press series in biomedical engineering. Elsevier/AP.

Fehon, R. G., McClatchey, A. I., \& Bretscher, A. (2010). Organizing the cell cortex: The role of ERM proteins. Nature Reviews. Molecular Cell Biology, 11(4), 276-287. https://doi.org/10.1038/nrm2866

Fife, C. M., McCarroll, J. A., \& Kavallaris, M. (2014). Movers and shakers: Cell cytoskeleton in cancer metastasis. British Journal of Pharmacology, 171(24), 5507-5523. https://doi.org/10.1111/bph.12704

Fink, J., Carpi, N., Betz, T., Bétard, A., Chebah, M., Azioune, A., Bornens, M., Sykes, C., Fetler, L., Cuvelier, D., \& Piel, M. (2011). External forces control mitotic spindle positioning. Nature Cell Biology, 13(7), 771-778. https://doi.org/10.1038/ncb2269

Firat-Karalar, E. N., \& Welch, M. D. (2011). New mechanisms and functions of actin nucleation. Current Opinion in Cell Biology, 23(1), 4-13. https://doi.org/10.1016/j.ceb.2010.10.007

Fischer-Friedrich, E., Hyman, A. A., Jülicher, F., Müller, D. J., \& Helenius, J. (2014). Quantification of surface tension and internal pressure generated by single mitotic cells. Scientific Reports, 4, 6213. https://doi.org/10.1038/srep06213

Fletcher, D. A., \& Mullins, R. D. (2010). Cell mechanics and the cytoskeleton. Nature, 463(7280), 485-492. https://doi.org/10.1038/nature08908

Forbes, D. J., Travesa, A., Nord, M. S., \& Bernis, C. (2015). Nuclear transport factors: Global regulation of mitosis. Current Opinion in Cell Biology, 35, 78-90.

https://doi.org/10.1016/j.ceb.2015.04.012

Friedl, P., \& Wolf, K. (2009). Proteolytic interstitial cell migration: a five-step process. Cancer and Metastasis Reviews, 28(1), 129-135. https://doi.org/10.1007/s10555-008-9174-3 
Fritzsche, M., Li, D., Colin-York, H., Chang, V. T., Moeendarbary, E., Felce, J. H., Sezgin, E., Charras, G., Betzig, E., \& Eggeling, C. (2017). Self-organizing actin patterns shape membrane architecture but not cell mechanics. Nature Communications, 8, 14347. https://doi.org/10.1038/ncomms14347

Funabiki, H. (2019). Correcting aberrant kinetochore microtubule attachments: a hidden regulation of Aurora B on microtubules. Current Opinion in Cell Biology, 58, 34-41. https://doi.org/10.1016/j.ceb.2018.12.007

Gaillard, H., García-Muse, T., \& Aguilera, A. (2015). Replication stress and cancer. Nature Reviews Cancer, 15(5), 276-289. https://doi.org/10.1038/nrc3916

Ganem, N. J., Godinho, S. A., \& Pellman, D. (2009). A mechanism linking extra centrosomes to chromosomal instability. Nature, 460(7252), 278-282. https://doi.org/10.1038/nature08136

García-Mata, R., Wennerberg, K., Arthur, W. T., Noren, N. K., Ellerbroek, S. M., \& Burridge, K. (2006). Analysis of Activated GAPs and GEFs in Cell Lysates. In A. Hall, C. J. Der, \& W. E. Balch (Eds.), Methods in Enzymology: v. 406. Regulators and effectors of small GTPases: Rho family (Vol. 406, pp. 425-437). Elsevier. https://doi.org/10.1016/S0076-6879(06)06031-9

Garcin, C., \& Straube, A. (2019). Microtubules in cell migration. Essays in Biochemistry, 63(5), 509-520. https://doi.org/10.1042/EBC20190016

Gemoll, T., Kollbeck, S. L., Karstens, K. F., Hò, G. G., Hartwig, S., Strohkamp, S., Schillo, K., Thorns, C., Oberländer, M., Kalies, K., Lehr, S., \& Habermann, J. K. (2017). Eb1 protein alteration characterizes sporadic but not ulcerative colitis associated colorectal cancer. Oncotarget, 8(33), 54939-54950. https://doi.org/10.18632/oncotarget.18978

Glotzer, M. (2005). The molecular requirements for cytokinesis. Science, 307(5716), 1735-1739. https://doi.org/10.1126/science.1096896

Godek, K. M., Kabeche, L., \& Compton, D. A. (2015). Regulation of kinetochore-microtubule attachments through homeostatic control during mitosis. Nature Reviews. Molecular Cell Biology, 16(1), 57-64. https://doi.org/10.1038/nrm3916

Goley, E. D., \& Welch, M. D. (2006). The ARP2/3 complex: An actin nucleator comes of age. Nature Reviews. Molecular Cell Biology, 7(10), 713-726. https://doi.org/10.1038/nrm2026

Goode, B. L., \& Eck, M. J. (2007). Mechanism and function of formins in the control of actin assembly. Annual Review of Biochemistry, 76, 593-627.

https://doi.org/10.1146/annurev.biochem.75.103004.142647 
Goshima, G., \& Scholey, J. M. (2010). Control of mitotic spindle length. Annual Review of Cell and Developmental Biology, 26, 21-57. https://doi.org/10.1146/annurev-cellbio-100109104006

Green, R. A., Wollman, R., \& Kaplan, K. B. (2005). Apc and EB1 function together in mitosis to regulate spindle dynamics and chromosome alignment. Molecular Biology of the Cell, 16(10), 4609-4622. https://doi.org/10.1091/mbc.e05-03-0259

Gregan, J., Polakova, S., Zhang, L., Tolić-Nørrelykke, I. M., \& Cimini, D. (2011). Merotelic kinetochore attachment: Causes and effects. Trends in Cell Biology, 21(6), 374-381. https://doi.org/10.1016/j.tcb.2011.01.003

Guo, Y., Kim, C., \& Mao, Y. (2013). New insights into the mechanism for chromosome alignment in metaphase. International Review of Cell and Molecular Biology, 303, 237-262. https://doi.org/10.1016/B978-0-12-407697-6.00006-4

Haga, R. B., \& Ridley, A. J. (2016). Rho GTPases: Regulation and roles in cancer cell biology. Small GTPases, 7(4), 207-221. https://doi.org/10.1080/21541248.2016.1232583

Hall, A. (1998). Rho GTPases and the actin cytoskeleton. Science, 279(5350), 509-514. https://doi.org/10.1126/science.279.5350.509

Hanahan, D., \& Weinberg, R. A. (2000). The Hallmarks of Cancer. Cell, 100(1), 57-70. https://doi.org/10.1016/S0092-8674(00)81683-9

Harbour, J. W., \& Dean, D. C. (2000). The Rb/E2F pathway: Expanding roles and emerging paradigms. Genes \& Development, 14(19), 2393-2409. https://doi.org/10.1101/gad.813200

Heald, R., \& Khodjakov, A. (2015). Thirty years of search and capture: The complex simplicity of mitotic spindle assembly. The Journal of Cell Biology, 211(6), 1103-1111.

https://doi.org/10.1083/jcb.201510015

Heald, R., Tournebize, R., Habermann, A., Karsenti, E., \& Hyman, A. (1997). Spindle Assembly in Xenopus Egg Extracts: Respective Roles of Centrosomes and Microtubule SelfOrganization. The Journal of Cell Biology, 138(3), 615-628.

Heng, H. H., Bremer, S. W., Stevens, J. B., Horne, S. D., Liu, G., Abdallah, B. Y., Ye, K. J., \& Ye, C. J. (2013). Chromosomal instability (CIN): What it is and why it is crucial to cancer evolution. Cancer Metastasis Reviews, 32(3-4), 325-340. https://doi.org/10.1007/s10555013-9427-7 
Henty-Ridilla, J. L., Rankova, A., Eskin, Julian A., Kenny, Katelyn, \& Goode, B. L. (2016). Accelerated actin filament polymerization from microtubule plus-ends. Science, 352(6288), 1004-1009. https://doi.org/10.1126/science.aaf1709

Higgs, H. N., \& Pollard, T. D. (1999). Regulation of actin polymerization by Arp2/3 complex and WASp/Scar proteins. The Journal of Biological Chemistry, 274(46), 32531-32534. https://doi.org/10.1074/jbc.274.46.32531

Higgs, H. N., \& Peterson, K. J. (2005). Phylogenetic analysis of the formin homology 2 domain. Molecular Biology of the Cell, 16(1), 1-13. https://doi.org/10.1091/mbc.e04-07-0565

Hoetelmans, R. W., Prins, F. A., Cornelese-ten Velde, I., van der Meer, J., van de Velde, C. J., \& van Dierendonck, J. H. (2001). Effects of acetone, methanol, or paraformaldehyde on cellular structure, visualized by reflection contrast microscopy and transmission and scanning electron microscopy. Applied Immunohistochemistry \& Molecular Morphology: AIMM, 9(4), 346-351. https://doi.org/10.1097/00129039-200112000-00010

Hohmann, T., \& Dehghani, F. (2019). The Cytoskeleton-A Complex Interacting Meshwork. Cells, 8(4). https://doi.org/10.3390/cells8040362

Holland, A. J., \& Cleveland, D. W. (2012). Losing balance: The origin and impact of aneuploidy in cancer. EMBO Reports, 13(6), 501-514. https://doi.org/10.1038/embor.2012.55

Honnappa, S., Gouveia, S. M., Weisbrich, A., Damberger, F. F., Bhavesh, N. S., Jawhari, H., Grigoriev, I., van Rijssel, F. J. A., Buey, R. M., Lawera, A., Jelesarov, I., Winkler, F. K., Wüthrich, K., Akhmanova, A., \& Steinmetz, M. O. (2009). An EB1-binding motif acts as a microtubule tip localization signal. Cell, 138(2), 366-376. https://doi.org/10.1016/j.cell.2009.04.065

Hou, C., Zhuang, Z., Deng, X., Xu, Y., Zhang, P., \& Zhu, L. (2018). Knockdown of Trio by CRISPR/Cas9 suppresses migration and invasion of cervical cancer cells. Oncology Reports, 39(2), 795-801. https://doi.org/10.3892/or.2017.6117

Huang, H., Lampson, M., Efimov, A., \& Yen, T. J. (2018). Chromosome instability in tumor cells due to defects in Aurora B mediated error correction at kinetochores. Cell Cycle, 17(23), 2622-2636. https://doi.org/10.1080/15384101.2018.1553340

Huh, W.-K., Falvo, J. V., Gerke, L. C., Carroll, A. S., Howson, R. W., Weissman, J. S., \& O'Shea, E. K. (2003). Global analysis of protein localization in budding yeast. Nature, 425(6959), 686-691. https://doi.org/10.1038/nature02026 
Hunter, A. W., Caplow, M., Coy, D. L., Hancock, W. O., Diez, S., Wordeman, L., \& Howard, J. (2003). The Kinesin-Related Protein MCAK Is a Microtubule Depolymerase that Forms an ATP-Hydrolyzing Complex at Microtubule Ends. Molecular Cell, 11(2), 445-457. https://doi.org/10.1016/S1097-2765(03)00049-2

Hutter, J. L., \& Bechhoefer, J. (1993). Calibration of atomic-force microscope tips. Review of Scientific Instruments, 64(7), 1868-1873. https://doi.org/10.1063/1.1143970

Inoué, S., \& Ritter, H. (1975). Dynamics of mitotic spindle organization and function. Society of General Physiologists Series, 30, 3-30.

Ivanov, A. I., Hopkins, A. M., Brown, G. T., Gerner-Smidt, K., Babbin, B. A., Parkos, C. A., \& Nusrat, A. (2008). Myosin II regulates the shape of three-dimensional intestinal epithelial cysts. Journal of Cell Science, 121(11), 1803-1814. https://doi.org/10.1242/jcs.015842

Jackson, S. P., \& Bartek, J. (2009). The DNA-damage response in human biology and disease. Nature, 461(7267), 1071-1078. https://doi.org/10.1038/nature08467

Jordan, M. A., Thrower, D., \& Wilson, L. (1992). Effects of vinblastine, podophyllotoxin and nocodazole on mitotic spindles. Implications for the role of microtubule dynamics in mitosis. Journal of Cell Science, 102 (Pt 3), 401-416.

Kalab, P., \& Heald, R. (2008). The RanGTP gradient - a GPS for the mitotic spindle. Journal of Cell Science, 121(Pt 10), 1577-1586. https://doi.org/10.1242/jcs.005959

Katoh, H., Hiramoto, K., \& Negishi, M. (2006). Activation of Rac1 by RhoG regulates cell migration. Journal of Cell Science, 119(Pt 1), 56-65. https://doi.org/10.1242/jcs.02720

Kaverina, I., \& Straube, A. (2011). Regulation of cell migration by dynamic microtubules. Seminars in Cell \& Developmental Biology, 22(9), 968-974. https://doi.org/10.1016/j.semcdb.2011.09.017

Kelly, A. E., Kranitz, H., Dötsch, V., \& Mullins, R. D. (2006). Actin binding to the central domain of WASP/Scar proteins plays a critical role in the activation of the Arp2/3 complex. The Journal of Biological Chemistry, 281(15), 10589-10597.

https://doi.org/10.1074/jbc.M507470200

Khodjakov, A., Cole, R. W., Oakley, B. R., \& Rieder, C. L. (2000). Centrosome-independent mitotic spindle formation in vertebrates. Current Biology, 10(2), 59-67.

https://doi.org/10.1016/S0960-9822(99)00276-6 
Kim, K., Lee, S.-A., \& Park, D. (2019). Emerging Roles of Ephexins in Physiology and Disease. Cells, 8(2). https://doi.org/10.3390/cells8020087

Kirschner, M., \& Mitchison, T. (1986). Beyond self-assembly: From microtubules to morphogenesis. Cell, 45(3), 329-342. https://doi.org/10.1016/0092-8674(86)90318-1

Knaus, U. G., \& Bokoch, G. M. (1998). The p21Rac/Cdc42-activated kinases (PAKs). The International Journal of Biochemistry \& Cell Biology, 30(8), 857-862. https://doi.org/10.1016/S1357-2725(98)00059-4

Konishi, H., Kikuchi, S., Ochiai, T., Ikoma, H., Kubota, T., Ichikawa, D., Fujiwara, H., Okamoto, K., Sakakura, C., Sonoyama, T., Kokuba, Y., Sasaki, H., Matsui, T., \& Otsuji, E. (2009). Latrunculin a has a strong anticancer effect in a peritoneal dissemination model of human gastric cancer in mice. Anticancer Research, 29(6), 2091-2097.

Kops, G. J. P. L., Weaver, B. A. A., \& Cleveland, D. W. (2005). On the road to cancer: Aneuploidy and the mitotic checkpoint. Nature Reviews. Cancer, 5(10), 773-785. https://doi.org/10.1038/nrc1714

Kosako, H., Yoshida, T., Matsumura, F., Ishizaki, T., Narumiya, S., \& Inagaki, M. (2000). Rhokinase/ROCK is involved in cytokinesis through the phosphorylation of myosin light chain and not ezrin/radixin/moesin proteins at the cleavage furrow. Oncogene, 19(52), 6059-6064. https://doi.org/10.1038/sj.onc. 1203987

Kozlowski, C., Srayko, M., \& Nedelec, F. (2007). Cortical microtubule contacts position the spindle in C. Elegans embryos. Cell, 129(3), 499-510.

https://doi.org/10.1016/j.cell.2007.03.027

Kronlage, C., Schäfer-Herte, M., Böning, D., Oberleithner, H., \& Fels, J. (2015). Feeling for Filaments: Quantification of the Cortical Actin Web in Live Vascular Endothelium. Biophysical Journal, 109(4), 687-698. https://doi.org/10.1016/j.bpj.2015.06.066

Kühn, S., \& Geyer, M. (2014). Formins as effector proteins of Rho GTPases. Small GTPases, 5, e29513. https://doi.org/10.4161/sgtp.29513

Kunda, P., \& Baum, B. (2009). The actin cytoskeleton in spindle assembly and positioning. Trends in Cell Biology, 19(4), 174-179. https://doi.org/10.1016/j.tcb.2009.01.006

Kunda, P., Pelling, A. E., Liu, T., \& Baum, B. (2008). Moesin controls cortical rigidity, cell rounding, and spindle morphogenesis during mitosis. Current Biology, 18(2), 91-101. https://doi.org/10.1016/j.cub.2007.12.051 
Kwon, M., Bagonis, M., Danuser, G., \& Pellman, D. (2015). Direct Microtubule-Binding by Myosin-10 Orients Centrosomes toward Retraction Fibers and Subcortical Actin Clouds. Developmental Cell, 34(3), 323-337. https://doi.org/10.1016/j.devcel.2015.06.013.

Laevsky, G., \& Knecht, D. A. (2003). Cross-linking of actin filaments by myosin II is a major contributor to cortical integrity and cell motility in restrictive environments. Journal of Cell Science, 116(Pt 18), 3761-3770. https://doi.org/10.1242/jcs.00684

Lancaster, O. M., \& Baum, B. (2014). Shaping up to divide: Coordinating actin and microtubule cytoskeletal remodelling during mitosis. Seminars in Cell \& Developmental Biology, 34, 109115. https://doi.org/10.1016/j.semcdb.2014.02.015

Lancaster, O. M., Le Berre, M., Dimitracopoulos, A., Bonazzi, D., Zlotek-Zlotkiewicz, E., Picone, R., Duke, T., Piel, M., \& Baum, B. (2013). Mitotic Rounding Alters Cell Geometry to Ensure Efficient Bipolar Spindle Formation. Developmental Cell, 25(3), 270-283. https://doi.org/10.1016/j.devcel.2013.03.014

Lane, J., Martin, T. A., Mansel, R. E., \& Jiang, W. G. (2008). The expression and prognostic value of the guanine nucleotide exchange factors (GEFs) Trio, Vav1 and TIAM-1 in human breast cancer. International Seminars in Surgical Oncology: ISSO, 5, 23.

https://doi.org/10.1186/1477-7800-5-23

Lara-Gonzalez, P., Westhorpe, F. G., \& Taylor, S. S. (2012). The Spindle Assembly Checkpoint. Current Biology, 22(22), R966-R980. https://doi.org/10.1016/j.cub.2012.10.006

Laskey, R. A., Fairman, M. P., \& Blow, J. J. (1989). S phase of the cell cycle. Science, 246(4930), 609-614. https://doi.org/10.1126/science.2683076

Lauffenburger, D. A., \& Horwitz, A. F. (1996). Cell Migration: A Physically Integrated Molecular Process. Cell, 84(3), 359-369. https://doi.org/10.1016/S0092-8674(00)81280-5

Lawson, C. D., \& Ridley, A. J. (2018). Rho GTPase signaling complexes in cell migration and invasion. The Journal of Cell Biology, 217(2), 447-457. https://doi.org/10.1083/jcb.201612069

Lázaro-Diéguez, F., Ispolatov, I., \& Müsch, A. (2015). Cell shape impacts on the positioning of the mitotic spindle with respect to the substratum. Molecular Biology of the Cell, 26(7), 12861295. https://doi.org/10.1091/mbc.E14-08-1330

Lecland, N., \& Lüders, J. (2014). The dynamics of microtubule minus ends in the human mitotic spindle. Nature Cell Biology, 16(8), 770-778. https://doi.org/10.1038/ncb2996 
Lee, J. C., Field, D. J., \& Lee, L. L. (1980). Effects of nocodazole on structures of calf brain tubulin. Biochemistry, 19(26), 6209-6215. https://doi.org/10.1021/bi00567a041

Lepage, C. C., Morden, C. R., Palmer, M. C. L., Nachtigal, M. W., \& McManus, K. J. (2019). Detecting Chromosome Instability in Cancer: Approaches to Resolve Cell-to-Cell Heterogeneity. Cancers, 11(2). https://doi.org/10.3390/cancers 11020226

Liskovykh, M., Goncharov, N. V., Petrov, N., Aksenova, V., Pegoraro, G., Ozbun, L. L., Reinhold, W. C., Varma, S., Dasso, M., Kumeiko, V., Masumoto, H., Earnshaw, W. C., Larionov, V., \& Kouprina, N. (2019). A novel assay to screen siRNA libraries identifies protein kinases required for chromosome transmission. Genome Research, 29(10), 1719-1732. https://doi.org/10.1101/gr.254276.119.

Lo, A. W.I., Sabatier, L., Fouladi, B., Pottier, G., Ricoul, M., \& Mumane, J. P. (2002). DNA Amplification by Breakage/Fusion/Bridge Cycles Initiated by Spontaneous Telomere Loss in a Human Cancer Cell Line. Neoplasia, 4(6), 531-538. https://doi.org/10.1038/sj.neo.7900267

Lolli, G., \& Johnson, L. N. (2005). CAK-Cyclin-Dependent Activating Kinase: A Key Kinase in Cell Cycle Control and a Target for Drugs? Cell Cycle, 4(4), 565-570.

https://doi.org/10.4161/cc.4.4.1607

Lopez, V., Barinova, N., Onishi, M., Pobiega, S., Pringle, J. R., Dubrana, K., \& Marcand, S. (2015). Cytokinesis breaks dicentric chromosomes preferentially at pericentromeric regions and telomere fusions. Genes \& Development, 29(3), 322-336.

https://doi.org/10.1101/gad.254664.114

Lu, Y., Chen, J., Xiao, M., Li, W., \& Miller, D. D. (2012). An overview of tubulin inhibitors that interact with the colchicine binding site. Pharmaceutical Research, 29(11), 2943-2971. https://doi.org/10.1007/s11095-012-0828-z

Luo, T., \& Robinson, D. N. (2010). Cooperative Interactions Between Myosin II and Actin CrossLinking Proteins To Actin Filaments. Biophysical Journal, 98(3), 18a. https://doi.org/10.1016/j.bpj.2009.12.112

Machicoane, M., Frutos, C. A. de, Fink, J., Rocancourt, M., Lombardi, Y., Garel, S., Piel, M., \& Echard, A. (2014). Slk-dependent activation of ERMs controls LGN-NuMA localization and spindle orientation. The Journal of Cell Biology, 205(6), 791-799.

https://doi.org/10.1083/jcb.201401049 
Maddox, A. S., \& Burridge, K. (2003). Rhoa is required for cortical retraction and rigidity during mitotic cell rounding. The Journal of Cell Biology, 160(2), 255-265.

https://doi.org/10.1083/jcb.200207130

Maier, M., Baldwin, C., Aoudjit, L., \& Takano, T. (2018). The Role of Trio, a Rho Guanine Nucleotide Exchange Factor, in Glomerular Podocytes. International Journal of Molecular Sciences, 19(2). https://doi.org/10.3390/ijms19020479

Malumbres, M., \& Barbacid, M. (2005). Mammalian cyclin-dependent kinases. Trends in Biochemical Sciences, 30(11), 630-641. https://doi.org/10.1016/j.tibs.2005.09.005

Maréchal, A., \& Zou, L. (2013). Dna Damage Sensing by the ATM and ATR Kinases. Cold Spring Harbor Perspectives in Biology, 5(9). https://doi.org/10.1101/cshperspect.a012716

Maresca, T. J., \& Salmon, E. D. (2010). Welcome to a new kind of tension: Translating kinetochore mechanics into a wait-anaphase signal. Journal of Cell Science, 123(Pt 6), 825835. https://doi.org/10.1242/jcs.064790

Martin, G. A., Bollag, G., McCormick, F., \& Abo, A. (1995). A novel serine kinase activated by rac1/CDC42Hs-dependent autophosphorylation is related to PAK65 and STE20. The EMBO Journal, 14(9), 1970-1978.

Martin, K., Reimann, A., Fritz, R. D., Ryu, H., Jeon, N. L., \& Pertz, O. (2016). Spatio-temporal co-ordination of RhoA, Rac1 and Cdc42 activation during prototypical edge protrusion and retraction dynamics. Scientific Reports, 6(1), 1-14. https://doi.org/10.1038/srep21901

Matthews, H. K., Delabre, U., Rohn, J. L., Guck, J., Kunda, P., \& Baum, B. (2012). Changes in Ect2 localization couple actomyosin-dependent cell shape changes to mitotic progression. Developmental Cell, 23(2), 371-383. https://doi.org/10.1016/j.devcel.2012.06.003

May, M., Schelle, I., Brakebusch, C., Rottner, K., \& Genth, H. (2014). Rac1-dependent recruitment of PAK2 to $\mathrm{G} 2$ phase centrosomes and their roles in the regulation of mitotic entry. Cell Cycle, 13(14), 2211-2221. https://doi.org/10.4161/cc.29279

Melak, M., Plessner, M., \& Grosse, R. (2017). Actin visualization at a glance. Journal of Cell Science, 130(3), 525-530. https://doi.org/10.1242/jcs.189068

Meunier, S., \& Vernos, I. (2016). Acentrosomal Microtubule Assembly in Mitosis: The Where, When, and How. Trends in Cell Biology, 26(2), 80-87.

https://doi.org/10.1016/j.tcb.2015.09.001 
Michaelis, L., \& Menten, M. L. (1913). Die Kinetik der Invertinwirkung. Biochemische Zeitschrift, 49, 333-369.

Michiels, F., Habets, G. G., Stam, J. C., van der Kammen, R. A., \& Collard, J. G. (1995). A role for Rac in Tiam1-induced membrane ruffling and invasion. Nature, 375(6529), 338-340. https://doi.org/10.1038/375338a0

Millar, F. R., Janes, S. M., \& Giangreco, A. (2017). Epithelial cell migration as a potential therapeutic target in early lung cancer. European Respiratory Review: An Official Journal of the European Respiratory Society, 26(143). https://doi.org/10.1183/16000617.0069-2016

Milunovic-Jevtic, A., Mooney, P., Sulerud, T., Bisht, J., \& Gatlin, J. C. (2016). Centrosomal Clustering Contributes to Chromosomal Instability and Cancer. Current Opinion in Biotechnology, 40, 113-118. https://doi.org/10.1016/j.copbio.2016.03.011

Mimori-Kiyosue, Y., Shiina, N., \& Tsukita, S. (2000). The dynamic behavior of the APC-binding protein EB1 on the distal ends of microtubules. Current Biology, 10(14), 865-868. https://doi.org/10.1016/S0960-9822(00)00600-X

Minard, M. E., Kim, L.-S., Price, J. E., \& Gallick, G. E. (2004). The role of the guanine nucleotide exchange factor Tiam1 in cellular migration, invasion, adhesion and tumor progression. Breast Cancer Research and Treatment, 84(1), 21-32. https://doi.org/10.1023/B:BREA.0000018421.31632.e6

Minhas, K. M., Singh, B., Jiang, W.-W., Sidransky, D., \& Califano, J. A. (2003). Spindle assembly checkpoint defects and chromosomal instability in head and neck squamous cell carcinoma. International Journal of Cancer, 107(1), 46-52. https://doi.org/10.1002/ijc.11341

Mitchison, T. J. (1992). Actin based motility on retraction fibers in mitotic PtK2 cells. Cell Motility and the Cytoskeleton, 22(2), 135-151. https://doi.org/10.1002/cm.970220207

Mitsushima, M., Aoki, K., Ebisuya, M., Matsumura, S., Yamamoto, T., Matsuda, M., Toyoshima, F., \& Nishida, E. (2010). Revolving movement of a dynamic cluster of actin filaments during mitosis. The Journal of Cell Biology, 191(3), 453-462. https://doi.org/10.1083/jcb.201007136

Morgan, D. O. (1997). Cyclin-dependent kinases: Engines, clocks, and microprocessors. Annual Review of Cell and Developmental Biology, 13, 261-291. https://doi.org/10.1146/annurev.cellbio.13.1.261 
Morgan, W. F., Corcoran, J., Hartmann, A., Kaplan, M. I., Limoli, C. L., \& Ponnaiya, B. (1998). DNA double-strand breaks, chromosomal rearrangements, and genomic instability. Mutation Research/Fundamental and Molecular Mechanisms of Mutagenesis, 404(1-2), 125-128. https://doi.org/10.1016/S0027-5107(98)00104-3

Morin, X., \& Bellaïche, Y. (2011). Mitotic spindle orientation in asymmetric and symmetric cell divisions during animal development. Developmental Cell, 21(1), 102-119.

https://doi.org/10.1016/j.devcel.2011.06.012

Murray, A. W., \& Hunt, T. (1993). The cell cycle: An introduction. Oxford University Press.

Nakajima, M., Kumada, K., Hatakeyama, K., Noda, T., Peters, J.-M., \& Hirota, T. (2007). The complete removal of cohesin from chromosome arms depends on separase. Journal of Cell Science, 120(Pt 23), 4188-4196. https://doi.org/10.1242/jcs.011528

Nakamura, F., Osborn, T. M., Hartemink, C. A., Hartwig, J. H., \& Stossel, T. P. (2007). Structural basis of filamin A functions. The Journal of Cell Biology, 179(5), 1011-1025. https://doi.org/10.1083/jcb.200707073

Nigg, E. A. (2001). Mitotic kinases as regulators of cell division and its checkpoints. Nature Reviews. Molecular Cell Biology, 2(1), 21-32. https://doi.org/10.1038/35048096

Nishimura, K., Johmura, Y., Deguchi, K., Jiang, Z., Uchida, K. S. K., Suzuki, N., Shimada, M., Chiba, Y., Hirota, T., Yoshimura, S. H., Kono, K., \& Nakanishi, M. (2019). Cdk1-mediated DIAPH1 phosphorylation maintains metaphase cortical tension and inactivates the spindle assembly checkpoint at anaphase. Nature Communications, 10(1), 1-12. https://doi.org/10.1038/s41467-019-08957-w

Nunez, J., Fellous, A., Francon, J., \& Lennon, A. M. (1979). Competitive inhibition of colchicine binding to tubulin by microtubule-associated proteins. Proceedings of the National Academy of Sciences of the United States of America, 76(1), 86-90.

https://doi.org/10.1073/pnas.76.1.86

Orr, B., \& Compton, D. A. (2013). A Double-Edged Sword: How Oncogenes and Tumor Suppressor Genes Can Contribute to Chromosomal Instability. Frontiers in Oncology, 3, 164. https://doi.org/10.3389/fonc.2013.00164 
Osaki, J. H., Espinha, G., Magalhaes, Y. T., \& Forti, F. L. (2016). Modulation of RhoA GTPase Activity Sensitizes Human Cervix Carcinoma Cells to $Y$-Radiation by Attenuating DNA Repair Pathways. Oxidative Medicine and Cellular Longevity, 2016, 6012642. https://doi.org/10.1155/2016/6012642

Padrick, S. B., \& Rosen, M. K. (2010). Physical mechanisms of signal integration by WASP family proteins. Annual Review of Biochemistry, 79, 707-735. https://doi.org/10.1146/annurev.biochem.77.060407.135452

Pan, F., Egile, C., Lipkin, T., \& Li, R. (2004). Arpc1/arc40 mediates the interaction of the actinrelated protein 2 and 3 complex with Wiskott-Aldrich syndrome protein family activators. The Journal of Biological Chemistry, 279(52), 54629-54636.

https://doi.org/10.1074/jbc.M402357200

Passerini, V., Ozeri-Galai, E., Pagter, M. S. d., Donnelly, N., Schmalbrock, S., Kloosterman, W. P., Kerem, B., \& Storchová, Z. (2016). The presence of extra chromosomes leads to genomic instability. Nature Communications, 7(1), 1-12.

https://doi.org/10.1038/ncomms10754

Paulamäki, J. (2017). The Effects of Inhibiting Mechanotransductive Cell Signaling Pathways in Human Bone Marrow Stromal Cells in 2D vs. 3d Culture.

http://dspace.cc.tut.fi/dpub/bitstream/123456789/25168/3/Paulam\%c3\%a4ki.pdf

Phuyal, S., \& Farhan, H. (2019). Multifaceted Rho GTPase Signaling at the Endomembranes. Frontiers in Cell and Developmental Biology, 7, 127. https://doi.org/10.3389/fcell.2019.00127

Pollard, T. D. (2016). Actin and Actin-Binding Proteins. Cold Spring Harbor Perspectives in Biology, 8(8). https://doi.org/10.1101/cshperspect.a018226

Prosser, S. L., \& Pelletier, L. (2017). Mitotic spindle assembly in animal cells: A fine balancing act. Nature Reviews. Molecular Cell Biology, 18(3), 187-201.

https://doi.org/10.1038/nrm.2016.162

Quintyne, N. J., Reing, J. E., Hoffelder, D. R., Gollin, S. M., \& Saunders, W. S. (2005). Spindle multipolarity is prevented by centrosomal clustering. Science, 307(5706), 127-129. https://doi.org/10.1126/science.1104905

Ramalingam, N., Zhao, H., Breitsprecher, D., Lappalainen, P., Faix, J., \& Schleicher, M. (2010). Phospholipids regulate localization and activity of mDia1 formin. European Journal of Cell Biology, 89(10), 723-732. https://doi.org/10.1016/j.ejcb.2010.06.001 
Ramanathan, S. P., Helenius, J., Stewart, M. P., Cattin, C. J., Hyman, A. A., \& Muller, D. J. (2015). Cdk1-dependent mitotic enrichment of cortical myosin II promotes cell rounding against confinement. Nature Cell Biology, 17(2), 148-159. https://doi.org/10.1038/ncb3098

Remmerbach, T. W., Wottawah, F., Dietrich, J., Lincoln, B., Wittekind, C., \& Guck, J. (2009). Oral cancer diagnosis by mechanical phenotyping. Cancer Research, 69(5), 1728-1732. https://doi.org/10.1158/0008-5472.CAN-08-4073

Rickman, K., \& Smogorzewska, A. (2019). Advances in understanding DNA processing and protection at stalled replication forks. The Journal of Cell Biology, 218(4), 1096-1107. https://doi.org/10.1083/jcb.201809012

Ridley, A. J. (2001). Rho GTPases and cell migration. Journal of Cell Science, 114(Pt 15), 2713-2722.

Ridley, A. J. (2015). Rho GTPase signalling in cell migration. Current Opinion in Cell Biology, 36, 103-112. https://doi.org/10.1016/j.ceb.2015.08.005

Rogers, S. L., Rogers, G. C., Sharp, D. J., \& Vale, R. D. (2002). Drosophila EB1 is important for proper assembly, dynamics, and positioning of the mitotic spindle. The Journal of Cell Biology, 158(5), 873-884. https://doi.org/10.1083/jcb.200202032

Rogers, S. L., Wiedemann, U., Häcker, U., Turck, C., \& Vale, R. D. (2004). Drosophila RhoGEF2 associates with microtubule plus ends in an EB1-dependent manner. Current Biology, 14(20), 1827-1833. https://doi.org/10.1016/j.cub.2004.09.078

Rojas, R. J., Yohe, M. E., Gershburg, S., Kawano, T., Kozasa, T., \& Sondek, J. (2007). Galphaq directly activates p63RhoGEF and Trio via a conserved extension of the Dbl homologyassociated pleckstrin homology domain. The Journal of Biological Chemistry, 282(40), 29201-29210. https://doi.org/10.1074/jbc.M703458200

Rooney, C., White, G., Nazgiewicz, A., Woodcock, S. A., Anderson, K. I., Ballestrem, C., \& Malliri, A. (2010). The Rac activator STEF (Tiam2) regulates cell migration by microtubulemediated focal adhesion disassembly. EMBO Reports, 11(4), 292-298.

https://doi.org/10.1038/embor.2010.10

Rosenblatt, J., Cramer, L. P., Baum, B., \& McGee, K. M. (2004). Myosin II-Dependent Cortical Movement Is Required for Centrosome Separation and Positioning during Mitotic Spindle Assembly. Cell, 117(3), 361-372. https://doi.org/10.1016/S0092-8674(04)00341-1 
Rouiller, I., Xu, X.-P., Amann, K. J., Egile, C., Nickell, S., Nicastro, D., Li, R., Pollard, T. D., Volkmann, N., \& Hanein, D. (2008). The structural basis of actin filament branching by the Arp2/3 complex. The Journal of Cell Biology, 180(5), 887-895.

https://doi.org/10.1083/jcb.200709092

Ryu, T. H., Kim, J.-H., \& Kim, J. K. (2016). Chromosomal Aberrations in Human Peripheral Blood Lymphocytes after Exposure to lonizing Radiation. Genome Integrity, 7, 5. https://doi.org/10.4103/2041-9414.197172

Sajesh, B. V., Lichtensztejn, Z., \& McManus, K. J. (2013). Sister chromatid cohesion defects are associated with chromosome instability in Hodgkin lymphoma cells. BMC Cancer, 13, 391. https://doi.org/10.1186/1471-2407-13-391

Sallmyr, A., \& Tomkinson, A. E. (2018). Repair of DNA double-strand breaks by mammalian alternative end-joining pathways. The Journal of Biological Chemistry, 293(27), 1053610546. https://doi.org/10.1074/jbc. TM117.000375

Sansregret, L., Vanhaesebroeck, B., \& Swanton, C. (2018). Determinants and clinical implications of chromosomal instability in cancer. Nature Reviews. Clinical Oncology, 15(3), 139-150. https://doi.org/10.1038/nrclinonc.2017.198

Schermuly, N. (2019). Regulation Of Spindle Orientation By A Mitotic Actin Pathway In Chromosomally Unstable Cancer Cells.

Schmidt, A., \& Hall, A. (2002). Guanine nucleotide exchange factors for Rho GTPases: Turning on the switch. Genes \& Development, 16(13), 1587-1609.

https://doi.org/10.1101/gad.1003302

Schmidt, S., \& Debant, A. (2014). Function and regulation of the Rho guanine nucleotide exchange factor Trio. Small GTPases, 5, e29769. https://doi.org/10.4161/sgtp.29769

Schoonen, P. M., Guerrero Llobet, S., \& van Vugt, M. A. T. M. (2019). Replication stress: Driver and therapeutic target in genomically instable cancers. Advances in Protein Chemistry and Structural Biology, 115, 157-201. https://doi.org/10.1016/bs.apcsb.2018.10.006

Schuyler, S. C., Wu, Y.-F., \& Kuan, V. J.-W. (2012). The Mad1-Mad2 balancing act-a damaged spindle checkpoint in chromosome instability and cancer. Journal of Cell Science, 125(Pt 18), 4197-4206. https://doi.org/10.1242/jcs.107037 
Seipel, K., Medley, Q. G., Kedersha, N. L., Zhang, X. A., O’Brien, S. P., Serra-Pages, C., Hemler, M. E., \& Streuli, M. (1999). Trio amino-terminal guanine nucleotide exchange factor domain expression promotes actin cytoskeleton reorganization, cell migration and anchorageindependent cell growth. Journal of Cell Science, 112 (Pt 12), 1825-1834.

Sheltzer, J. M. (2013). A transcriptional and metabolic signature of primary aneuploidy is present in chromosomally unstable cancer cells and informs clinical prognosis. Cancer Research, 73(21), 6401-6412. https://doi.org/10.1158/0008-5472.CAN-13-0749.

Silkworth, W. T., \& Cimini, D. (2012). Transient defects of mitotic spindle geometry and chromosome segregation errors. Cell Division, 7(1), 19.

https://doi.org/10.1186/1747-1028-7-19

Simone, A. de, Nédélec, F., \& Gönczy, P. (2016). Dynein Transmits Polarized Actomyosin Cortical Flows to Promote Centrosome Separation. Cell Reports, 14(9), 2250-2262. https://doi.org/10.1016/j.celrep.2016.01.077

Skibbens, R. V. (2009). Establishment of Sister Chromatid Cohesion Minireview. Current Biology: CB, 19(24), R1126-32. https://doi.org/10.1016/j.cub.2009.10.067

Sonoshita, M., Itatani, Y., Kakizaki, F., Sakimura, K., Terashima, T., Katsuyama, Y., Sakai, Y., \& Taketo, M. M. (2015). Promotion of colorectal cancer invasion and metastasis through activation of NOTCH-DAB1-ABL-RHOGEF protein TRIO. Cancer Discovery, 5(2), 198-211. https://doi.org/10.1158/2159-8290.CD-14-0595

Steffen, A., Ladwein, M., Dimchev, G. A., Hein, A., Schwenkmezger, L., Arens, S., Ladwein, K. I., Margit Holleboom, J., Schur, F., Victor Small, J., Schwarz, J., Gerhard, R., Faix, J., Stradal, T. E. B., Brakebusch, C., \& Rottner, K. (2013). Rac function is crucial for cell migration but is not required for spreading and focal adhesion formation. Journal of Cell Science, 126(Pt 20), 4572-4588. https://doi.org/10.1242/jcs.118232

Stepanenko, A. A., \& Dmitrenko, V. V. (2015). Hek293 in cell biology and cancer research: Phenotype, karyotype, tumorigenicity, and stress-induced genome-phenotype evolution. Gene, 569(2), 182-190. https://doi.org/10.1016/j.gene.2015.05.065

Stewart, M. P., Helenius, J., Toyoda, Y., Ramanathan, S. P., Muller, D. J., \& Hyman, A. A. (2011). Hydrostatic pressure and the actomyosin cortex drive mitotic cell rounding. Nature, 469(7329), 226-230. https://doi.org/10.1038/nature09642 
Stypula-Cyrus, Y., Mutyal, N. N., Dela Cruz, M., Kunte, D. P., Radosevich, A. J., Wali, R., Roy, H. K., \& Backman, V. (2014). End-binding protein 1 (EB1) up-regulation is an early event in colorectal carcinogenesis. FEBS Letters, 588(5), 829-835.

https://doi.org/10.1016/j.febslet.2014.01.046

Sugihara, Y., Taniguchi, H., Kushima, R., Tsuda, H., Kubota, D., Ichikawa, H., Sakamoto, K., Nakamura, Y., Tomonaga, T., Fujita, S., \& Kondo, T. (2012). Proteomic-based identification of the APC-binding protein EB1 as a candidate of novel tissue biomarker and therapeutic target for colorectal cancer. Journal of Proteomics, 75(17), 5342-5355. https://doi.org/10.1016/j.jprot.2012.06.013

Suryadinata, R., Sadowski, M., \& Sarcevic, B. (2010). Control of cell cycle progression by phosphorylation of cyclin-dependent kinase (CDK) substrates. Bioscience Reports, 30(4), 243-255. https://doi.org/10.1042/BSR20090171

Swaminathan, V., Mythreye, K., O’Brien, E. T., Berchuck, A., Blobe, G. C., \& Superfine, R. (2011). Mechanical stiffness grades metastatic potential in patient tumor cells and in cancer cell lines. Cancer Research, 71(15), 5075-5080. https://doi.org/10.1158/0008-5472.CAN-110247

Tanenbaum, M. E., \& Medema, R. H. (2010). Mechanisms of centrosome separation and bipolar spindle assembly. Developmental Cell, 19(6), 797-806.

https://doi.org/10.1016/j.devcel.2010.11.011

Théry, M., \& Bornens, M. (2006). Cell shape and cell division. Current Opinion in Cell Biology, 18(6), 648-657. https://doi.org/10.1016/j.ceb.2006.10.001

Théry, M., \& Bornens, M. (2008). Get round and stiff for mitosis. HFSP Journal, 2(2), 65-71. https://doi.org/10.2976/1.2895661

Théry, M., Racine, V., Pépin, A., Piel, M., Chen, Y., Sibarita, J.-B., \& Bornens, M. (2005). The extracellular matrix guides the orientation of the cell division axis. Nature Cell Biology, 7(10), 947-953. https://doi.org/10.1038/ncb1307

Thompson, S. L., Bakhoum, S. F., \& Compton, D. A. (2010). Mechanisms of chromosomal instability. Current Biology: CB, 20(6), R285-95. https://doi.org/10.1016/j.cub.2010.01.034

Tirnauer, J. S., \& Bierer, B. E. (2000). Eb1 proteins regulate microtubule dynamics, cell polarity, and chromosome stability. The Journal of Cell Biology, 149(4), 761-766.

https://doi.org/10.1083/jcb.149.4.761 
Tojkander, S., Gateva, G., \& Lappalainen, P. (2012). Actin stress fibers-assembly, dynamics and biological roles. Journal of Cell Science, 125(Pt 8), 1855-1864.

https://doi.org/10.1242/jcs.098087

Toledo, M. de, Anguille, C., Roger, L., Roux, P., \& Gadea, G. (2012). Cooperative anti-invasive effect of Cdc42/Rac1 activation and ROCK inhibition in SW620 colorectal cancer cells with elevated blebbing activity. PloS One, 7(11), e48344.

https://doi.org/10.1371/journal.pone.0048344

Tolias, K. F., Hartwig, J. H., Ishihara, H., Shibasaki, Y., Cantley, L. C., \& Carpenter, C. L. (2000). Type la phosphatidylinositol-4-phosphate 5-kinase mediates Rac-dependent actin assembly. Current Biology, 10(3), 153-156. https://doi.org/10.1016/S0960-9822(00)00315-8

Toyoda, Y., Cattin, C. J., Stewart, M. P., Poser, I., Theis, M., Kurzchalia, T. V., Buchholz, F., Hyman, A. A., \& Müller, D. J. (2017). Genome-scale single-cell mechanical phenotyping reveals disease-related genes involved in mitotic rounding. Nature Communications, 8(1), 111. https://doi.org/10.1038/s41467-017-01147-6

Tubbs, A., \& Nussenzweig, A. (2017). Endogenous DNA Damage as a Source of Genomic Instability in Cancer. Cell, 168(4), 644-656. https://doi.org/10.1016/j.cell.2017.01.002

van Gent, D. C., Hoeijmakers, J. H., \& Kanaar, R. (2001). Chromosomal stability and the DNA double-stranded break connection. Nature Reviews. Genetics, 2(3), 196-206.

https://doi.org/10.1038/35056049

van Haren, J., Boudeau, J., Schmidt, S., Basu, S., Liu, Z., Lammers, D., Demmers, J., Benhari, J., Grosveld, F., Debant, A., \& Galjart, N. (2014). Dynamic microtubules catalyze formation of navigator-TRIO complexes to regulate neurite extension. Current Biology: $C B$, 24(15), 1778-1785. https://doi.org/10.1016/j.cub.2014.06.037

van Heesbeen, R. G. H. P., Raaijmakers, J. A., Tanenbaum, M. E., \& Medema, R. H. (2013). Nuclear envelope-associated dynein cooperates with Eg5 to drive prophase centrosome separation. Communicative \& Integrative Biology, 6(3), e23841. https://doi.org/10.4161/cib.23841

van Leeuwen, F. N., van der Kammen, R. A., Habets, G. G., \& Collard, J. G. (1995). Oncogenic activity of Tiam1 and Rac1 in NIH3T3 cells. Oncogene, 11(11), 2215-2221. 
van Rijssel, J., Hoogenboezem, M., Wester, L., Hordijk, P. L., \& van Buul, J. D. (2012). The Nterminal DH-PH domain of Trio induces cell spreading and migration by regulating lamellipodia dynamics in a Rac1-dependent fashion. PloS One, 7(1), e29912. https://doi.org/10.1371/journal.pone.0029912

van Rijssel, J., Timmerman, I., van Alphen, F. P. J., Hoogenboezem, M., Korchynskyi, O., Geerts, D., Geissler, J., Reedquist, K. A., Niessen, H. W. M., \& van Buul, J. D. (2013). The Rho-GEF Trio regulates a novel pro-inflammatory pathway through the transcription factor Ets2. Biology Open, 2(6), 569-579. https://doi.org/10.1242/bio.20134382

van Rijssel, J., \& van Buul, J. D. (2012). The many faces of the guanine-nucleotide exchange factor trio. Cell Adhesion \& Migration, 6(6), 482-487. https://doi.org/10.4161/cam.21418

Vaqué, J. P., Dorsam, R. T., Feng, X., Iglesias-Bartolome, R., Forsthoefel, D. J., Chen, Q., Debant, A., Seeger, M. A., Ksander, B. R., Teramoto, H., \& Gutkind, J. S. (2013). A genomewide RNAi screen reveals a Trio-regulated Rho GTPase circuitry transducing mitogenic signals initiated by G protein-coupled receptors. Molecular Cell, 49(1), 94-108.

https://doi.org/10.1016/j.molcel.2012.10.018

Vasquez, R. J., Howell, B., Yvon, A. M., Wadsworth, P., \& Cassimeris, L. (1997). Nanomolar concentrations of nocodazole alter microtubule dynamic instability in vivo and in vitro.

Molecular Biology of the Cell, 8(6), 973-985. https://doi.org/10.1091/mbc.8.6.973

Vaughan, K. T. (2005). Tip maker and TIP marker; EB1 as a master controller of microtubule plus ends. The Journal of Cell Biology, 171(2), 197-200.

https://doi.org/10.1083/jcb.200509150

Walczak, C. E., Cai, S., \& Khodjakov, A. (2010). Mechanisms of chromosome behaviour during mitosis. Nature Reviews Molecular Cell Biology, 11(2), 91-102.

https://doi.org/10.1038/nrm2832

Wang, B., Fang, J., Qu, L., Cao, Z., Zhou, J., \& Deng, B. (2015). Upregulated TRIO expression correlates with a malignant phenotype in human hepatocellular carcinoma. Tumour Biology: The Journal of the International Society for Oncodevelopmental Biology and Medicine, 36(9), 6901-6908. https://doi.org/10.1007/s13277-015-3377-3

Watanabe, T., Wang, S., \& Kaibuchi, K. (2015). IQGAPs as Key Regulators of Actincytoskeleton Dynamics. Cell Structure and Function, 40(2), 69-77. https://doi.org/10.1247/csf.15003 
Waterman-Storer, C. M., Worthylake, R. A., Liu, B. P., Burridge, K., \& Salmon, E. D. (1999). Microtubule growth activates Rac1 to promote lamellipodial protrusion in fibroblasts. Nature Cell Biology, 1(1), 45-50. https://doi.org/10.1038/9018

Wear, M. A., Yamashita, A., Kim, K., Maéda, Y., \& Cooper, J. A. (2003). How Capping Protein Binds the Barbed End of the Actin Filament. Current Biology, 13(17), 1531-1537. https://doi.org/10.1016/S0960-9822(03)00559-1

Wen, Y., Eng, C. H., Schmoranzer, J., Cabrera-Poch, N., Morris, E. J. S., Chen, M., Wallar, B. J., Alberts, A. S., \& Gundersen, G. G. (2004). Eb1 and APC bind to mDia to stabilize microtubules downstream of Rho and promote cell migration. Nature Cell Biology, 6(9), 820-830. https://doi.org/10.1038/ncb1160

Whalley, H. J., Porter, A. P., Diamantopoulou, Z., White, G. R. M., Castañeda-Saucedo, E., \& Malliri, A. (2015). Cdk1 phosphorylates the Rac activator Tiam1 to activate centrosomal Pak and promote mitotic spindle formation. Nature Communications, 6, 7437.

https://doi.org/10.1038/ncomms8437

Wittmann, T., Hyman, A., \& Desai, A. (2001). The spindle: A dynamic assembly of microtubules and motors. Nature Cell Biology, 3(1), E28-34. https://doi.org/10.1038/35050669

Wittmann, T., Bokoch, G. M., \& Waterman-Storer, C. M. (2003). Regulation of leading edge microtubule and actin dynamics downstream of Rac1. The Journal of Cell Biology, 161(5), 845-851. https://doi.org/10.1083/jcb.200303082

Xia, S., Lim, Y. B., Zhang, Z., Wang, Y., Zhang, S., Lim, C. T., Yim, E. K.F., \& Kanchanawong, P. (2019). Nanoscale Architecture of the Cortical Actin Cytoskeleton in Embryonic Stem Cells. Cell Reports, 28(5), 1251-1267.e7. https://doi.org/10.1016/j.celrep.2019.06.089

Yamaguchi, H., \& Condeelis, J. (2007). Regulation of the actin cytoskeleton in cancer cell migration and invasion. Biochimica Et Biophysica Acta (BBA) - Molecular Cell Research, 1773(5), 642-652. https://doi.org/10.1016/j.bbamcr.2006.07.001

Yang, C.-P. H., \& Horwitz, S. B. (2017). Taxol®: The First Microtubule Stabilizing Agent. International Journal of Molecular Sciences, 18(8). https://doi.org/10.3390/ijms18081733 
Yokoyama, H., Gruss, O. J., Rybina, S., Caudron, M., Schelder, M., Wilm, M., Mattaj, I. W., \& Karsenti, E. (2008). Cdk11 is a RanGTP-dependent microtubule stabilization factor that regulates spindle assembly rate. The Journal of Cell Biology, 180(5), 867-875. https://doi.org/10.1083/jcb.200706189

Yoshizaki, H., Ohba, Y., Kurokawa, K., Itoh, R. E., Nakamura, T., Mochizuki, N., Nagashima, K., \& Matsuda, M. (2003). Activity of Rho-family GTPases during cell division as visualized with FRET-based probes. The Journal of Cell Biology, 162(2), 223-232.

https://doi.org/10.1083/jcb.200212049

Yoshizaki, H., Ohba, Y., Parrini, M.-C., Dulyaninova, N. G., Bresnick, A. R., Mochizuki, N., \& Matsuda, M. (2004). Cell type-specific regulation of RhoA activity during cytokinesis. The Journal of Biological Chemistry, 279(43), 44756-44762.

https://doi.org/10.1074/jbc.M402292200

Zheng, Z., Zhu, H., Wan, Q., Liu, J., Xiao, Z., Siderovski, D. P., \& Du, Q. (2010). Lgn regulates mitotic spindle orientation during epithelial morphogenesis. The Journal of Cell Biology, 189(2), 275-288. https://doi.org/10.1083/jcb.200910021

Zhou, B. B., \& Elledge, S. J. (2000). The DNA damage response: Putting checkpoints in perspective. Nature, 408(6811), 433-439. https://doi.org/10.1038/35044005 


\section{Acknowledgement - Danksagung}

Ich danke allen, die mich während der letzten Jahre sowohl beim Gelingen dieser Arbeit, aber auch mich als Menschen so sehr unterstützt haben.

Zunächst möchte ich mich herzlich bei Prof. Dr. Lutz Walter bedanken, dass er die Betreuung und Erstbegutachtung meiner Dissertation in der Schlussphase so kurzfristig übernommen hat.

Ebenso bedanke ich mich bei Prof. Dr. Holger Bastians für die Bereitstellung des Projektes sowie für die Begleitung der Labortätigkeiten. Bei den Mitgliedern meines Thesis Advisory Commitees Dr. Melina Schuh, Dr. Julia Groß und Prof. Dr. Matthias Dobbelstein möchte ich mich für die gute Betreuung und die hilfreichen Ideen ebenfalls herzlich bedanken. Zudem gilt mein Dank Prof. Dr. Heike Krebber für die Bereitstellung von Laborräumen und Arbeitsausrüstung.

Besonders möchte ich mich auch beim GGNB und insbesondere bei Kirsten Pöhlker bedanken: Ihr standet mir besonders in schwierigen Situationen immer mit Rat und Tat, sowie mit viel Empathie und Unterstützung zur Seite.

Weiterhin bedanke ich mich herzlich bei Prof. Dr. Andreas Janshoff und Dr. Bastian Rouven Brückner für die sehr interessante und abwechslungsreiche Kollaboration. Die Zusammenarbeit war nicht nur lehrreich, sondern hat mir auch viel Spaß bereitet.

Besonders möchte ich mich auch bei allen Mitgliedern der Arbeitsgruppe bedanken, mit denen ich nicht nur hervorragend Ideen austauschen konnte, sondern mit denen ich auch viele unterhaltsame Momente erleben durfte. Vielen Dank an Lena für die Unterstützung in vielen Situationen, und für die vielen lustigen Momente mit euch, Yu-Chih und Nico. Insbesondere möchte ich mich auch bei Nadine S., Karo, Ann-Kathrin, Katharina und Nadine Ü. bedanken, und zwar nicht nur für die zielführenden fachlichen Diskussionen, sondern vor allem für die gemeinsame und oftmals sehr unterhaltsame Zeit, die wir auch außerhalb des Labors hatten.

Mein größter Dank gilt jedoch meiner Familie, meinen Eltern Maria und Wilfried, meinen Brüdern Jan und Vincent und ganz besonders meinem Mann Pascal. Ihr wart und seid immer für mich da, unterstützt mich, glaubt an mich und schenkt mir so viel Liebe jeden Tag. Ich danke euch dafür von ganzem Herzen, denn nur mit euch war ein Gelingen dieser Arbeit möglich. 


\section{Curriculum Vitae}

\section{Personal Details}

$\begin{array}{ll}\text { Name } & \text { Elina Glaubke } \\ \text { Date and place of birth } & 27^{\text {th }} \text { August 1990, Osnabrück, Germany } \\ \text { Nationality } & \text { German }\end{array}$

\section{Education}

$06 / 2016-11 / 2019$

PhD Program "Molecular Biology of Cells"

- University Medical Center Göttingen, Germany

- Institute for Molecular Oncology

$10 / 2013-05 / 2016$

Master's Program "Biological Science"

- University of Osnabrück, Germany

- Field of study: Cell and Molecular Biology

- Program Degree: Master of Science

$10 / 2013-05 / 2016$

Master's Program "Biology" and "Chemistry"

- University of Osnabrück, Germany

- Program Degree: Master of Education

$10 / 2015-05 / 2016$

Master Thesis

- University Medical Center Hamburg-Eppendorf

- Institute for Biochemistry and Signal Transduction (PD Dr. Windhorst)

- Thesis Title: "Analysis of inositol 1,4,5-trisphosphate 3-kinase $B$ function in actin dynamics of human lymphocytes"

$03 / 2015-07 / 2015$

USA Internship

- University of Massachusetts Medical School, MA, Worcester

- Department of Biochemistry and Molecular Pharmacology (Prof. Mary Munson) and Department of Molecular, Cell and Cancer Biology (Prof. Peter Newburger) 
- Project Title: "Analysis of the Sec1/Munc18 protein VPS45 and neutropenia causing VPS45 mutations in HL-60 cells and in yeast"

$10 / 2011-09 / 2013$

Dual Bachelor's Program "Biology" and "Chemistry"

- University of Osnabrück, Germany

- Program Degree: Bachelor of Science

$04 / 2013-09 / 2013$

Bachelor Thesis

- University of Osnabrück, Germany

- Department of Biochemistry (Prof. Ungermann)

- Thesis Title: "Untersuchung des endolysosomalen HOPSTethering-Komplexes anhand der Isoformen von Vps39 in menschlichen Zellen“

10/2010 - 09/2011 Bachelor's Program “Molecular Life Science”

- University of Lübeck, Germany

08/2003-06/2010 High School

- Angela Gymnasium Osnabrück, Germany

- Graduation: Abitur

\section{Publications}

Glaubke, E., \& Bastians, H. (2018). A Cell-Based Assay for Mitotic Spindle Orientation. Methods in Molecular Biology (Clifton, N.J.), 1787, 67-75. https://doi.org/10.1007/978-1-49397847-2_5

Bäder, S., Glaubke, E., Grüb, S., Muhs, S., Wellbrock, J., Nalaskowski, M., Lange, T., \& Windhorst, S. (2018). Effect of the actin- and calcium-regulating activities of ITPKB on the metastatic potential of lung cancer cells. The Biochemical Journal, 475(12), 2057-2071. https://doi.org/10.1042/BCJ20180238

Lachmann, J., Glaubke, E., Moore, P. S., \& Ungermann, C. (2014). The Vps39-like TRAP1 is an effector of Rab5 and likely the missing Vps3 subunit of human CORVET. Cellular Logistics, 4(4), e970840. https://doi.org/10.4161/21592780.2014.970840 